\title{
On the Improvement of the Indirect Quantification of Methane Emissions: A Stationary Single Sensor Approach
}

\author{
Robert Scott Heltzel \\ West Virginia University, rheltzel@mix.wvu.edu
}

Follow this and additional works at: https://researchrepository.wvu.edu/etd

Part of the Energy Systems Commons, and the Other Mechanical Engineering Commons

\section{Recommended Citation}

Heltzel, Robert Scott, "On the Improvement of the Indirect Quantification of Methane Emissions: A Stationary Single Sensor Approach" (2021). Graduate Theses, Dissertations, and Problem Reports. 8329. https://researchrepository.wvu.edu/etd/8329

This Dissertation is protected by copyright and/or related rights. It has been brought to you by the The Research Repository @ WVU with permission from the rights-holder(s). You are free to use this Dissertation in any way that is permitted by the copyright and related rights legislation that applies to your use. For other uses you must obtain permission from the rights-holder(s) directly, unless additional rights are indicated by a Creative Commons license in the record and/ or on the work itself. This Dissertation has been accepted for inclusion in WVU Graduate Theses, Dissertations, and Problem Reports collection by an authorized administrator of The Research Repository @ WVU.

For more information, please contact researchrepository@mail.wvu.edu. 


\title{
On the Improvement of the Indirect Quantification of Methane Emissions: A Stationary Single Sensor Approach
}

\author{
Robert S. Heltzel \\ Dissertation submitted to the \\ Benjamin M. Statler College of Engineering and Mineral Resources \\ at West Virginia University \\ in partial fulfillment of the requirements for the degree of \\ Doctor of Philosophy \\ in \\ Mechanical Engineering \\ Derek Johnson, PhD, Chair \\ Gil Bohrer, PhD \\ Cosmin Dumitrescu, $\mathrm{PhD}$ \\ Andrew Nix, $\mathrm{PhD}$ \\ Arvind Thiruvengadam, $\mathrm{PhD}$ \\ Department of Mechanical and Aerospace Engineering \\ Morgantown, West Virginia \\ 2021
}

Keyword: Methane emissions, Indirect quantification, OTM 33A, Eddy covariance, Natural gas

Copyright 2021 Robert Heltzel 


\title{
Abstract \\ On the Improvement of the Indirect Quantification of Methane Emissions: A Stationary Single Sensor Approach
}

\begin{abstract}
Robert S. Heltzel
There is a need for improved indirect quantification of methane emissions, particularly from the natural gas industry. Natural gas production continues to increase along with the number of wells and production sites. These production sites have the potential to contribute significant levels of methane emissions. Methane is a potent greenhouse gas and the primary component of natural gas. A more complete understanding of emissions through improved quantification will lead to enhanced mitigation. Emissions have historically been quantified with both direct and indirect methods, with varying results. Emissions have been shown to be both temporally and spatially variable. These facts have led government agencies to fund programs that are focused on improving the quantification methods used. The National Science Foundation (NSF) recently awarded a grant to West Virginia University (WVU) that focused on the "inter-comparison and advancement" of direct and indirect methane emissions measurements. The primary objectives were to directly quantify emissions, develop and deploy a data acquisition system to record variables required for indirect measurements, and employ machine learning to combine current methods with the goal of reducing indirect quantification uncertainty.
\end{abstract}

To accomplish these objectives, two previously utilized techniques for quantifying methane fluxes were analyzed. OTM 33A (OTM) and eddy covariance (EC) are two unique measurement techniques typically used for different applications but requiring similar instrumentation. However, both techniques have high uncertainties (30-70\%) associated with their estimates. Methods were used to estimate minimal attainable uncertainty, which was determined to be $\pm 17 \%$ for OTM and $\pm 42 \%$ for EC. This lends credence to the hypothesis that indirect measurements from stationary single sensors will never obtain the accuracy of direct quantification estimates $(\sim 10 \%)$. Here, methods of point source estimation were enhanced through modification and combination with machine learning. The OTM method was enhanced by optimizing its governing parameters utilizing a Taguchi design array. This resulted in an average reduction of $22 \%$ in the root mean squared error (RMSE) and 30\% in the standard deviation of estimates across a series of controlled release experiments. EC footprint functions previously used to determine point source emissions were evaluated but produced results that tended to significantly underestimate emissions. Combining traditional method results with machine learning could improve indirect quantification. To test this hypothesis, a mobile eddy covariance tower (MECT) was developed with the ability to measure variables necessary for both techniques. The MECT was deployed to measure a series of controlled releases for testing and verification. Using this dataset, two machine learning techniques, a random forest (RF), and an artificial neural network $(\mathrm{NN})$, were used with results from the traditional methods. Models were enhanced with feature reduction and hyper-parameter tuning. Final RF and NN models produced lower RMSEs across controlled release datasets. The RF and NN improved the RMSE of the full test set, by $69 \%$ and $44 \%$ respectively, compared to Taguchi OTM estimates. These models were then utilized to predict emissions from an active production site, over the course of one year. The site emissions were compared to a direct quantification method used for quarterly audits. The NN and RF RMSEs were $49 \%$ and $9 \%$ lower on average than OTM estimates when compared to direct quantification audits.

The results of this research were two machine learning methods which utilized traditional quantification techniques to improve indirect estimates from a single stationary sensor. These methods could be further enhanced with a larger training test set and more verification data. Deploying a MECT at other active sites and utilizing these methods could continue to improve emissions estimates and provide more temporal information on the nature of methane emissions from the natural gas production sector. 


\section{Acknowledgements}

I would first and foremost like to thank Boss, my advisor, friend, and mentor: Dr. Derek Johnson. Without your support, guidance, and understanding none of this work would have been possible. There have been countless times when I did not think that I would ever complete, before realizing that excuses do not equal performance. Someday I hope to accurately quantify all that you have done for me throughout this process.

I would like to thank my committee members, Drs. Gil Bohrer, Cosmin Dumitrescu, Andrew Nix, and Arvind Thiruvengadam for taking the time and effort to assist in guiding me throughout this project. Without the wisdom of those with more experience than ourselves, little would be achieved.

Recognition is given to the National Science Foundation for funding the work performed here.

I would like to extend a special thanks to Dr. Mahdi Darzi. His dedication to all the right things in life is a continuous inspiration. His thoughtfulness, gratefulness, and work ethic are states of mind that I try to mimic every day.

I would also like to shout out the Master's students who assisted me in conducting my research. Amber Barr, Brian Cappelini, Diego Dranuta, and Dakota Oliver, all assisted me with my work. I appreciated our teams' "drop-everything-to-help" mindset in support of one another. I am grateful that I could count on the team to assist when needed. The friendships made the work feel a little bit less like work. Plus, everyone needs a TSY in their life, and I realize how rare it is to find one in the wild.

My brother, Brad, has done more for me than anyone over the past two years. It is nice to be able to talk to someone who also understands that "We're kids, we're not men." I appreciate his constant support and insight about life, school, and everything I pursue. I would also like to thank my Dad for his support throughout my endeavors. I am also indebted to the rest of my friends and family for everything they do for me.

I would like to also to take time to remember my Mom, Cindi Ann Scott. Everything I do in life is aided by your loving memory. Some hearts and thoughts do in fact not fade, fade away. 


\section{Table of Contents}

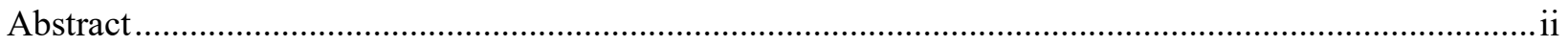

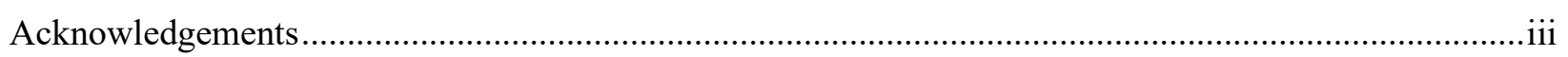

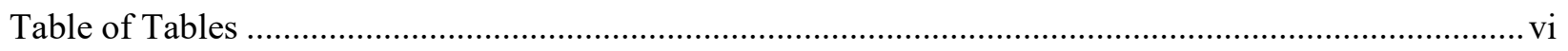

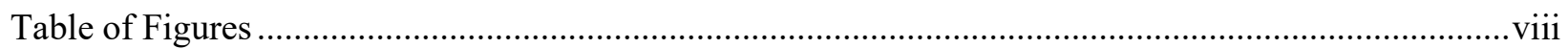

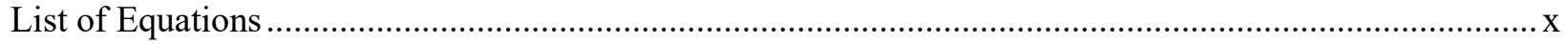

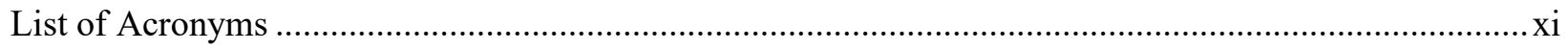

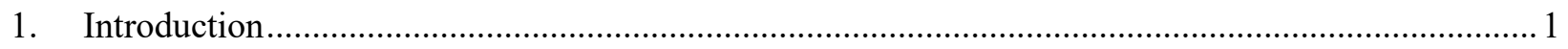

2. Literature Review/Need for Research.................................................................................. 4

2.1 Methane Emissions and the Natural Gas Industry .................................................................. 4

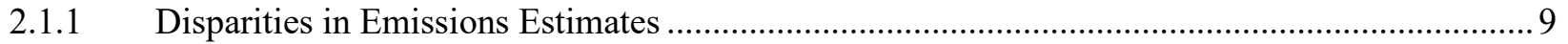

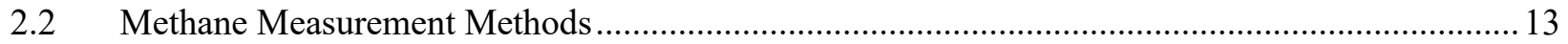

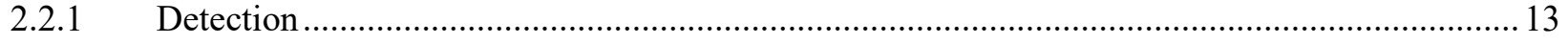

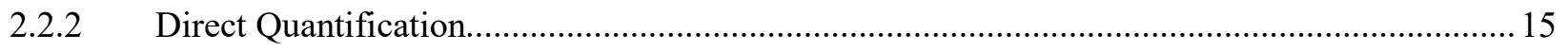

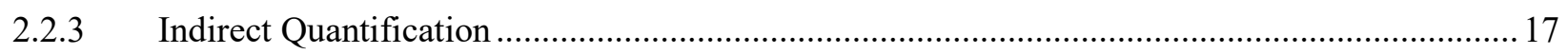

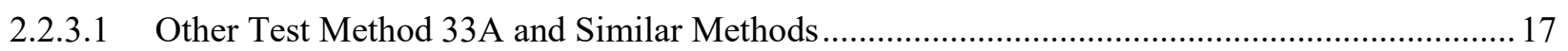

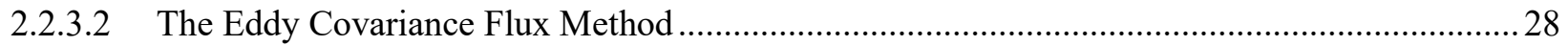

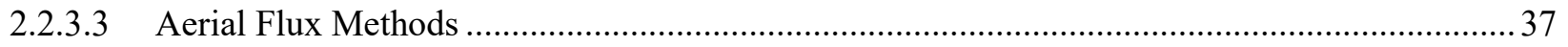

2.2.3.4 Latest Advances in Methane Quantification and Leak Detection............................................ 39

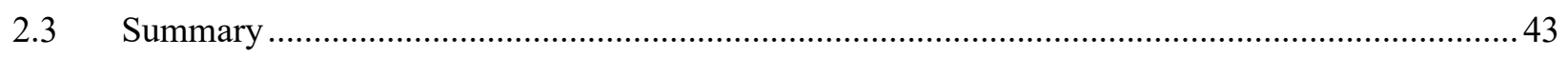

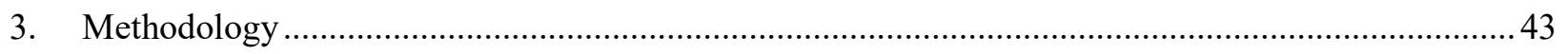

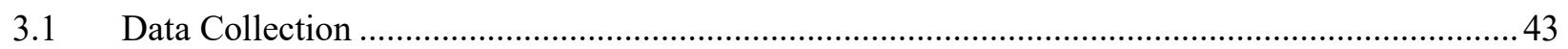

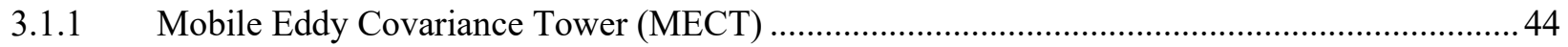

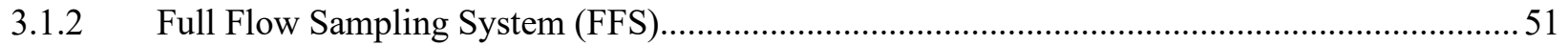

3.1.3 Marcellus Shale Energy and Environment Laboratory (MSEEL) Production Site.................... 54

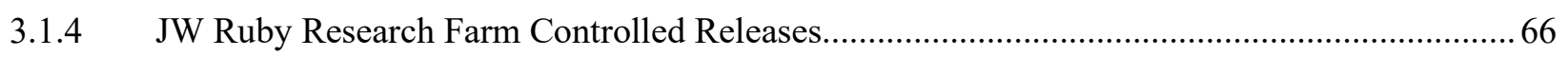

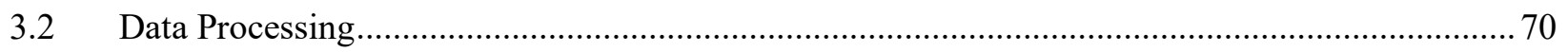

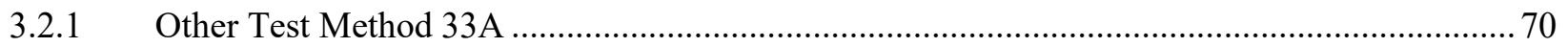

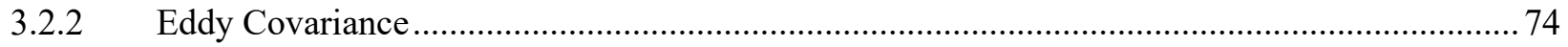

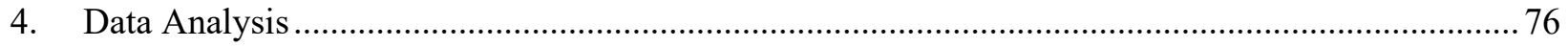

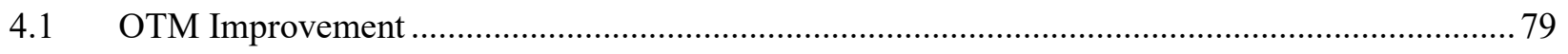

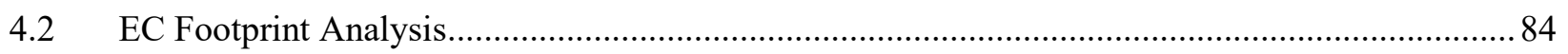

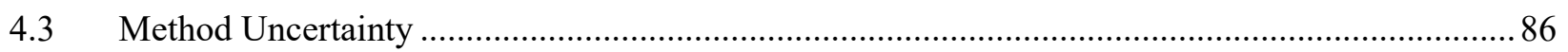

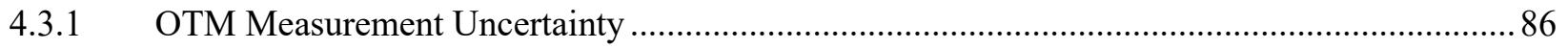

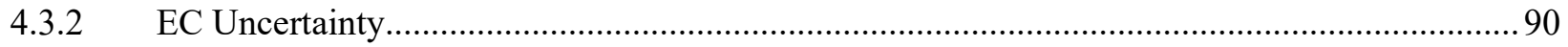

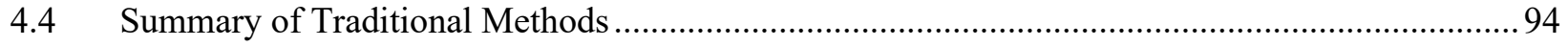




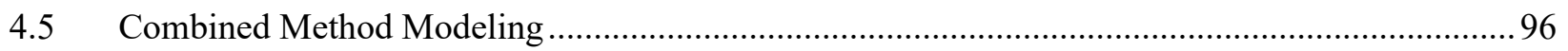

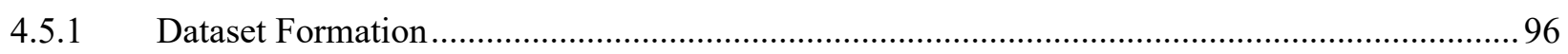

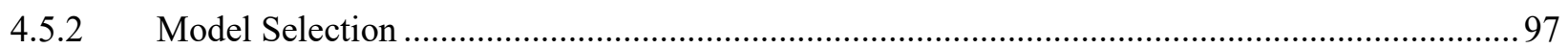

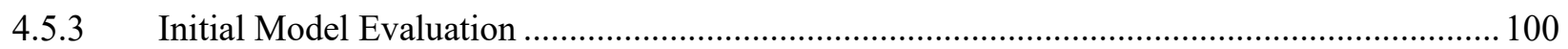

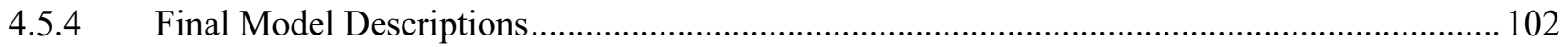

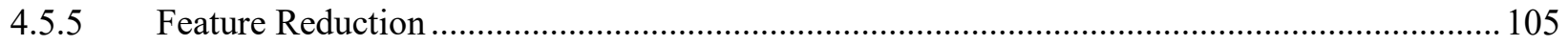

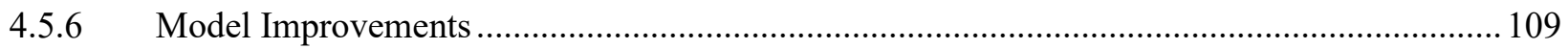

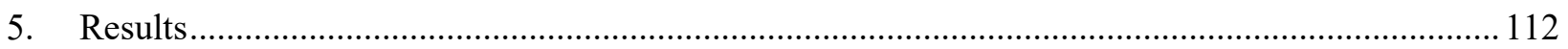

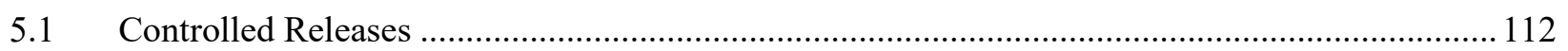

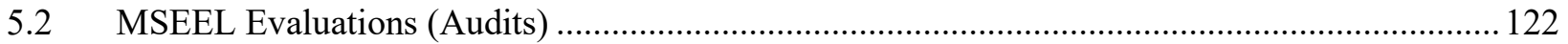

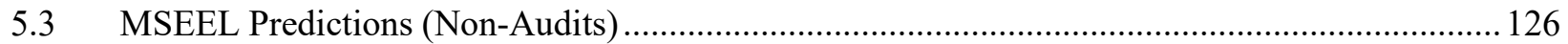

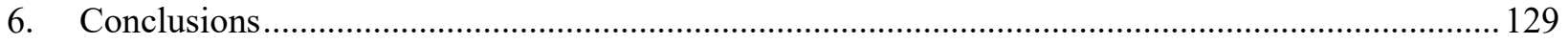

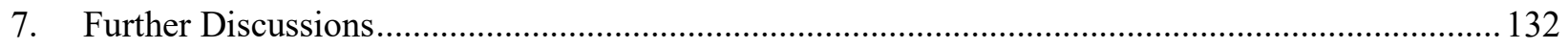

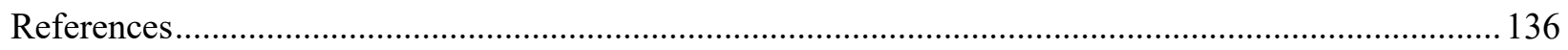

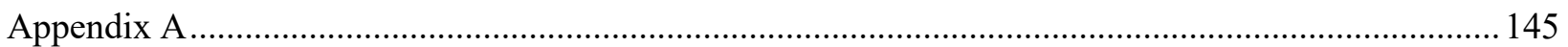




\section{Table of Tables}

Table 1. Sectors of natural gas supply chain and their respective components as defined by the EPA [8]..6 Table 2. US oil and gas production methane emissions by component source (2018) [20]..................... 7

Table 3. EPA GHGI data relevant to production sites and component emissions (2018) [24].................. 10

Table 4. Leak detection technologies and techniques as reviewed by Kimbrel et al. [38] ....................... 14

Table 5. Methane emissions from components at production sites measured by Pacsi et al [40] ............. 16

Table 6. Results and statistics of repeated methane measurements by Brantley et al. [50] ...................... 19

Table 7. OTM methane emission measurements and results by basin as reported by Brantley et al [50].. 19

Table 8. OTM methane emission measurements and results by basin reported by Robertson et al [51] ... 20

Table 9. OTM controlled release results as analyzed by Edie et al [53] ...............................................22

Table 10. OTM methane emission measurements, as reported by Robertson et al. 2020 [52]................ 23

Table 11. Controlled methane release results using mobile passes, as reported by Albertson et al. [56]... 24

Table 12. Field measurement results using mobile passes, as reported by Albertson et al [56]............... 24

Table 13. Indirect methane measurement campaigns and results for production sites $(\mathrm{kg} / \mathrm{hr})$...............22

Table 14. Summary of methane fluxes measured by EC from previous studies. .................................... 32

Table 15. Summary of footprint models and their performance as reviewed by Heidbach et al [80]........35

Table 16. Summary of aerial regional methane flux estimates from various studies. ............................. 38

Table 17. Methane measurement technologies from Stanford/EDF Mobile Monitoring Challenge [100].39

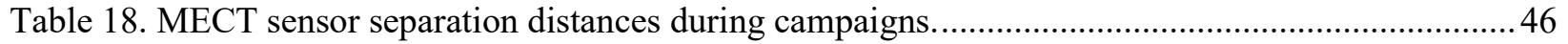

Table 19. Summary of major MECT equipment and their specifications [108] [112] ..........................4 47

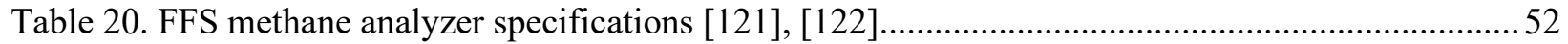

Table 21. FFS system results from various mass emission recovery tests............................................53

Table 22. MSEEL dimensions measured with Google Earth ${ }^{\circledR}$.........................................................5

Table 23. Component group bearings and distances relative to MECT location at MSEEL......................62

Table 24. Methane emissions from MSEEL direct quantification audits. .............................................63

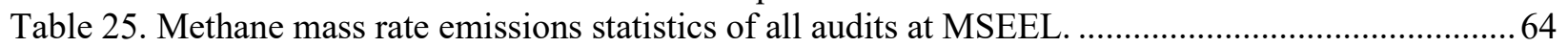

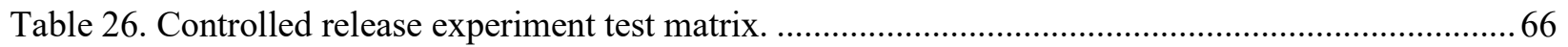

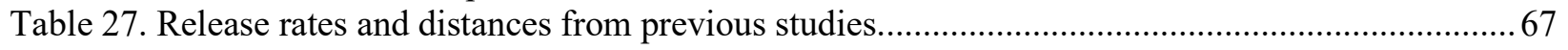

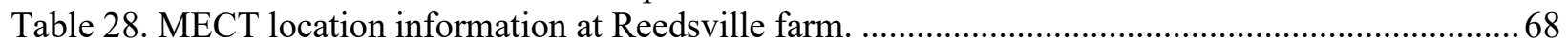

Table 29. Controlled release location information at Reedsville farm. ................................................... 68

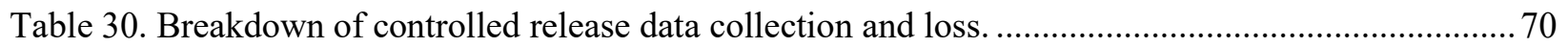

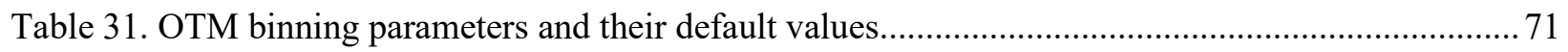

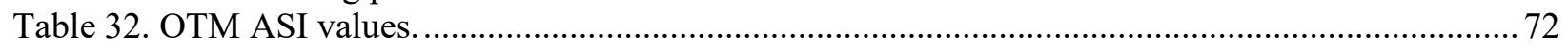

Table 33. Results from comparison between EPA MATLAB $®$ and Python analyses.............................. 73

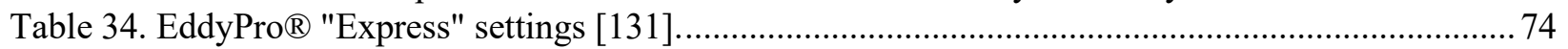

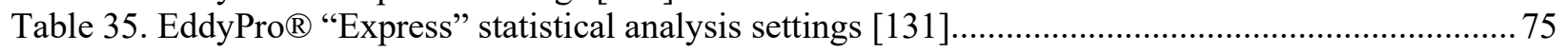

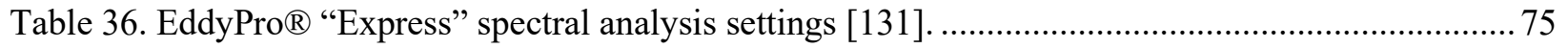

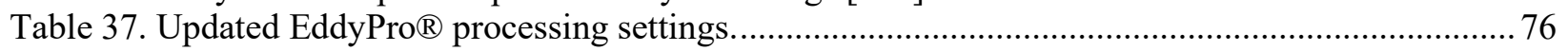

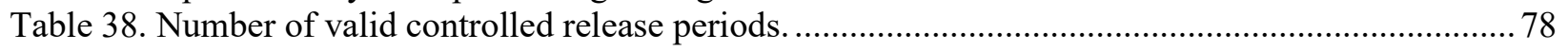

Table 39. Available periods from controlled releases for various filters. ............................................... 78

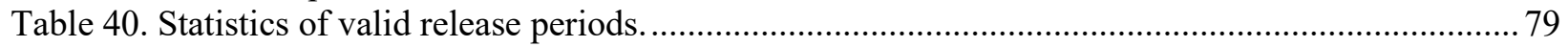

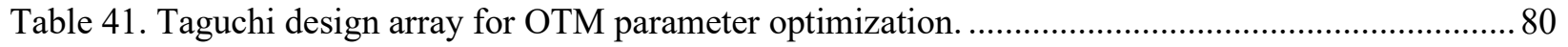

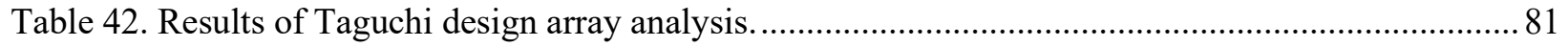

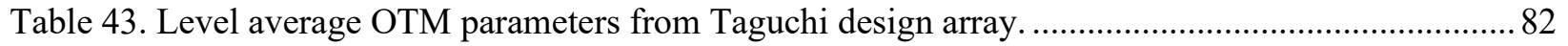

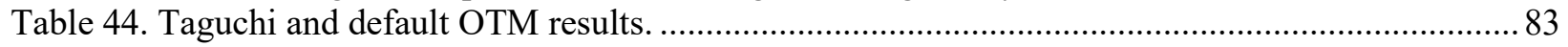

Table 45. Taguchi versus default OTM RMSE, across all controlled release scenarios. ......................... 83

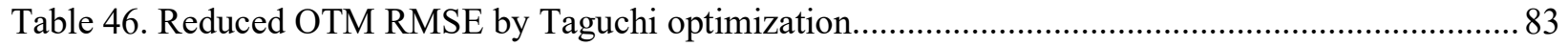

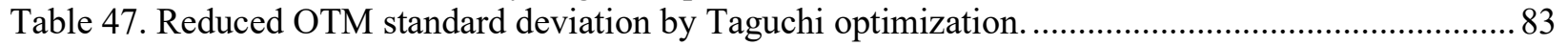

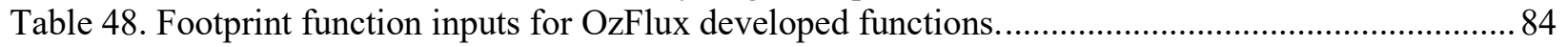

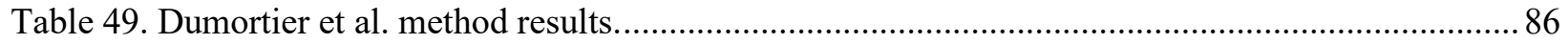


Table 50. Relevant OTM instrumentation information.

Table 51. Results of H\&R method applied to OTM measurements. ........................................................ 94

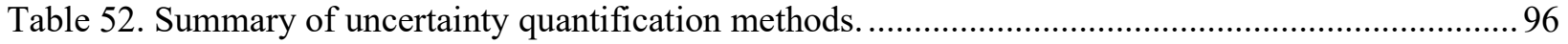

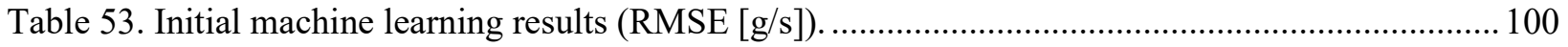

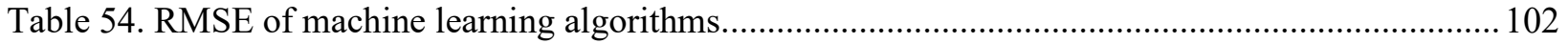

Table 55. Impact on model RMSE of adding individual features successively..................................... 106

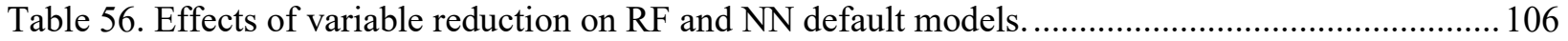

Table 57. Descriptions and scaling statistics of final model input features........................................... 108

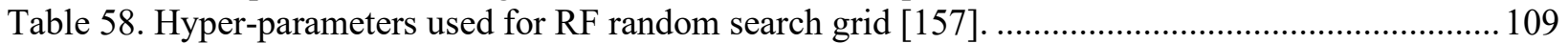

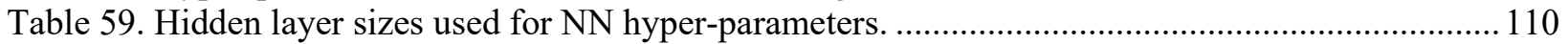

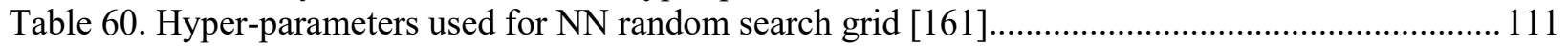

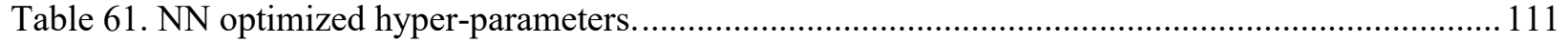

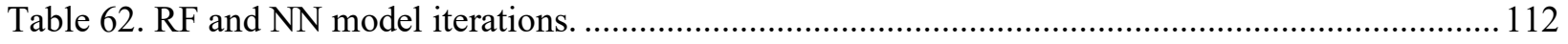

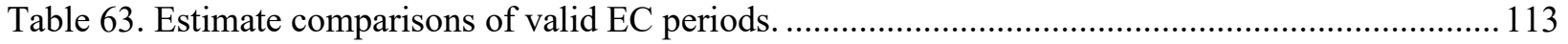

Table 64. Summary statistics of valid EC periods from all estimation methods.................................. 115

Table 65. RMSE results of full control release dataset for default and Taguchi OTM, RF, and NN...... 116

Table 66. Statistics of predicted rates (g/s) for default and Taguchi OTM, RF, and NN..................... 118

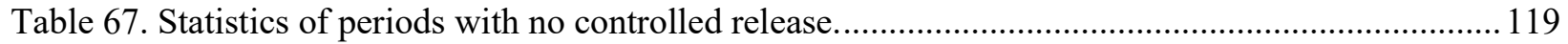

Table 68. Comparison of results to previous OTM studies (DQI < 10) . .......................................... 122

Table 69. Breakdown of available 15-minute periods from MSEEL. ............................................... 123

Table 70. RMSEs of MSEEL audits for default and Taguchi OTM, RF, and NN. ............................. 124

Table 71. Statistics of MSEEL predictions and audits....................................................................... 127 


\section{Table of Figures}

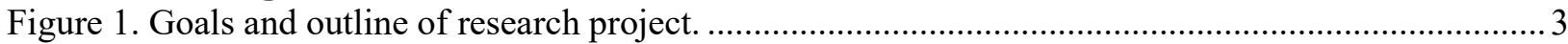

Figure 2. Historical increases in atmospheric methane concentration [10] .........................................5

Figure 3. Map of US natural gas producing wells clustered in shale plays (2020) [22] ......................... 7

Figure 4. Increasing trends in natural gas production, wells, and atmospheric methane.......................... 8

Figure 5. Projected natural gas withdrawals through 2050 for the US [23] ....................................... 8

Figure 6. US map of estimated methane emissions from production sites [26]..................................... 12

Figure 7. Comparison by Bell et al. of OTM and direct site measurement results [55]......................... 21

Figure 8. General representation of an EC application [65] ........................................................... 28

Figure 9. Temporal and spatial scales of methane detection and quantification technologies [104].......... 41

Figure 10. Summary of methane detection and quantification technologies reviewed by Fox et al [104]. 42

Figure 11. MECT deployed during controlled release experiments. .................................................... 44

Figure 12. MECT sensor separation schematic during data collection campaigns................................... 48

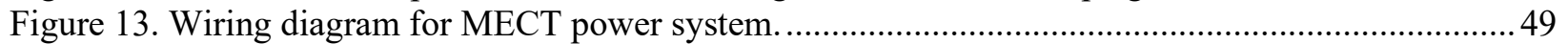

Figure 14. Wiring diagram of the MECT data acquisition system. ..................................................5

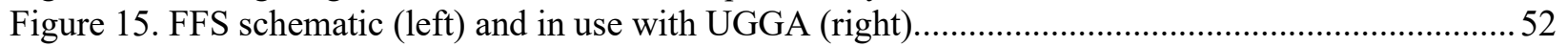

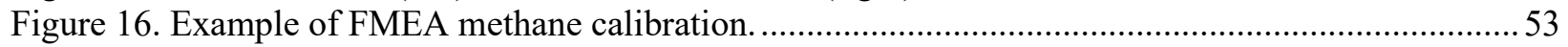

Figure 17. Example of MAF calibration against NIST traceable LFE. ..................................................5

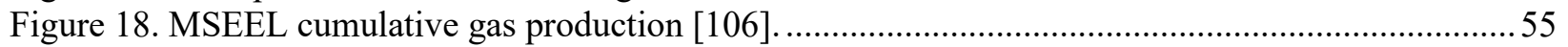

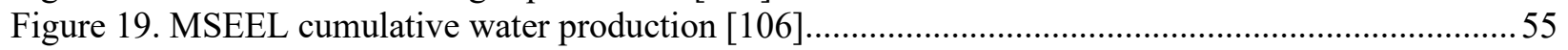

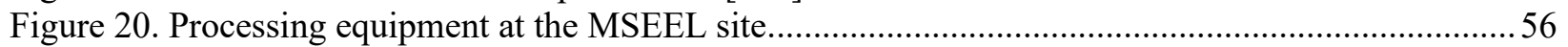

Figure 21. Layout of the MSEEL site (view from Google Earth $\left.{ }^{\circledR}\right)$ ) ....................................................56

Figure 22. Detection and sampling methods used during MSEEL audits. ...........................................59

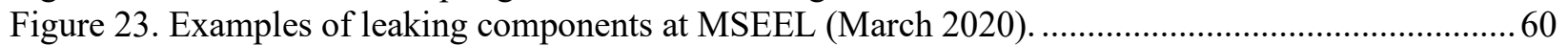

Figure 24. Distributions of methane mass emissions from the first 11 audits. ......................................61

Figure 25. MECT deployment relative to component groups at MSEEL .............................................61

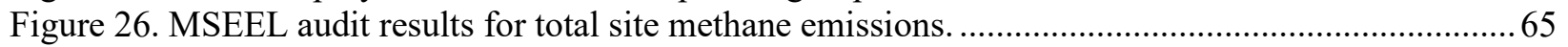

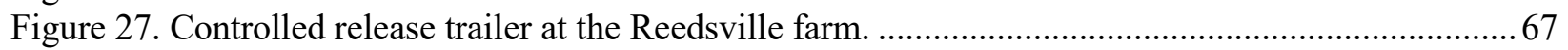

Figure 28. Map overview for controlled releases and MECT locations at Reedsville farm.....................69

Figure 29. Example EC footprints of FFP and KM, during controlled releases....................................... 85

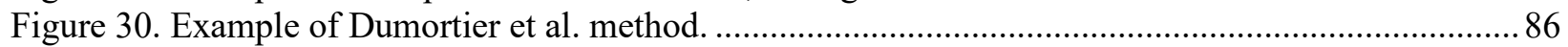

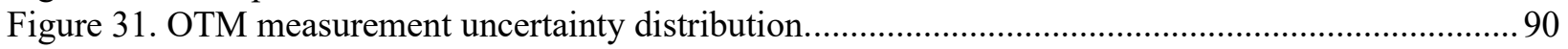

Figure 32. Bootstrapped OTM measurement uncertainty as a percentage of the estimate......................90

Figure 33. Bootstrapped mean FFSE of 776 periods. .......................................................................... 91

Figure 34. Distribution of flux pair differences used for EC H\&R uncertainty...................................... 93

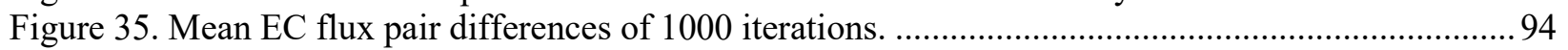

Figure 36. Comparison of traditional method results.................................................................. 95

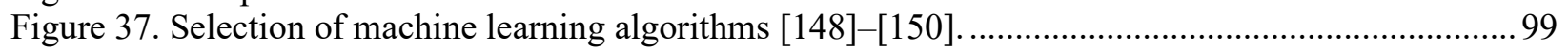

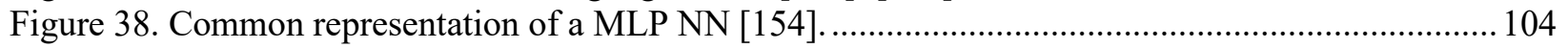

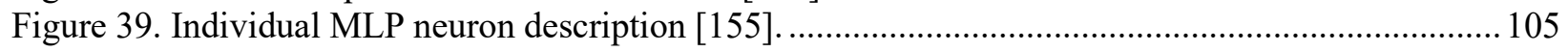

Figure 40. Comparison of method results for valid EC periods with a release rate of $0.04 \mathrm{~g} / \mathrm{s}$.............. 113

Figure 41. Comparison of method results for valid EC periods with a release rate of $0.12 \mathrm{~g} / \mathrm{s}$............... 114

Figure 42. Comparison of method results for valid EC periods with a release rate of $0.24 \mathrm{~g} / \mathrm{s} \ldots \ldots \ldots \ldots \ldots . .114$

Figure 43. RF test dataset mean IJ uncertainty. ............................................................................ 116

Figure 44. Comparison of default and Taguchi OTM, RF, and NN on all periods $(0.04 \mathrm{~g} / \mathrm{s}) \ldots \ldots \ldots \ldots \ldots . .117$

Figure 45. Comparison of default and Taguchi OTM, RF, and NN on all periods $(0.12 \mathrm{~g} / \mathrm{s}) \ldots \ldots \ldots \ldots \ldots . . .117$

Figure 46. Comparison of default and Taguchi OTM, RF, and NN on all periods $(0.24 \mathrm{~g} / \mathrm{s}) \ldots \ldots \ldots \ldots \ldots \ldots . . . .118$

Figure 47. Box and whisker of default and Taguchi OTM, RF, and NN background estimates............. 119

Figure 48. Low DQI percent error comparison for a controlled release rate of $0.04 \mathrm{~g} / \mathrm{s} \ldots \ldots \ldots \ldots \ldots \ldots \ldots \ldots . . . \ldots \ldots$

Figure 49. Low DQI percent error comparison for a controlled release rate of $0.12 \mathrm{~g} / \mathrm{s} \ldots \ldots \ldots \ldots \ldots \ldots \ldots . . . . . . . . .121$ 
Figure 50. Low DQI percent error comparison for a controlled release rate of $0.24 \mathrm{~g} / \mathrm{s}$. .

Figure 51. Comparison of default and Taguchi OTM, RF, and NN with MSEEL audits.....

Figure 52. MSEEL footprint heatmap of an example 15-minute period.

Figure 53. Box and whisker of OTM, RF, and NN predictions compared to MSEEL audit statistics. .... 127

Figure 54. Distributions of methods overlayed with audit measurements.

Figure 55. Predictions of MSEEL emission from various methods. 


\section{List of Equations}

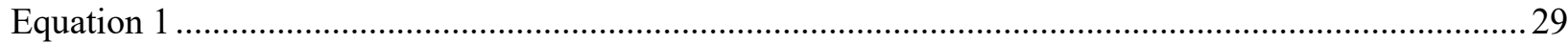

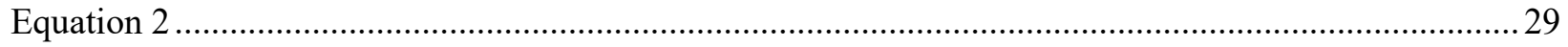

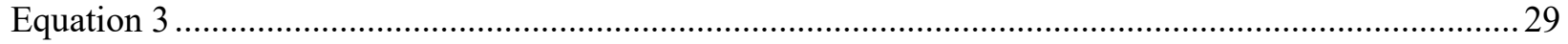

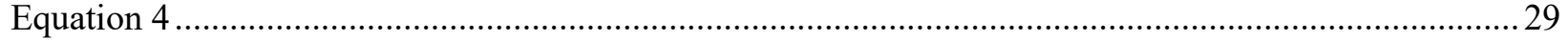

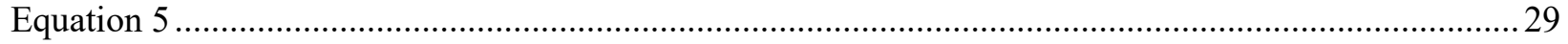

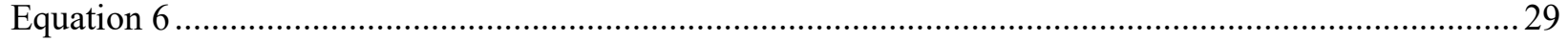

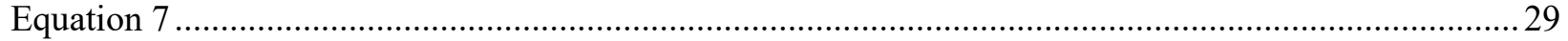

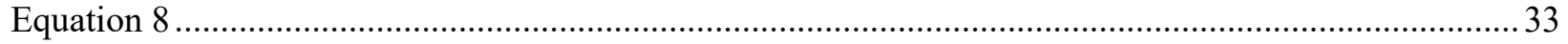

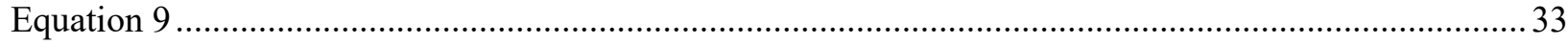

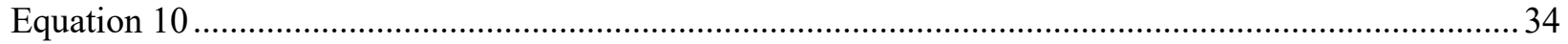

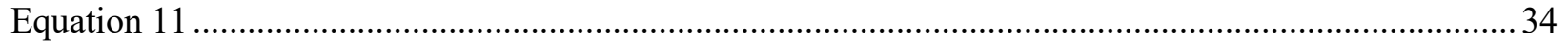

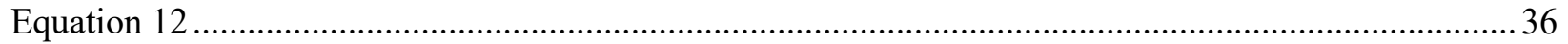

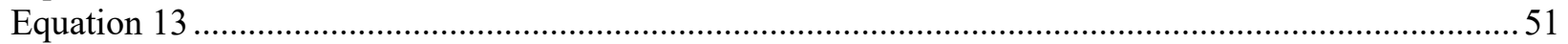

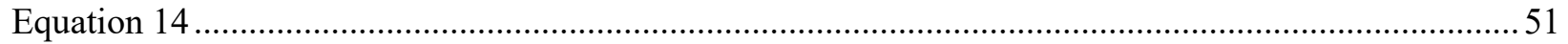

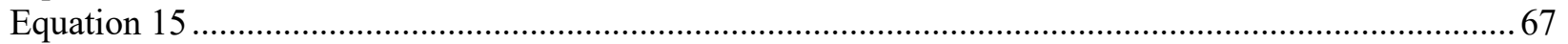

Equation 16

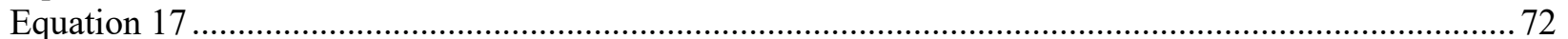

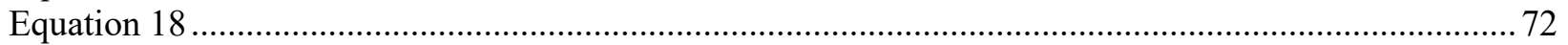

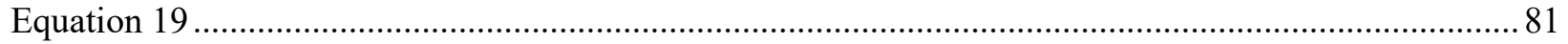

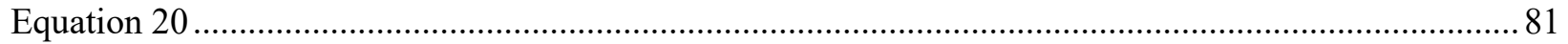

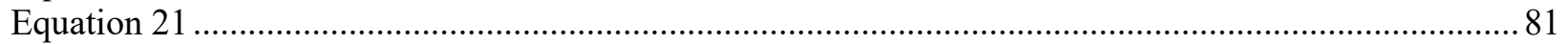

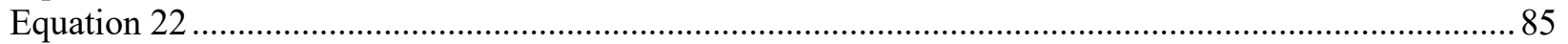

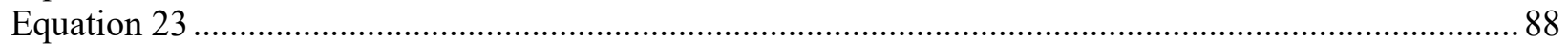

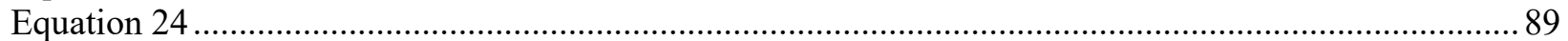

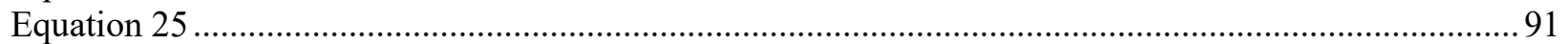

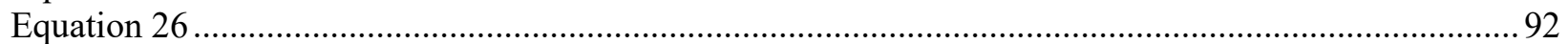

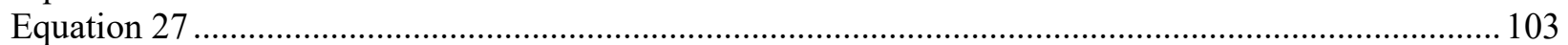

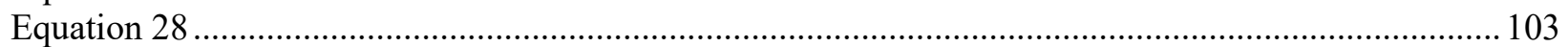

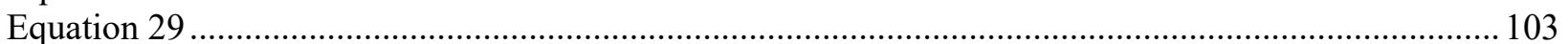

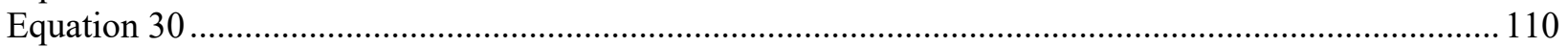

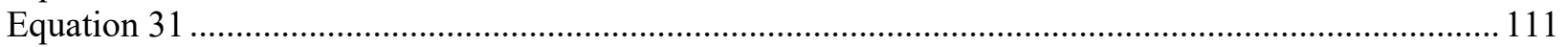




\section{List of Acronyms}

\begin{tabular}{|c|c|}
\hline${ }^{\circ} \mathrm{C}$ & degrees Celsius \\
\hline${ }^{\circ} \mathrm{EoN}$ & degrees East of North \\
\hline $\mathrm{AF}$ & activity factor \\
\hline ANN & Artificial Neural Network \\
\hline ARPA-E & Advanced Research Projects Agency - Energy \\
\hline ASI & atmospheric stability indicator \\
\hline bbl & barrels \\
\hline BDL & below detectable limit \\
\hline BU & bottom-up \\
\hline $\mathrm{CA}$ & California \\
\hline $\mathrm{CAF}$ & Christman Airfield \\
\hline CFR & Code of Federal Regulations \\
\hline $\mathrm{CH}_{4}$ & methane \\
\hline CI & confidence interval \\
\hline CL & confidence level \\
\hline CMI & concentration measurement instrument \\
\hline $\mathrm{CO}$ & Colorado \\
\hline $\mathrm{CO}_{2}$ & carbon dioxide \\
\hline $\mathrm{CO}_{2}-\mathrm{e}$ & carbon dioxide equivalent \\
\hline $\mathrm{CV}$ & Cross-fold Validation \\
\hline DAQ & data acquisition \\
\hline DJ & Denver-Julesburg \\
\hline DMS & Degrees-Minutes-Seconds \\
\hline DOE & Department of Energy \\
\hline DQI & data quality indicator \\
\hline E\&P & exploration and production \\
\hline EC & Eddy Covariance \\
\hline EDF & Environmental Defense Fund \\
\hline EDGAR & Emissions Database for Global Atmospheric Research \\
\hline $\mathrm{EF}$ & emissions factors \\
\hline EGPU & enclosed gas processing unit \\
\hline EIA & Energy Information Administration \\
\hline EPA & Environmental Protection Agency \\
\hline ERC & Engine Research Center \\
\hline $\mathrm{F} \& \mathrm{~S}$ & Finkelstein and Sims (2001) \\
\hline FFP & Kljun et al. (2015) Flux Footprint Prediction \\
\hline FFS & Full Flow Sampling \\
\hline FFSE & fractional flux sampling error \\
\hline FID & flame ionization detector \\
\hline FMEA & Fast Methane Ethane Analyzer \\
\hline
\end{tabular}




\begin{tabular}{|c|c|}
\hline $\mathrm{ft}$ & foot \\
\hline g & grams \\
\hline $\mathrm{g} / \mathrm{hr}$ & grams per hour \\
\hline$g / s$ & grams per second \\
\hline $\mathrm{g} / \mathrm{m}^{2}$-day & grams of carbon per square meter per day \\
\hline GDM & Gaussian dispersion model \\
\hline GHG & greenhouse gas \\
\hline GHGI & Greenhouse Gas Inventory \\
\hline GHGRP & Greenhouse Gas Reporting Program \\
\hline GMAP & Geospatial Measurement of Air Pollution \\
\hline GML & Global Monitoring Laboratory \\
\hline GPU & gas processing unit \\
\hline GPS & global positioning system \\
\hline GWP & global warming potential \\
\hline $\mathrm{H}_{2} \mathrm{O}$ & water \\
\hline$H \& R$ & Hollinger and Richardson (2005) \\
\hline $\mathrm{HCF}$ & high correlation filter \\
\hline $\mathrm{hr}$ & hour \\
\hline $\mathrm{Hz}$ & hertz \\
\hline IEA & International Energy Agency \\
\hline IJ & Infinitesimal Jackknife \\
\hline IMECC-EU & Infrastructure for Measurement of the European Carbon Cycle \\
\hline IPCC & Intergovernmental Panel on Climate Change \\
\hline IR & infrared \\
\hline K & Kelvin \\
\hline $\mathrm{kg}$ & kilograms \\
\hline $\mathrm{kg} / \mathrm{hr}$ & kilograms per hour \\
\hline KM & Kormann and Meixner (2001) footprint function \\
\hline $\mathrm{kPa}$ & kilopascals \\
\hline lbfgs & Limited-memory Broyden-Fletcher-Goldfarb-Shanno \\
\hline LDAR & Leak Detection and Repair \\
\hline LFE & laminar flow element \\
\hline LGR & Los Gatos Research \\
\hline LPDM-B & Lagrangian stochastic particle dispersion model \\
\hline LSM & Lagrangian stochastic model \\
\hline $\mathrm{m}$ & meters \\
\hline$m^{-2}$ & source area in square meters \\
\hline MAF & mass airflow sensor \\
\hline mbar & millibar \\
\hline MFC & mass flow controller \\
\hline MECT & Mobile Eddy Covariance Tower \\
\hline
\end{tabular}




\begin{tabular}{|c|c|}
\hline METEC & $\begin{array}{l}\text { Methane Emissions Technology Evaluation Center } \\
\text { megaorams }\end{array}$ \\
\hline $\mathrm{Mg}$ & megagrams \\
\hline MLP & Multi-Layer Perceptron \\
\hline $\mathrm{mm}$ & millimeters \\
\hline MMscf & million standard cubic feet \\
\hline MMT & million metric tons \\
\hline MQI & method quality indicators \\
\hline $\mathrm{m} / \mathrm{s}$ & meters per second \\
\hline MSEEL & Marcellus Shale Energy and Environment Laboratory \\
\hline Mt & metric tons \\
\hline $\mathrm{NaN}$ & Not a Number \\
\hline NETL & National Energy Technology Laboratory \\
\hline NG & natural gas \\
\hline NIST & National Institute of Standards and Technology \\
\hline NM & New Mexico \\
\hline $\mathrm{NN}$ & Neural Network \\
\hline NOAA & National Oceanic and Atmospheric Administration \\
\hline NSF & National Science Foundation \\
\hline OFE & OTM facility estimate \\
\hline OGI & optical gas imaging \\
\hline OLS & ordinary least squares \\
\hline OTM & Other Test Method \\
\hline PA & Pennsylvania \\
\hline $\mathrm{ppb}$ & parts per billion \\
\hline PPFD & Photosynthetic Photon Flux Density \\
\hline ppm & part per million \\
\hline PSG & point source Gaussian \\
\hline $\mathrm{R}^{2}$ & coefficient of determination \\
\hline relu & rectified linear unit \\
\hline $\mathrm{RF}$ & Random Forest \\
\hline RH & relative humidity \\
\hline RMLD & Remote Methane Leak Detector \\
\hline RMS & root mean square \\
\hline RMSE & root mean square error \\
\hline $\mathrm{RRC}$ & release rate correlated \\
\hline $\mathrm{Sc}$ & Schmidt number \\
\hline scfh & standard cubic feet per hour \\
\hline SE & standard error \\
\hline SI & Supporting Information \\
\hline sklearn & Sci-Kit Learn \\
\hline slpm & standard liters per minute \\
\hline
\end{tabular}




$\begin{array}{ll}\text { SOE } & \text { study onsite estimate } \\ \text { StS } & \text { Source to Sensor } \\ \text { Tcf } & \text { trillion cubic feet } \\ \text { TD } & \text { top-down } \\ \text { Tg } & \text { teragrams } \\ \text { TI } & \text { turbulence intensity } \\ \text { TX } & \text { Texas } \\ \mathrm{u}^{*} & \text { friction velocity } \\ \text { UAV } & \text { unmanned aerial vehicles } \\ \text { UGGA } & \text { Ultra-Portable Greenhouse Gas Analyzer } \\ \text { UNFCCC } & \text { United Nations Framework Convention on Climate Change } \\ \text { US } & \text { United States } \\ \text { VWLS } & \text { variance weighted least squares } \\ \text { WF } & \text { wind filtered } \\ \text { W/m2 } & \text { watts per square meter } \\ \text { WMS } & \text { Wavelength Modulation Spectroscopy } \\ \text { ws } & \text { wind speed } \\ \text { WV } & \text { West Virginia } \\ \text { WVU } & \text { West Virginia University } \\ \text { yr } & \text { year } \\ \mathrm{z}_{0} & \text { surface roughness } \\ \mathrm{z}_{\mathrm{m}} & \text { measurement height } \\ \mu \text { mol/mol } & \text { micromoles per mole (equivalent to ppm) } \\ \mu \mathrm{mol} / \mathrm{m}^{2} \mathrm{~s} & \text { microcmoles per square meter per second }\end{array}$




\section{Introduction}

The United States (US) is undergoing a fossil fuel transition. Over the past two decades the US has experienced a natural gas (NG) boom due to advances in exploration and production (E\&P) operations. The primary advancements have been horizontal drilling and hydraulic fracturing. The use of these methods has resulted in more efficient extraction of NG. These technologies have led to a rapid increase in the number of NG producing wells and production sites. The increase in NG production has resulted in a concern about methane emissions. Methane $\left(\mathrm{CH}_{4}\right)$ is the primary component of $\mathrm{NG}$ and a potent greenhouse gas (GHG). Some studies suggest that a significant amount of methane is escaping to the atmosphere due to the increased NG infrastructure. However, estimating the methane emissions from NG production has been difficult to achieve with a reliable level of accuracy. Many researchers and government agencies have produced estimates that differ by orders of magnitudes. To understand the environmental impact of the NG production industry, more reliable data need to be collected. Traditionally, methane emissions have been quantified through direct measurement techniques. However, as the number of production sites increases, this time-intensive method may no longer be economically viable for industry or researchers. In response, a few novel indirect quantification methods have emerged for use. These methods involve different scales (local and regional) and techniques (point measurement, mobile vehicle, aerial flux). The problem of uncertainty, however, is currently an issue for all indirect methods. More reliable methods need to be developed before indirect quantification can be relied upon by researchers, industries, and governmental policy makers and replace more accurate direct measurements. To increase the quality of indirect measurements several federally funded programs have been initiated in recent years. The National Science Foundation (NSF) recently awarded a grant to West Virginia University (WVU) that focused on the "intercomparison" of direct and indirect methane emissions measurements. The primary objectives were:

1. To directly quantify emissions temporally.

2. Develop and deploy a data acquisition (DAQ) system to record variables required for indirect measurements.

3. Employ machine learning to combine current methods with the goal of reducing indirect quantification uncertainty.

The work presented here is built around these primary objectives. The goal was to better understand the uncertainty of methane emissions measurements performed with two currently utilized stationary singlesensor techniques, Other Test Method (OTM) 33A and eddy covariance (EC). These methods both utilize a DAQ system with similar instrumentation and rely on atmospheric modeling principles to generate flux estimations. Multiple uncertainty analyses were performed on the datasets to gain an understanding of current limitations. In addition to these traditional techniques, more robust methods utilizing machine learning algorithms and output variables from traditional methods were explored. One of the objectives of the research was also to combine current methods with advanced algorithms. The hypothesis being that more consistent results could be achieved by combining similar but independent techniques. The hypothesis was tested through a series of controlled methane releases, allowing for comparison of traditional methods to the novel machine learning methods. The methodology was also applied to a real-world production site with uncontrolled emissions. Direct quantification audits known to be accurate and repeatable were utilized to periodically quantify methane emissions from the site. The machine learning methods were used to compare predicted emissions to those that were measured directly. Future measurements and research could continue to improve the methods applied here and will allow researchers to better understand the temporal uncertainty of sites without time and labor-intensive direct measurements.

To summarize:

- An up-tic in natural gas production and sites could be causing more methane emissions.

- Methane emissions are a potent GHG that need to be understood to be mitigated.

- Current techniques are often inaccurate and inconsistent, or cost intensive. 
- A DAQ system was developed to allow for simultaneous measurements of OTM and EC.

- A thorough uncertainty analysis was conducted to assess method limitations.

- Robust machine learning methods were developed which combined current techniques to improve emissions estimates.

- Tested against known releases and directly quantified emissions, machine learning methods provided more consistent and accurate methods of emissions estimation.

An overview of the research that was conducted is presented in Figure 1. 


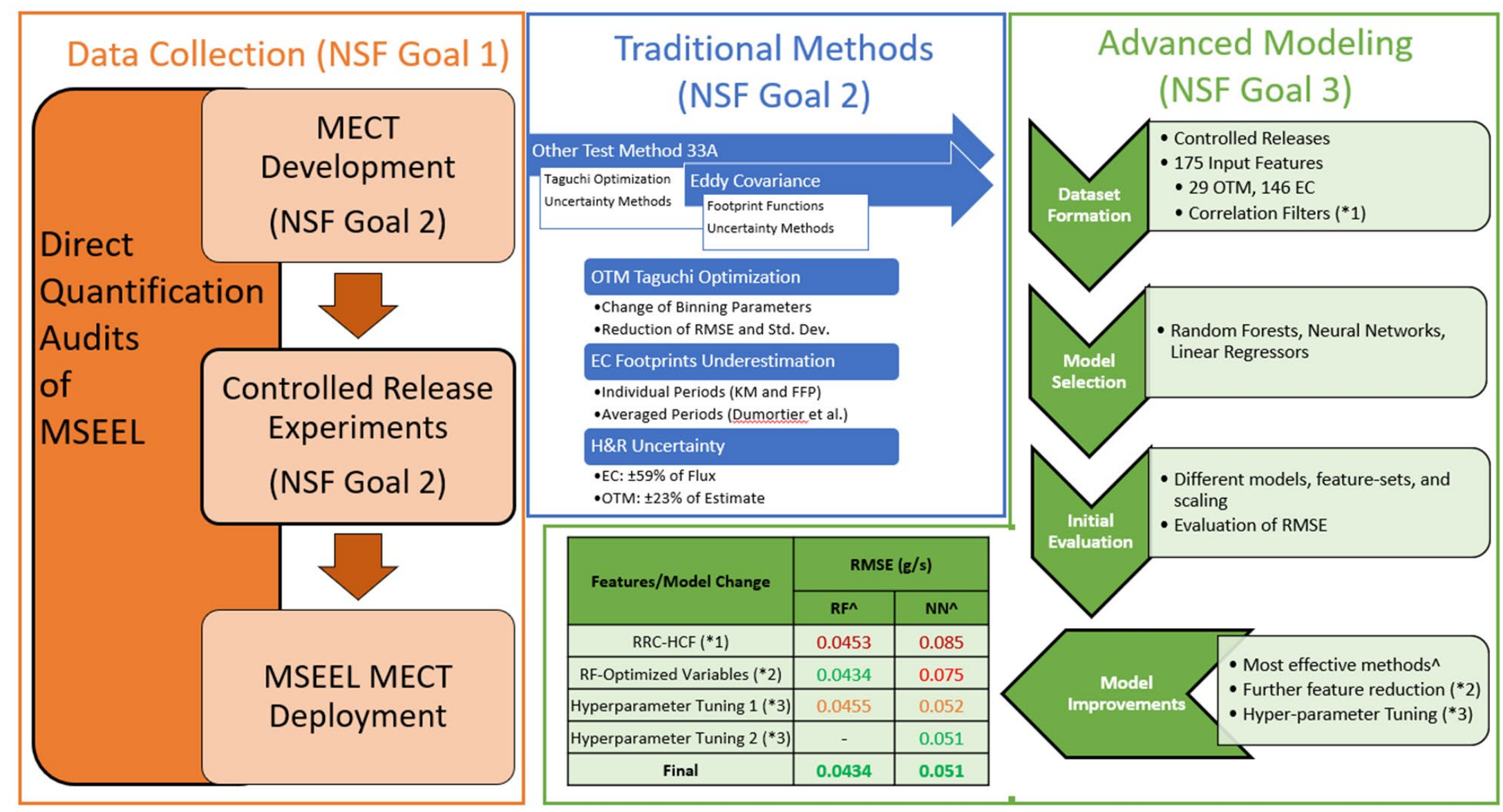

Figure 1. Goals and outline of research project. 


\section{Literature Review/Need for Research}

Atmospheric methane is considered a hazard to global warming. To better understand how to reduce atmospheric methane levels, knowledge of where methane emissions are emitted from is critical. As a result, there has been a recent push by regulators to reduce emissions, particularly those that are caused by humans - "anthropogenic" sources. One of the largest areas of methane emissions in the US is the NG infrastructure. Research conducted on NG infrastructure has resulted in a high level of uncertainty about the key sources and rates of emissions. As a result of this uncertainty and the need to better understand emissions profiles, several government agencies have recently funded programs focusing on various aspects of methane emissions quantification. The Environmental Defense Fund (EDF), Department of Energy (DOE) and their Advanced Research Projects Agency-Energy (ARPA-E), and the National Science Foundation (NSF) have all funded studies focused on the detection or quantification of methane emissions from the NG infrastructure in recent years [1]-[4]. The research conducted here was funded by the NSF under award number 1804024 "Inter-comparison of Direct Quantification and Areal Micrometeorological Methods to Investigate the Transport and Fate of Methane from Heterogenous Sources in Natural Gas Fields". The primary objective of this research was to "advance the science of direct and indirect measurement techniques" [5].

\subsection{Methane Emissions and the Natural Gas Industry}

Methane has recently gained attention from researchers, regulators, and industry due to its potency as a greenhouse gas (GHG). The scientific consensus is that GHGs are the primary cause of the Earth's greenhouse effect. The greenhouse effect is what allows the Earth to maintain a livable atmosphere, however, excessive quantities of GHGs in the atmosphere is believed to be responsible for increased global temperatures. The measure of a gas' ability to trap heat in the atmosphere is its global warming potential (GWP). The GWP is calculated by integrating the impulse response in radiative forcing caused by a given gas and dividing by the integrated value of carbon dioxide's $\left(\mathrm{CO}_{2}\right)$ impulse response. This gives $\mathrm{CO}_{2}$ a GWP of one and all other gases are compared to this standard value. The integration can be carried out over different time periods or "time horizons" often defined as 20 or 100 years. The time horizon used has a large impact on the value assigned to a given gas. The 100-year time horizon was adopted as the standard by the United Nations Framework Convention on Climate Change (UNFCCC). According to the Intergovernmental Panel on Climate Change (IPCC) the most up to date (2013) estimates on the GWP of methane are 86 and 34 over the 20- and 100-year periods, respectively, when including climate-carbon feedbacks. The GWPs reduce to only 84 and 28, respectively, when these feedbacks are excluded [6]. The IPCC previously reported a methane GWP of 25 over the 100 -year period and this number is still commonly used. The Environmental Protection Agency (EPA) currently uses this value in their GHG inventories (GHGI), and reports GHG emissions in terms of million metric tons of $\mathrm{CO}_{2}$ equivalent (MMT $\left.\mathrm{CO}_{2}-\mathrm{e}\right)$ [8]. Note that one MMT is equivalent to $10^{9}$ kilograms $(\mathrm{kg})$ or 1 teragram $(\mathrm{Tg})$.

The rise in temperature of the Earth may result in significant changes to the planets ecosystem. It is therefore important to minimize the level of GHGs in the atmosphere. Despite these presumptions, atmospheric methane levels have been increasing rapidly since continuous measurements began in 1984 by the National Oceanic and Atmospheric Administration's (NOAA) Global Monitoring Laboratory (GML) [9]. The atmospheric methane concentration in 2020 was $6 \%$ higher than levels just 20 years ago. These levels are believed to be the highest in Earth's recent history (approximately 800,000 years). It is believed that preindustrial atmospheric methane concentration never exceeded one part per million (ppm). However, current levels exceed $1.8 \mathrm{ppm}$, as presented in Figure 2. 


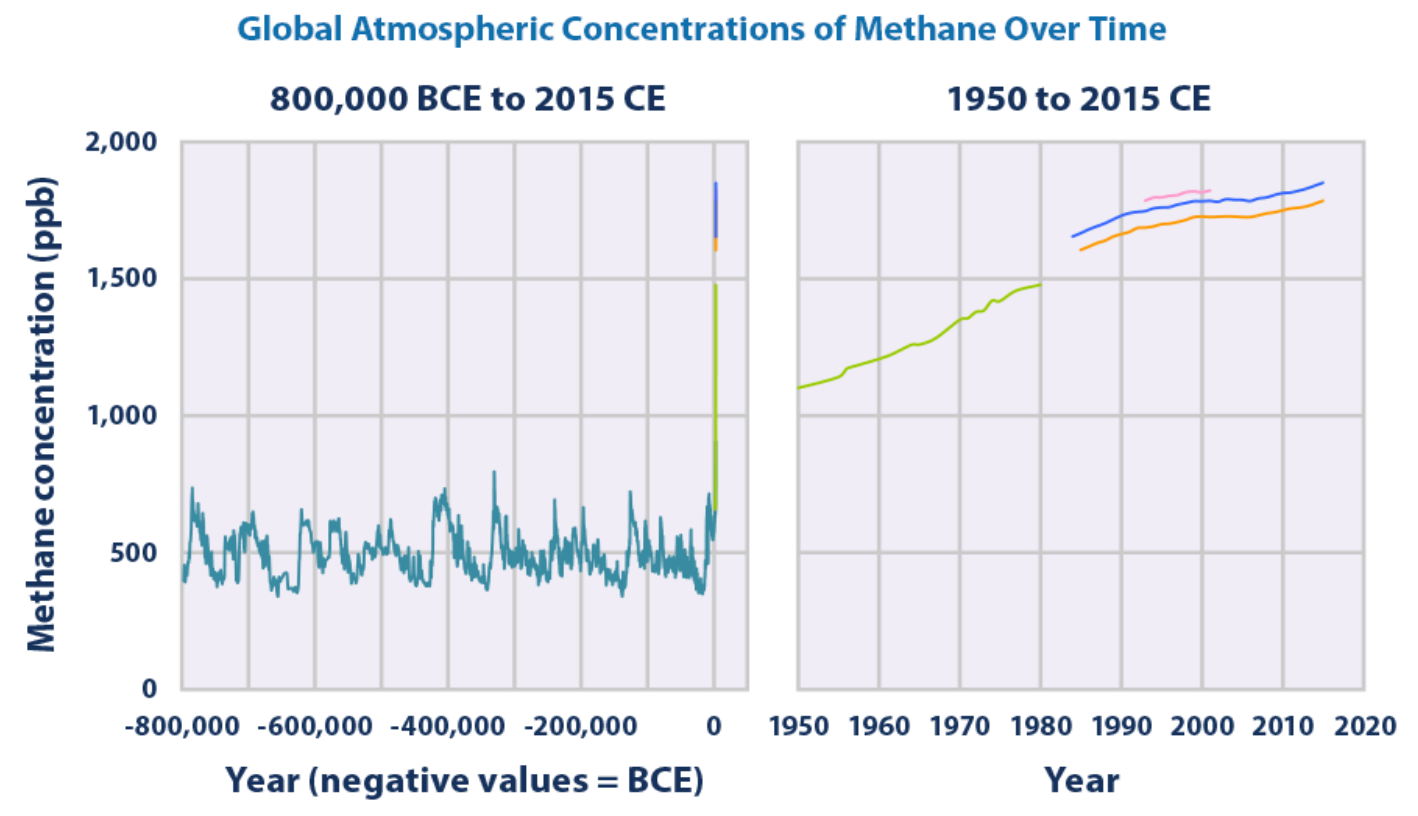

Data source: Compilation of five underlying datasets. See www.epa.gov/climate-indicators for specific information.

For more information, visit U.S. EPA's "Climate Change Indicators in the United States" at www.epa.gov/climate-indicators.

Figure 2. Historical increases in atmospheric methane concentration [10].

When GHG emissions are due to human related activity they are considered "anthropogenic". Examples of anthropogenic sources of methane include landfills, oil and NG production, agriculture, and fuel combustion [11]. Estimations for the total methane budget could be as high as $400 \mathrm{Tg}$ of carbon per year [13]. Best estimates suggest 50-65\% of these global methane emissions are anthropogenic [12]. Nonanthropogenic or "biogenic" emissions of methane include those produced in the stomachs of ruminants (enteric fermentation) and seepage from shallow coal and oil deposits, wetlands, and other ecosystem components and processes. The US EPA estimates that methane makes up $10 \%$ of all GHG emissions in the US and $16 \%$ of all GHG emissions globally. The largest anthropogenic sources of methane include NG and petroleum systems (28\%), enteric fermentation (28\%) and landfills (17\%) [11]. The International Energy Agency (IEA) estimated that there were 82 million tonnes of methane emissions from the oil and gas industry in 2019. About half of these emissions are estimated to be from NG [14]. The EPA estimated that in 2018 total NG system methane emissions were about $140 \mathrm{MMT} \mathrm{CO}_{2}$-e, the second highest source behind enteric fermentation [15].

According to the EPA's latest estimates about $47 \%$ of the total oil and gas methane emissions ( 175 MMT $\mathrm{CO}_{2}$-e) are from gas production alone. Emissions from the production segment are estimated to be about $117 \mathrm{MMT} \mathrm{CO}_{2}$-e. The EPA defines the production sector as the activities associated with NG extraction, gathering, and boosting. Therefore, this section does not include only "production" or "well" sites but also gathering and boosting stations and other intermediary sites.

The natural gas supply chain is often broken down when defining the problem of methane emission in one of two ways. The entire supply chain may be divided by defining the "upstream" and "downstream" portions. When using this definition, the primary "upstream" sources of emissions include E\&P activities, active and abandoned wellheads, and other leaks and losses associated with the equipment found on production sites and other facilities managed by the E\&P companies. Activities in the "downstream" portion include compression, gathering, boosting, processing, and distribution. Sometimes a third portion known as "midstream" will be included in this type of breakdown, when this definition is included, it typically 
encapsulates most "downstream" activities other than some processing and distribution. Another way of breaking down the supply chain is by sector. The EPA defines the sectors of the NG supply chain as Production, Processing, Transmission and Storage, and Distribution. The EPA estimates nationwide emissions from these sectors in its annual greenhouse gas inventory (GHGI). This report is updated annually with data form the greenhouse gas reporting program (GHGRP) and other data from industry and researchers. A breakdown of what is included in each sector according to the EPA's GHGI is presented in Table 1 [8].

Table 1. Sectors of natural gas supply chain and their respective components as defined by the EPA [8].

\begin{tabular}{|c|c|c|c|}
\hline Production & Processing & Transmission \& Storage & Distribution \\
\hline $\begin{array}{ll}\text { - } & \text { Gas Wells } \\
\text { - } & \text { Well Pad Equipment } \\
\text { - } & \text { Produced Water from Coal } \\
& \text { Bed Methane } \\
\text { - } & \text { Normal Operations } \\
& \quad \text { Pneumatics) } \\
\text { - } & \text { Condensate Tank Vents } \\
\text { - } & \text { Compressor Exhaust Vented } \\
\text { - } & \text { Well Clean Ups } \\
\text { - } & \text { Blowdowns } \\
\text { - } & \text { Upsets } \\
\text { - } & \text { Gathere } \\
\end{array}$ & 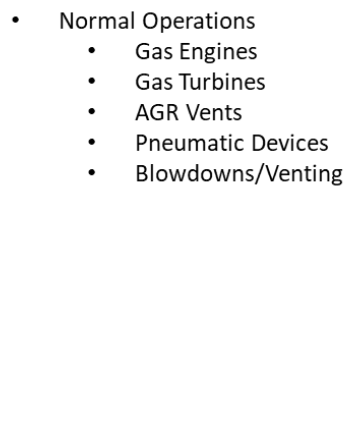 & 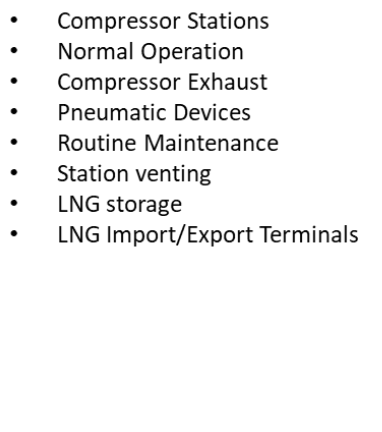 & $\begin{array}{ll}\text { - } & \text { Pipeline leaks } \\
\text { - } & \text { Meter/Regulator (City Gates) } \\
\text { - } & \text { Customer Meters } \\
\text { - } & \text { Routine Maintenance } \\
\text { - } & \text { Upsets }\end{array}$ \\
\hline
\end{tabular}

The primary focus of this research was on NG production sites. Production sites are also commonly referred to as "well sites", "well pads", or "production pads". Production sites contain at least one gas producing wellhead. The wellhead is the surface component of a well which contains the equipment necessary to control the pressure of the drilled well. In terms of emissions, it is often synonymous with well, as each wellhead corresponds to an individually drilled well. With the recent improvements in drilling and completion technology many production sites now contain several wellheads. At the beginning of the shale gas boom a half a decade ago 3-4 wellheads to a site was considered large, however, nowadays it is common to see more than 10 wellheads per site [16]. Some production sites may contain as many as 35 wellheads [17].

In addition to wellheads, production sites also contain several other components that support the initial extraction and processing of NG. NG is generally separated from sand and water at the production site so many sites contain a variety of sand and liquid separators. Typical NG production sites also contain gas processing units (GPUs), gas and water scrubbers, dehydrators, treaters, and heaters. These components require piping, pneumatic valves, pressure and temperature monitors, flanges for connections and other additional infrastructure and components. In addition, production sites typically house a variety of tanks for produced liquids. These tanks may contain crude oil, water, or condensates depending on the quality of the NG produced by the well. Produced water and condensate tanks contain the water and NG liquids that are separated from the NG at the production site. Such tanks can be major sources of emissions from flashing, breathing or general operation [18]. Produced water tanks are typically left open to the atmosphere, while NG liquid tanks are typically sealed. NG liquid tank emissions are regulated and controlled through various methods such as flaring; however, water tanks are not. In 2016 researchers surveyed 17 unconventional production sites and found 1-9 wells per pad with an average of 5 wells per pad. Each site averaged 5 GPUs, 6 liquid storage tanks, buffer tanks, recovery units, and enclosed burners [19]. All the components on a production site through which methane is transferred are potential emissions sources, either by the nature of their operation or through faulty equipment. The distribution of emissions from these production sources as estimated by the EPA is presented in Table 2 [20]. 
Table 2. US oil and gas production methane emissions by component source (2018) [20].

\begin{tabular}{|l|c|}
\hline Emission Source & $\mathbf{\%}$ \\
\hline Pneumatic Controllers & 37 \\
\hline Gathering and Boosting Stations & 27 \\
\hline Offshore Platforms & 5 \\
\hline Chemical Injection Pumps & 4 \\
\hline Liquids Unloading & 4 \\
\hline Completions and Workovers & 2 \\
\hline Tanks & 2 \\
\hline Other & 20 \\
\hline
\end{tabular}

The number of production sites in the US has increased dramatically over the course of the NG boom of the early $21^{\text {st }}$ century, particularly in the second decade. US NG production hit an all-time high in 2019 at just under 40.9 trillion cubic feet (Tcf). Production was $71 \%$ greater in 2019 than it was 20 years prior and $57 \%$ greater than it was 10 years prior. The number of gas producing wells peaked in 2014 at just over 580,000 wells. From 2014 to 2019 the number of producing wells decreased to just over 490,000 wells which is equivalent to the number in 2009 . However, this number still represented a $62 \%$ increase from 1999 levels [21]. Currently active US production sites are presented in Figure 3. Figure 4 presents the growth of NG production in terms of wells and gross withdrawals over the past two decades, along with the rise in atmospheric methane levels.

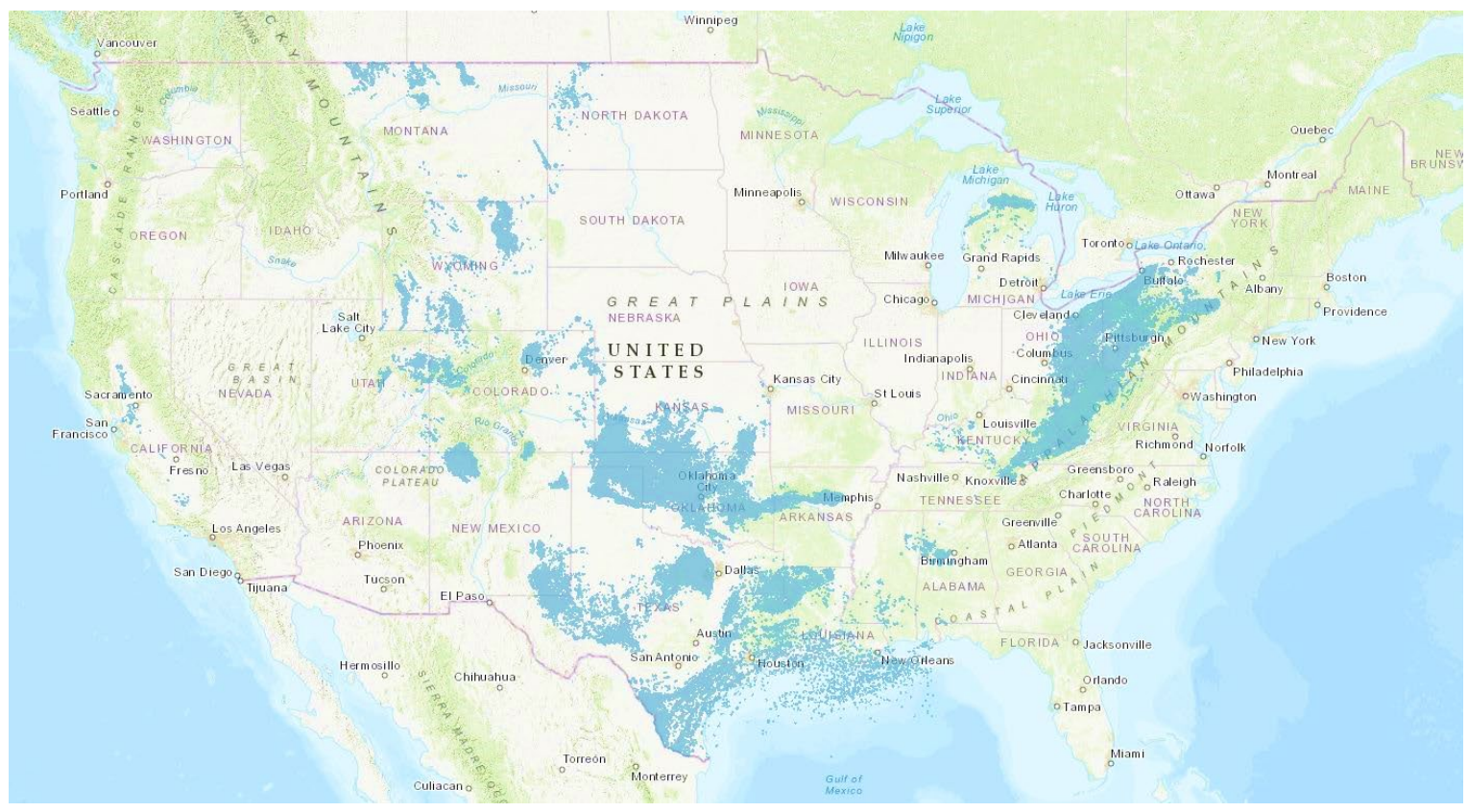

Figure 3. Map of US natural gas producing wells clustered in shale plays (2020) [22]. 


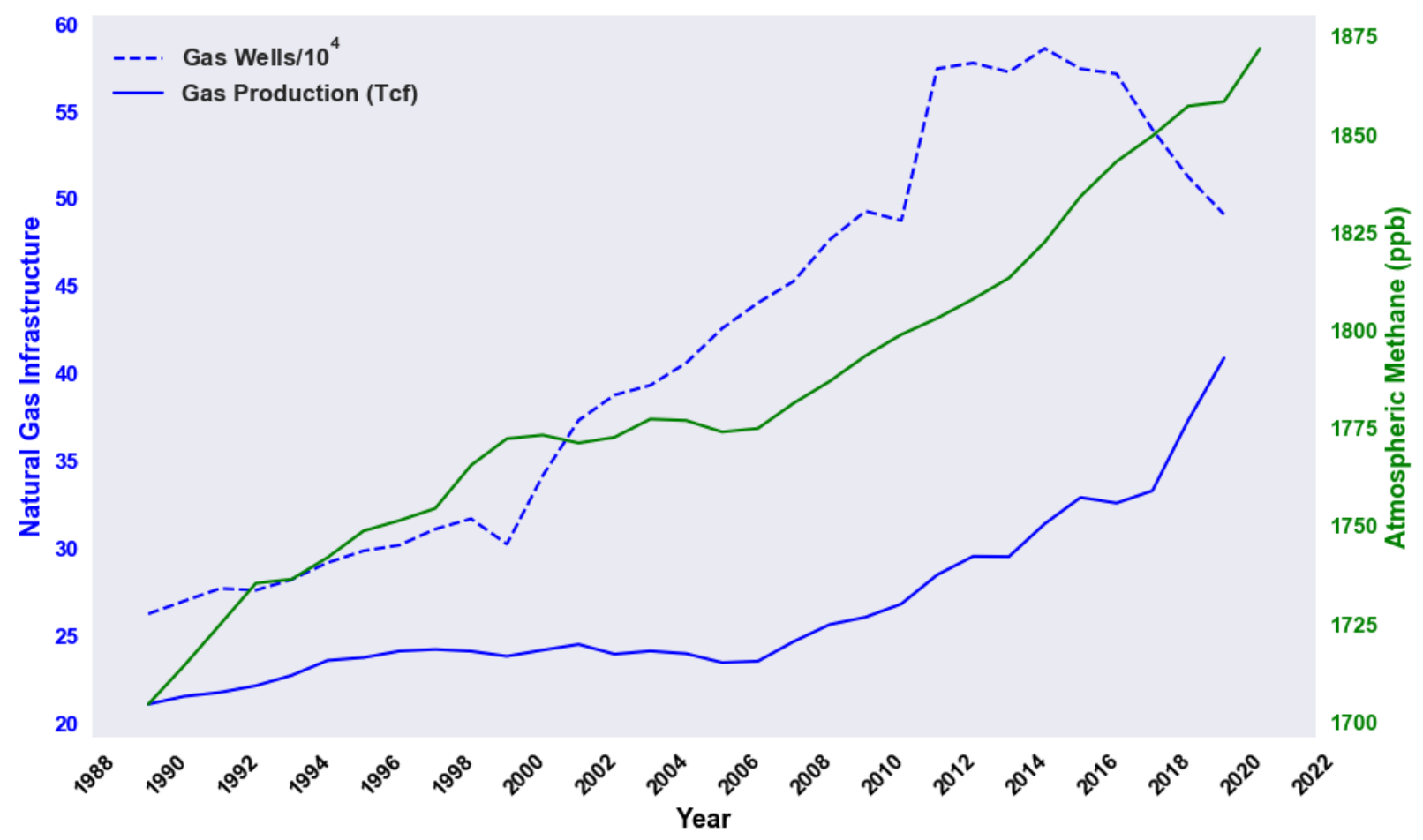

Figure 4. Increasing trends in natural gas production, wells, and atmospheric methane.

Figure 4 displays a clear correlation between NG production and atmospheric methane levels. While the number of production sites has declined in recent years, NG production continues to climb and is projected to do so until 2050 according to the EIA, as presented in Figure 5.

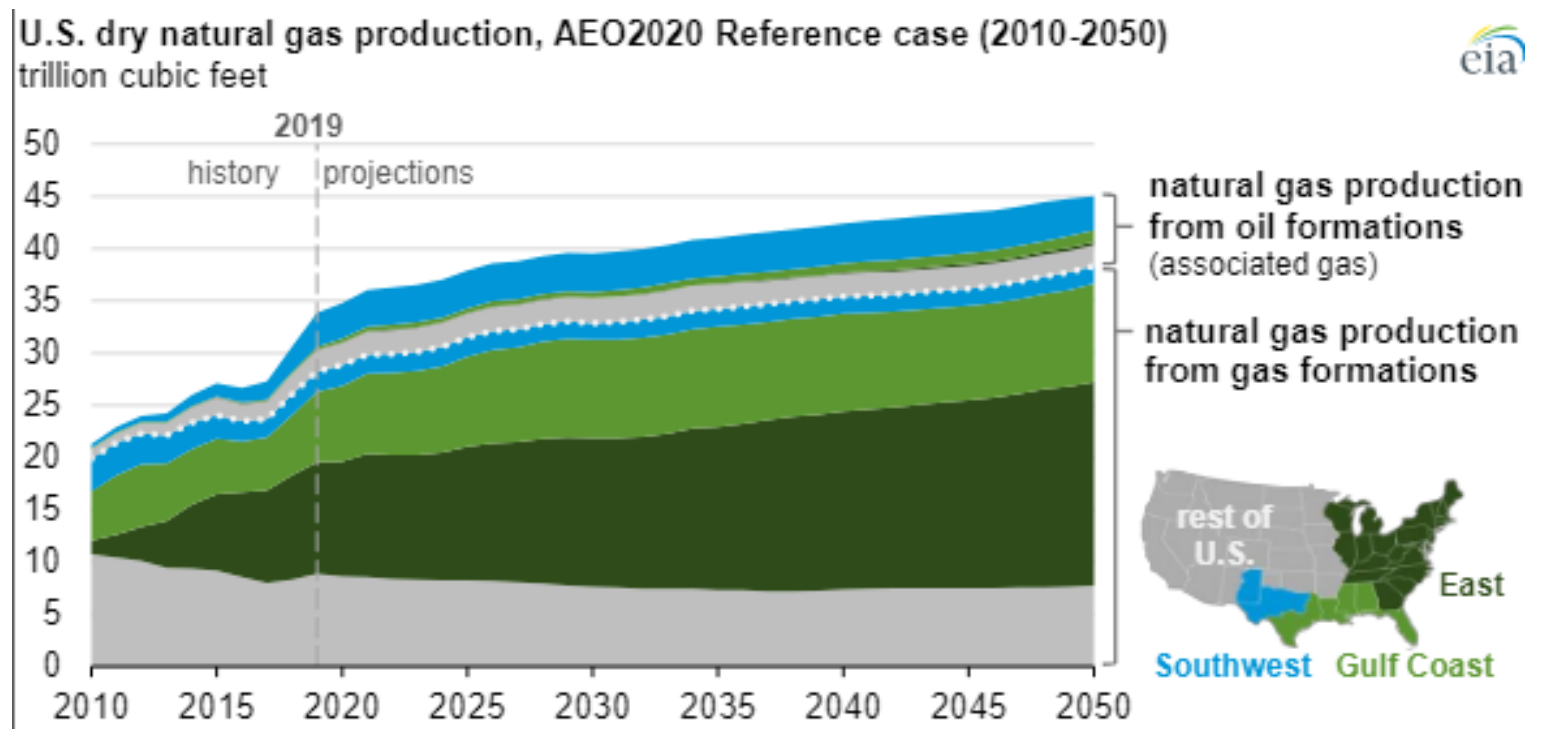

Figure 5. Projected natural gas withdrawals through 2050 for the US [23]. 


\subsubsection{Disparities in Emissions Estimates}

Several studies have attempted to quantify the total methane emissions lost across the NG supply chain. There are two primary methods for developing such an estimation. These two methods of measurement are generally referred to in literature as bottom-up (BU) and top-down (TD). BU estimates rely on direct measurements from components. This data is then used to develop emissions factors (EF) - a rate of mass emissions due to that component's operation. These emissions factors are combined with best guesses as to the number of these components on a given site and the number of sites in the country, known as activity factors (AF). Regional and national estimates can then be made by multiplying the emissions factors (mass emissions / component) by the activity factors (\# of components) to get the total mass emissions from a given sector, component, or type of site. These methods rely heavily on numbers reported to regulators by researchers and industry. Historically there has been no clear standard of reporting. The most recent report (2020) contains estimates from the NG industry from 1990 through 2018 [24]. For NG systems the report is subdivided by the segments presented in Table 1. The production sector is broken down into many subcategories also presented in Table 1. Emissions from production sites (well pads) are a portion of the total emissions from the production sector and are generally dominated by the equipment on site. Activity factors, emissions factors, and total emissions relevant to production sites estimated from the GHGI 2020 report are presented in Table 3. Some emissions and activity factors still in use by the EPA in 2020 are based on data that was collected as far back as 1992 [8].

It is not possible for researchers, regulators, or industry to measure every site component. As such the direct measurements used in making BU estimates are a small subset of all sites and components. While these may be representative, it has been shown that methane emissions from the NG industry sites and components often have fat-tailed distributions [25]-[27]. If an inventory estimate excluded the high emitters or includes too many of them, then the compounded emissions estimates will contain large errors. Emissions have also been shown to be highly temporal [28], [29]. This fact alone makes it nearly impossible to develop accurate EFs for a given component or site with a small number of direct measurements.

TD estimates rely heavily on indirect quantification techniques, such as regional tower or aerial flux estimates, or more recently satellite concentration measurements. These estimates are generally produced from changes in atmospheric methane concentration. Large areas can be covered with aerial type measurements; however, it can be difficult to attribute emissions to a specific source. Some methods, such as ethane correlations, have been used to differentiate anthropogenic from biogenic methane when utilizing these techniques. This does not solve the issue of targeting emissions sources for reduction, particularly in the NG sector where infrastructure density is high in basins with high production. Differentiating between sources of emissions within the NG infrastructure is critical to reducing emissions. 
Table 3. EPA GHGI data relevant to production sites and component emissions (2018) [24].

\begin{tabular}{|c|c|c|c|c|c|}
\hline Segment/Source & EF & Units & $\mathbf{A F}(\#)$ & Units & Emissions (kt) \\
\hline \multicolumn{3}{|l|}{ Gas Wells } & 405,026 & wells & \\
\hline Non-associated Gas Wells (less fractured wells) & 89.4 & $\mathrm{~kg} / \mathrm{well}$ & 165,719 & wells & 14.8 \\
\hline Gas Wells with Hydraulic Fracturing & 137.7 & $\mathrm{~kg} / \mathrm{well}$ & 239,307 & wells & 32.9 \\
\hline \multicolumn{6}{|l|}{ Well Pad Equipment } \\
\hline Heaters & 244.2 & $\mathrm{~kg} / \mathrm{heater}$ & 53,055 & heaters & 13.0 \\
\hline Separators & 392.4 & $\mathrm{~kg} /$ separator & 287,691 & separators & 112.9 \\
\hline Dehydrators & 470.5 & $\mathrm{~kg} /$ dehydrator & 12,028 & dehydrators & 5.7 \\
\hline Meters/Piping & 206.8 & $\mathrm{~kg} / \mathrm{meter}$ & 339,571 & meters & 70.2 \\
\hline Compressors & $1,990.4$ & $\mathrm{~kg} /$ compressor & 32,619 & compressors & 64.9 \\
\hline \multicolumn{6}{|l|}{ Normal Operations } \\
\hline Pneumatic Device Vents & $1,248.5$ & $\mathrm{~kg} /$ controller & 814,075 & controllers & $1,016.4$ \\
\hline (Low Bleed) & 160.6 & $\mathrm{~kg} /$ controller & 210,845 & controllers & 33.9 \\
\hline (High Bleed) & $4,370.7$ & $\mathrm{~kg} /$ controller & 19,991 & controllers & 87.4 \\
\hline (Intermittent Bleed) & $1,534.7$ & $\mathrm{~kg} /$ controller & 583,239 & controllers & 895.1 \\
\hline Chemical Injection Pumps & $1,521.2$ & $\mathrm{~kg} / \mathrm{pump}$ & 71,903 & active pumps & 109.4 \\
\hline Kimray Pumps & 20.2 & $\mathrm{~kg} / \mathrm{MMscf}$ & $3,520,422$ & MMscf & 71.1 \\
\hline Dehydrator Vents & 5.6 & $\mathrm{~kg} / \mathrm{MMscf}$ & $3,951,091$ & MMscf & 22.2 \\
\hline \multicolumn{6}{|l|}{ Condensate Tank Vents } \\
\hline Large Tanks w/Flares & 0.005 & $\mathrm{~kg} / \mathrm{bbl}$ & $128,694,626$ & bbl & 0.6 \\
\hline Large Tanks w/VRU & 0.005 & $\mathrm{~kg} / \mathrm{bbl}$ & $15,801,742$ & bbl & 0.1 \\
\hline Large Tanks w/o Control & 0.2 & $\mathrm{~kg} / \mathrm{bbl}$ & $37,370,309$ & bbl & 6.1 \\
\hline Small Tanks w/Flares & 0.01 & $\mathrm{~kg} / \mathrm{bbl}$ & $8,495,905$ & bbl & 0.1 \\
\hline Small Tanks w/o Flares & 0.6 & $\mathrm{~kg} / \mathrm{bbl}$ & $25,668,215$ & bbl & 16.5 \\
\hline Malfunctioning Separator Dump Valves & 0.0001 & $\mathrm{~kg} / \mathrm{bbl}$ & $181,866,677$ & bbl & 0.02 \\
\hline \multicolumn{6}{|l|}{ Compressor Exhaust Vented } \\
\hline Gas Engines & $4,813.4$ & $\mathrm{~kg} / \mathrm{MMHPhr}$ & 51,855 & MMHPhr & 249.6 \\
\hline
\end{tabular}


In addition to the EPA GHGI, the European Commission maintains the Emissions Database for Global Atmospheric Research (EDGAR), which tracks the atmospheric levels of several gas pollutants, including methane. Several studies have raised issues with these intergovernmental estimates, stating that they generally underestimate emissions.

Howarth et al. estimated gross loss of NG from shale plays in 2011 to be 3.6-7.9\% over its lifecycle (from extraction to end use). This estimate was 1.5-3.0 times higher than the EPA's estimate of 2.4\% [30].

Alvarez et al. estimated that national methane emissions from the oil and gas supply industry could be as high as $13 \mathrm{Tg} / \mathrm{yr}$. This number was $60 \%$ higher than the corresponding GHGI estimate of $8.1 \mathrm{Tg} / \mathrm{yr}$. The error bounds of these estimates did not overlap, suggesting that uncertainty in estimates was high. The differences in emissions estimates could be even more drastic in specific segments. Emissions estimates from the production sector data have historically been drastically different. The EPA's most recent estimate of $3.5 \mathrm{Tg} / \mathrm{yr}$ was less than half of the 2012 study's estimate of $7.6 \mathrm{Tg} / \mathrm{yr}$. This study also estimated a total emission rate from production sites in the US to be approximately $830 \mathrm{Mg} / \mathrm{hr}$ [31].

In 2013 Miller et al. estimated that US methane emissions reported by the EPA and EDGAR could be 1.5 and 1.7 times lower than actual values. Miller et al. also emphasized that regional estimates could be even more erroneous. For example, their research suggested that emissions from fossil fuel sources in the southcentral US could be $4.9 \pm 2.6$ times higher than EDGAR estimates [13].

Brandt et al. conducted an extensive review of published estimates in 2014 and concluded that studies show emissions from the NG infrastructure were generally 1.5 times higher than EPA estimates [32]. The researchers suggested that this underestimation could be as high as $14 \mathrm{Tg} / \mathrm{yr}$. The researchers emphasized that many emissions estimates are prone to errors and acknowledged that BU studies are often skewed by under-representing "super-emitters" due to small sample sizes or the use of out-of-date measurement technologies. TD studies based on atmospheric measurements suffer from the inability to attribute observations to sources, especially without isotopic measurements. A 2014 review by Allen concurred that BU emissions inventories underestimated emissions compared to TD studies [33].

In 2015, Zavala-Araiza et al. utilized a combination of BU and TD estimates from the 2013 EDF coordinated Barnett shale campaign and found that the mean difference in these estimates was $0.1 \% \pm 21 \%$. However, their estimated emissions from oil and gas methane were $90 \%$ higher than the GHGI. The researchers estimated total BU methane to be $59 \mathrm{Mg} / \mathrm{hr}(95 \% \mathrm{CI}: 48-73 \mathrm{Mg} / \mathrm{hr})$ with the primary source being production sites at $53 \%$ of total emissions. This estimate was 1.9 times greater than the GHGI, 3.5 times greater than the GHGRP, and 5.5 times greater than EDGAR [34].

A more recent 2018 study by Vaughn et al. included concurrent TD and BU measurements in the Fayetteville region. Their results showed overlapping 95\% CIs but underestimation from BU techniques. The measurements were taken on two different days and divided the region into halves but consistently found that BU approaches underestimated by $5-20 \%$. The total estimate for the region was $0.25 \mathrm{Tg} / \mathrm{yr}(28.7$ $\mathrm{Mg} / \mathrm{hr})[28]$.

Omara et al. have conducted multiple studies on NG production site emissions [19], [26]. In 2018 researchers performed national and regional emissions estimates of NG production sites in the US based on data from several studies. The researchers analyzed data of more than 1000 NG production sites. They estimated that the total emissions rate from approximately 498,000 US production sites was $830 \mathrm{Mg} / \mathrm{hr}$ (95\% CI: 530-1200) based on a non-parametric model. This total was 2.3 times higher than the EPA's GHGI. The researchers also estimated West Virginia (WV) and Pennsylvania (PA) region emissions as 144 $\mathrm{Mg} / \mathrm{hr}$ and $115 \mathrm{Mg} / \mathrm{hr}$ in 2016 and 2018, respectively [26]. It is important to note that the study focused only on sites with "normal" operation. They did not include emissions from events such as flowbacks or liquids unloadings which are known to contribute large quantities of emissions. The range of estimates spanned from 0 to $300 \mathrm{~kg} / \mathrm{hr}$ with $74 \%$ of sites emitting between 0.1 and $10 \mathrm{~kg}$ of methane per hour. The 
highest $5 \%$ of emitters contributed $57 \%$ of the emissions. Researchers have since referred to this as the 5 50 rule. The average emissions rate of the study was more than $13 \mathrm{~kg} / \mathrm{hr} / \mathrm{site}$. The research divided sites by production volume. They estimate that none of the 220,000 production sites that produced less than 10 Mcfd were in the top $5 \%$, with an average emission rate of $0.46 \mathrm{~kg} / \mathrm{hr} / \mathrm{site}$. Figure 6 presents a map of emissions estimates from the study.

Both TD and BU studies that use indirect quantification have unfavorable qualities. TD models must make assumptions about the sources of emissions, use highly stochastic atmospheric transport models with high uncertainty, and often lack validation data from direct measurements. BU studies often have high variability in reported emissions and small-scale flux calculations often do not translate to regional scales.

One of the reasons cited by Zavala-Ariza et al. for the erroneous estimates of regulators was the extreme "spatially-temporal" nature of "super-emitters". The study showed that at any one time $2 \%$ of facilities are responsible for $50 \%$ of emissions and $10 \%$ are responsible for $90 \%$. The research also suggested that production sites were more likely to be high emitters than other NG sites and that $30 \%$ of production sites emit more than $1 \%$ of the NG they produce. This rate of methane loss accounted for more than $70 \%$ of all production site emissions [34]. The fact that emissions are not normally distributed even when a significant sample is obtained also makes it difficult to estimate the emissions of an entire region or nation. ZavalaAraiza et al. noted in a separate study the fat-tailed distribution caused by "super-emitters" and determined that $15 \%$ of sites are likely to be "super-emitters" at any given time. The defined "super-emitting" sites as the highest $1 \%$ of sites measured, which had an average mass rate of $26 \mathrm{~kg} / \mathrm{hr}$ [25]. Four individual studies have all estimated that the highest $5 \%$ of emitting sites contribute more than $50 \%$ of the total methane emissions from production [25], [26], [32], [35].

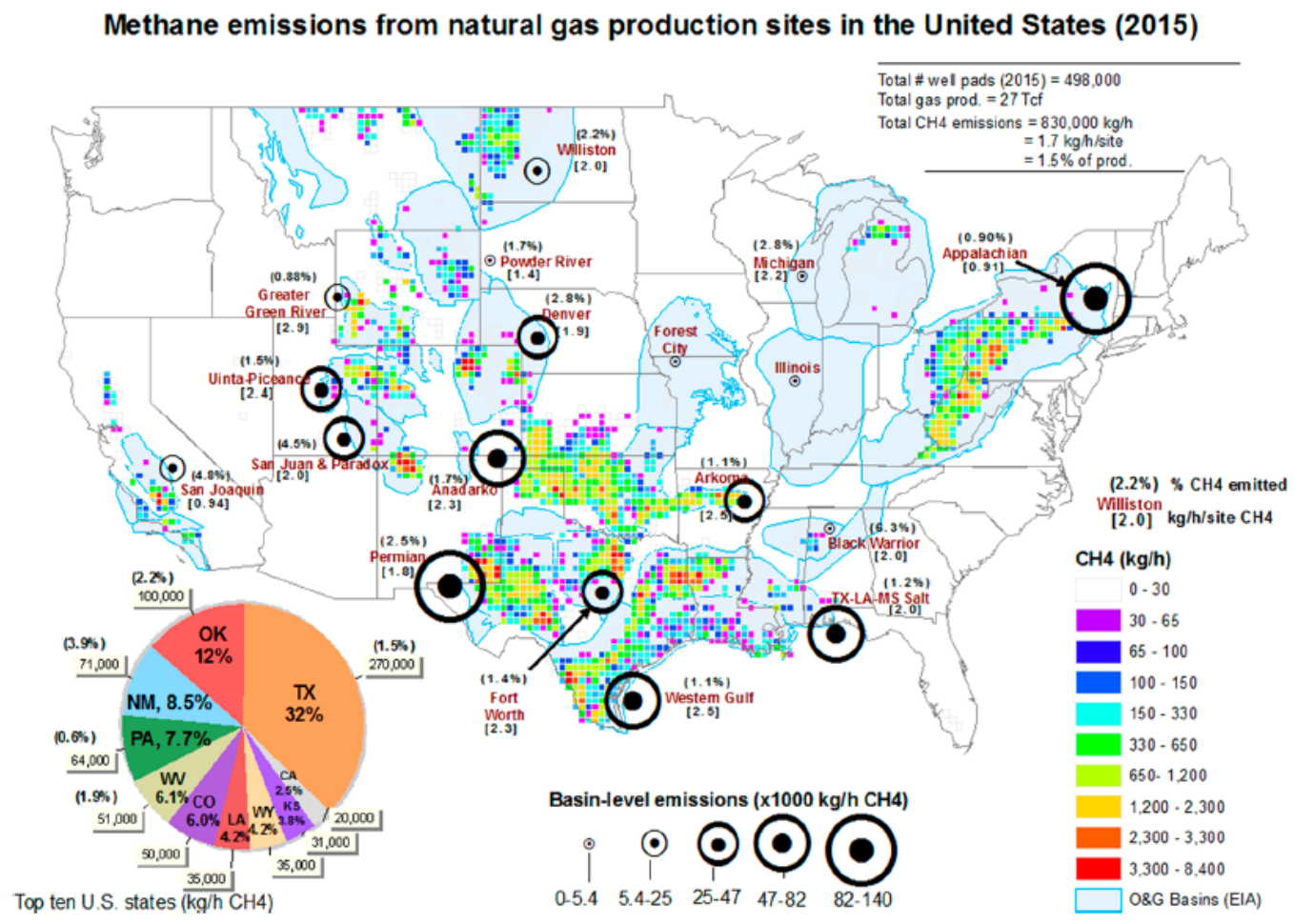

Figure 6. US map of estimated methane emissions from production sites [26]. 


\subsection{Methane Measurement Methods}

Clearly there are disparities between emissions measurements on a large scale such as regional or national. This is due to two primary contributions, uncertainty in local emissions quantification measurements and uncertainty in activity factors. The second issue is resolvable and will continue to improve as companies begin to better maintain databases on the number wells drilled and overall infrastructure. The number of abandoned and unaccounted for wells will surely decrease in the coming years as companies become more conscious of the environmental and monetary impacts. The first contributor, the uncertainty of local emissions measurements, continues to be a problem for researchers and industry alike due to the difficulty inherent in performing accurate emissions measurements effectively and economically. Several of the methods of emissions quantification are outlined here.

\subsubsection{Detection}

Unfortunately for those attempting to determine total emissions from the NG industry, the most common type of emissions survey currently implemented does not involve quantification. Leak Detection and Repair (LDAR) programs are generally maintained by the NG industry either proactively or reactively. There are several methods used by industry and researchers to detect NG leaks and the area of NG leak detection has grown rapidly over the past few years and continues to be an area of active research. Recent reviews of methane leak detection technologies have identified several existing and emerging methods and technologies. Most LDAR programs are governed by EPA Method 21, defined by the Code of Federal Regulations (CFR) [36], [37]. This method requires the use of a portable device which can measure volatile organic compounds (VOCs) to detect leaks from on-site equipment. The limit of detection for a methane leak is defined by the EPA as 500 parts per million (ppm).

Measuring every device on-site using Method 21 with a handheld device is a time intensive procedure, so the EPA now allows the use of Optical Gas Imaging (OGI) cameras as an alternative in many instances. Using OGI cameras is quicker but does not allow for any estimation of the leak rate. Method 21 does suggest a quantification calculation, though it does not measure the emissions rate directly, but instead infers it from a curve fit of concentration and leak rate defined in the CFR. To infer a mass rate from concentration requires that the concentration measurements be made in the same way as those used to develop the correlation. The uncertainty of the method is therefore a product of user error.

The requirements for most NG sites are defined in the CFR Section OOOO [36]. These requirements vary by the type of NG operation performed at the site in question. Most well pads require semi-annual evaluations with either Method 21 or OGI. If Method 21 is used, any leak detected above the 500-ppm threshold must be reported. If OGI is used any detectable emissions must be reported. Some states have more stringent regulations. California (CA), Colorado (CO), and PA require quarterly audits for production sites with either Method 21 or OGI.

Kimbrel et al. performed a recent (2019) review of newer and emerging NG leak detection technologies. The report categorized technologies as either "existing" or "emerging" [38]. The leak detection technologies reviewed by the authors are presented in Table 4. While this list presents some of the more reviewed methods, it is not an exhaustive list of currently available technologies. 
Table 4. Leak detection technologies and techniques as reviewed by Kimbrel et al. [38]

\begin{tabular}{|c|c|c|c|}
\hline Technology & Status & $\begin{array}{c}\text { Primary Manufacturer / } \\
\text { Developer }\end{array}$ & Technique \\
\hline Flame Ionization Detector (FID) & Existing & Many & Direct \\
\hline Infrared (IR) Cameras & Existing & FLIR (and others) & Imaging \\
\hline High-Volume Dilution Sampling & Existing & Previously Bacharach ${ }^{\mathrm{TM}}$ & Direct \\
\hline $\begin{array}{l}\text { Remote Methane Leak Detector } \\
\text { (RMLD-IS }\end{array}$ & Existing & Heath Consultants Inc. & Remote \\
\hline Mobile Leak Detection & Existing & $\mathrm{ABB}$ & Remote \\
\hline Calibrated Bag & Existing & Dupont with Tedlar® & Direct \\
\hline $\begin{array}{l}\text { Satellite Imaging and Remote } \\
\text { Sensing }\end{array}$ & Existing & Many & Imaging/Remote \\
\hline $\begin{array}{l}\text { Aerial Light Detection and } \\
\text { Ranging System }\end{array}$ & Emerging & Bridger Photonics & Remote \\
\hline Advance Leak Detection Lidar & Emerging & Ball Aerospace & Remote \\
\hline Gas Cloud Imaging Camera & Emerging & Rebellion Photonics & Imaging \\
\hline Gas Tracer & Emerging & RKI Instruments & Direct \\
\hline GAZOSCARNTM $^{T}$ & Emerging & GazoMat & Remote \\
\hline $\begin{array}{l}\text { IRwin }{ }^{\circledR} \text { Portable pump-based } \\
\text { infrared sensor }\end{array}$ & Emerging & Inficon & Direct \\
\hline Laser Methane Mini $^{\mathrm{TM}}$ & Emerging & Tokyo Gas & Remote \\
\hline MIRA PICO Mobile LDS & Emerging & Aeris Technologies & Remote \\
\hline MobileGuard $^{\mathrm{TM}}$ & Emerging & $\begin{array}{l}\text { ABB/Heath Consultants } \\
\text { Inc. }\end{array}$ & Remote \\
\hline Picarro Surveyor ${ }^{\mathrm{TM}}$ & Emerging & Picarro Incorporated & Remote \\
\hline $\begin{array}{l}\text { QM3000 Continuous Methane } \\
\text { Detection System }\end{array}$ & Emerging & Quanta3 & Remote \\
\hline RMLD-CSTM & Emerging & Heath Consultants Inc. & Remote \\
\hline SeekIR TM $^{\text {TM }}$ & Emerging & SeekOps & Remote \\
\hline
\end{tabular}

There are three broad methods of leak detection technologies. These methods are imaging, remote concentration measurement, and direct concentration measurement. Imaging techniques involve the use of infrared cameras. These methods can be rendered ineffective by weather, background infrastructure, and low temperature gradients. Indirect detection techniques often utilize a point sensor or infrared laser method. Paired with other micrometeorological measurements or mobile platforms these measurement methods can be effective but are not well established. The most reliable detection methods are those that use direct measurement; however, these methods are time and resource intensive, and the effectiveness often relies on the operator. These methods utilize a device that can measure the gas concentration by sampling a mixture of gas and air, often with a pump. Devices are generally operated manually, and individual components must be scanned for long enough to obtain a steady value. Leaks are identified by a concentration reading on the device above a specified level. This is the method most widely accepted by regulators and used for Method 21. These techniques sometimes have the advantage of not only identifying leaks but quantifying them. With the proper equipment and operation these methods are highly accurate $(<5 \%$ error). However, there are far fewer methods of direct quantification than there are leak detection. Well established methods include high flow sampling and vacuum bagging. Even with these techniques it can be difficult to ensure that the entire leak is captured and quantified, which can lead to underestimation. 


\subsubsection{Direct Quantification}

Historically, the most accurate method of quantification has been through direct measurement. The two primary methods of direct measurements are high volume sampling and vacuum bag sampling. Several studies have utilized direct measurements for the purpose of quantifying emissions from NG production sites. These campaigns have focused on individual components (Allen et al. - pneumatic controllers), regions (Kuo et al. - California), the entire supply chain (Pacsi et al.) or specific components in a region (Kang et al. - abandoned wells in the Marcellus region) [39]-[42]. Most direct quantification campaigns then rely on emissions and activity factors to perform nationwide or regional estimates because the sample size is generally small compared to indirect methods.

Pneumatic controllers are of interest due to their operational nature. A pneumatic controller is designed to regulate flow, level, or pressure and will use gas either intermittently or continuously to regulate these processes. This means that during normal operation they will vent NG emissions to the atmosphere. Typically, production sites contain several pneumatic controllers per well head. Allen et al. has performed several direct quantification campaigns that have emphasized pneumatics at production sites. During one campaign presented data from 33 individual production sites that had an average of $3.5 \mathrm{wells} / \mathrm{site}$ and 2.39 pneumatics per well [33]. Allen et al. measured 377 pneumatics at 65 production sites with in-line flow meters for a period of 15 minutes. This technique allowed the researchers to measure the gas flow both before and after the pneumatic controllers and determine how much whole gas was lost due to pneumatic operation. Of these controllers 19\% with loss rates greater than $6 \mathrm{scfh}$ accounted from $95 \%$ of emissions. More than $50 \%$ of the controllers had a loss rate less than $0.001 \mathrm{scfh}$. Sixty-seven pneumatics from wellheads were estimated to account for $1.2 \mathrm{scfh}$ on average, however, 27 measurements produced no emissions. Sites with compressors had significantly higher emissions from pneumatics at an average of 14.4 scfh [39]. Emissions from other leaks were also measured at 146 well sites and 97 leaks were measured with an average emission rate of $0.078 \mathrm{~kg} / \mathrm{hr}$ per well [39]. Luck et al. also measured emissions from pneumatic devices but focused on gathering facilities and noted that 30 of the $72(42 \%)$ behaved abnormally. The normally operating devices had an average emission rate of $0.086 \mathrm{~kg} / \mathrm{hr}$. These emissions were dominated by high-bleed devices, which according to the authors were not abnormal operators, and had an average emission rate of $0.33 \mathrm{~kg} / \mathrm{hr}$ [27].

Allen et al. and Luck et al. focused on the direct quantification of pneumatics because they were a known source of emissions. Emissions from pneumatics are often considered part of normal operation because the operation of the device necessitates that it releases a small amount of gas to maintain the pressure required. In addition to pneumatics, Allen et al. also performed emissions measurements at 150 production sites with 489 hydraulically fractured wells. The researchers measured average emissions of $1.23 \pm 0.44 \mathrm{~g}$ of methane per minute per well. Leaks were identified with a FLIR ${ }^{\circledR}$ camera and quantification measurements were made with a Hi-Flow Sampler from Bacharach [33].

The use of the Hi-Flow Sampler was a common technique to measure methane emissions directly throughout the early 2010s. The instrument was used by several researchers to quantify emissions from a variety of leaking components. However, it was later discovered that a sensor transition failure caused the device to produce erroneous measurements [43], [44]. Studies from the inventors and other researchers have presented evidence that these erroneous measurements may have resulted in underestimation of total emissions across the NG supply chain. The errors in the measurements were compared to concurrent tracerratio methods which indicated that the direct measurements from the Hi-Flow could have underestimated by 3-5 times [45]. These results may also have affected the EPA's GHGRP and emphasized the need for a more accurate method of both direct and indirect quantification methods.

Other research has focused on a variety of emissions across the supply chain. Pacsi et al. examined emissions from the entire supply chain with measurements at 67 sites. Leaks were identified with flame ionization detection (FID) and optical gas imaging (OGI) based methods. Three hundred and thirty-one leaks were discovered at 52 sites. The previously mentioned Hi-flow sampler was used for emissions 
quantification of the leaks, with the knowledge of the transition failure described. Researchers measured component level emissions from the production sector including gathering and boosting stations, however, Table 5 only presents the data from production sites [40].

Table 5. Methane emissions from components at production sites measured by Pacsi et al [40].

\begin{tabular}{|l|c|c|c|c|}
\hline \multirow{2}{*}{ Component } & \multirow{2}{*}{ Count } & \multicolumn{3}{|c|}{$\begin{array}{c}\text { Methane Emission Rate } \\
\text { (kg/hr) }\end{array}$} \\
\cline { 3 - 5 } & & Mean & Geomean & Max \\
\hline Compressors & 38 & 0.097 & 0.018 & 0.89 \\
\hline Contactor & 1 & 0.00043 & 0.00043 & 0.00043 \\
\hline Dehydrator & 2 & 0.049 & 0.0067 & 0.098 \\
\hline Discharge Piping & 1 & 0.00029 & 0.00029 & 0.00029 \\
\hline Heater Treater & 2 & 0.0044 & 0.0043 & 0.0053 \\
\hline Inlet Piping Manifold & 1 & 0.14 & 0.14 & 0.14 \\
\hline Liquid Piping Manifold Conductor & 1 & 0.0011 & 0.0011 & 0.0011 \\
\hline Meters/Piping & 27 & 0.061 & 0.007 & 1.3 \\
\hline Pig Station & 2 & 0.17 & 0.011 & 0.34 \\
\hline Separator & 38 & 0.085 & 0.007 & 1.5 \\
\hline Storage Vessel & 6 & 0.1 & 0.01 & 0.52 \\
\hline VRU Compressor & 1 & 0.14 & 0.14 & 0.14 \\
\hline Wellhead & 20 & 0.026 & 0.0032 & 0.229 \\
\hline
\end{tabular}

Kuo et al. also quantified emissions from components across the entire NG sector but focused on the state of CA. Of all the subcomponents measured only $0.4 \%$ were leaking with average rate of $2.8 \times 10^{-4} \mathrm{~kg} / \mathrm{hr}$ per component [41].The researchers utilized two methane Eagle units from RKI Instruments (Union City, CA), a Remote Methane Leak Detector (RMLD) from Heath Consultants (Houston, TX) which measured concentrations that were converted to leak rates with correlations curves and the Bacharach Hi-Flow from Health Consultants to measure flow rate directly [41].

Kang et al. used a different method of direct measurement to quantify emissions from abandoned and orphaned wells in the Marcellus region of PA. While these wells were not on active production sites, they represent the potential of wells to produce emissions. The researchers utilized a flux chamber sampling method to capture the methane emitted from the wellheads. The flux chambers were ventilated and covered the complete well head. Once the static chamber was in place for a sufficient period, a sample was pulled from the chamber for gas chromatography. The mean methane from rate at the wells was $0.27 \mathrm{~kg}$ per day per well $(\mathrm{kg} / \mathrm{d} / \mathrm{well})$. The researchers used the values of the measurements to estimate the total emission from abandoned oil and gas wells in PA to be 0.03-0.05 Mt of methane per year [42].

A similar direct quantification method to the flux chamber is known as "vacuum bagging". This type of measurement has not been used extensively in research campaigns but was previously recommended by the EPA in the early 2000s [46]. For vacuum bagging measurements, a sample of the leak is captured in an airtight bag and later sampled using an emissions analyzer or gas chromatograph. Vacuum bagging methods are time and cost intensive and are generally only applicable for small leaks.

Generally direct quantification is considered more time and cost intensive than indirect quantification methods. When applied correctly, direct methods are generally more accurate than other methods for individual measurements. Another pitfall of direct measurements is that they require direct site access and the leaks being measured must also be safely accessible. They also only provide a single measurement point in both the temporal and spatial scales. These types of measurements are often not representative of the true 
emissions profile. Methane emissions have been proven to be non-normally distributed both temporally and spatially. This fact means that single direct measurements may not be representative of the average emissions of the individual leak in question or of the type of equipment or site which acts as the leak source. When unrepresentative emissions are used to produce inventories, large errors can occur if the samples are not representative of the population.

\subsubsection{Indirect Quantification}

While direct quantification is often considered the most accurate method, it is also resource intensive. To improve the temporal and spatial understanding of emissions, indirect methods are often applied. Generally, at the cost of accuracy, these techniques paint a more general picture of emissions at site and regional levels.

\subsubsection{Other Test Method 33A and Similar Methods}

A method widely used by researchers and agencies to quantify methane emissions indirectly is Other Test Method (OTM) 33A. The EPA began development of the OTM 33 series in 2006 under the Geospatial Measurement of Air Pollution (GMAP) division. The OTM series of methods focused on three primary areas: concentration mapping, source characterization, and emissions quantification. Here, all reference to OTM will be with respect to the emission quantification portion of the method of OTM 33A. The method was designed to be used for sources that were near ground level, relatively small in source area, and within 150 meters $(\mathrm{m})$ of the measurement location [47].

In the design of the method the developers assumed that the instrumentation used for measurement would be mounted to a vehicle and because of this they defined much of the methodology with respect to road (rather than site) access. Several factors required for effective use of OTM include:

- An accessible downwind roadway from the leaking source.

- A relatively consistent wind condition blowing from the source to the point of measurement.

- Zero or few obstructions between the source and the measurement point.

- A source that is near to the ground.

- Little or no other nearby sources to the target source [47].

The method recommended multiple measurements be collected under these conditions, each ranging temporally from 15 to 20 minutes [47]. Performing multiple measurements was suggested to reduce uncertainty. However, the nature of the measurement made it difficult to obtain consistent measurements due to the reliance on uncontrollable micrometeorological variables, such as wind speed and direction. The method relies on the assumptions of an inverse dispersion modeling approach known as point source Gaussian (PSG).

The instrumentation required to complete OTM emissions quantification measurements for methane included a 3-D sonic anemometer, a concentration measurement instrument (CMI) with parts per billion ( $\mathrm{ppb}$ ) granularity, and pressure and temperature sensors. The CMI should sample as close as possible to the sonic anemometer location. The suggested setup included:

- Instrumentation on a vehicle with an extension so that the analyzers were out of the wake zone of the vehicle.

- The 180-degree $\left({ }^{\circ}\right)$ axis of the anemometer pointed toward the source that is being quantified.

- $\quad$ The emissions and wind data collected at a rate of at least $1 \mathrm{Hertz}(\mathrm{Hz})$.

- A range finder was recommended to estimate the distance from the targeted source to the measurement location.

The emission rate determined by OTM was governed by a Gaussian curve fit of average concentrations binned by wind direction. PSG methods relied on the assumption that emissions were dispersed in a pattern governed by micrometeorological variables such as wind speed and variation in wind direction. More information on the details of the calculations is presented in Section 3.2.1. 
To reduce the uncertainty of the method, a series of Method Quality Indicators (MQI) or Data Quality Indicators (DQI) were developed. A threshold on the DQI value was set to determine whether measurements could be considered reliable and accurate. There are three DQIs that were considered critical to determining the potential success of the method:

1. The fitted peak concentration bin is centered within $30^{\circ}$ of the direction of the source.

2. The average in-plume concentration is at least $0.1 \mathrm{ppm}$ above the local background

3. A gaussian fit of the concentration versus wind bin produces an $\mathrm{R}^{2}$ of greater than 0.8 [48].

Several other less critical DQIs were also defined in the final data collection methodology, source code and results [49].

Multiple studies have been conducted on the accuracy of OTM with controlled release experiments. The initial releases, performed by the EPA, took place alongside the development of the method, and were used in development of the DQIs and detailed in Brantley et al [50]. These tests consisted of 107 observations each spanning approximately 20 minutes. Releases were conducted in flat open fields in various locations. The bulk of the measurements were made with sensor and release heights of $2.7 \mathrm{~m}$ and $3.1 \mathrm{~m}$, respectively. The release rates ranged from 0.19 to 1.2 grams per second $(\mathrm{g} / \mathrm{s})$. Most of the releases $(59 \%)$ were approximately $0.6 \mathrm{~g} / \mathrm{s}$ [47]. Distances varied between $18 \mathrm{~m}$ and $179 \mathrm{~m}$ with the average distance from release to sensor being approximately $70 \mathrm{~m}$. The method produced an initial accuracy which ranged from $84 \%$ to $184 \%$. After eliminating periods of data that did not meet the primary DQIs, $74 \%$ remained. The errors of these measurements ranged from $-60 \%$ to $52 \%$, however, $71 \%$ of measurements were within $\pm 30 \%$ of the actual release rate [48].

The EPA performed field data collection using OTM soon after developing the method. Brantley et al. performed 318 measurements of 210 different production sites in three NG basins across the US. The shale basins studied were the Denver-Julesburg (DJ) in CO, the Barnett in TX, and the Pinedale in Wyoming. During data collection, the system utilized one of two closed path analyzers, either a G1301-fc cavity ringdown spectrometer or a GG-24-r off axis integrated cavity output spectrometer. The probe of the analyzer was mounted at a height of $2.7 \mathrm{~m}$ along with an $81000 \mathrm{~V}$ Ultrasonic Anemometer. The DAQ setup also included a compact weather station and a global positioning system (GPS). The 318 measurement distances ranged from 10 to $200 \mathrm{~m}$ with an average distance of $57 \mathrm{~m}$. Measurement durations ranged from 15-20 minutes. Only well pads that produced a downwind methane enhancement of greater than $0.1 \mathrm{ppm}$ were measured. Several sites in the Pinedale Basin were measured multiple times to improve the understanding of the OTM technique and the variability of the source emissions. Complete details of the repeat measurements were presented in the SI and are presented in Table 6 [50]. The mean and confidence levels (CL) were calculated using non-parametric bootstrapping. 
Table 6. Results and statistics of repeated methane measurements by Brantley et al. [50]

\begin{tabular}{|c|c|c|c|c|c|c|}
\hline \multirow{2}{*}{ Site } & $\mathbf{N}$ & Mean & $\begin{array}{c}\text { Lower } \\
\mathbf{C L}\end{array}$ & $\begin{array}{c}\text { Upper } \\
\mathbf{C L}\end{array}$ & $\begin{array}{c}\text { Minimum } \\
\text { time } \\
\text { between } \\
\text { samples }\end{array}$ & $\begin{array}{c}\text { Maximum } \\
\text { time } \\
\text { between } \\
\text { samples }\end{array}$ \\
\cline { 2 - 7 } & $\mathbf{( \# )}$ & $\mathbf{( g / s )}$ & $\mathbf{( g / s )}$ & $\mathbf{( g / s )}$ & (days) & (days) \\
\hline A & 3 & 0.04 & 0.02 & 0.05 & 0 & 4 \\
\hline B & 4 & 0.07 & 0.06 & 0.08 & 0 & 732 \\
\hline C & 21 & 0.16 & 0.12 & 0.2 & 0 & 378 \\
\hline D & 4 & 0.2 & 0.08 & 0.36 & 2 & 388 \\
\hline E & 6 & 0.44 & 0.25 & 0.71 & 0 & 6 \\
\hline F & 3 & 0.9 & 0.45 & 1.3 & 0 & 3 \\
\hline G & 3 & 1.1 & 0.65 & 1.46 & 0 & 0 \\
\hline H & 6 & 0.71 & 0.2 & 2.59 & 0 & 336 \\
\hline I & 10 & 1.95 & 1.16 & 3.52 & 0 & 5 \\
\hline
\end{tabular}

The repeat measurements performed by this study lend credence to three possible scenarios. Those scenarios are that emissions from production sites were episodic in nature and inconsistent, that the method used to measure the said emissions has high uncertainty and produced inconsistent results, or a combination of the two. It is well documented that methane emissions from the natural gas infrastructure have a high temporal variance [28], [29]. However, even when compared to known emissions rates the OTM method produced errors ranging from $-70 \%$ to $170 \%$. This suggests that the third option was in play and that one should not place much confidence in indirect measurements with high uncertainty made over a short duration (15-20 minutes).

Some measurements from the initial study of OTM took place during concurrent direct measurements made by Eastern Research Group (ERG) and Allen et al [33]. The mean emissions measured using OTM in the Barnett shale were more than double the mean measured by direct measurements $(0.33 \mathrm{~g} / \mathrm{s}$ compared to $0.14 \mathrm{~g} / \mathrm{s})$. The authors acknowledged this difference but noted that the interquartile ranges of the direct and OTM measurements overlapped. The authors also noted that the OTM emissions were biased towards high emitters and noted that the detection limit of OTM was about $0.01 \mathrm{~g} / \mathrm{s}$ [50]. A summary of measurements made during the study is presented in Table 7. Confidence intervals (CI) were determined with a nonparametric bootstrap. The $95 \%$ CIs were between $69 \%$ and $145 \%$ of the geometric mean, generally weighted toward overestimation.

Table 7. OTM methane emission measurements and results by basin as reported by Brantley et al [50].

\begin{tabular}{|c|c|c|c|c|c|c|}
\hline Basin & Year & Measurements & $\begin{array}{c}\text { Average } \\
\text { Distance } \\
\text { [m] } \\
\text { (Range) }\end{array}$ & $\begin{array}{l}\text { Percent } \\
\text { Invalid }\end{array}$ & $\begin{array}{c}\text { Median } \\
\text { Rate } \\
{[\mathrm{kg} / \mathrm{hr}]}\end{array}$ & 95\% CI [kg/hr] \\
\hline Barnett & \multirow{3}{*}{$\begin{array}{c}2010- \\
2013\end{array}$} & 43 & \multirow{3}{*}{$\begin{array}{c}57(10- \\
200)\end{array}$} & \multirow{3}{*}{$29 \%$} & 1.19 & $0.83-1.73$ \\
\hline DJ & & 74 & & & 0.5 & $0.40-0.68$ \\
\hline Pinedale & & 107 & & & 2.12 & $1.69-2.66$ \\
\hline
\end{tabular}

Another team from the University of Wyoming has used a mobile laboratory to conduct numerous studies involving OTM [51]-[54]. Their DAQ setup included a G2204 Cavity Ringdown Spectrometer from 
Picarro, Inc. and an R.M. Young 81000 ultrasonic anemometer, as well as an all-in-one weather station and a GPS. Initial data collection using this setup included both controlled release measurements and field data collection [51], [54].

Robertson et al. performed a second series of controlled release experiments at Christman Airfield (CAF) in Fort Collins, $\mathrm{CO}$ for comparison with those performed by Brantley et al [50], [51]. Snare also provided further analysis on the 23 controlled releases performed by Robertson et al. in a master's thesis [54]. He made several notes on optimal conditions for the method based on these controlled releases including:

- Optimal distance is less than $100 \mathrm{~m}$ unless there are high wind speeds and atmospheric stability

- Average wind speeds should be greater than $1 \mathrm{~m} / \mathrm{s}$

- Accurate flux measurements can be obtained in wind speeds up to $10 \mathrm{~m} / \mathrm{s}$

This series of experiments consisted of 23 tests. Nineteen of the tests were manifold releases and four were point source releases. The manifold releases were made from a $3 \mathrm{~m}$ long tube with $3 \mathrm{~cm}$ holes drilled every $10 \mathrm{~cm}$. Four of the measurements were eliminated from analysis because they did not pass one of the three primary DQIs. A point source test was eliminated due to high wind variance and 3 manifold releases were eliminated due to low Gaussian fits. Tests with flags for low methane levels (8) were included in the analysis. These tests produced relatively low errors, leading Snare to suggest that the flag was too conservative. Low methane level flags typically occurred in releases below $7.5 \mathrm{lpm}(\sim 0.09 \mathrm{~g} / \mathrm{s})$. So, 19 tests were included in the final analysis with release rates ranging from 0.11 to $2.02 \mathrm{~kg} / \mathrm{hr}$ and distances between 30 and $175 \mathrm{~m}$. The average error was $-0.6 \pm 27 \%$. Another note was that meteorology during the controlled releases displayed little variance. All tests had an Atmospheric Stability Indicator (ASI) from 4-6. This value, which can range from 1-7, acted as a measure of atmospheric stability. During the tests, wind speeds were between 2 and $10 \mathrm{~m} / \mathrm{s}$ [51]. Robertson et al. combined these results with those from the Brantley et al, which resulted in a dataset of 119 tests. These data had a $2 \sigma$ error of $\pm 56 \%$ and a $1 \sigma$ error of $\pm 28 \%$. Robertson et al. reported a 10\% low bias but noted that the Brantley et al. data did not share this bias [51].

During their field campaign Robertson et al. used OTM methods to estimate emissions from well pads in four natural gas basins. The field data collected consisted of 218 measurements of well pads from four different shale plays. The study analyzed an additional 68 measurements performed Brantley et al [50]. The study calculated $95 \%$ CIs with parametric bootstrapping and determined that they ranged between $32 \%$ and $246 \%$ of the median. Table 8 contains information from the field campaign of Robertson et al [51].

Table 8. OTM methane emission measurements and results by basin reported by Robertson et al [51].

\begin{tabular}{|c|c|c|c|c|c|c|}
\hline Basin & Year & Measurements & $\begin{array}{c}\text { Average } \\
\text { Distance [m] } \\
( \pm \mathbf{1 \sigma})\end{array}$ & $\begin{array}{l}\text { Percent } \\
\text { Invalid }\end{array}$ & $\begin{array}{c}\text { Median } \\
\text { Rate } \\
{[\mathrm{kg} / \mathrm{hr}]}\end{array}$ & $\begin{array}{c}95 \% \text { CI } \\
{[\mathrm{kg} / \mathrm{hr}]}\end{array}$ \\
\hline $\begin{array}{c}\text { Upper Green } \\
\text { River } \\
\end{array}$ & $2014 / 15$ & 51 & $84(43)$ & $30 \%$ & 2.3 & $1.7-3.1$ \\
\hline DJ & 2014 & $84 *$ & $75(40)$ & $33 \%$ & 1.4 & $0.86-2.3$ \\
\hline Uintah & 2015 & 30 & $112(45)$ & $30 \%$ & $\begin{array}{l}.7 \text { (gas) } \\
1.9 \text { (oil) }\end{array}$ & $\begin{array}{l}1.2-9.1 \text { (gas) } \\
0.79-3.5 \text { (oil) }\end{array}$ \\
\hline Fayetteville & 2015 & 53 & $46(24)$ & $24 \%$ & 0.68 & $0.40-1.1$ \\
\hline
\end{tabular}

Post analysis of the measurements focused on comparison to gas and oil throughput as well as water produced by the sites measured. Throughput normalized mass emissions were determined by dividing the mass emission rate measured by the amount of methane in the natural gas produced. They determined that for natural gas wells this value ranged from $0.09 \%$ for sites with no manual unloading of wells (in the Fayetteville shale) to $2.8 \%$ in the Uintah shale region. They emphasized a common trend in natural gas emissions measurements in which $20 \%$ of the highest emitters in each region accounted for $72-83 \%$ of all 
methane emissions. This skewed distribution has been demonstrated across a range of natural gas emissions studies which emphasize "high-emitters" or "super-emitters".

Snare delved deeper into the measurements made in the Upper Green River Basin and compared them to aircraft-based emissions estimates. The data collection campaign describes two deployments, one consisting of 27 plume measurements and another consisting of 36 measurements. Snare estimated that 275 oil and gas wells were represented by these 63 measurements. Eighteen measurements were removed for high DQI values. Of the 45 measurements remaining 24 were of single-well pads, 21 were of multi-well pads, and one was a repeated measurement. Geometric mean emissions rates were $0.34 \mathrm{~g} / \mathrm{s}$ (95\% CI: 0.2$0.48 \mathrm{~g} / \mathrm{s}$, median value: $0.33 \mathrm{~g} / \mathrm{s}$ ) [54].

Bell et al. compared 50 OTM field measurements from the Robertson et al [51], [55] Fayetteville campaign to concurrent tracer ( 7 facilities) and direct measurements (43 facilities). This study reported overlapping 95\% CIs between the OTM and direct measurements at 28 of the 43 facilities. However, they also reported that variance weighted least-squares (VWLS) regressions indicated statistically significant differences between the methods. The 43 sites were subdivided into three groups.

- $\quad$ Sites with no detectable emissions based on downwind transects (10)

- $\quad$ Sites with liquid unloadings included in the direct measurements (5)

- $\quad$ Other sites (28)

The differences between the OTM measurements and on-site direct measurements were dominated by the sites with liquid unloadings. A complete breakdown of the comparison between OTM facility estimates (OFE) and study onsite estimates (SOE) from the authors and is presented in Figure 7.

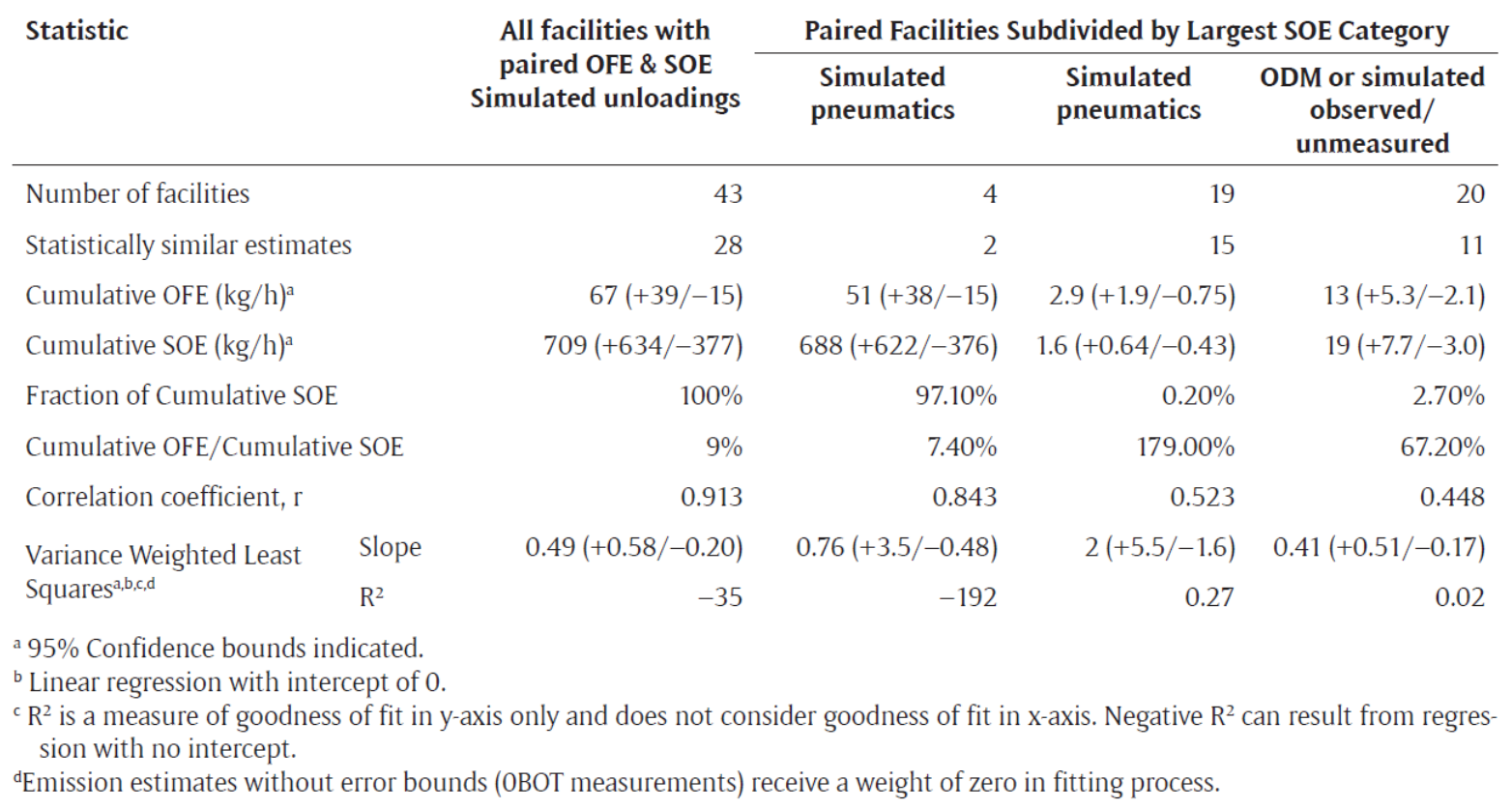

Figure 7. Comparison by Bell et al. of OTM and direct site measurement results [55].

A recent study by Edie et al. further investigated the controlled release experiments of Robertson et al., and performed a third set of controlled releases at the Methane Emissions Technology Evaluation Center (METEC), also in Fort Collins. Table 9 presents a comparison of these two release test sets [53].

The CAF data set focused on the effectiveness of OTM in an open area with a single known source location, whereas the METEC set was conducted with the potential for multiple leak sources from different 
components at a mock NG production site. The study observed that across each test set the wind speed, number of sources, and leak height had no major impact on estimate accuracy.

Table 9. OTM controlled release results as analyzed by Edie et al [53].

\begin{tabular}{|l|c|c|}
\hline Author & Robertson et al [51]. & Edie et al [53]. \\
\hline Year & 2014 & 2017 \\
\hline Location & CAF & METEC \\
\hline Description & $\begin{array}{c}\text { Open flat field with } \\
\text { single source }\end{array}$ & $\begin{array}{c}\text { Mock natural gas } \\
\text { site with multiple } \\
\text { sources }\end{array}$ \\
\hline Number of Tests & 23 & 34 \\
\hline Number Passing DQI & 19 & 24 \\
\hline Release Rates (kg/hr) & $0.11-2.02$ & $0.14-2.16$ \\
\hline Distances (m) & $34-174$ & Varied (multiple) \\
\hline Wind Speeds (m/s) & $2-8$ & $2-9$ \\
\hline PGIs & $2-6$ & $3-6$ \\
\hline Total Error Range (\%) & $-75 \%$ to $50 \%$ & $-60 \%$ to $+170 \%$ \\
\hline $\mathbf{6 8}^{\text {th }}$ Percentile Error & $\pm 28 \%$ & $\pm 38 \%$ \\
\hline Tests within $\pm \mathbf{5 0 \%}$ & $85 \%$ & $85 \%$ \\
\hline
\end{tabular}

Several regression techniques were used to analyze the data. Ordinary least squares (OLS) regression resulted in OTM underestimating by $8 \%$. To minimize the influence of large release rates on OLS results, both orthogonal distance regression and VWLS regression were applied. Applying a measurement uncertainty of $\pm 50 \%$ to the data and setting the intercept to zero results in slopes of 0.79 and 0.67 for these two methods. These results suggest that OTM estimates were $20-33 \%$ lower than known releases. An OLS regression analysis confirmed the $10 \%$ low bias seen by Robertson et al. and a propensity to overestimate smaller releases. The study concluded that OTM measurements could generally expect a $2 \sigma$ error of $\pm 70 \%$ with a slight negative bias. These various studies comparing OTM to controlled release experiments have consistently found uncertainties greater than $\pm 50 \%$. The study suggested that OTM estimates were significantly influenced by source distance. A 5\% change in distance results in a $10 \%$ increase in estimate. Using an average source distance when combining measurements from multiple sources had a minimal error contribution relative to the overall error of $\pm 70 \%$. The study suggested that no significant trends existed between estimate error and wind speed, number of sources or difference between source and sample heights [53].

The latest field campaign was another by Robertson et al. in New Mexico's (NM) Permian basin. The study consisted of 111 site-level measurements, 71 of which were successful estimates from oil and gas production well pads. The measurements were further subdivided by state and site complexity. A simple site was defined as one with little or no liquid storage and processing equipment on-site. Complex sites were those with one or more oil or water storage tanks and or compressors. The 71 successful estimates consisted of: 46 sites in NM (30 complex and 16 simple) and 25 sites in TX (17 complex, 8 simple). Seventeen (13 simple, 4 complex) of the NM sites were below the detectable limit (BDL) of $0.01 \mathrm{~g} / \mathrm{s}$ defined by Brantley et al. Twelve ( 6 complex, 6 simple) of the TX sites were BDL. The lowest quantified rate during the field campaign was $0.068 \mathrm{~kg} / \mathrm{hr}[52]$. 
In this study the same bootstrapping method as was used in Robertson et al (2017) was again utilized [51]. The error distribution of the bootstrap utilized the $1 \sigma$ error of $(+54 \% /-26 \%)$ as the standard deviation and were resampled with replacement 100,000 times creating 100,000 sample sets with 71 estimates each. These results were used to determine $95 \%$ CI. Estimates of mean mass emissions rates from the study are presented in Table 10.

Table 10. OTM methane emission measurements, as reported by Robertson et al. 2020 [52].

\begin{tabular}{|c|c|c|c|c|c|c|c|c|}
\hline \multirow{2}{*}{ Dataset } & \multicolumn{4}{|c|}{ Total } & \multicolumn{3}{c|}{$\begin{array}{c}\text { Below Detectable Limit } \\
\text { (BDL) }\end{array}$} & \multicolumn{3}{c|}{ Both } \\
\cline { 2 - 9 } & $\#$ & Simple & Complex & $\#$ & Simple & Complex & $\begin{array}{c}\text { Mean Mass } \\
\text { Emissions (kg/hr) }\end{array}$ & $\mathbf{9 5 \% ~ C I ~}$ \\
\hline NM & 46 & 30 & 16 & 17 & 13 & 4 & 4.74 & $2.58-7.61$ \\
\hline TX & 25 & 8 & 17 & 12 & 6 & 6 & 1.89 & $0.70-3.52$ \\
\hline Both & 71 & 38 & 33 & 29 & 19 & 10 & 3.76 & $2.24-5.71$ \\
\hline
\end{tabular}

The study also emphasized the trend of "super-emitters" stating that the $15 \%$ of emitters had emissions rates greater than $7 \mathrm{~kg} / \mathrm{hr}$ and accounted for over $70 \%$ of total basin emissions. The top $5 \%$ of emitters had emissions of at least $20 \mathrm{~kg} / \mathrm{hr}$ and accounted for over $30 \%$ of total emissions [52].

Even with these large uncertainties, data from collected from the field continues to be used for comparisons with regional and national emissions estimated. Several studies have used OTM to estimate emissions from active production sites. This produces high levels of uncertainty in the reporting of emissions from these studies. A few studies have utilized data acquisition setups like those used in OTM while utilizing modified measurement techniques. These studies have focused on the measurement of downwind methane flux but have quantified the results with different methods.

Albertson et al. utilized the same data acquisition setup as Brantley et al [50], [56]. However, their method focused on mobile "passes" rather than stationary measurements. The researchers utilized multiple passes downwind of the targeted source with the goal of transecting the flux (i.e., the resulting downwind plume). Much of the research focused on path planning and optimal route selection for quantification of as many pads in a region as possible with minimal interference. While this was necessary for regional estimates, it was not required for individual sources estimations. The researchers performed one controlled release test in Durham, North Carolina which was a point source with a rate of $0.6 \mathrm{~g} / \mathrm{s}$ and a height of $1.5 \mathrm{~m}$. They attempted to quantify this source with three downwind transects. The novelty of the method used was the employment of Bayesian posterior distribution. This allowed researchers to make two estimates with each pass, one being the inferred release rate with fixed prior and the second with a recursively updated prior. The recursively updated prior allowed for information from previous passes be utilized in estimating the current pass emission rate. This knowledge improved their estimate after three passes of the controlled release. The estimates with these two methods are presented in Table 11. The researchers suggest that the method of recursive updating leads to more accurate leak rate estimation and reduced uncertainty with an increased number of sensor passes. Field data were collected in CO in July of 2010, with the number of passes for each quantification attempt ranging in number between two and five. The results of the field data are presented in Table 12. This study lends credence to the fact that repeat measurements are critical for reduced uncertainty. The researchers also evaluated the sensitivity of the method to height of the source and estimated distance from the source. They estimate that a $10 \%$ error in source height has less than a $3 \%$ impact on the estimation and a $50 \%$ error in longitudinal distance only had a $6 \%$ impact on the estimate. These impacts were far less than those from OTM studies with single measurements, which were much more sensitive to source distance estimates. 
Table 11. Controlled methane release results using mobile passes, as reported by Albertson et al. [56].

\begin{tabular}{|c|c|c|c|}
\hline Pass \# & $\begin{array}{c}\text { Release } \\
\text { Rate }(\mathbf{g} / \mathbf{s})\end{array}$ & $\begin{array}{c}\text { Estimate w/ fixed } \\
\text { prior } \mathbf{( g / s )}\end{array}$ & $\begin{array}{c}\text { Estimate w/ updated } \\
\text { prior }(\mathbf{g} / \mathbf{s})\end{array}$ \\
\hline 1 & $0.6 \pm 0.03$ & 0.65 & 0.65 \\
\hline 2 & $0.6 \pm 0.03$ & 0.27 & 0.51 \\
\hline 3 & $0.6 \pm 0.03$ & 0.73 & 0.60 \\
\hline
\end{tabular}

Table 12. Field measurement results using mobile passes, as reported by Albertson et al [56].

\begin{tabular}{|c|c|c|c|}
\hline Study \# & \# Passes & $\begin{array}{c}\text { Average Estimate w/ } \\
\text { fixed prior }(\mathbf{g} / \mathbf{s})\end{array}$ & $\begin{array}{c}\text { Estimate w/ updated } \\
\text { prior }(\mathbf{g} / \mathbf{s})\end{array}$ \\
\hline 1 & 5 & 1.8 & 2.1 \\
\hline 2 & 3 & 0.8 & 0.8 \\
\hline 3 & 2 & 1.6 & 1.4 \\
\hline 4 & 3 & 0.18 & 0.16 \\
\hline
\end{tabular}

Zhou et al. utilized the same methodology as Albertson et al. to quantify methane emissions from NG production sites in CA and against a series of controlled release experiments [56], [57]. The methodology applied during this research required a 3-D sonic anemometer mounted on a vehicle along with a methane concentration analyzer, in this case an open-path LI-7700 from LICOR $\AA$. The vehicle then made downwind traverses while a stationary tower was deployed near the site with a second 3-D sonic anemometer for the purpose of measuring micrometeorological variables. Twenty-two controlled release experiments were performed with methane flow rates varying from 5 to $25 \mathrm{slpm}$, release heights from 0.15 to $3 \mathrm{~m}$, and mean distances from 40.6 to $80.3 \mathrm{~m}$. Like Albertson et al. the researchers made multiple downwind passes and updated estimates using a Gaussian likelihood function. The controlled release rate was within the uncertainty bounds of the estimate $81 \%$ of the time with a mean error of $8 \%$. The lower limit of detection was determined to be $4 \mathrm{~kg} /$ day $(166 \mathrm{~g} / \mathrm{hr})$ for distances less than $100 \mathrm{~m}$. A two-week field campaign was conducted by the researchers in CA in which they measured 87 (66 active) well pads. The measurement distances ranged from 10 to $160 \mathrm{~m}$ with a mean distance of $25 \mathrm{~m}$. The active well pads had emissions rates ranging from 0.04 to $230 \mathrm{~kg}$ /day. The estimated mean emission rate of these well pads was $9.5 \mathrm{~kg} / \mathrm{day}(95 \%$ CI: 6.1-14.6). The data were highly skewed with the top $10 \%$ and $20 \%$ of emitters contributing $80 \%$ and $90 \%$ of emissions, respectively. The authors also stated that "one of the limitations of this study is the relatively short duration of measurements, which limits the ability to capture temporal dynamics of emission or any seasonality from surveyed sources" [57].

Yacovitch et al. used a mobile laboratory combined with known tracer releases to estimate emissions from a variety of NG facilities in the Barnett shale region. The technique relied on concentration measurements of the tracer gas (in this case $\mathrm{CO}_{2}$ ) and the gas being quantified (methane). The method utilized a forward Gaussian dispersion simulation and many of the assumptions required by the method were the same as those for OTM. To verify the method a series a 5-day dataset of controlled tracer releases were performed with 2-5 tracer gases. Using this dataset, a 95\% CI of $0.334 x-3.34 x$ was assigned to the tracer method, for an emission of size $x$. OTM was compared using the staged release data and $95 \%$ CI was determined to be $0.4 x$ to $1.6 x$. Field measurements were taken using tracer releases of $\mathrm{CO}_{2}$ at specific sites. The production site results from this campaign are presented in Table 13 [58].

Yacovitch et al. performed a second series of tracer flux measurements in two other US NG basins, the DJ and Fayetteville. During this campaign tracer releases of nitrous oxide or ethyne were used along with a mobile laboratory that performed downwind transects. The flow of methane from the site was estimated 
using the known flow rate of the tracer and a ratio of the methane to tracer concentration enhancement. In this case the researchers did not rely at all on dispersions assumptions that are required for other downwind techniques. The study determined that the mode of measurements from production sites in the Fayetteville basin was $1.0 \mathrm{~kg} / \mathrm{hr}(95 \% \mathrm{CI}$ : $0.36-12, \mathrm{n}=10)$. They noted one "super-emitter" which had a rate of 802 $\mathrm{kg} / \mathrm{hr}$, an example of the phenomenon of temporal or fat-tailed distributions of methane emissions from NG production sites. All measurements of production sites from the study are summarized in Table 13 [58].

Caulton et al., Goetz et al., and Omara et al. (2016) all performed tracer flux measurements of production sites in the Marcellus shale region [19], [35], [59]. Caulton et al. had the largest dataset of these studies consisting of 2100 measurements of 673 unconventional well pads. The measurements had log-normal distribution with a geometric mean of $2.0 \mathrm{~kg} / \mathrm{hr}$ and an arithmetic mean of $5.5 \mathrm{~kg} / \mathrm{hr}$. The measurements that were unique to well pads had a geometric mean of $1.7 \mathrm{~kg} / \mathrm{hr}$ and an arithmetic mean of $4.3 \mathrm{~kg} / \mathrm{hr}$. They determined that their limit of detection was $0.12 \mathrm{~kg} / \mathrm{hr}$. The emissions measurements made during this study displayed the continuing trend of "super-emitters" with the top $10 \%$ of emitters contributing $73 \%$ of emissions and the top $5 \%$ contributing $61 \%$ of emissions. They defined "super-emitters" as similarly to Zavala-Araiza et al., but estimated that the top $1 \%$ of sites contributed an average of $9 \mathrm{~kg} / \mathrm{hr}$ [35]. Omara et al. measured 18 conventional production sites with 19 wells and 17 unconventional sites with 88 wells. The mean pad-level emission rate from unconventional sites ( $18.8 \mathrm{~kg} / \mathrm{hr}, 95 \% \mathrm{CI}: 12.0-26.8 \mathrm{~kg} / \mathrm{hr}$ ) was 23 times that of the pad-level emissions from conventional sites. The study was another example of fat-tailed distributions from unconventional sites. Of the 13 measured sites with no flowback the three highest emitters contributed $85 \%$ of emissions from unconventional sites. This study extrapolated their emissions estimates to regional estimates and projected that the total annual emissions from 88,500 conventional and 3390 unconventional wells in the larger PA-WV region were 1,150 Gigagrams [26]. Goetz et al. mostly focused on compressor sites but did measure three production sites with a tracer flux method [59]. Summary data from these studies are presented in Table 13.

The primary hurdles to using tracer release methods are resources and access. They typically require two coordinated teams, one performing the releases and the other measuring the downwind plumes. Release methods also require that the tracer gas be released reasonably close to the expected source, atmospheric conditions for good transport and that the downwind area is accessible by vehicle. These drawbacks make it difficult to use in the field as it is time and resource intensive.

Rella et al. performed downwind transects with a mast containing multiple sampling ports at different heights attached to the vehicle [60]. The samples drawn from these ports were stored in AirCores, which are long thin coils for storing samples to be analyzed later. The theory of the AirCore principle is that the gas does not sufficiently diffuse within the core and therefore when it is pumped out of the core and into a CMI it gives the researcher a time series of concentration measurements [61]. The researchers claimed that using multiple sampling ports with the AirCores allowed them to overcome the single point limitations of other downwind flux techniques. Multiple sample points during the downwind transect allowed researchers to better map the plume of study. The samples stored in the AirCores were later analyzed with a Picarro G2301. Wind speed and direction were also measured with a sonic anemometer, like those used in other studies. In a series of controlled releases with distances ranging from $5 \mathrm{~m}$ to $81 \mathrm{~m}$ the accuracy of this method was determined to be $24 \%$ with $67 \%$ of measurements within 1.9 times the geometric mean. The detection limit was determined to be $0.034 \mathrm{~kg} / \mathrm{hr}$. The controlled releases captured a wide range of atmospheric conditions, distances, and release rates. As a result of these experiments, researchers defined requirements for valid field as:

- $\quad$ wind speed greater than $1 \mathrm{~m} / \mathrm{s}$

- measurements clearly attributed to a single well pad

- estimated distance of $150 \mathrm{~m}$ or less

- a centroid of the plume below the height of $4.2 \mathrm{~m}$

- a width of the plume less than $5 \mathrm{~m}$ 
Field measurements were taken over 16 days in the Barnett shale resulting in 207 flux measurements. Of the 207 measurements $45 \%$ did not meet the defined criteria outlined above. The 115 flux measurements that were included are presented in Table 13. The researchers noted a log-normal distribution with $20 \%$ of emissions from the top $1.1 \%$ of emitters and $80 \%$ of emissions from the top $20 \%$ of emitters. Through mapping and subsequent modeling, the researchers estimated that 193 well pads were covered by the downwind transects. Of these, 71 had no measurable signal, which indicated BDL emissions. After bootstrapping and a Monte Carlo analysis they estimated emissions distributions from well pads to have the following statistics.

- Geometric mean of $0.72 \pm 0.11 \mathrm{~kg} / \mathrm{hr}$

- $\quad$ Arithmetic mean of $1.63 \pm 0.33 \mathrm{~kg} / \mathrm{hr}$ [95\% CI: $1.09-2.38]$

Lan et al. used a mobile laboratory outfitted with two Picarro cavity ringdown instruments [62]. To estimate emissions, they used a simple Gaussian Dispersion model (GDM). A Monte Carlo probabilistic uncertainty method was used to estimate 95\% CIs. They also applied EPA's AERMOD modeling simulation software for seven specific sites, however, none of these were well pads. Emissions rates of well pads measured ranged from 0.0009 to $58.0 \mathrm{~kg} / \mathrm{hr}$ and displayed a fat-tailed distribution with $70 \%$ of pads having an emission rate less than $5 \mathrm{~kg} / \mathrm{hr}$. The calculated uncertainties of the well pad measurements range from -28\% to $81 \%$. They found a statistically significant correlation between production rate and pad emissions and used a linear relationship to estimate total well pad emissions in the Barnett region to be $1.5 \times 10^{5} \mathrm{~kg} / \mathrm{hr}$ (uncertainty range $1.16-2.04 \times 10^{3} \mathrm{~kg} / \mathrm{hr}$ ) [62].

Saide et al. attempted to reduce the spread of results from OTM estimates by combining calculations with Large-eddy simulations (LES) [63]. The simulations utilized two hours of emissions representative of actual well pad emission from seven modeled well pads each containing two wellheads. The modeling method utilized the OTM sampling strategy but used a stochastic sampling method and Bayesian interface to estimate emissions from the pad. The method was able to reduce the spread of emissions compared to OTM alone and resulted in $92-95 \%$ of estimations within $30 \%$ of the actual rate. While these methods may increase accuracy, they are time and computationally expensive. Simulation of one hour's worth of emissions required approximately 24 hours with 1,296 processors on a high-performance computing platform. In order to be used effectively in the field the method would also require a large number of different simulations for differing pads and micrometeorological conditions [63]. 
Table 13. Indirect methane measurement campaigns and results for production sites (kg/hr).

\begin{tabular}{|c|c|c|c|c|c|c|c|c|c|}
\hline Lead Author & $\begin{array}{c}\text { Published } \\
\text { Year }\end{array}$ & Technique & $\begin{array}{c}\# \\
\text { Sites }\end{array}$ & Region / State & $\begin{array}{c}\text { Geometric } \\
\text { Mean }\end{array}$ & Mean & Median & Min & $\operatorname{Max}$ \\
\hline Goetz & 2015 & Tracer & 3 & Marcellus & 7.5 & 8.8 & & 3.4 & 14.2 \\
\hline Caulton & 2019 & $\begin{array}{c}\text { Mobile } \\
(\text { Transects) })^{\mathrm{a}}\end{array}$ & 673 & Marcellus & 1.7 & 4.3 & & & \\
\hline Omara & 2016 & Tracer & $17^{\mathrm{b}}$ & Marcellus & & 18.8 & & 0.9 & 92.9 \\
\hline \multirow{2}{*}{ Yacovitch } & \multirow{2}{*}{2017} & \multirow{2}{*}{ Tracer } & \multirow{2}{*}{23} & $\mathrm{DJ}^{\mathrm{c}}$ & 17.3 & 33.3 & & 2.3 & 74.0 \\
\hline & & & & Fayetteville & 1.4 & 48.4 & & 0.0 & 802.0 \\
\hline \multirow{4}{*}{ Robertson } & \multirow{4}{*}{2017} & \multirow{4}{*}{ Mobile (OTM) } & \multirow{4}{*}{51} & Uintah & & & 3.7 & & \\
\hline & & & & $\mathrm{DJ}$ & & & 1.4 & & \\
\hline & & & & Upper Green River & & & 2.3 & & \\
\hline & & & & Fayetteville & & & 0.7 & & \\
\hline Albertson $^{\mathrm{d}}$ & 2016 & Mobile (PSG) & 4 & Colorado & 2.8 & 4.0 & & 0.6 & 7.6 \\
\hline \multirow{3}{*}{ Brantley } & \multirow{3}{*}{2014} & \multirow{3}{*}{ Mobile (OTM) } & \multirow{3}{*}{$210^{\mathrm{e}}$} & Barnett & 1.2 & & & & \\
\hline & & & & DJ & 0.5 & & & & \\
\hline & & & & Pinedale & 2.1 & & & & \\
\hline Lan & 2015 & Mobile (PSG) & 34 & Barnett & & & & 0.0 & 58.0 \\
\hline Rella & 2015 & Mobile (Flux) & $115^{\mathrm{f}}$ & Barnett & 0.6 & 1.7 & & 0.027 & 47.6 \\
\hline Yacovitch & 2015 & Tracer (Flux) & 46 & Barnett & 7.2 & 28.3 & & 0.1 & 259.4 \\
\hline Zhou & 2021 & Mobile (PSG) & 87 & California & & 0.4 & & 0 & 9.58 \\
\hline
\end{tabular}
a) Median limit of detection $0.12 \mathrm{~kg} / \mathrm{hr}$
b) Unconventional sites
c) Targeted high emitters
d) Result of final pass using Bayesian inference with recursively updated prior
e) Accepted sites $>0.036 \mathrm{~kg} / \mathrm{hr}$
f) 67 sites also measured with detected emissions 


\subsubsection{The Eddy Covariance Flux Method}

Another way to measure gas flux is the Eddy Covariance (EC) method. EC is a technique generally used to measure a vertical flux in the atmosphere. The flux is the amount of the substance being measured that passes through a defined space in a defined period of time. The flux being measured could be heat, water, or any other gas, including methane. EC is one of the most direct and defensible ways to measure these fluxes [64]. The principles described here generally apply to any flux measurement, however, for the purpose of this research methane will be considered the flux measurement in question. EC is generally applied to calculate vertical fluxes within the atmospheric boundary layer between the atmosphere and different ecosystems [64]. Typically, EC measurements have been applied to a flat, homogenous area sources. The calculations involved in EC are complex, however, they are based on first principles. It has gained popularity over the last decade due to improvements in the instrument technology required to perform accurate measurements. A number of networks have arisen that track fluxes of various gases around the world, and as such, terminology and methodology are trending towards uniformity, however, there are still a number of differences in how EC data post processing is performed.

The general theory of EC has been outlined in detail by Burba et al [64]. The simplest description is the measurement of a vertical flux. The principles are based on the nature of air flow within the atmospheric boundary layer. While the bulk fluid motion of the atmosphere is typically horizontal in the form of wind, the turbulent nature of the motion also creates "eddies" which form within that horizontal motion. These eddies are made of circular motions within the bulk flow and thus have both vertical and horizontal components. The components of interest when measuring fluxes are the vertical variations. Figure 8 presents a high-level representation of this type of motion [65]. Using high frequency instruments to measure vertical wind speeds and methane concentrations, a net transfer of the plane can be determined at any given time. The change in these measurements is the vertical methane flux.

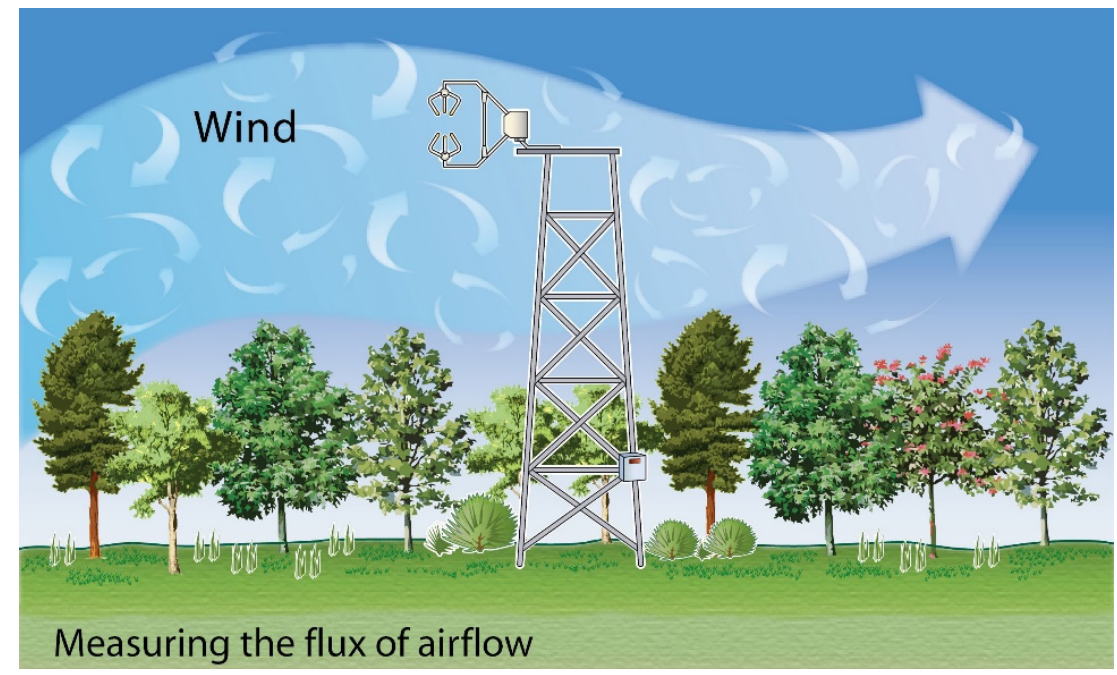

Figure 8. General representation of an EC application [65].

The mathematical theory of EC is that the vertical flux can be represented as the covariance between the vertical velocity and the concentration methane [64]. These values are determined with the use of Reynolds averaging, which is a common technique in the evaluation of turbulent flows. Reynold's averaging breaks down measurements over time into means and deviations. When applied to EC equations, Reynold's averaging allows for many terms in a complex equation to be removed because they are "average deviations from the average" or zero. Additional assumptions are made based on the nature of the air (primarily that fluctuations in air density are assumed to be zero and that the mean vertical flow is zero). This simplifies the equation to the final "Eddy Flux". Equation 1 through Equation 7 presented below, were defined by Burba et al. as a derivation and represent the mathematical basis of these simplifications [64]. 


$$
F=\overline{\rho_{d} W S}
$$

Where,

- $\quad \rho_{\mathrm{d}}$ is the air density

- $\quad w$ is the vertical wind speed

- $\quad s$ is the dry mole fraction of the gas of interest in air

Applying Reynold's decomposition breaks the terms into averages $(\bar{x})$ and deviations from the average $\left(x^{\prime}\right)$ which yields:

$$
F=\overline{\left(\overline{\rho_{d}}+\rho_{d}{ }^{\prime}\right)\left(\bar{w}+w^{\prime}\right)\left(\bar{s}+s^{\prime}\right)}
$$

Equation 2

Expanding the equation:

$$
=\overline{\left(\overline{\rho_{d}} \bar{w} \bar{s}+\overline{\rho_{d}} \bar{w} s^{\prime}+\overline{\rho_{d}} w^{\prime} \bar{s}+\overline{\rho_{d}} w^{\prime} s^{\prime}+\rho_{d}{ }^{\prime} \bar{w} \bar{s}+\rho_{d}{ }^{\prime} \bar{w} s^{\prime}+\rho_{d}{ }^{\prime} w^{\prime} \bar{s}+\rho_{d}{ }^{\prime} w\right.}
$$

Equation 3

The terms representing the "averaged deviation from the average" are zero, so they are eliminated, leaving:

$$
F=\left(\overline{\rho_{d}} \bar{w} \bar{s}+\overline{\rho_{d}} \overline{w^{\prime} s^{\prime}}+\bar{w} \overline{\rho_{d}{ }^{\prime} s^{\prime}}+\bar{s} \overline{\rho_{d}{ }^{\prime} w^{\prime}}+\overline{\rho_{d}{ }^{\prime} w^{\prime} s^{\prime}}\right)
$$

Equation 4

For conventional EC calculations it is assumed that fluctuations in air density are negligible, so terms multiplied by these are eliminated:

$$
F=\left(\overline{\rho_{d}} \bar{w} \bar{s}+\overline{\rho_{d}} \overline{w^{\prime} s^{\prime}}+\bar{w} \overline{\rho_{d}{ }^{\prime} s^{\prime}}+\bar{s} \overline{\rho_{d}{ }^{\prime} w^{\prime}}+\overline{\rho_{d}{ }^{\prime} w^{\prime} s^{\prime}}\right)
$$

Equation 5

Another assumption is that the mean vertical flow is negligible (for horizontal homogenous terrain) and so the average "w" terms drop out:

$$
F=\overline{\rho_{d}} \bar{w} \bar{s}+\overline{\rho_{d}} \overline{w^{\prime} s^{\prime}}
$$

Equation 6

Leaving the final equation for the approximate "eddy flux":

$$
F \approx \overline{\rho_{d}} \overline{w^{\prime} s^{\prime}}
$$

Equation 7

For these equations to be applicable some critical assumptions must be made. A few of the key assumptions required for EC analysis include:

- Measurements at a point represent an upwind area (footprint or fetch)

- Measurements are completed in the boundary layer of interest and in constant flux layer

- Fetch and footprint are adequate representations of the area of interest

- Flux is fully turbulent

- Terrain is horizontal and uniform

- No air density fluctuations

- Flow divergences and convergences are negligible

- Instruments can detect small changes at high frequency

- Mean air flow and turbulence at the measurement point are not significantly distorted by instruments [64]. 
The assumptions above are in general necessary for any type of EC calculations. The steps involved in processing EC data are complex and detailed. Accurate EC flux results involve a complex series of preconditioning, applying corrections, and averaging. These include but are not limited to:

$\begin{array}{ll}\text { - } & \text { Unit conversion } \\ \text { - } & \text { Despiking } \\ \text { - } & \text { Apply calibrations } \\ \text { - } & \text { Rotation of axes } \\ \text { - } & \text { Time Delay corrections } \\ \text { - } & \text { De-trending } \\ \text { - } & \text { Averaging } \\ \text { - } & \text { Frequency response } \\ \text { - } & \text { Sonic corrections } \\ \text { - } & \text { Webb-Pearman-Leuning Terms } \\ \text { - } & \text { Quality control } \\ \text { - } & \text { Gap-filling } \\ \text { - } & \text { Integration }\end{array}$

The specific methods used to carry out these calculations for this research are covered in more detail in 3.2.2. Even with these assumptions, however, the EC method can produce inaccurate results due to poor design or calculation errors. The assumptions and errors typically associated with EC experiments were developed by taking measurements over mostly flat, homogeneous terrains. Hilly terrains, obstructions to flow, or heterogeneous fluxes have historically resulted in less accurate EC measurements.

EC experiments are often conducted with the same general data acquisition setup. The most basic requirements for measuring $\mathrm{EC}$ data for methane are an ultrasonic anemometer and a methane gas analyzer. The capabilities of the instruments used must meet certain criteria to achieve accurate measurements. The instruments must be "fast" meaning that they are capable of recording variables at a rate of at least $10 \mathrm{~Hz}$. Gas analyzers can be either open- or closed-path and both have their advantages depending on the situation. Open path analyzers have the advantage of being fast measuring with excellent frequency response. They often have low power requirements and have little sensitivity to window contamination and do not require frequent calibration. One major drawback of open-path analyzers, however, is data loss during precipitation or significant fog. Closed-path analyzers on the other hand rarely experience data loss due to weather, however, they experience frequency losses and usually require significantly more power due to their use of a pump to draw in the sample.

Typical EC instrumentation also often includes weather sensors and $\mathrm{H}_{2} \mathrm{O}$ analyzer. A full EC setup should also include instrumentation to measure the methane concentration profile (a slow analyzer) and solar loading detection, and possibly soil sensors. The setup of the EC instruments is also important and can impact results. The gas analyzer should be placed as closely as possible to the sonic anemometer, without causing significant interference. Burba et al. recommended positioning the measurement point of the gas analyzer (whether it be the center of the path for open-path analyzers, or the sample inlet for closed-path analyzers) at or below the measurement point of the sonic anemometer, and to minimize horizontal separation. It is suggested that the gas analyzers be placed to the side of the anemometer so that they do not disturb the wind from the dominate direction. Ultimately the selection of instruments and their placement depends on the objectives of the experiment.

The placement of the entire system is also critical to method success. EC measurements are typically made with a "tower" meaning that all the instrumentation is connected to a freestanding tower of some kind, however, the instrumentation could be attached to any elevated plane that produces minimal flow interference. The location of this tower is likely the most important aspect of EC experimental design. The tower must be able to measure the area of interest. The area that the tower is capable of measuring is often 
called the "fetch" or "footprint". This area is governed by atmospheric variables and instrument location and height. The instrumentation should be positioned so that the fetch includes the area of interest during as many wind conditions as possible, but at a minimum for prevailing winds. Therefore, EC towers should be positioned downwind of the area of interest and at a height high enough to include the area. The size of the footprint measured depends on the height of the tower, as well as the surface roughness and thermal stability [64]. The size of the footprint generally increases with increased measurement height, decreased roughness, or shifts from unstable to more stable. The footprint is a critical component of EC and several studies have been conducted to improve footprint definition and quantify fluxes from point sources [66][68].

The goal of this research, with respect to EC was to examine the possibility that the addition of such measurements could improve estimates of conventional indirect quantification methods at complex NG infrastructure sites. The intention was not to alter methodology, data collection, or calculation methods, but to use the results of this method combined with other indirect methods, such as OTM, to reduce the uncertainty associated with indirect quantification. While most NG well pads are relatively open and flat, especially in the western US, they are also sources of heterogeneous, temporal emissions. The nature of these emissions is not like those conventionally measured by EC. Therefore, the design of the DAQ system used in this research reflects a combination of the most conventional EC setup with that of OTM. More details are presented in 3.1.1.

Numerous studies have investigated methane fluxes from a variety of terrains and eco-systems. While most of these were not representative of a NG well pad, in terms of emissions or ecology, they provide order of magnitude comparisons. Common applications of EC for the purpose of quantifying methane emissions were rice paddy fields, wetlands, boreal (subarctic) lands, landfills, and other croplands. Table 14 presents a variety of methane fluxes measured via the EC method. Many of these studies were previously outlined by Tang et al [69]. The studies have shown methane fluxes from as little as 0.0013 grams of carbon from methane per square meter per day $\left(\mathrm{g} / \mathrm{m}^{2}\right.$-day) from a wetland in Canada to as high as $144 \mathrm{~g} / \mathrm{m}^{2}$-day from a beef cattle feedlot in the US [70]-[72]. The results from these studies presented in Table 14 represent a clear bifurcation between anthropogenic and biogenic sources. The top four flux sources (landfills and beef cattle feedlots) represent anthropogenic sources. The smallest of these sources was over two orders of magnitude greater than the largest biogenic source. The beef cattle feedlot was three orders of magnitudes higher than any biogenic source measured by these studies. 
Table 14. Summary of methane fluxes measured by EC from previous studies.

\begin{tabular}{|c|c|c|c|c|}
\hline Lead Author & Ecosystem & Country & Year & $\begin{array}{c}\mathrm{CH}_{4} \text { flux } \\
\text { (g/m2-day) }\end{array}$ \\
\hline Emmerton & Wetland & Canada & 2014 & 0.0013 \\
\hline Smeets & Ponderosa pine & United States & 2007 & 0.0025 \\
\hline McDermitt & Grass Wetland & United States & 2011 & 0.0036 \\
\hline Chu & Soybean cropland & United States & $2011-12$ & 0.0063 \\
\hline Wille & Arctic tundra & Russia & $2003-04$ & 0.0066 \\
\hline Detto & Degraded peatland pasture** & United States & 2011 & 0.0069 \\
\hline Peltola & Nutrient-poor oligotrophic open fen & Finland & 2013 & 0.01 \\
\hline Hargreaves & Aapa mire* & Finland & $1995 / 97 / 98 / 08$ & 0.011 \\
\hline Hartley & Aapa mire* & Finland & 2008 & 0.013 \\
\hline Knox & Rice paddy & United States & $2012-13$ & 0.014 \\
\hline Alberto & Rice fields & Philippines & $2012-13$ & 0.018 \\
\hline Tseng & Rice paddy & Taiwan & 2006 & 0.018 \\
\hline Long & Boreal fen & Canada & 2007 & 0.019 \\
\hline Tang & Trapical peat forect & Malaysia & 2013 & 0.024 \\
\hline Rhinne & Boreal fen & Finland & $2005-06$ & 0.026 \\
\hline Podgrajsek & Boreal lake & Sweden & 2016 & 0.028 \\
\hline Sturtevant & Lakes and vegetation & United States & 2013 & 0.028 \\
\hline Knox & Pasture & United States & $2012-13$ & 0.031 \\
\hline Detto & Paddy rice field & United States & 2011 & 0.042 \\
\hline Jackowicz-Korczynski & Subarctic peatland & Sweden & $2006-07$ & 0.056 \\
\hline Song & Alpine wetland & China & $2011-13$ & 0.062 \\
\hline Chamberlain & Subtropical pastures & United States & $2013-15$ & 0.064 \\
\hline Knox & Restored old wetland & United States & $2012-13$ & 0.11 \\
\hline Philipp & Subtropical grass marshland & Taiwan & 2015 & 0.11 \\
\hline Chu & Freshwater marsh & United States & $2012-13$ & 0.14 \\
\hline Knox & Restored young wetland & United States & 2011-13 & 0.15 \\
\hline Detto & Restored wetland & United States & 2011 & 0.26 \\
\hline Kowalska & Temperate wetland & Poland & 2013 & 0.32 \\
\hline $\mathrm{Xu}$ & Landfill & United States & 2014 & 25 \\
\hline Lohila & Municipal landfill & Finland & 2007 & 46 \\
\hline Riddick & Landfill & $\begin{array}{c}\text { United } \\
\text { Kingdom } \\
\end{array}$ & 2018 & 61 \\
\hline Prajapati & Beef feedlot $(58,000$ cattle $)$ & United States & 2017 & 144 \\
\hline
\end{tabular}

*Aapa mire is Finnish for cold climate wetland

** cows present

While EC, has not conventionally been used for flux measurements at NG sites. The AmeriFlux Management Project sponsored by the US DOE deemed 2019 the "Year of Methane" which was its first themed year and focused on "encouraging more methane EC measurements and data contributions" [73].

Several studies have examined the use of various "flux footprint models" with the aim of quantifying point source emissions or emissions from specific areas. These types of analyses have generally been used for 
animal agriculture studies with the goal of estimating emissions from specific animals (cattle) or areas (feedlots). However, the principle of such methods could apply to any concentrated emissions source.

Three of the most used EC flux footprint models are those developed by Hsieh et al., Kormann and Meixner, and Kljun et al. [74]-[76]. These models are all relatively new, being developed after the turn of the century. Modern computational power allowed models to become more complex. Several footprint models were also developed in the 1980's and 90's from which many of these models are based [77]-[79]. All models are generally formulated around solutions to traditional advection-diffusions equations. The equations are often solved using either a Eulerian or Lagrangian frameworks with analytic, numerical, or stochastic methodologies.

The models developed by Kormann and Meixner and Kljun et al. have been compared by several researchers in previous studies [66], [71], [80], [81]. The conclusions of these studies have not been conclusive in determining whether one model is better overall, as results from studies have varied depending on the setup, measurement goals, and micrometeorological conditions.

Hsieh et al. developed a model which was based on the Lagrangian stochastic dispersion model results with a dimensional analysis [74]. The goal of their research was to develop an analytic expression for the footprint based on observation height, surface roughness and atmospheric stability. The model developed built upon historically used Eularian models by Gash (1986), Horst and Weil (1994) and Thomson (1987). The resultant model was proposed for measurement heights between 2 and $20 \mathrm{~m}$, surface roughnesses between 0.01 and $0.1 \mathrm{~m}$ and Monin-Obukhov lengths between -0.1 and $50 \mathrm{~m}$. Curve fits based on different atmospheric stabilities (unstable, neutral, and stable) were used to relate the dimensionless distances and the proposed footprint function was based on these relationships [74].

Kormann and Meixner also developed an analytical model that was based on two-dimensional advectiondiffusion equations and utilized power law profiles of mean wind velocity and eddy diffusivity. The authors presented a detailed derivation of their model, which was primarily based on well documented methods. The authors state that their formulations are "simple and analytically treatable under all conditions of atmospheric stability." [75]. Details of the model are summarized well by Prajapati and Santos, who present the two-dimensional footprint function for a given measurement height as presented here by Equation 8 $[72]$.

$$
\phi_{K M}=\left[\frac{1}{\sqrt{2 \pi} D x^{E}} e^{\left(\frac{-y^{2}}{2\left(D x^{E}\right)^{2}}\right)}\right] C x^{-A} e^{\left(\frac{-E}{x}\right)}
$$

Equation 8

Where $A, B, C, D$, and $E$ are various functions that depend on the measurement height above the displacement height, the friction velocity, the Monin-Obukhov length, the standard deviation of the crosswind component, the wind direction, and the mean horizontal wind speed. The values $x$ and $y$ represent the upwind location of a unit point source.

Kljun et al. first presented a footprint model in 2004 and later updated their "Flux Footprint Prediction" (FFP) model in 2015. They present details of the derivations in both studies [76], [77]. The Kljun model, often referred to in the literature as FFP, provided two-dimension solutions that are implementable without extreme computational costs. The authors stated that FFP was less restrictive than other footprint models. The model was developed with simulations of the backward Lagrangian stochastic particle dispersion model (LPDM-B). The FFP model was therefore limited by the simulations the LPDM-B model could produce. The limitations of the model were summarized by two primary conditions, which are represented by Equation 9 and Equation 10.

$$
20 z_{0}<z_{m}<h_{e}
$$




$$
-15.5 \leq \frac{Z_{m}}{L}
$$

Equation 10

Where $z_{0}$ is the surface roughness, $z_{m}$ is the measurement height, $h_{e}$ is the entrainment height, which is typically defined as about $80 \%$ of the boundary layer height, and $L$ is the Monin-Obukhov length. With the presentation of their 2015 model the authors compared the FFP to models developed by Hsieh et al., Kormann and Meixner and their original 2004 model for several scenarios with varying micrometeorological conditions and receptor heights. The models of Hsieh et al. and Kormann and Meixner resulted in footprints 1.5 to 2 times larger than those of FFP for neutral and stable conditions. However, in mixed layer and free convection layer scenarios the opposite was true. This fact alone shows that models are sensitive to micrometeorological conditions. Kormann and Meixner also predicted wider footprints than FFP under most conditions. The FFP of 2015 and 2004 agreed relatively well as was expected since the models were similar [76]. The FFP model was summarized well by Prajapati and Santos as presented in Equation 11 [72].

$$
\phi_{F F P}=\overline{f^{y}}(x) \frac{1}{\sigma_{y} \sqrt{2 \pi}} e^{\left(\frac{-y^{2}}{2 \sigma_{y}^{2}}\right)}
$$

Equation 11

Where $\overline{f^{y}}$ is the crosswind integrated footprint, $\mathrm{y}$ is the crosswind distance from the $\mathrm{x}$ axis of the footprint and $\sigma_{\mathrm{y}}$ is the standard deviation of the crosswind distance.

More recent studies have taken to utilizing previously developed footprint models to estimate point source emissions. For example, Heidbach et al. analyzed the models of Schmid (1994), Hsieh et al. (2000), Kormann and Meixner (2001), and Kljun et al. (2015) [80]. To analyze the effectiveness and uncertainty of the footprint methods, the researchers utilized a fixed-point emissions source at distances of $35 \mathrm{~m}$ and 20 $\mathrm{m}$ from their EC tower. The emissions source was methane and was released in 10-minute increments at rates varying from 6 to $8.5 \mathrm{slpm}$. The experiments were conducted in a field surrounded by trees in Germany with a median roughness length of $0.03 \mathrm{~m}$. The simulated emissions were only released during the daytime. EddyPro ${ }^{\circledR}$ was used to calculate methane fluxes and an averaging period of 10-minutes was used. The researchers showed that low frequency losses at this averaging interval were negligible compared to the more commonly used 30-minute intervals. The researchers only utilized flux data when the prevailing wind direction was $\pm 40^{\circ}$ of the source to sensor (StS) direction. The researchers attempted to quantify uncertainty from both the flux measurements and the footprint models. For the uncertainty of the flux, they used the approach outlined by Richardson et al [82]. The uncertainty of the footprint models was assumed to be from three sources: incomplete model theory, violation of model assumptions, and uncertainty of input parameters. It was assumed that the uncertainty of friction velocity and Obukhov length, which are required inputs, produce large random errors. The researchers then followed the method of Finkelstein and Sims (2001) (F\&S) to determine the random error and propagated those errors to determine model uncertainty [83]. A summary of reported model uncertainties from the study and overall assessments of source location estimation are presented in Table 15. They determined that the Kljun et al. (2015) model worked the best overall, based on their assessment of RMSE of source location. The RMSE and $\mathrm{R}^{2}$ findings presented here were based on 216 observations that were not made near a forest edge. 
Table 15. Summary of footprint models and their performance as reviewed by Heidbach et al [80].

\begin{tabular}{|c|c|c|c|c|c|c|}
\hline $\begin{array}{c}\text { Footprint } \\
\text { Model Source }\end{array}$ & $\begin{array}{c}\text { Uncertainty } \\
\text { at Footprint } \\
\text { Maximum } \\
\text { (\%) }\end{array}$ & $\begin{array}{c}\text { Uncertainty } \\
\text { Minimum } \\
\mathbf{( \% )}\end{array}$ & $\begin{array}{c}\text { Uncertainty } \\
\text { Minimum } \\
\text { Distance (\%) } \\
\text { of Max) }\end{array}$ & $\begin{array}{c}\text { \% Cases of } \\
\text { Underestimation }\end{array}$ & $\begin{array}{c}\text { RMSE } \\
\left(\mathbf{m}^{-2}\right)\end{array}$ & $\mathbf{R}^{\mathbf{2}}$ \\
\hline Schmid (1994) & 13 & 9 & 140 & $69 \%$ & $3.8 \times 10^{-4}$ & 0.3 \\
\hline $\begin{array}{c}\text { Kormann and } \\
\text { Meixmer } \\
(2001)\end{array}$ & 27 & 5 & 190 & $71 \%$ & $4.7 \times 10^{-4}$ & 0.2 \\
\hline $\begin{array}{c}\text { Hsieh et al. } \\
(2000)^{*}\end{array}$ & 48 & 3 & 250 & $62 \%$ & $3.9 \times 10^{-4}$ & 0.26 \\
\hline $\begin{array}{c}\text { Kljun et al } \\
(2015)\end{array}$ & 40 & 0.30 & 360 & $50 \%$ & $3.3 \times 10^{-4}$ & 0.16 \\
\hline
\end{tabular}

*biased, would be much worse at night

Parajapati and Santos evaluated the KM and FFP models to estimate methane emissions rates of beef cattle in a feedlot [72]. They utilized a tower with a height of $5 \mathrm{~m}$ and processed flux data in 30-miunte intervals using EddyPro ${ }^{\circledR}$. The surface roughness during data collection generally ranged from 1 to $10 \mathrm{~cm}$. Fetch comparisons were performed using the two models by comparing footprints with the same normalized integral footprint contribution (P). For equal values of P more data were retained from the FFP model compared to the KM model. This indicated that the KM model tended to overestimate source area compared to FFP and the researchers found that KM produced footprint areas 5-6 times larger on average. They used the source weight distribution of Schmid (1994) to determine the outer limit of the source area, which was defined as the distance contributing $1 \%$ of the maximum source area contribution. These values were 0.8 for FFP and 0.7 for KM. Differences between the two models varied significantly because of atmospheric stability conditions. The downwind distance contributing $80 \%$ of the flux under unstable conditions was 1.5 times greater when using KM compared to FFP. For neutral and stable conditions, the differences increase to 2.3-3.0 times on average. Most of the data were collected under neutral and stable conditions which could have resulted in larger differences between the models. The average crosswind source area estimated by KM was also 2 times larger than that of FFP. The differences agreed with conclusions from Kljun et al. (2015) [76].

The authors made two important notes about the need for further evaluation of footprint models:

- "field studies are needed to investigate the accuracy of those footprint models under different atmospheric conditions"

- "additional tracer release studies are necessary to improve the confidence of EC measurements and validate footprint model estimates" [72].

Wilson et al. (2015) compared the KM model to a random displacement model and a Lagrangian stochastic model and found that the KM model improved with tuning of the Schmidt number (Sc) from 1.0 to 0.64, but still found that KM misrepresented the flux footprint based on comparisons to LSM simulations [81]. This conclusion was supported by other studies [76], [84].

Coates et al. (2017) used controlled releases to evaluate a Lagrangian stochastic model (LSM) for its potential to estimate point source emissions from cattle [68]. The site evaluated was a $60 \mathrm{~m}$ by $60 \mathrm{~m}$ plot of land with a grass canopy height of $0.4 \mathrm{~m}$. The researchers utilized eight different point sources which distributed a total of $5 \mathrm{slpm}$ of methane at heights of $0.8 \mathrm{~m}$. Each point source released approximately 600 grams of methane per day $\left(\mathrm{gCH}_{4} / \mathrm{d}\right)$. Two EC systems were used for measurement, each consisting of a LICOR ${ }^{\circledR}$ LI-7700 and LI-7500 and a Gill® Windmaster. The systems were placed at distances of $5 \mathrm{~m}$ and 50 $\mathrm{m}$ downwind of the area. Both systems were mounted at a height of $3.6 \mathrm{~m}$. The data were processed in EddyPro ${ }^{\circledR}$ using 15-minute periods. The researchers applied filters to remove data periods which were 
flagged by Mauder and Foken (2004) [85], had an unfavorable wind direction, produced a $\mathrm{u}^{*}$ value less than 0.15 , or had a Monin-Obukhov length less than $10 \mathrm{~m}$. These filters excluded $31 \%$ of data from the near tower and $43 \%$ of data from the far tower. Most of the eliminated periods occurred during nighttime. They evaluated both 15- and 30-minute periods and found very little difference in flux estimates. The authors noted the advantage of LSMs over analytic footprint models since there was no systemic underestimation from the far tower, which produced more accurate results on average than the near tower. The authors concluded that with the combination of EC measurements from two towers, an LSM, and source position information that source emissions can be determined with an accuracy of $10 \%$. However, this method required substantially greater computational power than simpler analytic models [68].

Dumortier et al. (2019) used a single artificial methane source to evaluate the KM and FFP functions [67]. The researchers utilized an artificial methane source of $1.5 \mathrm{slpm}$ (about $1544 \mathrm{gCH}_{4} / \mathrm{d}$ ) which was released at distances of 23,80 , and $180 \mathrm{~m}$ from the EC tower. Methane was only released when the winds were $\pm 45^{\circ}$ of the StS direction and the $\mathrm{u}^{*}$ was greater than $0.13 \mathrm{~m} / \mathrm{s}$ in the previous averaging interval. The researchers utilized EddyPro ${ }^{\circledR}$ for flux calculations and tested averaging intervals of 5, 15, and 30 minutes, with and without stationarity filters. Amplitude resolution, skewness, and kurtosis tests were disabled in EddyPro® because these filters eliminated most periods with an artificial source which was believed to be due to the emissions peaks. The researchers determined emissions rates by combining flux measurements with source positions and the footprint functions analyzed by utilizing Equation 12.

$$
F_{x}=F_{i j, \text { source }} \phi_{i j, \text { source }} \Delta x_{i j} \Delta y_{i j}
$$

Equation 12

In Equation 12, $F_{i j, \text { souce }}$ is the flux density of the cell containing the source ( $\left.\mathrm{nmol} / \mathrm{m}^{2} \mathrm{~s}\right), \phi_{\mathrm{ij}}$ is the value of the footprint function in the cell $\left(\mathrm{m}^{-2}\right)$, and $\Delta x_{i j}$ and $\Delta y_{i j}$ are the $\mathrm{x}$ and $\mathrm{y}$ size of the cell containing the source (m). The authors utilized both KM and FFP to determine $\phi$. They assumed a boundary layer height of 1500 $\mathrm{m}$ and $300 \mathrm{~m}$ for day and night, respectively. The source emission and uncertainty were estimated with the slope of a linear regression between $F_{\text {source }}$ and $\phi_{\text {source }}$.

The researchers final test matrix consisted of 2 footprint models, 3 averaging periods, 2 averaging methods, and 2 modalities of the stationarity test. Each of these tests were evaluated based on an accuracy score consisting of estimated accuracy (measured as absolute error in the estimate), reproducibility (measured as the variance of measurements), and quality of relationship (measured as $\mathrm{R}^{2}$ of linear regression fit). Quality checks were analyzed by the authors to ensure that their methodology performed across a range of situations. To do this they subdivided data into various equal subsets with increasing variables of interest. The variables analyzed included friction velocity, atmospheric stability, wind direction, and variation in wind direction within the averaging period. They found no impact of atmospheric stability or wind direction variance within the period. Their findings did suggest that results may be better when wind direction is within $\pm 40^{\circ}$ of the StS direction. Periods with friction velocity greater than $0.4 \mathrm{~m} / \mathrm{s}$ tended to overestimate emissions, however, eliminating these periods did not result in better average scores.

The researchers concluded that the KM model with 15-minute averaging periods, using a running mean and no stationarity produced the best results. This methodology resulted in estimated emissions between 90$113 \%$ of actual rates, suggesting that this was a legitimate technique for elevated point-source emission quantification. They observed significant differences based on the footprint model used. The selection of KM over FFP was a different conclusion than Heidbach et al. [80] observed using an artificial source. These differences suggest that topography, micrometeorological conditions, release and measurement height and difference in measurement distance can significantly impact the effectiveness of various footprint models [67].

Stoy et al. used footprint models and the method of Dumortier et al. to attribute and estimate methane emissions to individual bison in a herd [66]. The researchers utilized a $3 \mathrm{~m}$ tower containing a LICOR LI7700 in the center of a $20 \mathrm{~m}$ by $20 \mathrm{~m}$ area of a grazing pasture. Only daytime observations were used in the analysis. The researchers utilized EddyPro ${ }^{\circledR}$ to calculate methane fluxes with settings recommended by 
Dumortier et al [67]. The Mauder and Foken (2004) quality control filter was used to discard data with a flag greater than 1. Thirty-minute averaging periods were utilized and the flux footprint was calculated using the approach of Hsieh et al. (2000) with two-dimensional extension from Detto and Katul (2006) [86]. The positions of the bison during the 30-minute window were determined based on aggregated 5minute incremental photographs which led to a spatial uncertainty of the emissions sources. The results were also subject to methane flux measurement uncertainties, defined by Deventer et al. (2019) as 6-41\% for 30-minute increments [87]. After applying quality control filters, 173 30-minute periods were analyzed. The researchers quantified spatial uncertainty by shifting potential locations within the grid for each period and conservatively estimated a spatial uncertainty of $21 \%$ and long-term methane flux uncertainty of $17 \%$. The researchers also found that methane flux was significantly and positively correlated with $\mathrm{u}^{*}$ values greater than $0.2 \mathrm{~m} / \mathrm{s}$ but not related to values less than $0.2 \mathrm{~m} / \mathrm{s}$.

Two footprint models have been studied by researchers extensively in recent years. The models developed by Kormann and Meixner (2001) and by Kljun et al. (2015) have resulted in varying results across studies, suggesting that more research is needed into when each model is at its optimal effectiveness to define the flux footprint. Methods to quantify point source emissions have been developed using EC flux data and footprint models (Dumortier et al. 2019) [67]. The usefulness of such methods is only as relevant as their uncertainties or abilities to be combined with other measurements to obtain valid results. EC measurements and calculations have many sources of uncertainty as errors can propagate in several ways. While some of these errors can be eliminated with good practice, others will always be present. If EC is to be used for more unique applications such as NG infrastructure emissions quantification, such errors and methods must be understood, and steps taken to reduce them.

\subsubsection{Aerial Flux Methods}

A widely used approach to measuring methane flux on a regional scale has been with flyover aerial measurements. These methods often utilize drones, planes, or copters to traverse areas with prominent methane fluxes. The coverage area of these techniques is governed by the extent and height of the flight and wind conditions. These methods have historically been used for making basin wide estimates of methane emissions from the NG sector. Oftentimes areas densely populated with components of the NG infrastructure, such as production sites, compressor stations, and pipeline are measured with these techniques to give "top-down" emissions estimates. Several studies have utilized this method to make estimates of regions or specific elements of the NG infrastructure.

Karion et al. published regional estimates of the Barnett shale region in 2013. The research utilized a Picarro G2401-m and focused on an area with production, processing, and distribution infrastructure. They estimated emissions to be $60 \pm 11 \times 10^{3} \mathrm{~kg} / \mathrm{hr}$ of methane [88]. Smith et al. also performed aerial flux estimates of the Barnett shale region and found agreeable results using a Picarro G2401-m. The researchers estimated the total regional methane flux to be $74 \pm 18 \times 10^{3} \mathrm{~kg} / \mathrm{hr}$ [89]. Smith et al. later performed flux estimates of the San Juan shale gas basin and estimated total basin wide emissions to be $0.54 \mathrm{Tg} / \mathrm{yr}$ [90]. Lavoie et al. performed estimations of eight specific sites within the Barnett region using five individual flights, which were also outfitted with a Picarro G2401-m for methane measurement. They estimated emissions from these eight sites, which included NG processing plants, compressor stations, and landfills to total $7000 \mathrm{~kg} / \mathrm{hr}$ [91]. Peishl et al. estimated fluxes from three major US shale plays. Their DAQ method utilized a Picarro 1301-m to measure methane concentration. The methane flux estimates from the Haynesville, Fayetteville, and Marcellus shale plays were $8.0 \times 10^{7}, 3.9 \times 10^{7}$, and $1.5 \times 10^{7} \mathrm{~g} / \mathrm{hr}$, respectively [92]. Schwietzke et al. also performed flyover measurements in the Fayetteville shale region over two days. They attempted to quantify the emissions by dividing the region into "east" and "west" segments and measured concentrations with a Picarro 2301-f. The average total regional methane flux estimate from the study was 27.3 tons/hr [93]. Petron et al. also performed measurements over two days using a Picarro in the DJ basin. They estimated a regional methane flux of 19.3 tons/hr [94]. In 2015 Karion et al. published regional estimates of methane emission in Unitah County, Utah. This time the researchers utilized highresolution doppler LiDAR and estimated a total methane flux of $54.6 \times 10^{3} \mathrm{~kg} / \mathrm{hr}$ [95]. Caulton et al. 
performed regional flux estimates in the Marcellus region and estimated methane emissions to be between 2 and $14 \mathrm{~g} / \mathrm{s}$ per $\mathrm{km}^{2}$ over an area of $2,800 \mathrm{~km}^{2}$ [96].

A more in-depth modeling analysis of the Marcellus shale region of northwestern PA was performed by Barkley et al. In this campaign, researchers performed ten flights over a three-week period using a Picarro G2401-m. The estimated emissions by sectors of the NG infrastructure using a Monte-Carlo analysis. The total methane emission estimate of the region was $2.76 \mathrm{Tg} / \mathrm{yr}$. The researchers estimated that $\sim 0.73 \mathrm{Tg} / \mathrm{yr}$ of these emissions were from unconventional and conventional wells [97].

Englander et al. also estimated emissions specifically from well pads in the Bakken region. They combined observations from a helicopter team outfitted with FLIR ${ }^{\circledR}$ cameras with flyover measurements utilizing Picarro concentration measurement between 2014 and 2015. In total, they estimated regional methane emissions to be greater than $25,000 \mathrm{~kg} / \mathrm{hr}$, attributing more than $20,000 \mathrm{~kg} / \mathrm{hr}$ to well pads. Their helicopter crew reported that the percentage of wells with observable leaks was $14 \%$ and $11 \%$ in the years 2014 and 2015 , respectively. The study also highlighted the skewed distribution of emissions. They approximated that the highest $7.7 \%$ of well pads contributed $56 \%$ of emissions [98].

A summary of these studies is presented in Table 16. Many more studies have been performed in a similar manner over the course of the 2010s. One of fallbacks of these methods, however, is the problem of source attribution. Estimates are often made using atmospheric methane concentrations. If there are other potential sources of methane in the area, it can be difficult to attribute emissions to the NG infrastructure. As was observed by the EC studies, wetlands, landfills, and feed lots may be significant sources of methane emissions. Interference of such sources hinders an aerial measurements' ability to attribute methane to NG sources. This leads to higher uncertainty in measurements from the NG infrastructure and could lead to erroneous emissions inventories.

Table 16. Summary of aerial regional methane flux estimates from various studies.

\begin{tabular}{|c|c|c|c|}
\hline Study & $\begin{array}{c}\text { Year } \\
\text { Published }\end{array}$ & Region & $\begin{array}{c}\text { CH4 Flux } \\
\text { Estimate } \\
\text { (kg/hr) }\end{array}$ \\
\hline Karion et al. & 2013 & Barnett & $6.0 \times 10^{4}$ \\
\hline Caulton et al. & 2014 & Marcellus & $2.0 \times 10^{4}$ \\
\cline { 3 - 4 } & & DJ & $1.4 \times 10^{5}$ \\
\hline Petron et al. & 2014 & $1.8 \times 10^{4}$ \\
\hline Karion et al. & 2015 & Uintah, UT & $5.5 \times 10^{4}$ \\
\hline Lavoie et al. & 2015 & Barnett & $7.0 \times 10^{3}$ \\
\hline & & Haynesville & $8.0 \times 10^{4}$ \\
\cline { 3 - 4 } Peishl et al. & 2015 & Fayetteville & $3.9 \times 10^{4}$ \\
\cline { 3 - 4 } & & Marcellus & $1.5 \times 10^{4}$ \\
\hline Smith et al. & 2015 & Barnett & $7.4 \times 10^{4}$ \\
\hline Barkley et al. & 2017 & Marcellus & $3.2 \times 10^{5}$ \\
\hline Schwietzke et al. & 2017 & Fayetteville & $2.5 \times 10^{4}$ \\
\hline Smith et al. & 2017 & San Juan & $6.2 \times 10^{4}$ \\
\hline Englander et al. & 2018 & Bakken & $2.5 \times 10^{4}$ \\
\hline
\end{tabular}




\subsubsection{Latest Advances in Methane Quantification and Leak Detection}

With the emergence of an increased focus on methane emissions from the US NG infrastructure, several more novel techniques have emerged in recent years. Two funding campaigns have specifically focused on novel methods to identify and quantify leaks with the goal of methane emissions reductions. The Stanford/EDF Mobile Monitoring Challenge and the DOE ARPA-E MONITOR program have supported a number of methods that have been tested in recent years [4], [99].

Ravikumar et al. reviewed the results of the Stanford study, which funded ten different mobile platforms (vehicle, drone, and plane). The technologies were evaluated on three different levels based on proximity and accuracy of leak identification. They were also evaluated based on ability to quantify the leaks, although this was not the goal of the research. The quantification results were based on the percentage of estimations that were within 0.5-2 times and 0.1-10 times actual emissions rate. Quantification accuracy was calculated by dividing the measured emission rate by the actual. A summary of the quantification results is presented in Table 17 [100].

Table 17. Methane measurement technologies from Stanford/EDF Mobile Monitoring Challenge [100].

\begin{tabular}{|c|c|c|c|c|}
\hline \multirow{2}{*}{ Company } & \multirow{2}{*}{$\begin{array}{l}\text { Platform } \\
\text { Type }\end{array}$} & \multirow{2}{*}{ Survey Method } & \multicolumn{2}{|c|}{$\begin{array}{l}\text { Quantification } \\
\text { Accuracy }(\%)\end{array}$} \\
\hline & & & $\begin{array}{l}0.5-2 \\
\text { times }\end{array}$ & $\begin{array}{c}0.1-10 \\
\text { times }\end{array}$ \\
\hline ABB/ULC Robotics & Drone & Modified raster scan (wind responsive) & 30 & 78 \\
\hline Advisian & Helicopter & $\begin{array}{l}\text { Upwind/Downwind transects with sample } \\
\text { tube }\end{array}$ & 25 & 79 \\
\hline Aeris Technologies & Vehicle & Fence-line around equipment groups, facility & 38 & 79 \\
\hline Baker Hughes (GE) & Drone & Concentric circles around equipment & 24 & 54 \\
\hline Ball Aerospace & Plane & Fly-overs (multiple passes) & 53 & 83 \\
\hline Heath Consultants Inc. & Vehicle & Fence-line around equipment groups, facility & 48 & 95 \\
\hline Picarro & $\begin{array}{l}\text { Drone and } \\
\text { Vehicle }\end{array}$ & Upwind/Downwind transects & 45 & 92 \\
\hline Seek Ops Inc. & Drone & Raster scan, with flux plane mapping & 36 & 100 \\
\hline U Calgary & Vehicle & Fence-line and highway-based screening & 18 & 74 \\
\hline
\end{tabular}

Bell et al. reviewed 12 technologies that were tested at the METEC facility which was funded by the ARPAE MONITOR program. Half of the technologies were developed under ARPA-E MONITOR and half were non-MONITOR funded. Two types of test protocols were used to evaluate different solutions. The solutions evaluated were divided into "survey" solutions which were designed to be deployed within a site during a scheduled visit or "continuous monitoring" solutions which were designed to be deployed for an extended period. The solutions ranged in scale from a single site to multiple sites depending on the method of detection. The evaluation tests ranged in complexity from single continuous sources to multiple continuous sources to multiple intermittent and/or continuous sources. Of the 12 technologies evaluated, seven included a quantification mode. Quantification mass flow rates were reported for 143 simulated emissions tests. An error was reported as the difference between estimated and actual leak rates. Forty-three percent of the quantification measurements underestimated emissions and $57 \%$ overestimated emissions. The mean error of all measurements was $1.3 \mathrm{scfh}$ but the mean error from continuous monitoring solutions was $8.8 \mathrm{scfh}$. Continuous monitoring methods overestimated $75 \%$ of the time. The results of the study indicated that quantification methods were likely to produce a high uncertainty and a positive bias of $17-170 \%$. The researchers suggested that "more extensive testing focused on the accuracy and uncertainty of quantification 
methods is needed if these solutions are to inform operators or regulators of overall emissions from oil and gas operations" [101].

Golston et al. reported on the ability of an unmanned aerial vehicle to detect and quantify leaks at the METEC facility. Their system utilized an infrared backscatter tunable diode laser absorption spectroscopy, which measure methane concentrations on a path integrated scale. Three controlled release experiments were performed. The researchers noted that quantification was more difficult than leak detection. The greatest sources of uncertainty included sensor position uncertainty, concentration noise, and wind direction. The authors concluded that the algorithms could form the basis of a continuous monitoring system which would capture a large fraction of emissions [102].

Travis et al. developed an Artificial Neural Network (ANN) which was used with data collected by Aeries Technologies, Inc. as part of the MONITOR program. Their system consisted of multiple stationary points of methane measurements using a new micro-methane analyzer and a miniature 3-D sonic anemometer. The ANN inputs were patterns of methane source locations and leak rates, along with wind speeds and time. The output of the ANN was a time series of methane concentrations at the measurement locations. Input data was generated with a turbulent transport model combined with collected field data. The researchers then utilized the results to solve an inverse problem which utilized an amoeba algorithm. The site was masked to ensure that estimated leaks were only in the area of equipment. This step was critical as it reduced the possibility of significant source attribution error. The ANN and sensor network was tested at the METEC facility and could locate leaks to within $\pm 10 \%$ of the test domain. The ANN generally overestimated rates by an average factor of 0.77 (full range from 1 to 5 times the leak rate). The ANN did not perform well when there were multiple leaks present, leaks were intermittent, or when the leak rate was less than five scfh [103].

Fox et al. performed an extensive analysis of methane leak detection and quantification technologies in 2018 [104]. Their review encompassed publications focused on leak detection, mitigation, and quantification. The technologies were subdivided into six primary categories: handheld instruments, fixed sensors, mobile ground laboratories, unmanned aerial vehicles (UAVs), aircraft, and satellites. The reviews were based on four different possible goals:

M1. to develop and refine emissions inventories

M2. to estimate top-down emissions of regions

M3. close range LDAR with handheld devices

M4. rapid screening of anomalous emissions events.

Clearly their work covered a broad range of technologies but one general theme across all of them according to the reviewers was that "uncertainties were poorly constrained". The researchers sorted the technologies by spatial and temporal scale. The categorization is presented in Figure 9. It is noteworthy for this research that stationary sensors cover the lower end of spatial scales (component to facility level) but the higher end of temporal scale (month to multiple years). 


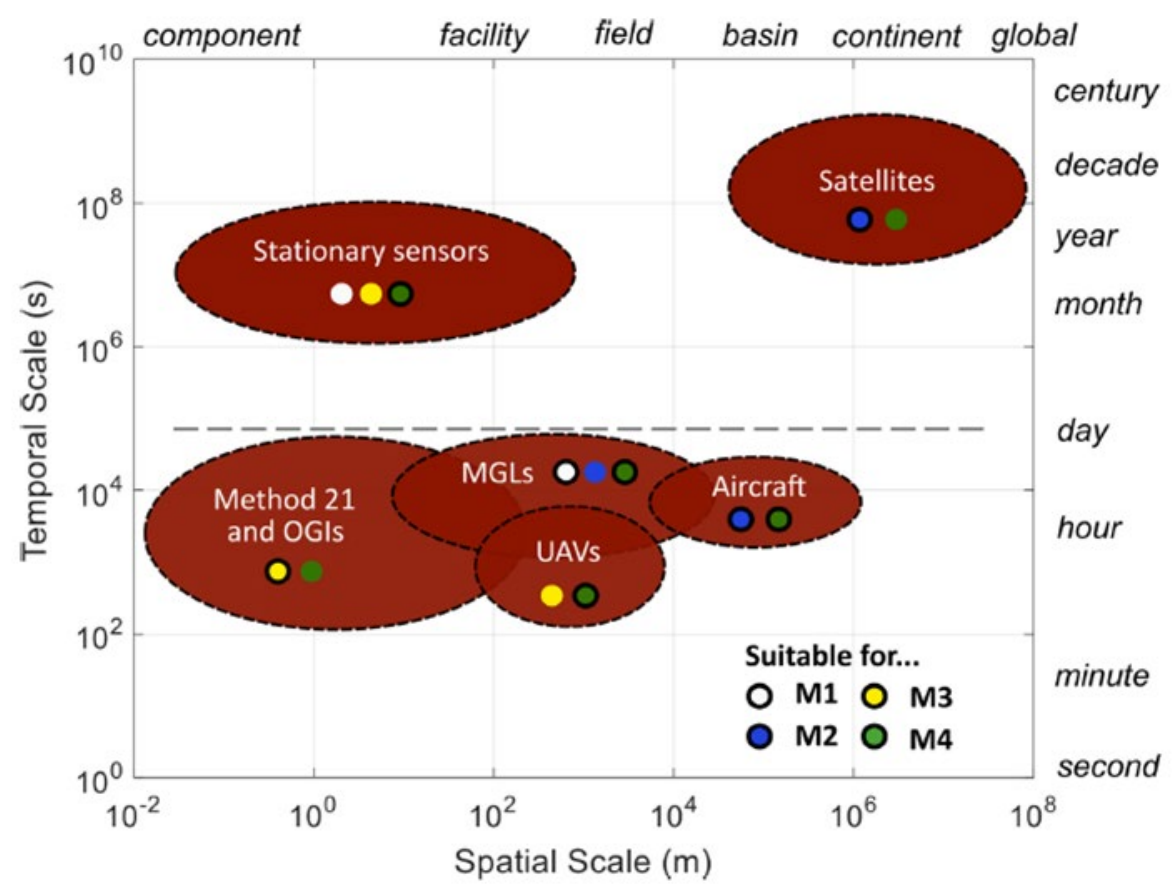

Figure 9. Temporal and spatial scales of methane detection and quantification technologies [104].

Based on their comprehensive review, there are tradeoffs to each of the technologies reviewed. Satellites can screen for large leaks across entire continents but provide little to no facility level information, while handheld devices and OGI cameras offer precise leak location information when used correctly but would require multiple years to monitor all components at NG production sites and have a small temporal scale. The detection limits, rapidity of use, accuracy and spatial and temporal scales vary widely across these technologies. A detailed summary of the review of all technologies published by the authors is presented in Figure 10. The authors noted that reported uncertainties should be interpreted with caution for several reasons because "not enough work has been done to evaluate the performance of screening technologies". The authors also concluded with two statements relevant to the research of this work.

1. "There is a pressing need for independent research to critically evaluate the strengths and limitations of screening methods, with attention to the development of detection probability curves that account for realistic environmental and operation conditions."

2. "Multiplatform comparisons of the same plumes would lead to an improved understanding of temporal variability and could greatly improve localization and quantification capabilities" [104]. 


\begin{tabular}{|c|c|c|c|c|c|c|c|c|c|}
\hline & Method 21 & OGIs & Fixed sensors & $\begin{array}{l}\text { MGLs- } \\
\text { stationary }\end{array}$ & MGLs-tracer ${ }^{2}$ & $\begin{array}{l}\text { MGLs- } \\
\text { mobile }\end{array}$ & UAVs & $\begin{array}{l}\text { Aircraft-facility- } \\
\text { scale }\end{array}$ & Satellites-facility-scale \\
\hline Limit of detection $\left(\mathrm{g} \mathrm{h}^{-1}\right)^{\mathrm{b}}$ & $<1^{c}$ & $20^{\mathrm{d}}$ & $96^{e}$ & 9-36 & $700-1.2 \times 10^{4}$ & 6-2124 & $39.6^{f}$ & $2000-4.6 \times 10^{4}$ & $2.5 \times 10^{5}-68 \times 10^{68}$ \\
\hline Flux estimation uncertainty $(\%)^{\mathrm{h}}$ & - & $3-15^{i}$ & 31 & $25-60$ & $20-50$ & $50-350$ & $25-55$ & $1-24$ & j \\
\hline Horizontal distance from source (m) & 0 & $3-6$ & $0-1000$ & $10-200$ & $500-3000$ & $5-500$ & $0-194$ & $0-1000$ & - \\
\hline Vertical distance from source (m) & - & - & - & - & - & - & $6.5-122$ & $60-1000$ & $5.12 \times 10^{5}-8.24 \times 10^{5}$ \\
\hline Time per well pad (minutes) ${ }^{k}$ & $240-960^{1}$ & $120-480$ & - & $15-20$ & $60-300$ & $0.5-5$ & $5-15$ & $5-30$ & $<0.01$ \\
\hline Sensor in plume? & Yes & No & Yes & Yes & Yes & Yes & Yes & No & No \\
\hline Component-level confirmation? & Yes & Yes & No & No & No & No & No & No & No \\
\hline Regulatory acceptance for LDAR? ${ }^{m}$ & Yes & Yes & No & No & No & No & No & No & No \\
\hline Readinesslevel for LDAR & High & High & Moderate & - & - & Moderate & Moderate & Moderate & Low \\
\hline $\begin{array}{l}\text { Number of operational commercial systems for use in } \\
\operatorname{LDAR}^{\mathrm{n}}\end{array}$ & $20+$ & $5+$ & $20+$ & - & - & $10+$ & $20+$ & $10+$ & 1 \\
\hline References and important publications & $1-3$ & $3-7$ & $8-9$ & $10-12$ & $13-15$ & $16-20$ & $21-23$ & $24-28$ & 29-30 \\
\hline
\end{tabular}

${ }^{2}$ Deemed unsuitable for screening, see section 4.3.

b These are examples of specific studies under specific conditions; more research is needed.

" Instrument-dependent.

d $90 \%$ probability ofdetection at $3 \mathrm{~m}$.

-At $1 \mathrm{~km}$.

${ }^{\mathrm{f}}$ For measurements taken within $10 \mathrm{~m}$

${ }^{8}$ Theoretical detection limits.

${ }^{\text {h }}$ This row is largely adapted from 17.

'OGI quantification is complex and uncertainties are likely much higher.

i There are no published uncertainty estimates for facility-scale emissions.

${ }^{k}$ Measurement time.

Assuming Method 21 is $50 \%$ as efficient as OGI.

${ }^{m}$ In Canada/US.

" Examples from a quick web search; more may exist or be in development. Companies are not listed to avoid endorsement. (1) Ellis and Lackaye 1989. (2) Yen and Horng 2009. (3) Ravikumar et al 2018. (4) ICF International 2014. (5) Gäfalk et al 2016. (6) Ravikumar and Brandt 2017. (7) Ravikumar et al 2017. (8) Coburn et al 2017. (9) Patel 2017. (12) Brantley et al 2014. (11) Lan et al 2015. (12) Robertson et al 2017. (13) Mitchell et al 2015. (14) Roscioliet al 2015. (15) Yacovitch et al 2017. (16) Yacovitch et al 2015. (17) Caulton et al 2018. (18) Atherton et al 2017. (19) von Fischer et al 2017. (20) Weller et al 2018. (21) Nathan et al 2015. (22) Barchyn et al 2017. (23) Golston et al 2018. (24) Frankenberg etal 2016. (25) Conleyet al 2017. (26) Smith et al 2017. (27) Englander et al 2018. (28) Schwie zke et al 2018. (29)Frankenberg et al 2005. (30) Jacob et al 2016.

Figure 10. Summary of methane detection and quantification technologies reviewed by Fox et al [104]. 


\subsection{Summary}

With the increase in NG production, there has been an increased interest in methane emissions from researchers, regulators, and industry. The most accurate method of emissions quantification has historically been direct quantification. However, the expanding infrastructure and need for more measurements to understand the environmental effect has rendered such techniques too costly to perform consistently. As a result, recent efforts to quantify emissions indirectly have focused on mobile and aerial flux measurements. These types of measurements can cover more sites, more quickly. However, these techniques come with increased uncertainties. These uncertainties have created significant discrepancies between bottom-up and top-down emissions estimates. Two established methods of emissions flux estimates that are "near-field" but not direct and that could help to alleviate some uncertainties are EC and OTM. Both require a singlepoint measurement of methane concentration, which when combined with wind and other micrometeorological variables, can be used to estimate an emissions flux. OTM has been the most widely used mobile flux technique for measuring methane emissions from targeted sites. However, literature has suggested that this method has a $2 \sigma$ uncertainty of $\pm 70 \%$. Researchers focusing on production sites have also presented significant evidence that emissions are stochastic, temporal, and often have fat-tailed distributions. These distributions are significant barriers to accurately estimating regional or national emissions from the NG infrastructure. The EC technique utilizes a similar DAQ system to that of OTM, but rather than being vehicle mounted it is traditionally mounted on a stationary tower. The EC flux measurement method is generally used to estimate biogenic fluxes from homogenous area sources. Some recent research, however, has presented evidence that the technique can be used to estimate emissions from anthropogenic point sources. Utilization of footprint modeling techniques has made this possible. However, due to the stochastic nature of turbulence, there will always be reasonably large uncertainties associate with EC flux measurements $( \pm 30 \%)$. When attributing emissions to a specific source, the uncertainties in modeling techniques amplify these uncertainties. Several studies have suggested that there is a need to improve the understanding of indirect methane quantification methods. As a response, government funding in these areas has increased in recent years. From these funding efforts a variety of unique solutions have been suggested, however, few of them have been deployed with economic or scientific feasibility.

\section{Methodology}

Several methods of methane emissions measurements have been presented thus far. This research focused on two distinct methods that can be utilized with similar sensor configurations and a single point of measurement. OTM requires a methane CMI and 3-D sonic anemometer to collect data from a position downwind of the source. The primary goal of OTM is to measure a horizontal flux by assuming a Gaussian emissions profile. EC systems require the same two primary instruments (a methane CMI and a 3-D anemometer) in addition to $\mathrm{CO}_{2}$ and $\mathrm{H}_{2} \mathrm{O}$ concentration measurements. The EC data acquisition system should be positioned downwind of the desired measurement area and the primary goal of the method is to measure vertical flux. These two techniques can therefore be utilized in tandem to lend greater confidence to measurements of emissions from a specific area such as one with a potential methane emissions point source.

\subsection{Data Collection}

A major goal of the NSF project was to "develop and validate a high-frequency system to collect methane plume and EC data necessary to evaluate indirect quantification methods". The DAQ system had to be capable of recording the variables required for both OTM and EC. It was also desirable to have a system that could be deployed on or near a site for a moderate time. Typically, OTM measurements are made in the span of hours, while EC measurements are typically made over several months or years. The goal was to design of system with moderate mobility so that it could be moved relatively frequently (days to weeks). The system developed to accomplish these goals was the Mobile Eddy Covariance Tower (MECT). This system was deployed during two data collection campaigns over the course of the research. The first campaign was a 3-month deployment at the JW Ruby Research Farm in Reedsville, WV [105]. The "farm" 
acted as an open space for controlled methane releases. The second campaign involved deployment of the MECT at an active production site over the course of one year. The Marcellus Shale Energy and Environment Laboratory (MSEEL) in Westover, WV was selected as the production site for deployment [106]. To verify indirect quantification techniques used at the MSEEL site, reliable direct quantification measurements were made for comparison. The direct quantification methane "audits" were performed using a proven measurement system known as the Full Flow Sampling (FFS) system [107]. Direct quantification measurements were performed periodically for comparison with indirect estimates from the MECT. More details on the data acquisition systems and data collection sites are presented in the follow sections.

\subsubsection{Mobile Eddy Covariance Tower (MECT)}

The primary method of DAQ was an in-house developed MECT. The design of this DAQ system was specific to this research, as it was intended to be semi-mobile for easy movement and deployment. The tower was mounted on a towable trailer so that it could easily be moved within and among production sites. The MECT was designed for operation without the need for an external power source. The DAQ equipment mounted on the tower was powered by a rechargeable battery bank which was maintained by two solar panels, also mounted on the trailer. This system could be transported and deployed at different sites as a single system with relative ease compared to conventional EC stationary systems. The DAQ system included a LICOR LI-7700 $\mathrm{CH}_{4}$ analyzer, LI-7500DS $\mathrm{CO}_{2} / \mathrm{H}_{2} \mathrm{O}$ analyzer, LI-200R pyranometer, a Gill ${ }^{\circledR}$ WindMaster 3-axis sonic anemometer, and an Omega iBTHx barometric pressure, temperature, and humidity sensor. The MECT as deployed in the field is presented in Figure 11. The specifications of all MECT instruments are presented in Table 19. All data from the DAQ system were recorded at a rate of 10 $\mathrm{Hz}$, except for weather variables from the Omega iBTHx which were recorded at $0.25 \mathrm{~Hz}$ resolution.

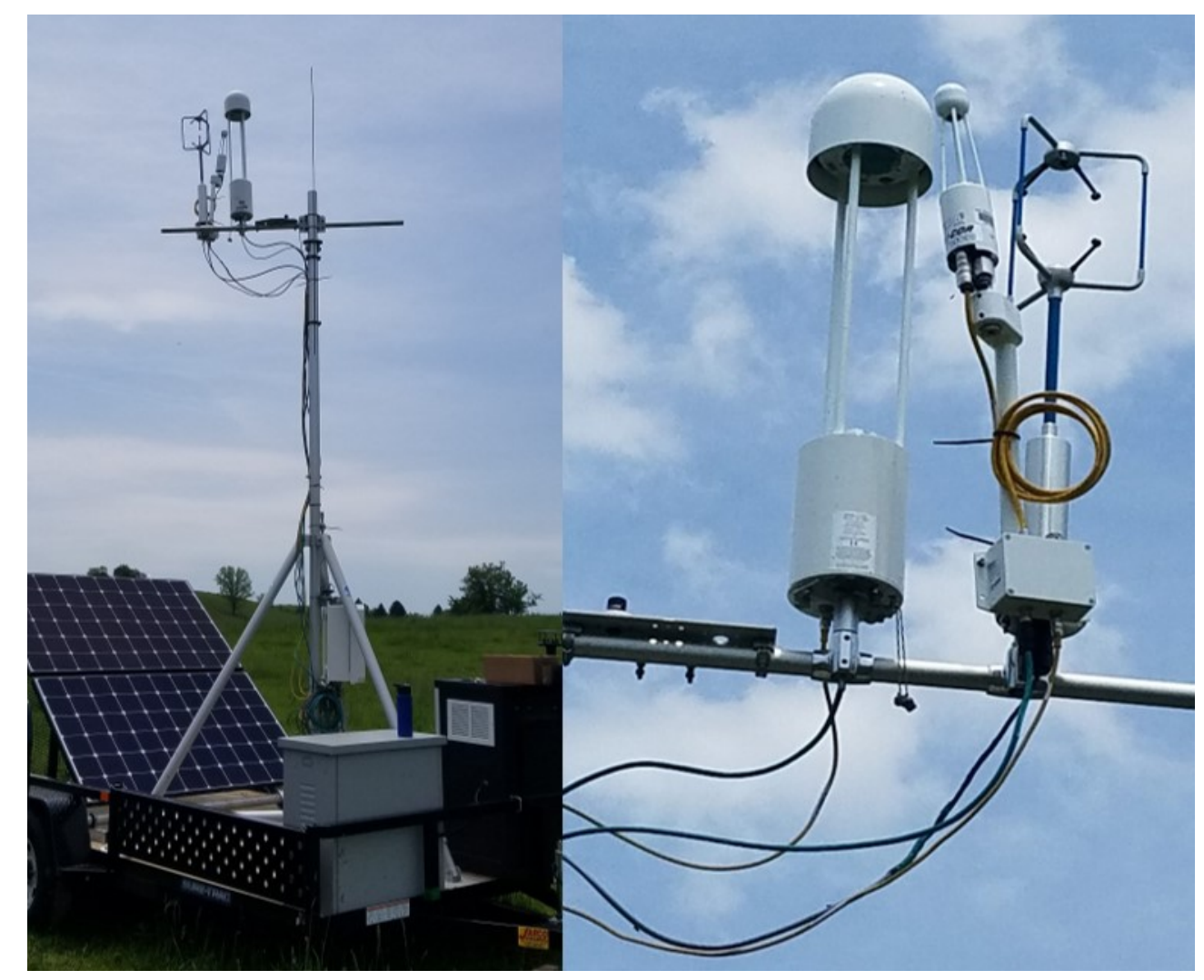

Figure 11. MECT deployed during controlled release experiments. 
The LI-7700 is a state-of-the-art methane analyzer which utilizes Wavelength Modulation Spectroscopy (WMS) across an open-path Herriott cell. It has a $0.5 \mathrm{~m}$ physical path length which translates to a full laser path length of approximately $30 \mathrm{~m}$. The instrument measures methane concentrations from 0 to $40 \mathrm{ppm}$ at $10 \mathrm{~Hz}$ with a resolution of $5 \mathrm{ppb}$. The analyzer can operate effectively in temperatures ranging from -25 to $50^{\circ} \mathrm{C}$ and pressures ranging from 50 to $110 \mathrm{kPa}$. The analyzer records these variables in addition to methane mole fraction for correct compensation. More details on how temperature and pressure affect the operating principles are available from LI-COR [108].

The LI-7500DS is an open-path $\mathrm{CO}_{2}$ and $\mathrm{H}_{2} \mathrm{O}$ analyzer which utilizes non-dispersive spectroscopy. The length of the optical path is $12.5 \mathrm{~cm}$. The instrument is capable of measuring $\mathrm{CO}_{2}$ molar density from 0 to $3000 \mu \mathrm{mol} / \mathrm{mol}$ and $\mathrm{H}_{2} \mathrm{O}$ molar density from 0 to $60 \mathrm{mmol} / \mathrm{mol}$ at a frequency of up to $10 \mathrm{~Hz}$. The measurement accuracy for both gases is within $1 \%$ of reading. The instrument also measures air temperature and pressure from -20 to $70^{\circ} \mathrm{C}$ and 50 to $110 \mathrm{kPa}$, respectively [109].

The LI-200R is a pyranometer designed to measure solar loading using a high stability silicon photovoltaic detector. The detector can measure solar loading up to $3000 \mathrm{~W} / \mathrm{m}^{2}$ with $\pm 3 \%$ typical uncertainty and maximum uncertainty of $\pm 5 \%$. It is calibrated against an Eppley Precision Spectral Pyranometer and gives an analog response of $75 \mu \mathrm{A}$ per 1000 watts per square meter $\left(\mathrm{W} / \mathrm{m}^{2}\right)$ [110]. The pyranometer output was recorded at a frequency of $10 \mathrm{~Hz}$, however, the recording method only allowed for $1 \mathrm{~Hz}$ granularity.

The Gill ${ }^{\circledR}$ WindMaster is a 3-axis sonic anemometer capable of measuring wind speed from 0 to $50 \mathrm{~m} / \mathrm{s}$ in three directions: $\mathrm{U}, \mathrm{V}$, and $\mathrm{W}$ corresponding to the $\mathrm{X}, \mathrm{Y}$, and $\mathrm{Z}$ directions in a standard rectangular coordinate system. The instrument has a resolution of $0.01 \mathrm{~m} / \mathrm{s}$. The accuracy is $<1.5 \%$ of the root mean square (RMS) value at $12 \mathrm{~m} / \mathrm{s}$. It also measures sonic temperature ranging from -40 to $70^{\circ} \mathrm{C}$ [111].

The Omega iBTHX is used to measure ambient variables such as temperature, pressure, relative humidity (RH), and dewpoint temperature. The Omega iBTHX measures these variables in rotary order, outputting the value of each variable once every 4 seconds. The barometric pressure is measured in the range of 0 to 1100 mbar with an accuracy of \pm 2 mbar and a resolution of 0.1 mbar. The relative humidity sensor is capable of measuring RH from 0 to $100 \%$ with an accuracy of $\pm 2 \%$ from 10 to $90 \%$ and $\pm 3 \%$ from 0 to $10 \%$ and 90 to $100 \%$. The instrument measured temperatures from 0 to $70^{\circ} \mathrm{C}$ with an accuracy of $\pm 0.8^{\circ} \mathrm{C}$ at $20^{\circ} \mathrm{C}$. The dewpoint temperature is reported in ${ }^{\circ} \mathrm{C}$ and is derived from the other variables [112]. The variables recorded by the Omega $\mathrm{iBTHX}$ were not directly used in calculations but were used for data sorting and verification.

All data were recorded using a BeagleBone Black [113]. The BeagleBone utilized a Linux based operating system which contain the in-house data collection software known as Scimitar, which was developed in Python 2.7 [114]. The BeagleBone was housed in the control box and powered by a $5 \mathrm{~V}$ power supply. All data were stored on an SD card and transferred to a computer every 2 weeks. A DIGI TransPort ${ }^{\circledR}$ WR11 $\mathrm{XT}$ was connected to the BeagleBone to provide a mobile $3 \mathrm{G}$ signal [115]. A Hologram IoT SIM card was installed in the WR11 XT and acted as the service provider [116]. This allowed the BeagleBone to maintain accurate time and date and allowed for alerts to be sent via email if data collection was halted for any reason.

The LI-7700, LI-7500, and WindMaster were mounted vertically, $4 \mathrm{~m}$ from the base of the trailer. The trailer base was about $0.5 \mathrm{~m}$ above the ground; therefore, the sensor height was considered $4.5 \mathrm{~m}$ for data processing. LI-200R was mounted on a flat plate and positioned on a horizontal bar to capture solar loading. The Omega iBTHx was mounted to the DAQ box about $1 \mathrm{~m}$ from the base of the trailer. Another aspect of the system that is considered important for EC data processing is sensor separation. It is desirable during EC measurements that the instruments of importance (the device measuring the gas of interest, and the sonic anemometer) have a minimal amount of interference and space between them. The separations between instruments are necessary to estimate high-frequency flux losses [117]. Three separation measurements were required as inputs to the EC post processing software EddyPro ${ }^{\circledR}$. These were 
"northward", "eastward", and "vertical". The center of the anemometer is considered the origin and these separations are measured in three directions from that point to the center of the corresponding analyzer. The setups were different during the two campaigns, which was not ideal, however, the post processing software takes the separations into account when calculating fluxes. The sensor separations from each campaign are presented in Table 18 and displayed in Figure 12.

Table 18. MECT sensor separation distances during campaigns.

\begin{tabular}{|c|c|c|c|c|c|}
\hline \multicolumn{2}{|c|}{ Location } & \multicolumn{2}{c|}{ Farm } & \multicolumn{2}{c|}{ MSEEL } \\
\hline \multicolumn{2}{|c|}{ Instrument } & LI-7700 & LI-7500 & LI-7700 & LI-7500 \\
\hline \multirow{3}{*}{$\begin{array}{c}\text { Separation } \\
(\mathrm{cm})\end{array}$} & Northward & 12.7 & -17.8 & 2.5 & 21.0 \\
\cline { 2 - 6 } & Eastward & -19.1 & -20.3 & -24.4 & -7.6 \\
\cline { 2 - 6 } & Vertical & -12.7 & 0 & -16.5 & 0 \\
\hline
\end{tabular}

The entire system was powered by two LG NeON ${ }^{\circledR} \mathrm{R} 360$-watt solar panels connected to two 12-volt Sun Xtender PVX-2120L solar batteries through a TriStar MPPTTM-60 solar battery charger [118]-[120]. The solar system worked as designed and during the entirety of data collection, power was only lost once due to lack of power during February of 2020, resulting in five days of system downtime. A complete system diagram is presented in Figure 13 and wiring details are presented in Figure 14. 
Table 19. Summary of major MECT equipment and their specifications [108]-[112].

\begin{tabular}{|c|c|c|c|c|c|c|c|}
\hline Device & Manufacturer & $\begin{array}{l}\text { Detection } \\
\text { Method }\end{array}$ & $\begin{array}{l}\text { Max } \\
\text { Rate/ } \\
\text { Used } \\
\text { Rate }\end{array}$ & $\begin{array}{l}\text { Parameters } \\
\text { Measured }\end{array}$ & Range & $\begin{array}{l}\text { Resolution (res) } \\
\text { /Accuracy (acc) }\end{array}$ & Operating Limits \\
\hline \multirow{2}{*}{$\begin{array}{c}\text { Gill } \\
\text { WindMaster }\end{array}$} & \multirow{2}{*}{$\begin{array}{l}\text { Gill Instruments } \\
\text { Ltd. }\end{array}$} & \multirow{2}{*}{$\begin{array}{l}\text { Ultrasonic } \\
\text { Pulse }\end{array}$} & \multirow{2}{*}{$\begin{array}{c}20 \mathrm{~Hz} / \\
10 \mathrm{~Hz}\end{array}$} & \multirow{2}{*}{$\begin{array}{l}\text { 3-D Wind } \\
\text { Speed }\end{array}$} & \multirow{2}{*}{$0-50 \mathrm{~m} / \mathrm{s}$} & \multirow{2}{*}{$<1.5 \% \mathrm{RMS}$} & $\mathbf{T}:-40-70^{\circ} \mathrm{C}$ \\
\hline & & & & & & & $\mathbf{R H}:<5-100 \%$ \\
\hline \multirow{3}{*}{ LI-7700 } & \multirow{3}{*}{$\begin{array}{c}\text { LI-COR } \\
\text { Biosciences }\end{array}$} & \multirow{3}{*}{$\begin{array}{l}\text { Wavelength } \\
\text { Modulation } \\
\text { Spectroscopy }\end{array}$} & \multirow{3}{*}{$\begin{array}{c}20 \mathrm{~Hz} / \\
10 \mathrm{~Hz}\end{array}$} & \multirow{3}{*}{$\begin{array}{c}\mathrm{CH}_{4} \text { conc. } \\
\text { Temperature } \\
\text { Pressure }\end{array}$} & \multirow{3}{*}{$\begin{array}{l}\mathbf{C H}_{4}: 0-40 \\
\text { ppm at } 25^{\circ} \mathrm{C}\end{array}$} & \multirow{3}{*}{$\begin{array}{c}5 \text { ppb res. } \\
<1 \% \text { linearity }\end{array}$} & $\mathbf{T}:-25-50^{\circ} \mathrm{C}$ \\
\hline & & & & & & & P: $50-110 \mathrm{kPa}$ \\
\hline & & & & & & & RH: 0 - 100\% \\
\hline \multirow{4}{*}{ LI-7500 } & \multirow{4}{*}{$\begin{array}{c}\text { LI-COR } \\
\text { Biosciences }\end{array}$} & \multirow{4}{*}{$\begin{array}{l}\text { Non-dispersive } \\
\text { spectroscopy }\end{array}$} & \multirow{4}{*}{$\begin{array}{c}20 \mathrm{~Hz} / \\
10 \mathrm{~Hz}\end{array}$} & \multirow{4}{*}{$\begin{array}{c}\mathrm{CO}_{2} \text { conc. } \\
\mathrm{H}_{2} \mathrm{O} \text { conc. } \\
\text { Temperature } \\
\text { Pressure }\end{array}$} & $\begin{array}{c}\mathrm{CO}_{2}: 0-3000 \\
\mu \mathrm{mol} / \mathrm{mol}\end{array}$ & $\mathbf{C O}_{2}:<1 \%$ of reading & \multirow{2}{*}{ RH: 0 - 95\% } \\
\hline & & & & & $\begin{array}{c}\mathbf{H}_{2} \mathbf{O}: 0-60 \\
\mu \mathrm{mol} / \mathrm{mol} \\
\end{array}$ & $\mathbf{H}_{2} \mathbf{O}:<1 \%$ of reading & \\
\hline & & & & & T: $-20-70^{\circ} \mathrm{C}$ & $\mathbf{T}:+-0.3^{\circ} \mathrm{C}$ & T: $-25-50^{\circ} \mathrm{C}$ \\
\hline & & & & & P: $50-110 \mathrm{kPa}$ & P: $0.4 \mathrm{kPa}$ & P: $50-110 \mathrm{kPa}$ \\
\hline \multirow{2}{*}{ LI-200R } & \multirow{2}{*}{$\begin{array}{c}\text { LI-COR } \\
\text { Biosciences }\end{array}$} & \multirow{2}{*}{ Photovoltaic } & \multirow{2}{*}{$\begin{array}{c}1 \mathrm{e} 5 \mathrm{~Hz} / \\
10 \mathrm{~Hz}\end{array}$} & \multirow{2}{*}{ Solar Loading } & \multirow{2}{*}{$0-3000 \mathrm{~W} / \mathrm{m}^{2}$} & \multirow{2}{*}{ $\pm 3 \%$ over reading } & $\mathbf{T}:-40-65^{\circ} \mathrm{C}$ \\
\hline & & & & & & & RH: 0 - 100\% \\
\hline \multirow{3}{*}{$\begin{array}{l}\text { Omega } \\
\text { iBTHx }\end{array}$} & \multirow{3}{*}{$\begin{array}{l}\text { Omega }^{\mathrm{TM}} \\
\text { Engineering }\end{array}$} & \multirow{3}{*}{ Various } & \multirow{3}{*}{$\begin{array}{l}0.25 \mathrm{~Hz} / \\
0.25 \mathrm{~Hz}\end{array}$} & \multirow{3}{*}{$\begin{array}{c}\text { Temperature } \\
\text { Pressure } \\
\text { Relative } \\
\text { Humidity }\end{array}$} & $\mathbf{T}: 0-70^{\circ} \mathrm{C}$ & $\begin{array}{c}\mathbf{T}: \pm 2^{\circ} \mathrm{C} \text { acc. } \\
0.01^{\circ} \mathrm{C} \text { res. }\end{array}$ & $\mathbf{T}: 0-70^{\circ} \mathrm{C}$ \\
\hline & & & & & $\mathbf{P}: 0-110 \mathrm{kPa}$ & $\begin{array}{c}\mathbf{P}: \pm 0.2 \mathrm{kPa} \text { acc. } \\
0.01 \mathrm{kPa} \text { res. }\end{array}$ & $\mathbf{P}: 0-110 \mathrm{kPa}$ \\
\hline & & & & & RH: 0 - 100\% & $\begin{array}{c}\text { RH: } 2 \% \text { for } 10-90 \text { acc. } \\
0.03 \% \text { res. }\end{array}$ & RH: $0-100 \%$ \\
\hline
\end{tabular}




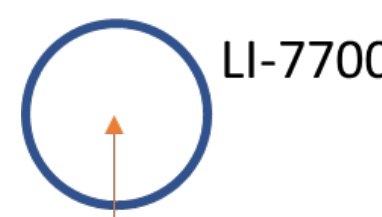

\section{Farm}

12.7

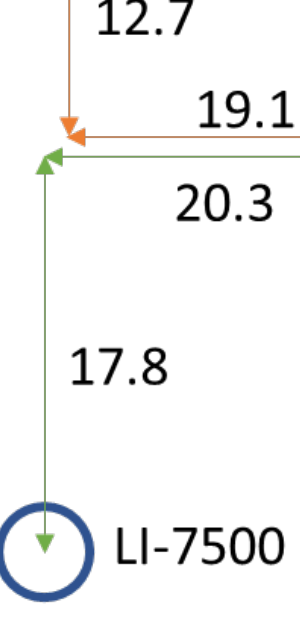

\section{MSEEL}

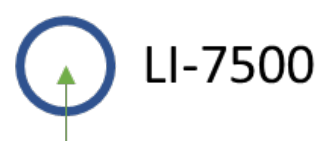

21.0

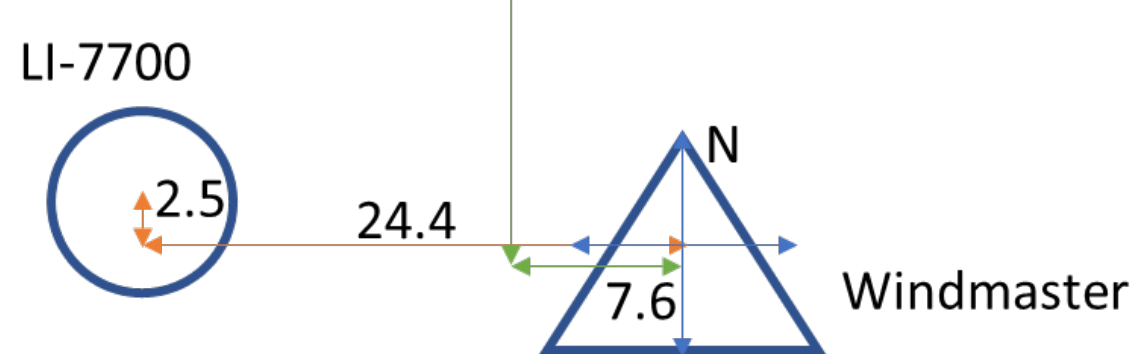

Figure 12. MECT sensor separation schematic during data collection campaigns. 


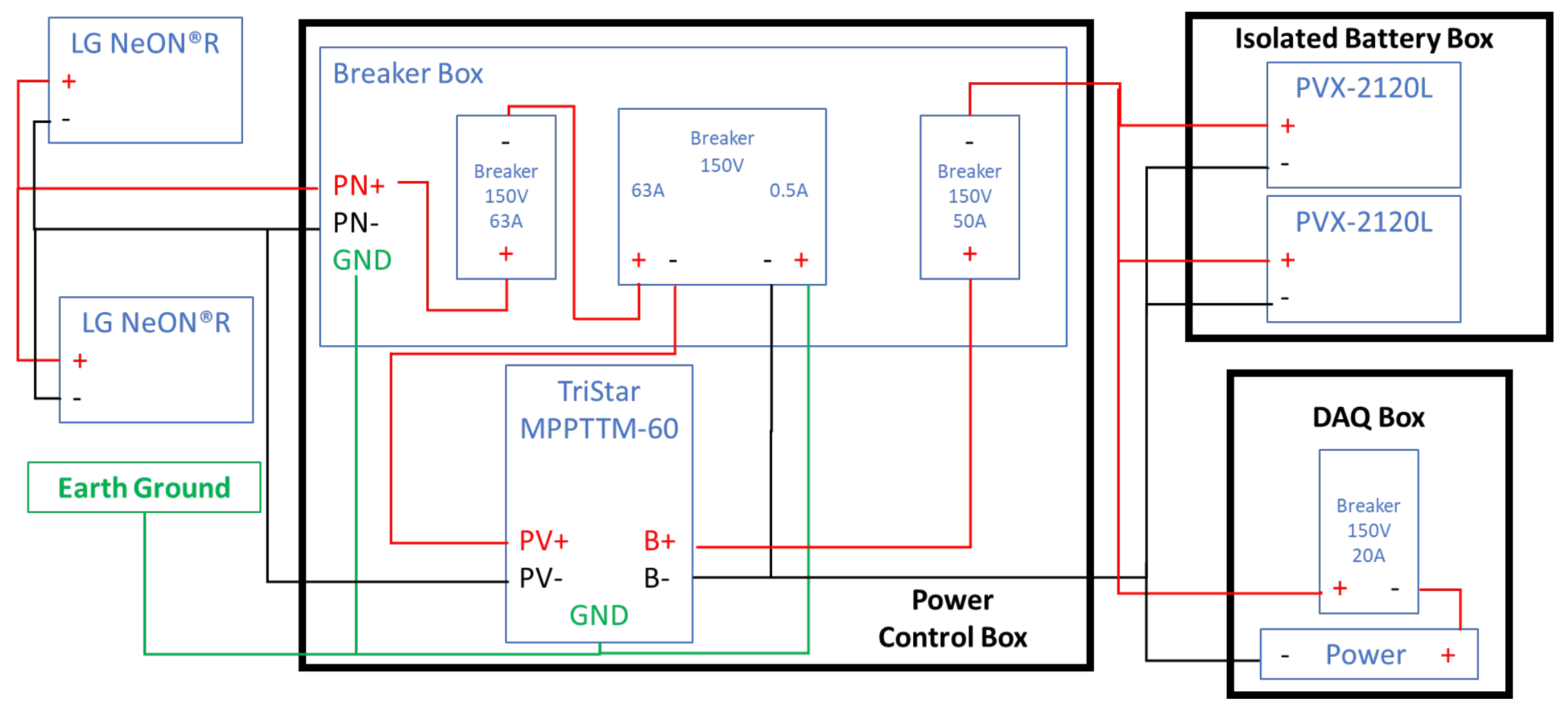

Figure 13. Wiring diagram for MECT power system. 


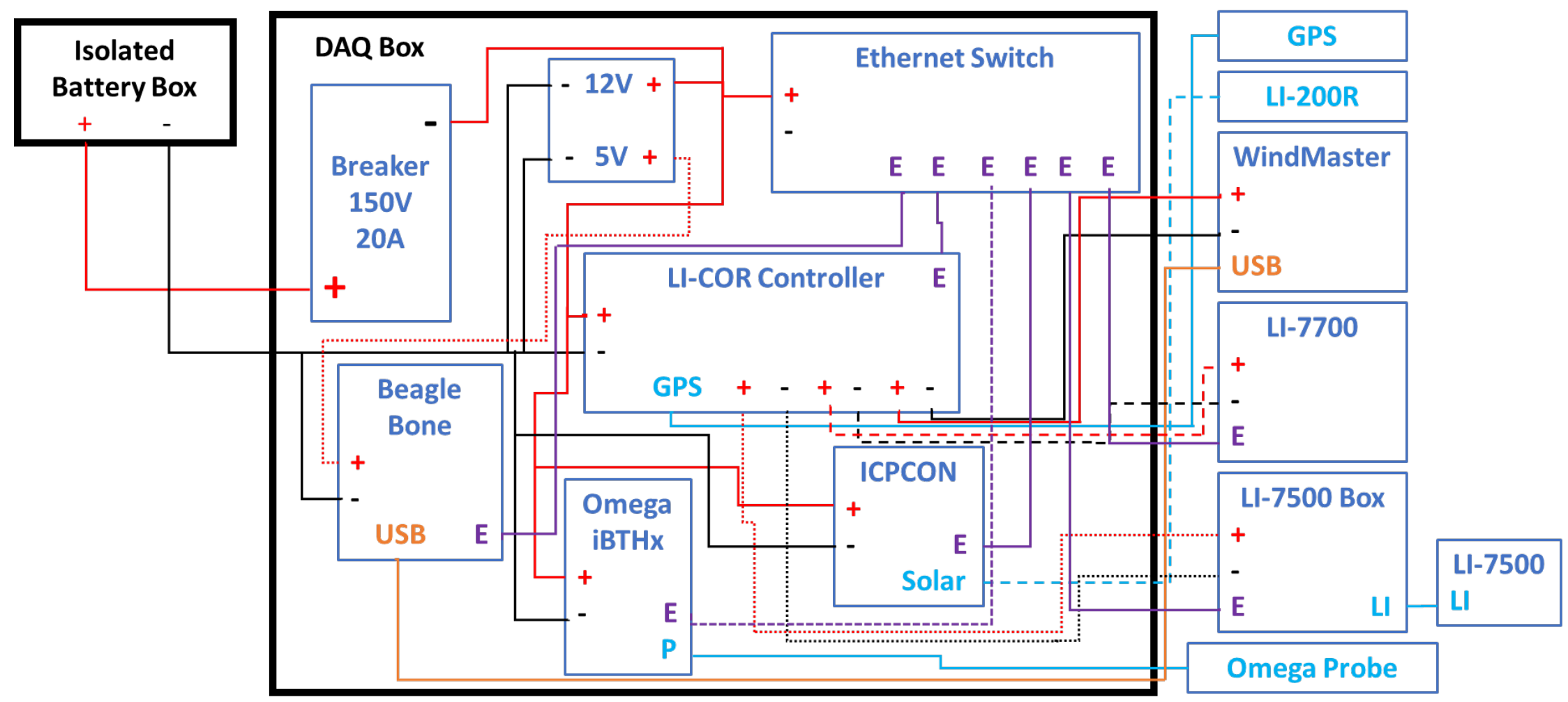

Figure 14. Wiring diagram of the MECT data acquisition system. 


\subsubsection{Full Flow Sampling System (FFS)}

The primary means of direct methane quantification was the WVU developed FFS system, presented in Figure 15. The FFS is a system consisting of an explosion proof blower, sampling hose, thermocouple, mass airflow sensor (MAF), and an emissions analyzer. The FFS operates on the same principle as dilution tunnels for engine emissions certification in which a sample (in this case NG emissions) is diluted with background air and the concentration of a specific gas (in this case methane) is measured. During operation, the diluted sample is pulled through the sampling hose by the blower, which then pushes the sample through a pipe with a length of at least ten diameters to ensure adequate mixing. At the end of the pipe is a MAF which measures the total volume of dilute gas sample passing through the system. Once the dilute gas passes through the MAF a sample is pulled through an emissions analyzer to measure the composition. The temperature of the dilute gas is also measured for correction to standard flow rate. A detailed description of operation is presented by Johnson et al [107]. All data were recorded at a rate of at least $1 \mathrm{~Hz}$ with the WVU in-house developed software, Scimitar, which is open source and allows easy integration with multiple devices. To calculate methane mass rate emissions from the concentration and flow rate the following equations were used.

$$
\begin{aligned}
\mathrm{CH}_{4}(g / \mathrm{hr})= & \left(\frac{p p m}{1000000}\right) *\left(\text { flow }\left(\frac{f t^{3}}{\mathrm{~min}}\right)\right) *\left(28.3 \frac{\mathrm{L}}{f t^{3}}\right) \\
& *\left(16.04 \frac{\mathrm{g}}{\mathrm{mole}}\right) *\left(\frac{\mathrm{mol}}{\mathrm{L}}\right) *\left(60 \frac{\mathrm{min}}{\mathrm{hr}}\right) \\
\frac{\mathrm{mol}}{L} & =\left(\frac{P(\mathrm{kPa})}{T(\mathrm{~K}) * 8.314\left(\frac{\mathrm{Nm}}{\mathrm{mol} \mathrm{K}}\right)}\right)
\end{aligned}
$$

Equation 13

Equation 14

Two versions of the FFS were deployed during the direct quantification audits conducted. All components and operation remained the same, except for the analyzer used for measurement. For audits \#1 through \#11 a Los Gatos Research (LGR) Ultra-Portable Greenhouse Gas (UGGA) was used as the methane analyzer in the system [121]. After November of 2019 (audits 12 through 17) the system was upgraded with a LGR Fast Methane-Ethane Analyzer (FMEA) [122]. These audits corresponded to those when the MECT was deployed at the MSEEL site. The use of the FMEA allowed for the measurement of ethane which is often used to correlate atmospheric methane concentrations with those from NG rather than natural systems. Methane/ethane ratios are also relevant to the quality of natural gas extracted. The specifications of both analyzers are presented in Table 20. 

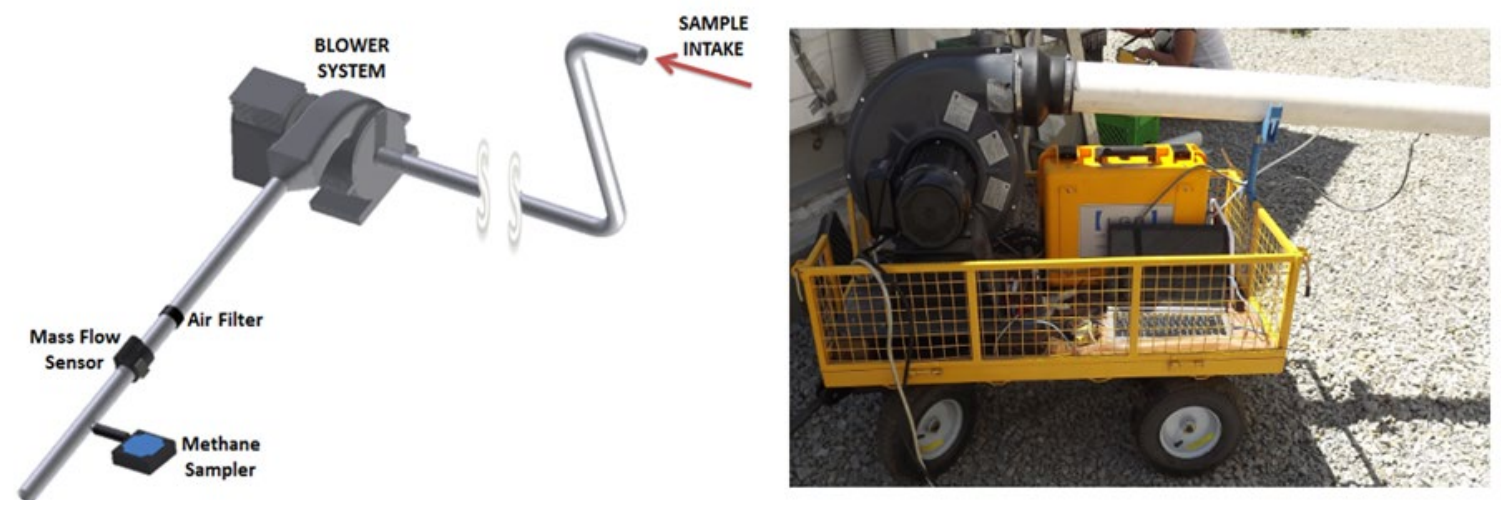

Figure 15. FFS schematic (left) and in use with UGGA (right).

Table 20. FFS methane analyzer specifications [121], [122].

\begin{tabular}{|c|c|c|}
\hline Analyzer & $U G G A$ & FMEA \\
\hline \multicolumn{3}{|c|}{ Measurement Range } \\
\hline $\mathrm{CH}_{4}(\mathrm{ppm})$ & $0.01-100$ & $1-1000$ \\
\hline $\mathrm{C}_{2} \mathrm{H}_{6}(\mathrm{ppm})$ & - & $0-15$ \\
\hline $\mathrm{CO}_{2}(\mathrm{ppm})$ & $1-20000$ & - \\
\hline $\mathrm{H}_{2} \mathrm{O}(\mathrm{ppm})$ & $500-70000$ & $5000-25000$ \\
\hline \multicolumn{3}{|c|}{ Operational Range } \\
\hline $\mathrm{CH}_{4}(\mathrm{ppm})$ & $0-100000^{*}$ & $0-10000$ \\
\hline $\mathrm{C}_{2} \mathrm{H}_{6}(\mathrm{ppm})$ & - & $0-1000$ \\
\hline $\mathrm{CO}_{2}(\mathrm{ppm})$ & $0-20000$ & - \\
\hline $\mathrm{H}_{2} \mathrm{O}(\mathrm{ppm})$ & $0-70000$ & $0-70000$ \\
\hline \multicolumn{3}{|c|}{ Repeatability/Precision } \\
\hline $\mathrm{CH}_{4}(1 \sigma, 1 \mathrm{~s})$ & $<2 \mathrm{ppb}$ & $<2 \mathrm{ppb}$ \\
\hline $\mathrm{C}_{2} \mathrm{H}_{6}(1 \sigma, 1 \mathrm{~s})$ & - & $<30 \mathrm{ppb}$ \\
\hline $\mathrm{CO}_{2}(1 \sigma, 1 \mathrm{~s})$ & $<300 \mathrm{ppb}$ & - \\
\hline $\mathrm{H}_{2} \mathrm{O}(1 \sigma, 1 \mathrm{~s})$ & $<100 \mathrm{ppm}$ & $<20 \mathrm{ppm}$ \\
\hline \multicolumn{3}{|c|}{ Physical Limits } \\
\hline Cell Volume (cc) & 343 & 355 \\
\hline Cell Pressure (Torr) & 140 & 500 \\
\hline Operating Temperature $\left({ }^{\circ} \mathrm{C}\right)$ & $5-45$ & $5-50$ \\
\hline
\end{tabular}

*high range model (utilized for audits)

To verify the accuracy of the FFS system, calibrations of the analyzers and MAF were performed semiannually. In addition, spot checks were performed before each audit. These checks typically involved verification of the analyzer on 1 to 10 different concentrations of methane. An example of a 10-point calibration checks on the FMEA is presented in Figure 16. 


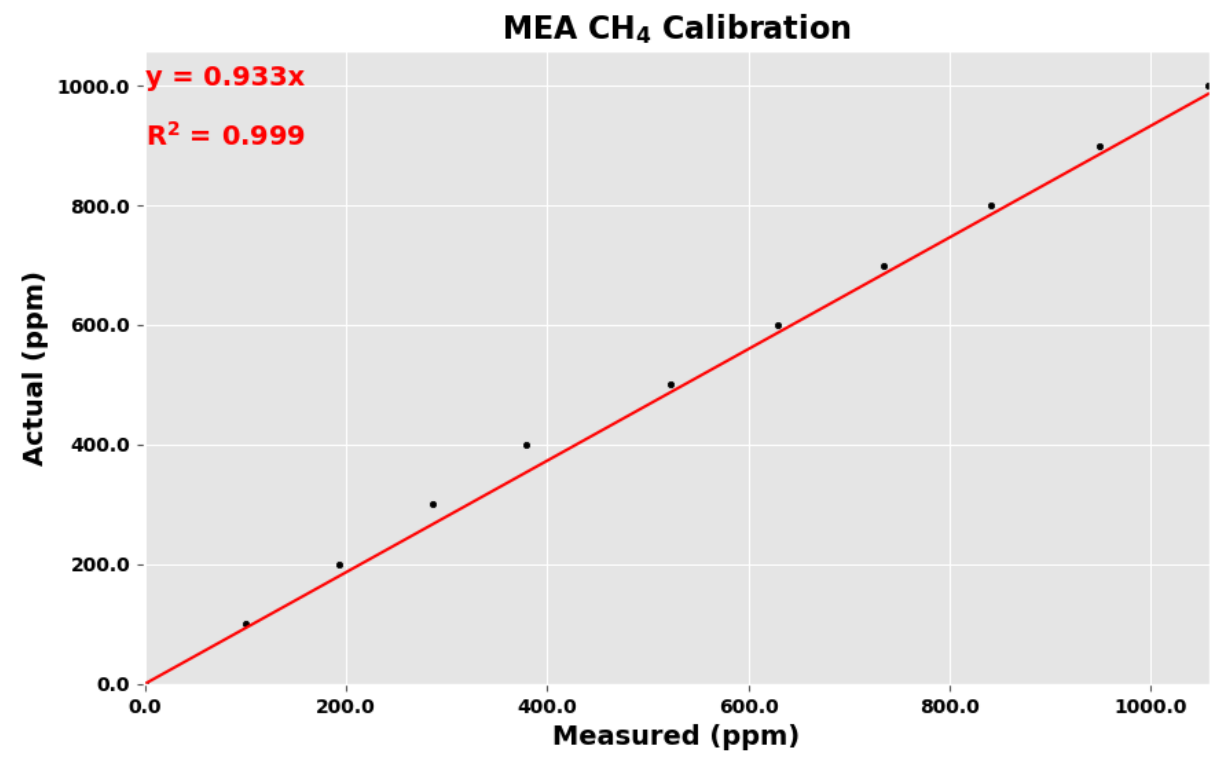

Figure 16. Example of FMEA methane calibration.

In 2018 a complete in-depth system calibration was also performed, including calibrations of two UGGAs and MAF, as well as recovery tests. The recovery tests were performed with a known release of methane mass emissions. The tests were performed with $99.9 \%$ methane and mass flow controllers. The operators of the FFS were not informed of the actual rate and had to calculate mass rate emissions to ensure that the system was accurate. The results of the recovery tests verified the accuracy of the FFS across a range of mass emissions rates from 1 gram per hour $(\mathrm{g} / \mathrm{hr})$ to 1 kilogram per hour $(\mathrm{kg} / \mathrm{hr})$. The recovery test data is presented in Table 21.

Table 21. FFS system results from various mass emission recovery tests.

\begin{tabular}{|c|c|c|c|c|c|c|c|c|}
\hline \multirow{2}{*}{$\begin{array}{c}\text { Setpoint } \\
(\mathbf{g} / \mathbf{h r})\end{array}$} & \multicolumn{2}{|c|}{ Reported Value 1 } & \multicolumn{2}{c|}{ Reported Value 2 } & \multicolumn{2}{c|}{ Reported Value 3 } & \multicolumn{2}{c|}{ Average Value } \\
\cline { 2 - 9 } & $\begin{array}{c}\text { Estimate } \\
(\mathbf{g} / \mathbf{h r})\end{array}$ & $\begin{array}{c}\text { Error } \\
\mathbf{( \% )}\end{array}$ & $\begin{array}{c}\text { Estimate } \\
\mathbf{( g / h r})\end{array}$ & $\begin{array}{c}\text { Error } \\
\mathbf{( \% )}\end{array}$ & $\begin{array}{c}\text { Estimate } \\
\mathbf{( g / h r})\end{array}$ & $\begin{array}{c}\text { Error } \\
\mathbf{( \% )}\end{array}$ & $\begin{array}{c}\text { Estimate } \\
(\mathbf{g} / \mathbf{h r})\end{array}$ & $\begin{array}{c}\text { Error } \\
\mathbf{( \% )}\end{array}$ \\
\hline 1.002 & 1.002 & 0 & 0.987 & -1.5 & 0.951 & -5.1 & 0.980 & -2.2 \\
\hline 10.01 & 10.03 & 0.2 & 9.94 & -0.7 & 10.09 & 0.8 & 10.02 & 0.1 \\
\hline 200.65 & 195.6 & -2.5 & 193.4 & -3.6 & 196.5 & -2.1 & 195.2 & -2.7 \\
\hline 1000 & 1001.8 & 0.2 & 990.5 & -1 & 1012.0 & 1.2 & 1001.4 & 0.1 \\
\hline
\end{tabular}

The MAF used in the system is a standard General Motors Original Equipment Mass Airflow Sensor. Its output range is $0-5$ volts [123]. Typically, calibrations of the MAF were performed semi-annually against a National Institute of Standards and Technology (NIST) traceable laminar flow element (LFE) from Meriam ${ }^{\circledR}$ [124]. The calibrations were performed on a flow-bench at the WVU Engine Research Center (ERC). An example of a MAF calibration against the LFE using a 24-point check is presented in Figure 17. 


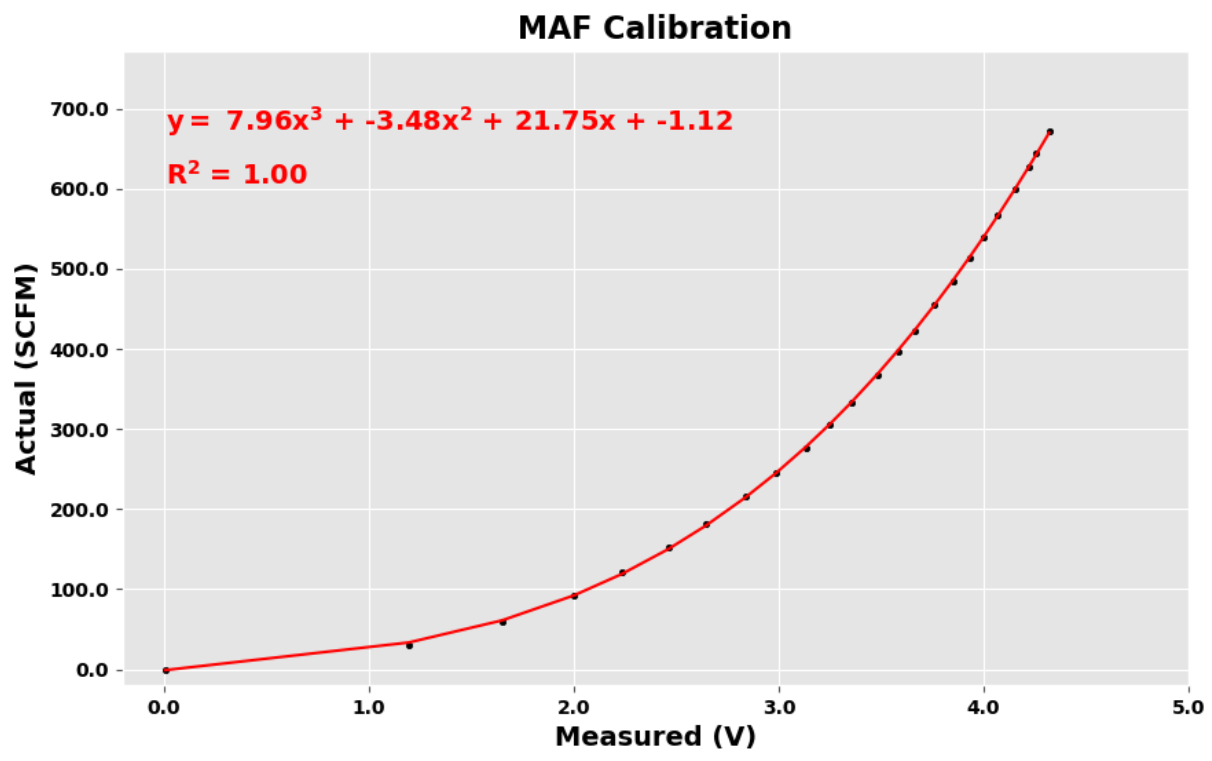

Figure 17. Example of MAF calibration against NIST traceable LFE.

\subsubsection{Marcellus Shale Energy and Environment Laboratory (MSEEL) Production Site}

The MSEEL is a production site located in Westover, WV. It contains four active wells which provides NG to the greater Morgantown, WV area. The project supporting the completion of the wells was sponsored by the DOE's National Energy Technology Laboratory (NETL) in collaboration with WVU, Northeast Natural Energy, and Schlumberger. It was designed to "provide a long-term field site to develop and validate new knowledge and technology to improve recovery efficiency and minimize environmental implications of unconventional resource development" [106]. Drilling of the site began in 2011 with completion of the last horizontal well occurring in December of 2015. The site contains four active wells (MIP-3H, MIP-4H, MIP-5H, and MIP-6H). Two of these wells MIP-4H and MIP-6H have been active since December 2011 while the other two wells came online in December of 2015. Since then, all four wells have been in-line for different amounts of time depending on the energy demand of the area. The gas and water production of the site are presented in Figure 18 and Figure 19, respectively. 


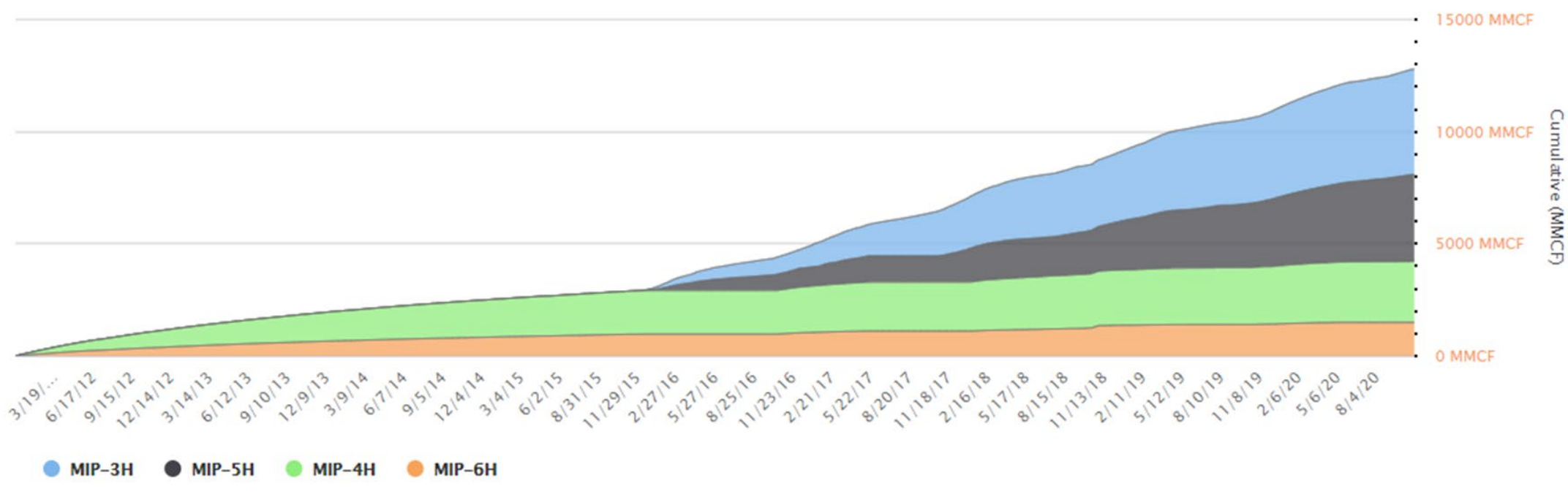

Figure 18. MSEEL cumulative gas production [106].

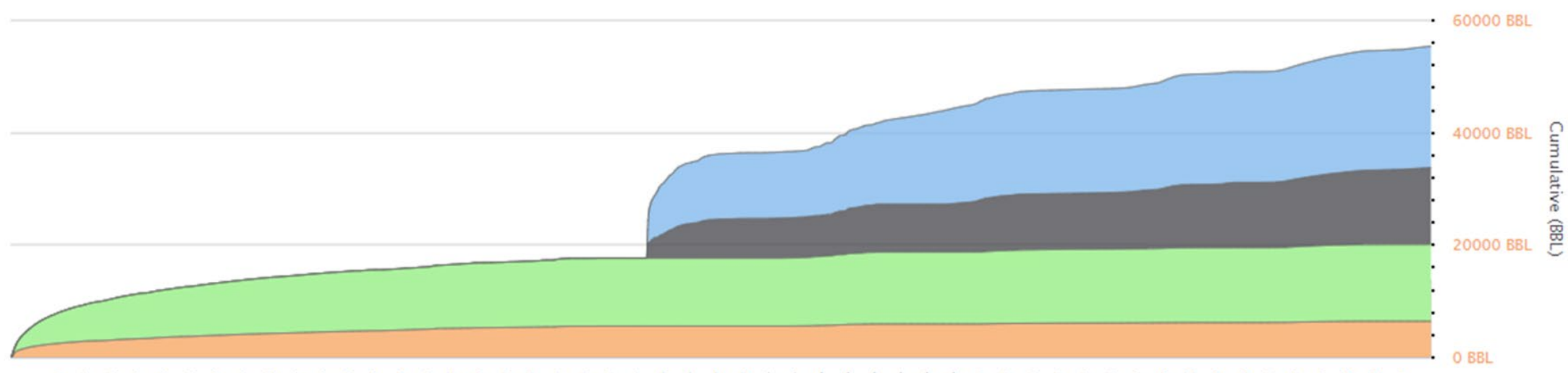

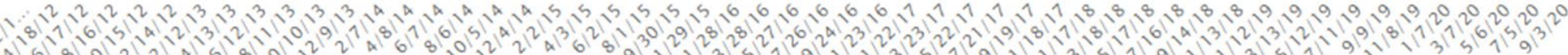

MIP-3H MIP-5H MIP-4H MIP-6H

Figure 19. MSEEL cumulative water production [106]. 
In addition to the four producing wellheads, MSEEL also contains equipment for initial handling and processing of the gas and water produced. This equipment includes a produced water tank, two enclosed GPUs (EGPUs), two production units, two vertical sand separators, a thermoelectric generator, and other associated piping and equipment. All components described are considered sources of potential leaks. An image of the equipment typically on-site is presented in Figure 20. The estimated total area of the pad is just over $1.1 \times 10^{4} \mathrm{~m}^{2}$. The dimensions and layout of the site are presented in Figure 21 and Table 22. The layout and size of the site were expected to impact the deployment of the MECT on site.

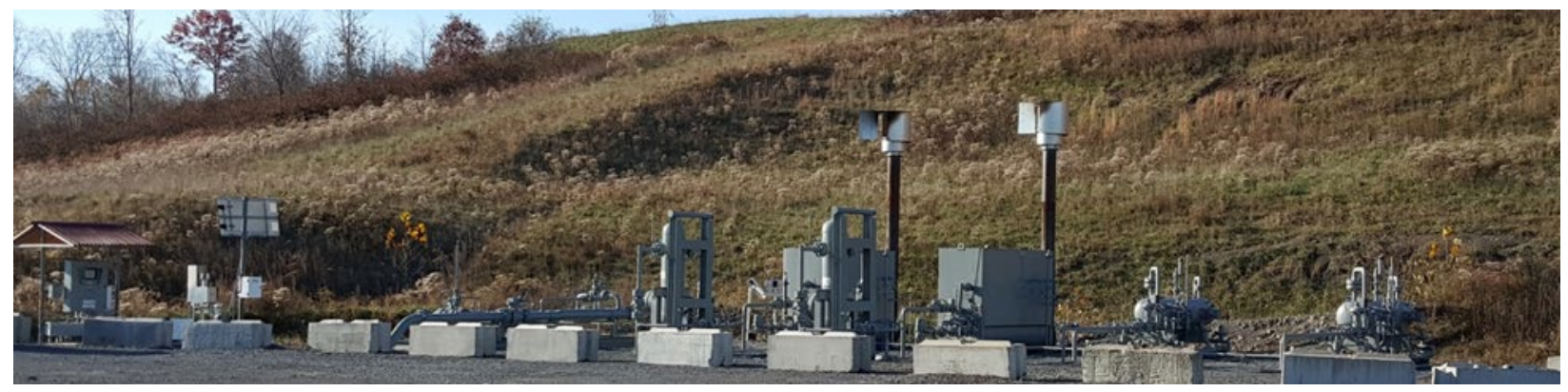

Figure 20. Processing equipment at the MSEEL site.

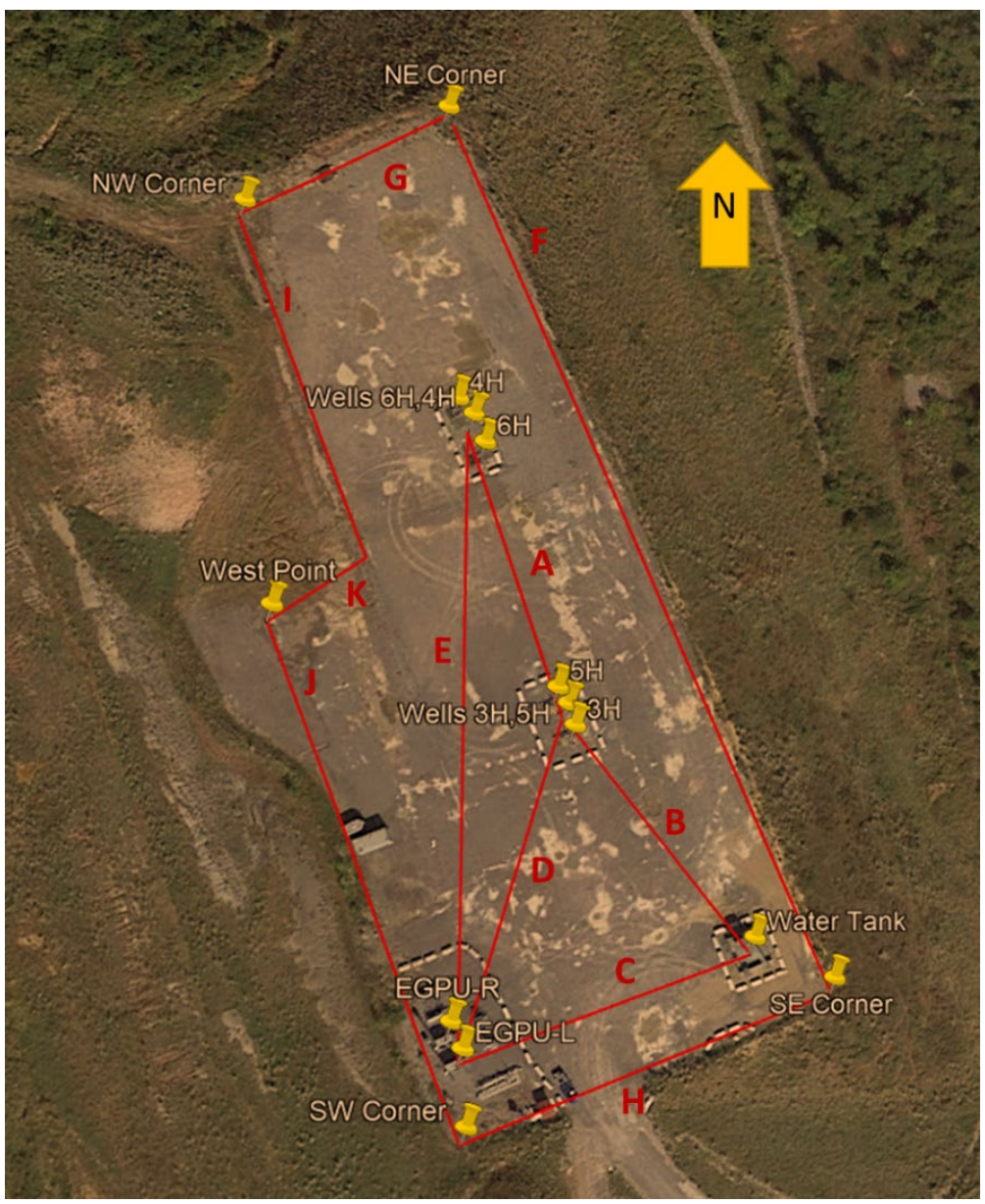

Figure 21. Layout of the MSEEL site (view from Google Earth $\left.{ }^{\circledR}\right)$. 
Table 22. MSEEL dimensions measured with Google Earth ${ }^{\circledR}$

\begin{tabular}{|c|c|c|}
\hline Description & $\begin{array}{c}\text { Figure 21 } \\
\text { Label }\end{array}$ & $\begin{array}{c}\text { Distance } \\
(\mathbf{m})\end{array}$ \\
\hline Distance Between Wells & $\mathrm{A}$ & 55 \\
\hline Well35 to Water Tank & $\mathrm{B}$ & 54 \\
\hline EGPUs to Tank & $\mathrm{C}$ & 56 \\
\hline EGPUs to Well35 & $\mathrm{D}$ & 63 \\
\hline EGPUs to Well46 & $\mathrm{E}$ & 112 \\
\hline Full Length & $\mathrm{F}$ & 173 \\
\hline Thin Width & $\mathrm{G}$ & 42 \\
\hline Full Width & $\mathrm{H}$ & 72 \\
\hline Other Edge1 & $\mathrm{I}$ & 67 \\
\hline Other Edge2 & $\mathrm{J}$ & 98 \\
\hline Other Edge3 & $\mathrm{K}$ & 21 \\
\hline
\end{tabular}

The MSEEL production site was a real-world point of comparison for the indirect quantification methods employed by this research. To compare indirect quantification methods to real world emissions, direct quantification measurements were required. The FFS system was used to directly quantify methane emissions from the MSEEL site 17 times beginning in November of 2016. The emissions audits were performed quarterly over a four-year span concluding in November of 2020. The goal of these audits which were part of the MSEEL project funded by the DOE, was to assess the temporal variability of methane emissions from the site. These audits consisted of measuring all possible sources of methane emissions. The emissions from the methane audits serve as an order of magnitude and variability verification for the indirect quantification performed by the MECT. The dates of the performed audits are presented in Table 24, along with all emissions measurements recorded. For comparison to MECT estimates, there were 6 total audits performed during the deployment of the MECT. Successful MECT estimations were made during 5 of those audits. These audits, along with the historical audits provide insight into the scale of emissions expected from the site.

During every audit, each of the four wellheads was tented and the methane mass flux from the entire wellhead was averaged over a period of 15-30 minutes. A tented well is presented in the bottom of Figure 22. The dimensions of the tent were approximately $20 \mathrm{ft}$ by $10 \mathrm{ft}$. The tank emissions were measured based on the status of the produced water tank at that time. For example, if the thief hatch was open on the date of the audit it was measured by using a large plastic sampling bin at the end of the FFS sampling hose. The vent was sampled in a similar manner. An example of tank measurement is displayed in Figure 22 (top right). The EGPUs internal emissions were measured by inserting the sampling line into the opened door, holding it closed over the hose, and sampling until a steady state value was obtained. This allowed for some dilution air to enter the EGPU and prevented suction pressure. Other components on the site were sampled with the sampling hose held near the leak for a few minutes until a steady state value was obtained. No component was ever enclosed by the sampling method. This allowed sufficient dilution air and no suction pressure. Non-major components were checked for leaks using an Eagle 2 Gas Detector from RKI Instruments (Eagle 2) [125]. Any concentration reading seen above $100 \mathrm{ppm}$ was sampled with the FFS. An example of the Eagle 2 detecting a leak is presented in Figure 22 (top left). The leaks that were identified were measured and were often from the same components over the course of data collection. An example of leak measurement is presented in Figure 22 (top middle). Some examples of leaks from non-major components are presented in Figure 23. The same major measurements (four wellheads, tank, two EGPUs) were taken during all audits, with a few exceptions. There was only one EGPU on site during the July 2017 audit (\#3) and no leak measurements were taken due to lack of access to area, no EGPU measurements 
were recorded during the October 2018 audit (\#7) due to inability to access enclosures. The number of leaks measured varied across audits, but there were typically between four and ten leaks located using the Eagle 2 sampling technique. 


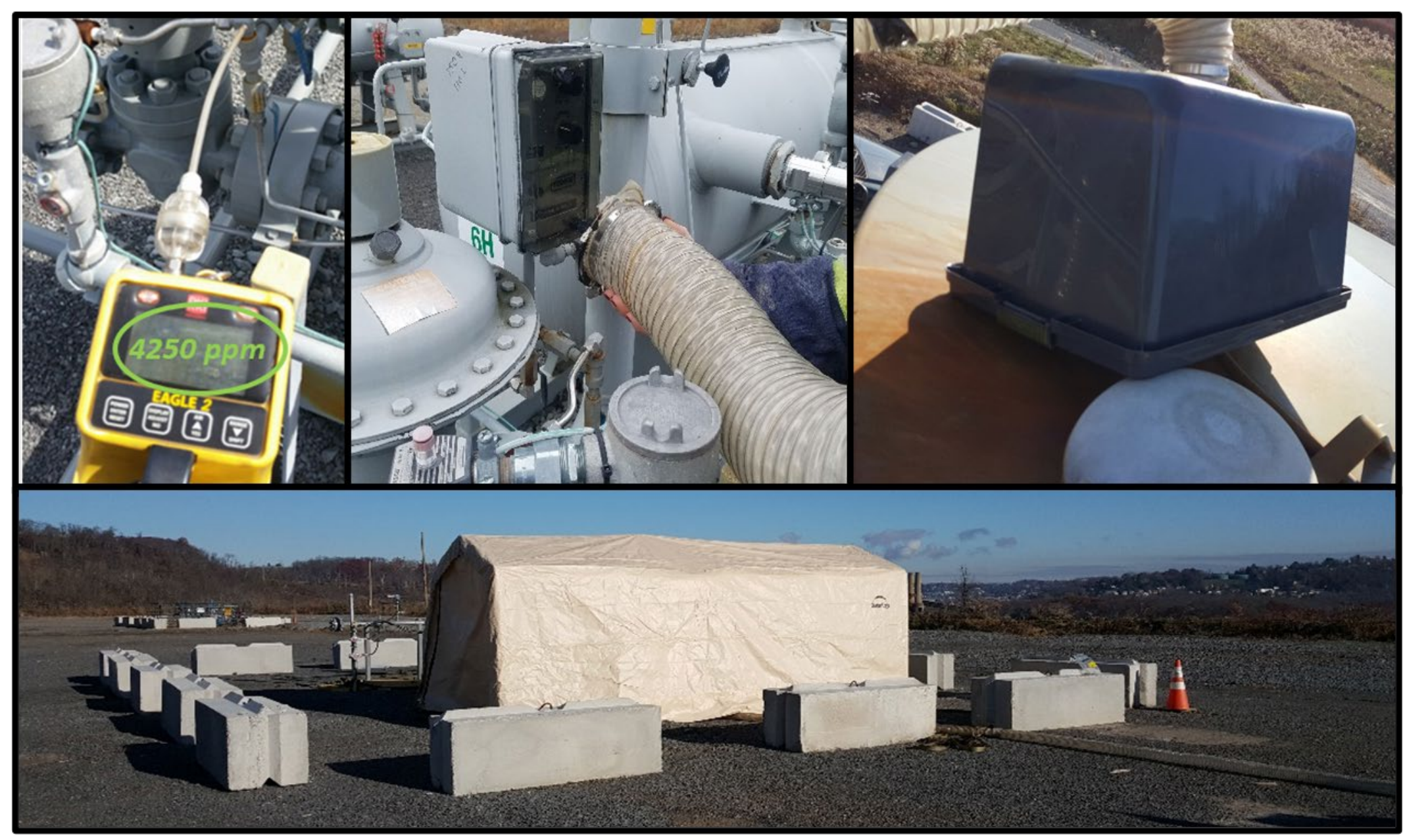

Figure 22. Detection and sampling methods used during MSEEL audits. 


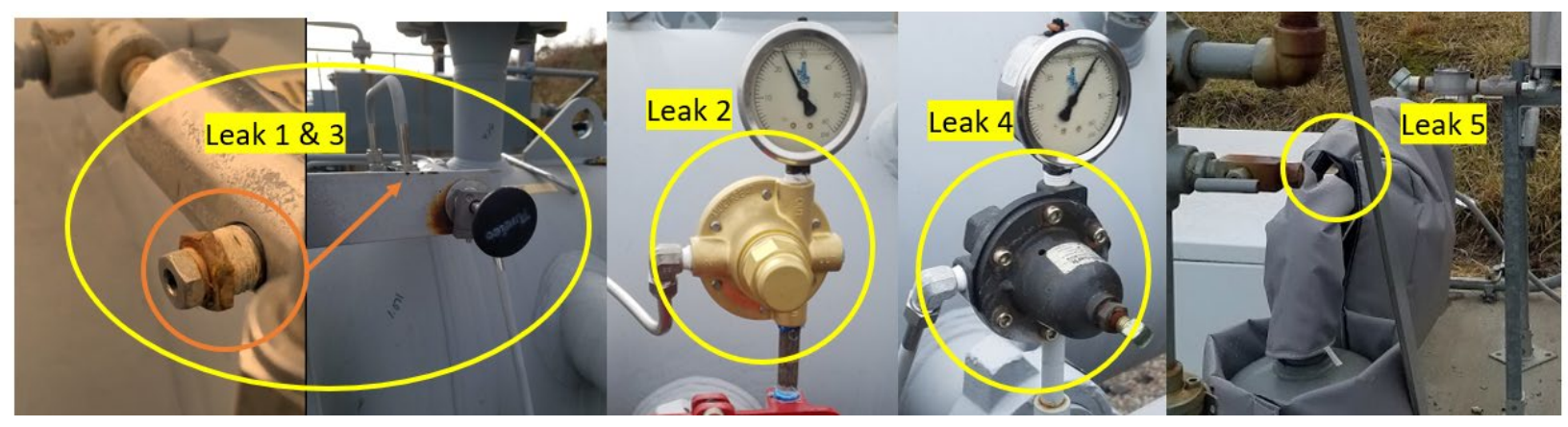

Figure 23. Examples of leaking components at MSEEL (March 2020).

There were 11 audits conducted before the deployment of the MECT. These audits also occurred before the initial controlled release experiments and were used as a point of reference during the selection of the controlled release rates. The mean and non-zero mean of the individual measurements from these audits were 0.15 and $0.17 \mathrm{~g} / \mathrm{s}$, respectively. However, the geometric mean and the median were 0.0027 and 0.0023 $\mathrm{g} / \mathrm{s}$, respectively, suggesting that most of the emissions were dominated by a few large measurements. The total site emissions displayed similar trends during the first 11 audits, as the mean emissions were $1.71 \mathrm{~g} / \mathrm{s}$, the geometric mean emissions were $0.29 \mathrm{~g} / \mathrm{s}$, and the median emissions were only $0.17 \mathrm{~g} / \mathrm{s}$. Figure 24 presents the distribution of individual and total emissions measurements from the first 11 audits. These trends are consistent with those observed by other researchers. During the first 11 audits, $90 \%$ of all measurements were less than $0.036 \mathrm{~g} / \mathrm{s}$ and $50 \%$ of measurements were a full order of magnitude less. It was acknowledged that any indirect method of quantification would not be precise enough to distinguish between individual measurements of this magnitude. To compensate for this a grouping of site components was performed based on the geometry of the site. These groups were based on the spacing of the components on the site. The four groups were Wells $4 \mathrm{H}$ and $6 \mathrm{H}$, Wells $3 \mathrm{H}$ and $5 \mathrm{H}$, the produced water tank, and the EGPUs and other associated equipment. The groups are presented in Figure 25 and their locations with respect to the MECT as it was deployed on site are presented in Table 23.

Group rate emissions were determined by combining all the measurements within the groups. It was believed that group rate emissions would be more easily quantified indirectly than individual emission rates. As such group rates were also used to get a range of possible releases for the farm data collection campaign. The mean emissions rate from the groups during the first 11 audits was $0.43 \mathrm{~g} / \mathrm{s}$, however, the geometric mean was only $0.01 \mathrm{~g} / \mathrm{s}$. The distribution of group emissions rates from these audits are presented in Figure 24. Again, the mean emission rate is dominated by a few high emitting measurements. Ninety percent of group emissions rates were less than $0.28 \mathrm{~g} / \mathrm{s}$ and $50 \%$ were less than $0.01 \mathrm{~g} / \mathrm{s}$.

All audit totals are presented in Figure 26. The blue bars represent audits that occurred before the MECT was deployed on site. Gold bars represent audits that occurred with concurrent MECT measurements. The geometric mean of total site emissions across all audits was $0.82 \mathrm{~kg} / \mathrm{hr}$, but the mean emissions were 4.2 $\mathrm{kg} / \mathrm{hr}$. This difference is a product of the fat-tailed distribution presented in Figure 24. 


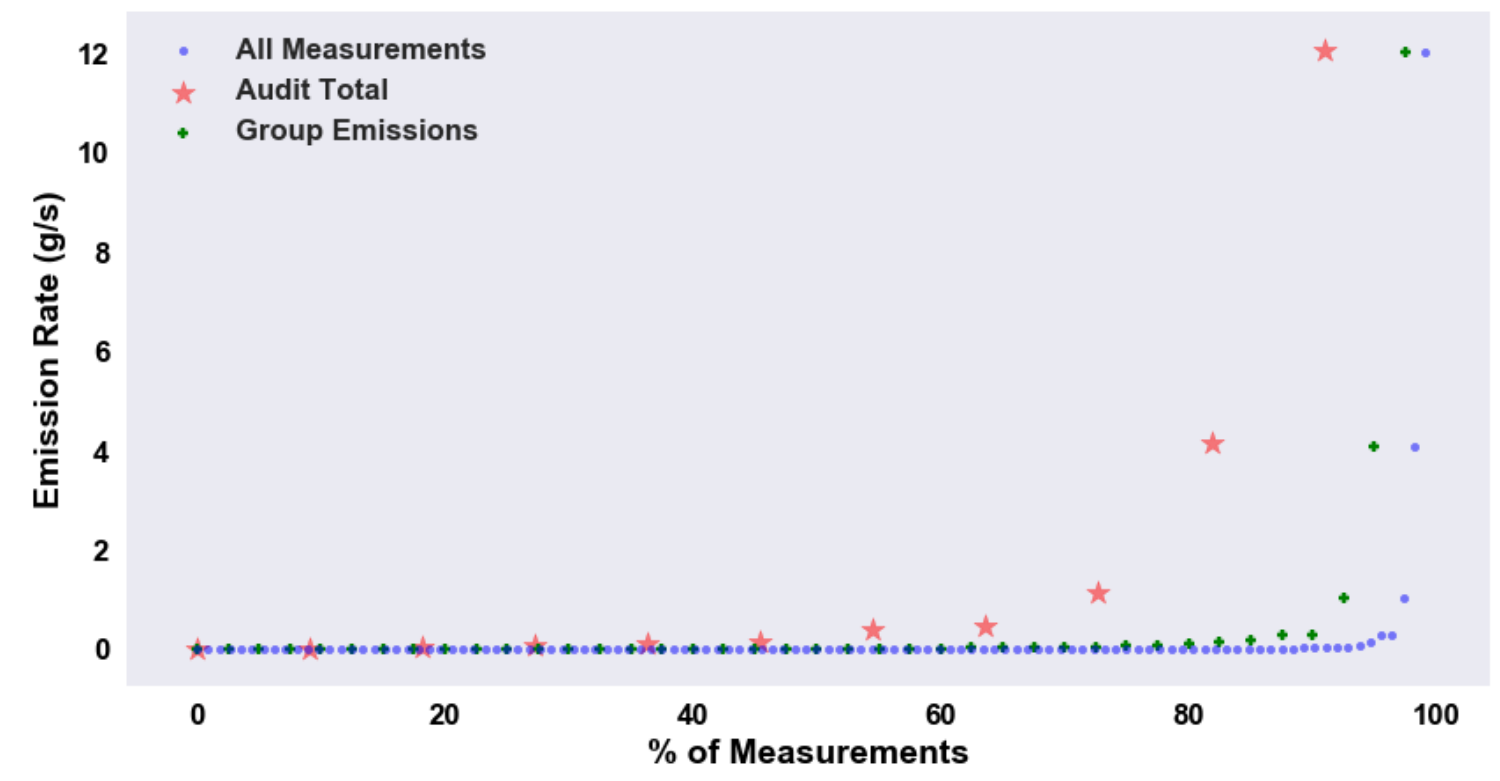

Figure 24. Distributions of methane mass emissions from the first 11 audits.

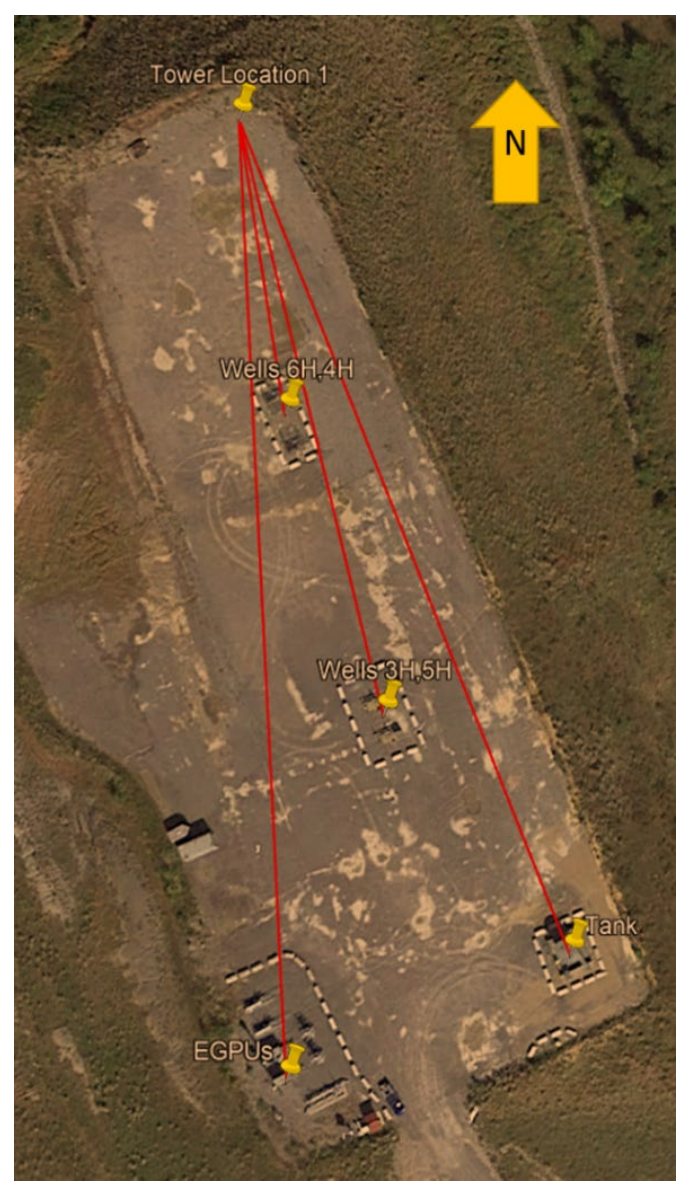

Figure 25. MECT deployment relative to component groups at MSEEL. 
Table 23. Component group bearings and distances relative to MECT location at MSEEL.

\begin{tabular}{|c|c|c|}
\hline \multirow{2}{*}{ Group } & $\begin{array}{c}\text { Bearing to } \\
\text { MECT }\end{array}$ & $\begin{array}{c}\text { Distance } \\
\text { from } \\
\text { MECT }\end{array}$ \\
\cline { 2 - 3 } & $\left({ }^{\circ}\right.$ EoN) & $(\mathbf{m})$ \\
\hline EGPU & 177 & 166 \\
\hline Well35 & 166 & 108 \\
\hline Well46 & 170 & 54 \\
\hline Tank & 157 & 159 \\
\hline
\end{tabular}


Table 24. Methane emissions from MSEEL direct quantification audits.

\begin{tabular}{|c|c|c|c|c|c|c|c|c|c|c|c|}
\hline \multicolumn{2}{|c|}{ Audit } & \multirow{2}{*}{$\begin{array}{c}\text { Background } \\
\text { Concentration } \\
\text { (ppm) }\end{array}$} & \multicolumn{4}{|c|}{ Well Emissions (g/hr) } & \multirow{2}{*}{$\begin{array}{c}\text { Tank } \\
\text { Emissions } \\
(\mathrm{g} / \mathrm{hr})\end{array}$} & \multicolumn{2}{|c|}{$\begin{array}{c}\text { EGPU } \\
\text { Emissions (g/hr) }\end{array}$} & \multirow{2}{*}{$\begin{array}{c}\text { Leak } \\
\text { Emissions } \\
(\mathrm{g} / \mathrm{hr})\end{array}$} & \multirow{2}{*}{$\begin{array}{c}\text { Total } \\
\text { Emissions } \\
(\mathrm{g} / \mathrm{hr})\end{array}$} \\
\hline \# & Date & & MIP 3H & MIP 5H & MIP 6H & MIP 4H & & $\begin{array}{c}\text { MIP } \\
5 \mathrm{H}\end{array}$ & $\begin{array}{c}\text { MIP } \\
\mathbf{3 H}\end{array}$ & & \\
\hline 1 & 2016-11 & 2.67 & 0.17 & 0.16 & 0.14 & 0.39 & 19.9 & 22.3 & 334 & 227 & 605 \\
\hline 2 & 2017-04 & 2.04 & 134 & 0 & 4.29 & 0 & 3731 & 39.5 & 30.3 & 163 & 4102 \\
\hline 3 & 2017-07 & 2.47 & 0 & 0.3 & 0.01 & 1.33 & 17.3 & N/A & 58.9 & N/A & 77.8 \\
\hline 4 & 2017-11 & 2.79 & 0.24 & 0.24 & 0.96 & 0.24 & 1032 & 0.98 & 187 & 546 & 1768 \\
\hline 5 & $2018-05$ & 2.45 & 0.32 & 0.44 & 4.66 & 0.41 & 1074 & 102 & 98 & 215 & 1496 \\
\hline 6 & 2018-08 & 2.35 & 0 & 0.16 & 0.52 & 0 & 22.4 & 38 & 32.9 & 11.8 & 106 \\
\hline 7 & 2018-10 & 2.22 & 2.1 & 0 & 38.7 & 5.88 & 43322 & N/A & N/A & 69.4 & 43438 \\
\hline 8 & 2018-12 & 3.82 & 0 & 0 & 14.6 & 14.5 & 14693 & 212 & 14.9 & 71.2 & 15020 \\
\hline 9 & 2019-02 & 2.18 & 0 & 0 & 10.7 & 4.12 & 25.5 & 110 & 0.23 & 195 & 346 \\
\hline 10 & 2019-04 & 2.2 & 0 & 0 & 18.2 & 1.52 & 26.8 & 12.7 & 0.59 & 182 & 242 \\
\hline 11 & 2019-06 & 2.15 & 0 & 0 & 199 & 1.55 & 109 & 34.9 & 0.51 & 124 & 469 \\
\hline $12 *$ & 2019-11 & 2.16 & 0.12 & $\mathbf{0}$ & 2.34 & 4.03 & 26.5 & 25.6 & 2.16 & 572 & 633 \\
\hline $13 *$ & 2020-01 & 2.25 & 0.38 & 0.09 & 2.75 & 6.05 & 21.9 & 31.5 & 1.77 & 297 & 361 \\
\hline $14 *$ & 2020-03 & 2.27 & 0.2 & 0.06 & 0.51 & 4.21 & 1467 & 7.23 & 1.2 & 928 & 2408 \\
\hline $15 *$ & 2020-06 & 2.24 & 1 & $\mathbf{0}$ & 1.34 & 5.39 & 218 & 93.4 & 0.33 & 43.6 & 363 \\
\hline $16 *$ & 2020-09 & 2.3 & 0.32 & 0.04 & 2.78 & 8.76 & 159 & 3.67 & 0.8 & 115 & 291 \\
\hline $17 *$ & 2020-11 & 2.16 & 0.38 & 0.04 & 0.49 & 1.29 & 3.47 & 31.3 & 2.28 & 393 & 432 \\
\hline
\end{tabular}


Table 25. Methane mass rate emissions statistics of all audits at MSEEL.

\begin{tabular}{|c|c|c|c|c|c|c|c|c|c|c|}
\hline \multirow{2}{*}{ Statistic } & \multirow{2}{*}{$\begin{array}{c}\text { Background } \\
\text { Concentration } \\
(\text { ppm) }\end{array}$} & \multicolumn{4}{|c|}{ Well Emissions (g/hr) } & \multirow{2}{*}{$\begin{array}{c}\text { Tank } \\
\text { Emissions } \\
(\mathrm{g} / \mathrm{hr})\end{array}$} & \multicolumn{2}{|c|}{$\begin{array}{c}\text { EGPU Emissions } \\
(\mathrm{g} / \mathrm{hr})\end{array}$} & \multirow{2}{*}{$\begin{array}{c}\text { Leak } \\
\text { Emissions } \\
(\mathrm{g} / \mathrm{hr})\end{array}$} & \multirow{2}{*}{$\begin{array}{c}\text { Total } \\
\text { Emissions } \\
(\mathrm{g} / \mathrm{hr})\end{array}$} \\
\hline & & MIP 3H & MIP 5H & MIP 6H & MIP 4H & & MIP 5H & MIP 3H & & \\
\hline Geometric Mean & 2.37 & 0.61 & 0.12 & 2.34 & 2.30 & 176 & 26.5 & 5.64 & 166 & 821 \\
\hline Mean & 2.40 & 8.17 & 0.09 & 17.8 & 3.51 & 3881 & 51.0 & 47.9 & 260 & 4245 \\
\hline Median & 2.25 & 0.20 & 0.04 & 2.75 & 1.55 & 109 & 31.5 & 2.22 & 188 & 469 \\
\hline Maximum & 3.82 & 134 & 0.44 & 199 & 14.5 & 43,322 & 212 & 334 & 928 & 43,438 \\
\hline Minimum & 2.04 & 0.00 & 0.00 & 0.01 & 0.00 & 3.47 & 0.979 & 0.234 & 11.8 & 77.8 \\
\hline Standard Deviation & 0.415 & 32.3 & 0.130 & 47.7 & 3.85 & 10,768 & 56.5 & 91.4 & 243 & 10,712 \\
\hline
\end{tabular}




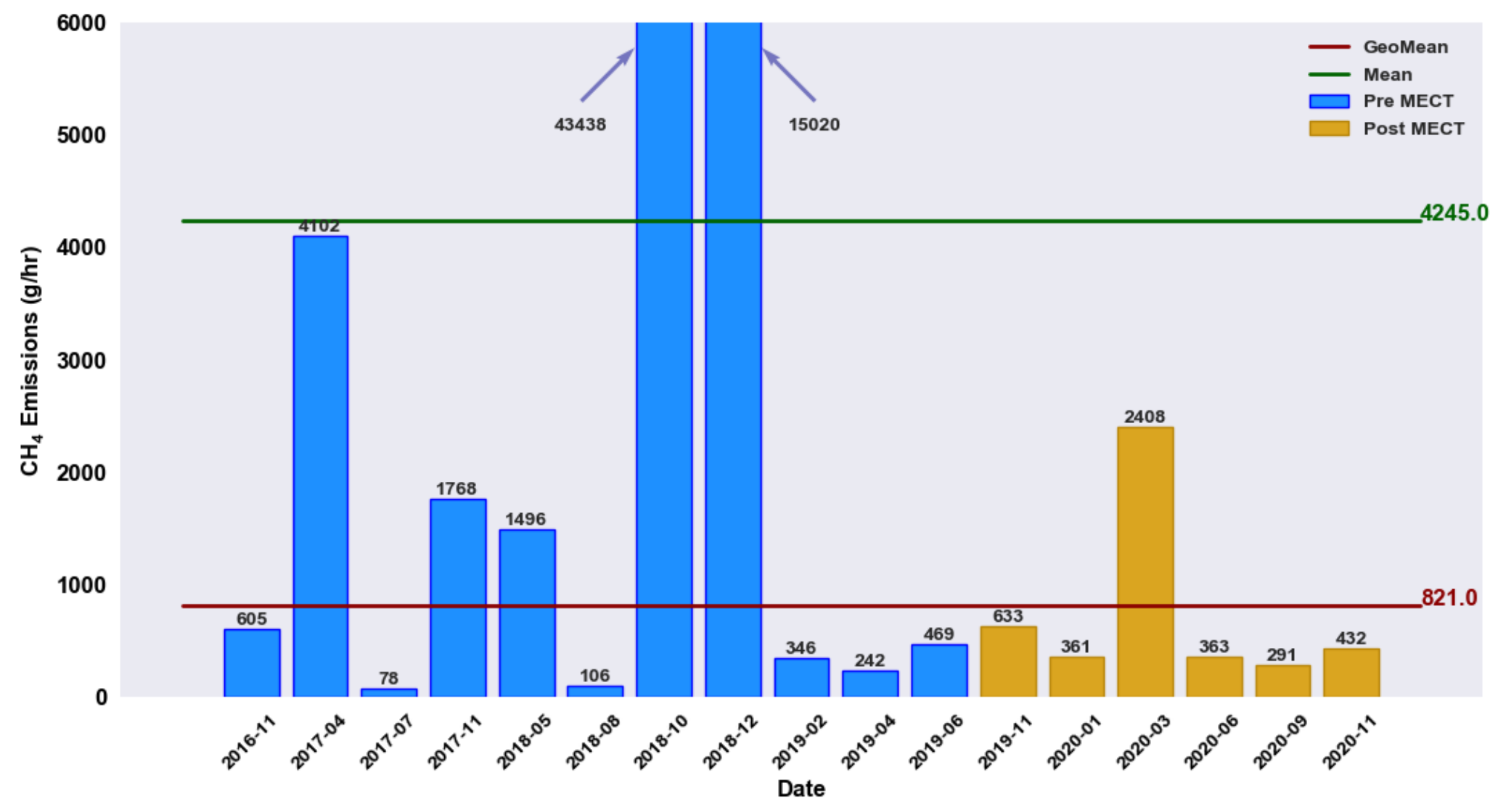

Figure 26. MSEEL audit results for total site methane emissions. 


\subsubsection{JW Ruby Research Farm Controlled Releases}

The initial deployment of the MECT occurred at the WVU JW Ruby Research Farm located in Reedsville, WV [105]. This site will subsequently be referred to as the "farm". The farm is typically utilized for forage crop production to support the Animal Sciences Farm in Morgantown but was used in this research due to its open fields available for use. The fields used contained no high canopy vegetation or building interference. Two open fields served as locations for experiments involving controlled releases of methane. These fields are normally used for cattle grazing; however, no cattle were present in the fields while they were used for experiments. It should be noted that at times the cattle were in adjacent fields and could have contributed to elevated background methane concentrations in the area. The MECT was deployed and collected data continuously during the controlled releases performed at the farm.

Knowledge of the MSEEL site was available prior to deployment of the MECT at the farm. This aided in the design of the controlled release experiments performed. The MSEEL dimensions and the results from the first 11 audits performed were accounted for when determining controlled release distances and rates. The dimension information was presented in Figure 25 and Table 23. MSEEL measurements were presented in Table 24. Controlled release rates and distances from other OTM studies were also considered as points of comparison [47], [51], [56], [60]. When designing the matrix, it was important to keep the order of magnitude of both rate and distance comparable to both MSEEL and other studies that focused on well sites and OTM measurements. The designed release/distance matrix consisted of nine different release rate/distance $(\mathrm{RRD})$ scenarios.

When setting up the controlled release experiments, the distances were approximated with a range finder. The exact distances were later calculated based on the GPS coordinates of the release point and the data collection point (the MECT location). The three distances that were targeted were $50 \mathrm{~m}, 75 \mathrm{~m}$, and $125 \mathrm{~m}$. There ended up being a total of four release distances, due to data loss during one of the controlled releases.

The average methane emission rate of all measurements made at the MSEEL site through the start of the controlled release testing was $0.12 \mathrm{~g} / \mathrm{s}$, however, the geometric mean was less than $0.003 \mathrm{~g} / \mathrm{s}$. The geometric mean was less than that of the detection limit reported by Brantley et al [50]. Budgetary and time limits were also considered when designing the release experiment matrix. A release of $100 \mathrm{~g} / \mathrm{s}$, for example, could not be maintained for sufficient time without significant costs. The final controlled release matrix consisted of rates of $0.04,0.12$, and $0.24 \mathrm{~g} / \mathrm{s}$. The release rates were controlled by the MFC at flow rates of 3,10 , and 20 standard liters per minute (slpm). The final controlled release matrix is presented in Table 26. For comparison, release rates and distances from previous controlled release experiments are presented in Table 27.

Table 26. Controlled release experiment test matrix.

\begin{tabular}{|c|c|c|c|}
\hline Release Rates (g/s) & \multicolumn{4}{|c|}{ Distances (m) } \\
\hline $\mathbf{0 . 0 3 6}$ & 42 & 72 & 119 \\
\hline $\mathbf{0 . 1 1 9}$ & 57 & 72 & 119 \\
\hline $\mathbf{0 . 2 3 9}$ & 42 & 72 & 119 \\
\hline
\end{tabular}


Table 27. Release rates and distances from previous studies.

\begin{tabular}{|c|c|c|c|c|c|}
\hline \multicolumn{2}{|c|}{ Source } & $\begin{array}{c}\text { Thoma, } \\
\text { et al }\end{array}$ & $\begin{array}{c}\text { Rella, } \\
\text { et al }\end{array}$ & $\begin{array}{c}\text { Robertson, } \\
\text { et al }\end{array}$ & $\begin{array}{c}\text { Albertson, } \\
\text { et al }\end{array}$ \\
\hline \multirow{2}{*}{$\begin{array}{c}\text { Distance } \\
(\mathbf{m})\end{array}$} & min & 18 & 5 & 34 & - \\
\cline { 2 - 6 } & mean & 70 & 34 & - & - \\
\cline { 2 - 6 } & max & 179 & 81 & 174 & - \\
\hline \multirow{2}{*}{$\begin{array}{c}\text { Release } \\
\text { Rates } \\
(\text { g/s) }\end{array}$} & min & 0.19 & 0.12 & 0.03 & - \\
\cline { 2 - 6 } & mean & 0.54 & 0.3 & - & 0.6 \\
\cline { 2 - 6 } & max & 1.2 & 0.59 & 0.56 & - \\
\hline
\end{tabular}

The controlled releases were produced from a three-bottle, pressure-controlled manifold of technical to high purity methane (98-99\% composition by volume) connected in parallel to an ALICAT MCQ-Series mass flow controller (MFC). The MFC had a range of 0 to $20 \mathrm{slpm}$ with an accuracy of $\pm 2 \%$ full-scale $(0.4$ slpm) and a repeatability of $\pm 0.2 \%$ full-scale $(0.04 \mathrm{slpm})$ [126]. So, each controlled release had a total uncertainty of $\pm 0.112 \mathrm{slpm}(0.0014 \mathrm{~g} / \mathrm{s})$. The total uncertainty was determined by Equation 15 . The MFC was stored in a ventilated trailer at the release point and was powered with a 12-volt battery. The outlet of the MFC was connected to a length of tubing with an inner diameter of 25 millimeters. The outlet of the tubing was mounted to the roof of the trailer at a height of approximately $2.3 \mathrm{~m}$. The methane was released from this point at atmospheric pressure, simulating a release from an onsite blunt body, such as a tank. The release setup is presented in Figure 27.

Combined uncertainty $_{\text {. }}$

$$
=\sqrt{\left(\frac{0.02 * \text { fullscale }}{2 \sqrt{3}}\right)^{2}+\left(\frac{0.002 * \text { fullscale }}{2 \sqrt{3}}\right)^{2}}
$$

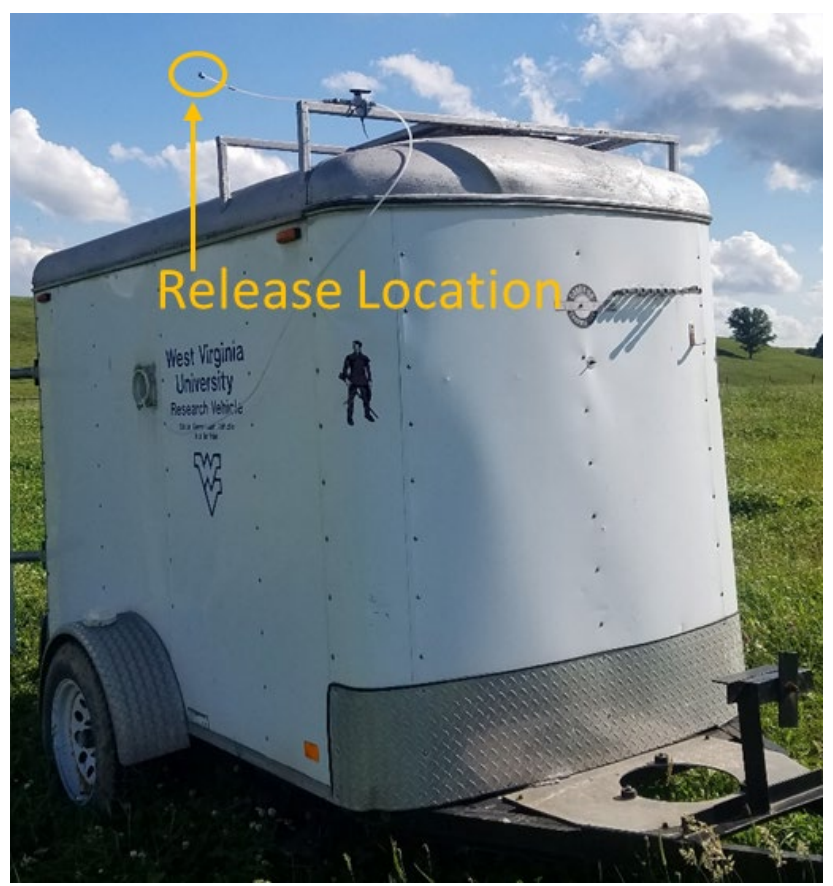

Figure 27. Controlled release trailer at the Reedsville farm. 
The controlled release trailer and MECT had to be moved several times during the data collection campaign to adjust distances or to accommodate other activities at the farm. There was a total of four separate MECT and release locations. The details of these locations are presented in Table 28 and Table 29 and on the Google Earth ${ }^{\circledR}$ image in Figure 28. The heading of the anemometer was noted for each location setup. The bearings from the controlled releases to the MECT were also calculated from the coordinates. This information was critical for filtering data based on the prevailing wind direction. Bearings and MECT heading are presented in degrees East of North $\left({ }^{\circ} \mathrm{EoN}\right)$ which was measured as the degrees clockwise from Magnetic North. For example, a bearing from the release point to the MECT of $180^{\circ} \mathrm{EoN}$ would indicate that the release was directly south of the MECT and that a southerly wind would blow from the release to the MECT.

Table 28. MECT location information at Reedsville farm.

\begin{tabular}{|c|c|c|c|c|c|c|}
\hline $\begin{array}{c}\text { MECT } \\
\text { Location } \\
\#\end{array}$ & Start Date & End Date & $\begin{array}{c}\text { Latitude } \\
\text { (DMS) }\end{array}$ & $\begin{array}{c}\text { Longitude } \\
(\mathbf{D M S})\end{array}$ & $\begin{array}{c}\text { Altitude } \\
(\mathbf{m})\end{array}$ & $\begin{array}{c}\text { Heading } \\
\left({ }^{\circ} \text { EoN) }\right.\end{array}$ \\
\hline 1 & $5 / 21 / 2019$ & $6 / 10 / 2019$ & 39.5177 & -79.8181 & 524 & 152 \\
\hline 2 & $6 / 19 / 2019$ & $7 / 11 / 2019$ & 39.5175 & -79.8173 & 522 & 0 \\
\hline 3 & $7 / 19 / 2019$ & $8 / 29 / 2019$ & 39.5192 & -79.8185 & 524 & 0 \\
\hline 4 & $8 / 29 / 2019$ & $9 / 11 / 2019$ & 39.5198 & -79.8182 & 529 & 15 \\
\hline
\end{tabular}

Table 29. Controlled release location information at Reedsville farm.

\begin{tabular}{|c|c|c|c|c|c|c|}
\hline $\begin{array}{c}\text { Release } \\
\text { Location } \\
\#\end{array}$ & Start Date & End Date & $\begin{array}{c}\text { Latitude } \\
\text { (DMS) }\end{array}$ & $\begin{array}{c}\text { Longitude } \\
\text { (DMS) }\end{array}$ & $\begin{array}{c}\text { Altitude } \\
(\mathbf{m})\end{array}$ & $\begin{array}{c}\text { Concurrent } \\
\text { MECT } \\
\text { Location \# }\end{array}$ \\
\hline 1 & $6 / 21 / 2019$ & $7 / 10 / 2019$ & 39.5175 & -79.8178 & 522 & 2 \\
\hline 2 & $7 / 31 / 2019$ & $8 / 13 / 2019$ & 39.5188 & -79.8191 & 522 & 3 \\
\hline 3 & $8 / 19 / 2019$ & $8 / 29 / 2019$ & 39.5191 & -79.8191 & 524 & 3 \\
\hline 4 & $8 / 29 / 2019$ & $9 / 11 / 2019$ & 39.5191 & -79.8192 & 524 & 4 \\
\hline
\end{tabular}




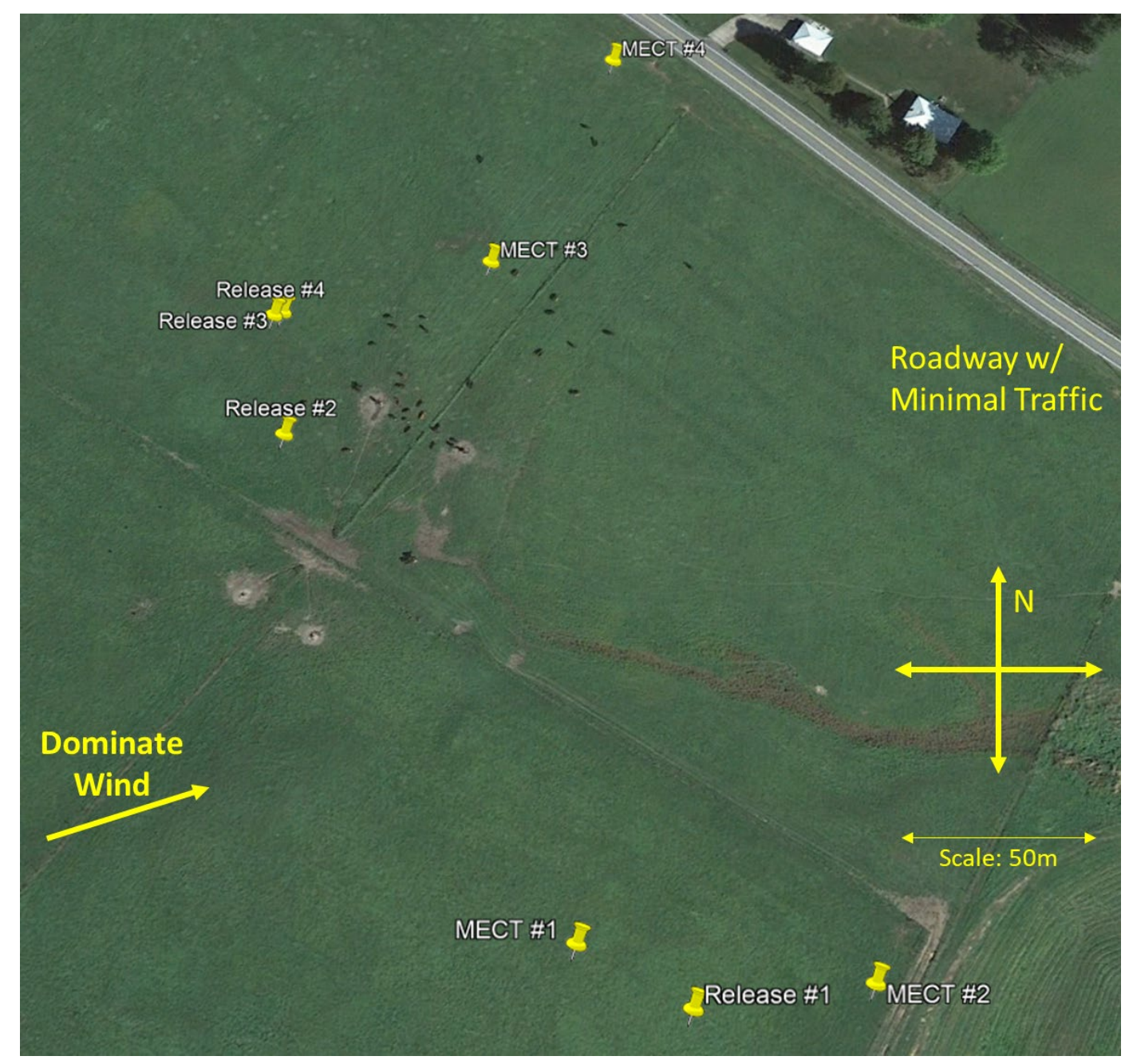

Figure 28. Map overview for controlled releases and MECT locations at Reedsville farm.

Data collection began on May 21, 2019 and concluded on September 11, 2019. Data were collected on 99 separate days during that span of time. Of the 99 days in which data were collected, 49 included some time with a controlled release. Ninety-Two of the 99 days in which data were recorded contained useful data. The seven lost days were due to a MECT DAQ failure. Data loss during the other 92 days was due to natural phenomenon that inhibited the open-path analyzer operation. This could include rain, fog, insect interference, or some other unpreventable issue and was considered "functional data loss". This type of data loss was expected to occur during any type of field data collection campaign, regardless and preparation and deployment strategy. In total, there were 2315 hours of data collected with 397 hours of functional data loss, resulting in 1918 hours of data available for analysis. The functional loss percentage was $17 \%$. Table 30 presents a breakdown of the total data collection time by release rate and distance. 
Table 30. Breakdown of controlled release data collection and loss.

\begin{tabular}{|c|c|c|c|c|c|c|c|c|}
\hline \multirow{2}{*}{ Release ID } & $\begin{array}{c}\text { Release } \\
\text { Rate }\end{array}$ & Distance & Bearing & Total & \multicolumn{2}{|c|}{ LI-7700 Data } & \multicolumn{2}{|c|}{ LI-7500DS Data } \\
\cline { 2 - 8 } & $\mathbf{( g / s )}$ & $\mathbf{( m )}$ & $\mathbf{(}{ }^{\mathbf{C} \text { EoN) }}$ & $\mathbf{( h r s )}$ & Loss (hrs) & $\begin{array}{c}\text { Valid } \\
\text { (hrs) }\end{array}$ & $\begin{array}{c}\text { Loss } \\
\text { (hrs) }\end{array}$ & $\begin{array}{c}\text { Valid } \\
\text { (hrs) }\end{array}$ \\
\hline $\begin{array}{c}\text { Initial } \\
\text { Background }\end{array}$ & 0.00 & N/A & N/A & 482 & 31 & 451 & 29 & 453 \\
\hline Initial Leak & 0.04 & 42 & 262 & 33 & 0 & 33 & 3 & 30 \\
\hline 03_050_0 & 0.04 & 42 & 262 & 123 & 20 & 103 & 32 & 91 \\
\hline 10_050_0 & 0.12 & 42 & 262 & 76 & 14 & 62 & 76 & 0 \\
\hline 20_050_0 & 0.24 & 42 & 262 & 14 & 0 & 14 & 0 & 14 \\
\hline 20_050_1 & 0.24 & 42 & 262 & 9 & 0 & 9 & 0 & 9 \\
\hline 03_075_0 & 0.04 & 72 & 225 & 122 & 40 & 82 & 1 & 121 \\
\hline 10_075_0 & 0.12 & 72 & 225 & 40 & 0 & 40 & 0 & 40 \\
\hline 20_075_0 & 0.24 & 72 & 225 & 14 & 0 & 14 & 0 & 14 \\
\hline 10_050_1 & 0.12 & 57 & 254 & 49 & 16 & 33 & 1 & 48 \\
\hline 03_100_0 & 0.04 & 119 & 228 & 114 & 27 & 88 & 5 & 109 \\
\hline 10_100_0 & 0.12 & 119 & 228 & 24 & 0 & 24 & 1 & 24 \\
\hline 10_100_1 & 0.12 & 119 & 228 & 32 & 1 & 30 & 1 & 31 \\
\hline 20_100_0 & 0.24 & 119 & 228 & 7 & 0 & 7 & 0 & 7 \\
\hline
\end{tabular}

\subsection{Data Processing}

Two primary methods of conventional data processing were used, OTM and EC. These methods employ different reasoning to quantify fluxes as previously discussed. OTM primarily focuses on measuring a horizontal flux based on atmospheric dispersion modeling. EC utilizes covariances between vertical wind components and methane to estimate a vertical gas flux. The same data periods were analyzed with both methods so that they could be evaluated simultaneously. This allowed for each methods' results to be used in a combined method to estimate emissions rates from a given period.

\subsubsection{Other Test Method 33A}

The first "conventional" method of data processing used was OTM. As previously stated, OTM was developed as an indirect quantification method for methane emissions from NG sites and relies on the principles of Gaussian dispersion. However, to understand better the nature of the calculations they were investigated in detail. The EPA has published the source code for their OTM calculations, which was used as a template for the code used in data processing [49].

It was critical for the success of the method that the emissions concentration and wind data were time aligned. The EPA applied sampling delay corrections since the emissions analyzer used relied on an internal pump. The CMI used in this research was open-path and recorded measurements at the same frequency as the sonic anemometer and therefore time delay was deemed negligible.

OTM requires five primary inputs, in addition to raw data, that govern the calculations of the method. These values will be referred to as "binning parameters" because they influence the binning of data by either concentration, wind direction or volume of data. The default OTM binning parameters are presented in Table 31 . 
Table 31. OTM binning parameters and their default values.

\begin{tabular}{|l|c|}
\hline Parameter & Value \\
\hline Wind Direction Bin Size $\left(^{\circ}\right)$ & 10 \\
\hline Wind Speed Limit $(\mathrm{m} / \mathrm{s})$ & 0 \\
\hline Bin Angle Limit $\left({ }^{\circ}\right)$ & 60 \\
\hline Bin Density Limit $(\%)$ & 2 \\
\hline Background Limit $(\%)$ & 5 \\
\hline
\end{tabular}

The first step in processing was to "streamline" the wind speed measurements. This consisted of rotating the axis of wind measurement so that the mean variations along the $\mathrm{x}$ and $\mathrm{z}$ axes were zero. The streamline correction essentially acted to determine the horizontal wind speed and direction. Initial binning was then applied to the methane concentration data. For data binning the following steps were taken:

1. The calculated wind direction was segmented into bins based on the Wind Direction Bin Size $\left(\right.$ default $\left.=10^{\circ}\right)$.

2. The corresponding methane concentrations were placed into bins based the wind direction recorded at the same instant.

3. The methane concentrations of each bin were averaged. Any bin that contained no methane data was assigned a mean value of zero.

4. The values of average methane concentration were rotated so that the peak concentration was near the $180^{\circ}$ point on a $0-360^{\circ}$ axis.

After data binning and alignment two steps were taken to eliminate data. The first was the "Wind Speed Limit" cut. During this step, any data point with a horizontal wind speed less than the cut off value was eliminated, though for the default processing the value was defined as zero, so no data were eliminated. The second was the "Bin Angle Limit" cut. This involved eliminating all data that were in a bin outside of the "Bin Angle Limit" (default $=60^{\circ}$ ). This meant that after data were binned only those within $\pm 60^{\circ}$ of the peak average methane concentration were retained. The methane background level was then determined. The background concentration was determined as the mean of the lowest "Background Limit" percent (default $=5 \%$ ) of methane concentrations from the period. This value was then subtracted from each remaining bin averaged methane value to produce the average elevated methane level of each bin. The data was then re-binned as before. The "Bin Density Limit" cut (default $=2 \%$ ) was then applied to the bins. During this step any bin that contained less than the "Bin Density Limit" percentage of the total data in the period was eliminated. The data were then re-centered to ensure that the peak was as near to the $180^{\circ}$ as possible (depending on the bin size). These final binned values of average methane were fitted using a Gaussian curve. The Gaussian equation to which the data were fit is presented in Equation 16.

$$
f(x)=a * e^{\left(\frac{-(x-b)^{2}}{2 c^{2}}\right)}
$$

Equation 16

Where,

- $\quad a$ is the average methane concentration of the highest bin

- $\quad b$ is the center angle $\left(\sim 180^{\circ}\right)$

- $\quad c$ is the width of the fit $\left(10-120^{\circ}\right)$

After all curve fitting the statistics of the remaining data were calculated. This produced values that were necessary for determining the stability class, as well as making temperature and pressure corrections. The 
mean pressure, temperature, and horizontal wind speed and the standard deviations of vertical wind speed and wind direction were all required statistics. The turbulence intensity (TI) was calculated by dividing the standard deviation of the vertical wind speed by the mean of the horizontal wind speed. The ASI was then defined based on the TI and the standard deviation of the horizontal wind direction. The ASI was based on the traditionally used Pasquill stability classes and ranges from 1-7. The ASI was determined by taking an average of values of the stability definitions based on the two variables used. Table 32 presents the ranges of each variable and their corresponding contribution to the ASI.

Table 32. OTM ASI values.

\begin{tabular}{|c|c|c|}
\hline $\begin{array}{c}\text { Standard Deviation of Horizontal } \\
\text { Wind Speed (ws) }\end{array}$ & Turbulence Intensity (TI) & $\begin{array}{c}\text { ASI } \\
\text { Contribution }\end{array}$ \\
\hline $27.5<$ ws & $0.205<\mathrm{TI}$ & 1 \\
\hline $23.5<\mathrm{ws}<=27.5$ & $0.180<\mathrm{TI}<=0.205$ & 2 \\
\hline $19.5<\mathrm{ws}<=23.5$ & $0.155<\mathrm{TI}<=0.180$ & 3 \\
\hline $15.5<\mathrm{ws}<=19.5$ & $0.130<\mathrm{TI}<=0.155$ & 4 \\
\hline $11.5<\mathrm{ws}<=15.5$ & $0.105<\mathrm{TI}<=0.130$ & 5 \\
\hline $7.5<\mathrm{ws}<=11.5$ & $0.080<\mathrm{TI}<=0.105$ & 6 \\
\hline ws $<=7.5$ & $\mathrm{TI}<=0.080$ & 7 \\
\hline
\end{tabular}

Once the average ASI was determined, it was used along with the estimated distance from the source to the measurement location as the column and index for look-up tables to determine the $\mathrm{y}$ and $\mathrm{z}$ stability coefficients. The density of the dispersed methane was then calculated by using the maximum of the methane Gaussian curve fit and correcting using the mean pressure and temperature as defined by Equation 17 . This value was then multiplied by $2 \pi$, the stability coefficients, and the mean wind speed to produce the final mass emission estimate which is presented in Equation 18.

$$
\begin{aligned}
& \mathrm{CH}_{4} \text { Density }\left(\frac{g}{m^{3}}\right) \\
& =\text { Peak Fit } \mathrm{CH}_{4}(\mathrm{ppm}) * \frac{16.04 \mathrm{~g}_{\mathrm{CH}_{4}}}{1 \mathrm{~mol}_{\mathrm{CH}_{4}}} *\left(\frac{1000 \mathrm{~L}}{1 \mathrm{~m}^{3}}\right) \\
& \text { * } 1 e^{-6} \\
& *\left(\frac{1 \mathrm{~mol} * K}{8.314 L * k P a}\right)\left(\frac{\text { MeanPressure }(k P a)}{\text { MeanTemperature }(K)}\right) \\
& \mathrm{CH}_{4} \text { MassRate }\left(\frac{g}{s}\right) \\
& =C H_{4} \text { Density }\left(\frac{g}{m^{3}}\right) * 2 \pi * \sigma_{y}(m) * \sigma_{z}(m) \\
& \text { *Wind Speed mean horizontal }\left(\frac{m}{s}\right)
\end{aligned}
$$

All OTM results from this research were calculated using Python 3.6 [127] scripts that were based on the OTM codes released by the EPA, which were developed in MATLAB $^{\circledR}$ R2015a [128]. These codes are available online along with test datasets and results obtained from those datasets [49]. The data provided 
by the EPA were recorded at $10 \mathrm{~Hz}$, which was the same data rate used in this research. This provided a method of verification to ensure that the Python scripts were correctly calculating results. The Python results were compared to the EPA results from code "120415_Histcount.m" for 20 different raw data files. All the data files were recorded during tests where the known release rate was $0.6 \mathrm{~g} / \mathrm{s}$. The results of the two calculations are presented in Table 33.

Table 33. Results from comparison between EPA MATLAB ${ }^{\circledR}$ and Python analyses.

\begin{tabular}{|l|c|c|c|c|}
\hline $\begin{array}{c}\text { EPA Release Excel } \\
\text { Filename }\end{array}$ & $\begin{array}{c}\text { Distance } \\
(\mathbf{m})\end{array}$ & $\begin{array}{c}\text { EPA } \\
\text { MATLAB } \mathbb{8} \\
\text { Results (g/s) }\end{array}$ & $\begin{array}{c}\text { Python } \\
\text { Results } \\
\mathbf{( g / s )}\end{array}$ & Difference \\
\hline STR_4042011_01.xls & 40 & 0.43 & 0.43 & $0.0 \%$ \\
\hline STR_4042011_03.xls & 60 & 0.54 & 0.77 & $\mathbf{4 2 . 7 \%}$ \\
\hline STR_4042011_05.xls & 35 & 0.75 & 0.75 & $0.0 \%$ \\
\hline STR_4042011_06.xls & 97 & 0.38 & 0.38 & $0.0 \%$ \\
\hline STR_4042011_07.xls & 57 & 0.53 & 0.53 & $0.0 \%$ \\
\hline STR_5050611_01.xls & 88 & 0.64 & 0.65 & $0.0 \%$ \\
\hline STR_5050611_02.xls & 88 & 0.61 & 0.61 & $0.0 \%$ \\
\hline STR_5050611_03.xls & 98 & 0.38 & 0.37 & $\mathbf{3 . 2 \%}$ \\
\hline STR_5050611_04.xls & 98 & 0.74 & 0.74 & $0.0 \%$ \\
\hline STR_5050611_05.xls & 103 & 0.64 & 0.64 & $0.0 \%$ \\
\hline STR_5050611_06.xls & 103 & 0.31 & 0.31 & $0.0 \%$ \\
\hline STR_5050611_07.xls & 103 & 0.35 & 0.35 & $0.0 \%$ \\
\hline STR_5050611_08.xls & 82 & 0.61 & 0.61 & $0.0 \%$ \\
\hline STR_5050611_09.xls & 57 & 0.80 & 0.75 & $\mathbf{5 . 7 \%}$ \\
\hline STR_5050611_10.xls & 57 & 0.63 & 0.63 & $0.0 \%$ \\
\hline STR_6061411_01.xls & 81 & 0.59 & 0.59 & $0.0 \%$ \\
\hline STR_606141103.xls & 98 & 0.32 & 0.32 & $0.0 \%$ \\
\hline STR_6061411_04.xls & 65 & 0.51 & 0.76 & $47.8 \%$ \\
\hline STR_6061411_05.xls & 41 & 0.49 & 0.49 & $0.0 \%$ \\
\hline STR_6061411_06.xls & 93 & 0.60 & 0.60 & $0.0 \%$ \\
\hline
\end{tabular}

Table 33 highlights the primary difference in the two methods of calculation. The differences in the calculations stem from a different wind speed used for stability class calculations. The OTM method relied on a look-up table to determine stability class. These look-up tables are published by the EPA along with the OTM method results [49]. The stability class was governed by three variables: distance from the source, the standard deviation of the wind direction, and the TI. The wind speed components differ in the two methods of calculations due to the wind measurements available. The Python script was the same one used for data processing of the data collected under this research. It utilized local 3-D sonic anemometer wind speed data to calculate these values, while in the MATLAB ${ }^{\circledR}$ code a nearby micrometeorological station's 2-D wind data were used. The reason for this difference was based on the research being conducted. The MECT was meant to be a standalone system that did not require other sources of data for indirect quantification. Therefore, only those data that were recorded by the station were to be used for the application of this research. There is no guarantee that local micrometeorological data would be available at the time and place of deployment. Even with these differences in calculation methods, the same results were obtained $75 \%$ of the time (16 of 20 cases). Of the four files that produced different results, two 
produced a difference less than $6 \%$ and two produced a difference between $40 \%$ and $50 \%$. This analysis highlighted the potential sensitivity of such methods to arbitrarily defined values such as atmospheric stability and emphasizes the stepwise nature of stability class definition. There is no smooth transition between classes and the coefficients determined by the values can change drastically based on only small differences in the raw data.

\subsubsection{Eddy Covariance}

The second of the two "conventional" data processing methods was EC, the theory of which was described in Section 2.2.3.2. There are many specific details that go into EC processing. Several authors have developed a variety of EC processing techniques, some of which have been widely adopted by the EC community. Several programs have been developed to process raw EC data and produce fluxes. A few examples of those that are widely accepted by the EC community include: EddySoft, EddyPro, TK3, EddyUH, EdieRe, eddy4R-Docker, and EC-Frame [129]. One of the most prominent in recent years has been EddyPro ${ }^{\circledR}$, which was developed by LI-COR, Inc. EddyPro ${ }^{\circledR}$ is based on the free, open-source $\mathrm{ECO}_{2} \mathrm{~S}$ package which was developed by the Infrastructure for Measurement of the European Carbon Cycle (IMECC-EU) [130]. The development of the first version of EddyPro ${ }^{\circledR}$ began in 2010 and in 2019 LI-COR released the 7.0 version which was the most up to date version available when this data analysis began. EddyPro ${ }^{\circledR}$ was used in this research due to its user-friendly interface and easy integration with LI-COR devices such as the LI-7700 and LI-7500DS, which were both used in this research. EddyPro ${ }^{\circledR}$ offers an "Express Mode" which allows the user to quickly calculated EC fluxes using pre-determined processing settings which are widely accepted by the research community. The software also offers an "Advanced Mode" in which processing settings can be adjusted based on the specific data collection setup and processing goals. As a point of comparison, data were processed first using the "Express Settings". It was established that systematic and processing errors are a large source of uncertainty in EC measurements. The default EddyPro ${ }^{\circledR}$ general processing settings are presented in Table 34, the statistical analysis settings are presented in Table 35, and spectral analysis settings are presented in Table 36 [131].

Table 34. EddyPro ${ }^{\circledR}$ "Express" settings [131].

\begin{tabular}{|l|c|}
\hline Basic Settings & $10 \%$ \\
\hline Missing samples allowance & 15 min \\
\hline Flux averaging interval & None \\
\hline Flags RSSI & \multicolumn{1}{|c|}{} \\
\hline Advanced Settings & Selection \\
\hline Processing Options & Yes \\
\hline Fix 'w' boost & Double Rotation \\
\hline Angle of attack correction for wind components & Block Average \\
\hline Axis for Rotations & Covariance maximization with default \\
\hline Turbulent Fluctuations & Yes \\
\hline Time lags compensation & Kljun et al (2004) \\
\hline Compensate density fluctuations (WPL) & \\
\hline Quality Check & Mauder and Foken (2004) 0-1-2 system \\
\hline Footprint Estimation & K \\
\hline
\end{tabular}


Table 35. EddyPro ${ }^{\circledR}$ “Express” statistical analysis settings [131].

\begin{tabular}{|c|c|c|c|}
\hline Statistical Analysis & \multicolumn{3}{|c|}{ Selection } \\
\hline Spike count/removal & \multicolumn{3}{|c|}{ Vickers and Mahrt (1997) } \\
\hline Amplitude resolution & \multicolumn{3}{|c|}{$7 \sigma, 100$ bins, $70 \%$ acceptance } \\
\hline Drop-outs & \multicolumn{3}{|c|}{10 percentile, $10 \%$ central, $6 \%$ extreme } \\
\hline \multirow{7}{*}{ Absolute limits } & Variable & Lower & Upper \\
\hline & $\mathrm{U}(\mathrm{m} / \mathrm{s})$ & -30 & 30 \\
\hline & $\mathrm{W}(\mathrm{m} / \mathrm{s})$ & -5 & 5 \\
\hline & $\mathrm{T}\left({ }^{\circ} \mathrm{C}\right)$ & -40 & 50 \\
\hline & $\mathrm{CO}_{2}(\mu \mathrm{mol} / \mathrm{mol})$ & 200 & 900 \\
\hline & $\mathrm{H}_{2} \mathrm{O}(\mu \mathrm{mol} / \mathrm{mol})$ & 0 & 40 \\
\hline & $\mathrm{CH}_{4}(\mu \mathrm{mol} / \mathrm{mol})$ & 0.17 & 1000 \\
\hline \multirow{5}{*}{ Skewness \& kurtosis } & Parameter & Hard Flag & Soft Flag \\
\hline & Skewness Lower & -2 & -1 \\
\hline & Skewness Upper & 2 & 1 \\
\hline & Kurtosis Lower & 1 & 2 \\
\hline & Kurtosis Upper & 8 & 5 \\
\hline Discontinuities & \multicolumn{3}{|c|}{ No } \\
\hline Time lags & \multicolumn{3}{|c|}{ No } \\
\hline Angle of Attack & \multicolumn{3}{|c|}{ No } \\
\hline Steadiness of Horizontal Wind & \multicolumn{3}{|c|}{ No } \\
\hline Random Uncertainty & \multicolumn{3}{|c|}{ No } \\
\hline
\end{tabular}

Table 36. EddyPro ${ }^{\circledR}$ “Express” spectral analysis settings [131].

\begin{tabular}{|l|c|}
\hline Spectral Analysis and Corrections & Selection \\
\hline Binned (co)spectra files not available & Yes \\
\hline Tapering Window & Hamming \\
\hline Frequency Bins for (co)spectra reduction & 50 \\
\hline Use power-of-two samples to speed up FFT & Yes \\
\hline Lowest Noise Frequency & $\mathrm{CO}_{2}: 1 \mathrm{~Hz}, \mathrm{H}_{2} \mathrm{O}: 1 \mathrm{~Hz}, \mathrm{CH}_{4}: 1 \mathrm{~Hz}$ \\
\hline $\begin{array}{l}\text { Minimum number of (co)spectra for valid } \\
\text { averages }\end{array}$ & 10 \\
\hline Low frequency range & Moncrieff et al (2004) \\
\hline High frequency range & Moncrieff et al (1997) - fully analytic \\
\hline
\end{tabular}

The extensive details of EC processing were beyond the scope of this research, however, LICOR® provides reasoning for these settings and the use of most of them is well documented in literature [66]-[68], [71], [72]. Vickers and Mahrt (1997) is widely accepted as the gold standard for data filtering and was used in 
most data processing methods as was Moncrief et al (2004) for frequency detection and correction. WPL terms have been nearly universally applied to EC processing methods [132]-[134]. Mauder and Foken (2004) has been widely used for quality control of flux measurements. For this research it was also utilized. The system relies on a rating system of $0-1-2$, which have the following meanings:

0. Fluxes of the highest quality (can always be used).

1. Fluxes suitable for general analysis such as annual budgets.

2. Fluxes that should be discarded from the results dataset [135].

Based on the setup of the system (instrumentation, placement, goals) optimal settings in EddyPro® were used to attempt to minimize the systematic errors associated with the EC calculations. The settings selected based on previous literature and suggestions from more experienced users of the EC method. The settings that were changed for the optimal settings are presented in Table 37.

Table 37. Updated EddyPro ${ }^{\circledR}$ processing settings.

\begin{tabular}{|l|c|}
\hline \multicolumn{2}{|c|}{ Advanced Settings } \\
\hline Processing Options & Details \\
\hline Angle of attack correction for wind components & Field Calibration (Nakai and Shimoyama, 2012) \\
\hline Axis for Rotations & Triple rotation \\
\hline Statistical Analysis & Details \\
\hline Time lags & $\begin{array}{c}\text { Accepted Covariance Difference (hard-flag): } 20 \%, \\
\text { (soft-flag): } 10 \%, \text { Nominal time lag: } 0 \mathrm{~s}\end{array}$ \\
\hline Angle of Attack & $\begin{array}{c}\text { Minimum: }-30^{\circ}, \text { Maximum: } 30^{\circ}, \text { Accepted Number of } \\
\text { Outliers: } 10 \%\end{array}$ \\
\hline Random Uncertainty & F\&S \\
\hline Definition of the Integral Turbulence Scale (ITS) & Cross-correlation first crossing $1 / \mathrm{e}$ \\
\hline Maximum correlation period & $10 \mathrm{~s}$ \\
\hline
\end{tabular}

Most of these were related to wind measurement as these settings can change based on the anemometer utilized. The angle of attack correction was applied using the method of Nakai and Shimoyama (2012) [136]. This correction compensates for tilt in the anemometer which was considered likely to occur with a small tower subject to movement. By the same logic, the axis for rotation setting was also switched from double rotation to triple rotation. Other than these two changes based on the anemometer, the only other major adjustment was the addition of a random uncertainty estimation based on the method of F\&S [83]. This adjustment did not affect flux calculations but added the uncertainty estimate to the EddyProß output.

\section{Data Analysis}

The analysis performed in this research stemmed from the two primary methods of data processing, OTM and EC. Much of the analysis was based on the controlled release experiments of the Reedsville Farm. The data collection approach to this campaign was described in 3.1.4. From these collected data OTM and EC results were obtained. The advantage of using this data for initial analysis was that it was well understood in terms of mass emissions rates and relative positions between the controlled releases and MECT.

To combine the results of the two methods effectively, it was desirable for both methods to have the same averaging period. EC results are typically processed using a time scale of 30 minutes [131] while OTM initially recommended data collection times between 15 and 20 minutes [47]. The goal of this research was to combine both methods so that they could be used to improve predicted emissions rates. Ultimately the averaging period selected was 15 minutes for several reasons. 
1. Increase size of the dataset. Using a period of 15 minutes rather than 30 nearly doubled the amount of usable data.

2. More refined temporal information. Methane emissions are known to be highly temporal, even on a smaller time scale. Pneumatics and other intermittently functioning devices might not be consistent across longer periods.

3. Other studies using EC to quantify point source emissions found that shorter averaging periods (15minutes) resulted in more accurate mass emissions estimation [67], [68].

Analysis of the controlled releases began with determining the proportion of the data that could be used for analysis. Collected data were only valuable for assessing mass emissions rates of controlled releases if the releases could be measured. The nature of utilizing point measurements in a stochastic environment renders a large amount of data unusable. Any point source measurement, whether EC or OTM, which relies on micrometeorological conditions is inherently difficult or impossible to apply to any situation. Due to the stochastic nature of wind, much of the data collected occurred when the wind was blowing from some direction other than that of the source. This is the primary reason that OTM was designed as a mobile platform and the reason that EC experimental design and tower placement are such critical steps to obtaining accurate, usable data [47], [64]. Without a prevailing wind in the StS direction, no useful information about the source would be obtained. Therefore, the first step to determine the amount of useful data obtained was to understand the wind direction with respect to the site setup as a function of time.

Default processed OTM and EC results were used to determine valid periods. From each of the 15-minute averaging periods an average wind direction was obtained. Since the StS wind direction was known during the controlled releases, the amount of usable data could be obtained from this method. Averaging periods used for analysis were only those that produced a valid output of the sought-after estimate (OTM: valid release rate estimation, EC: valid methane flux estimation) for both methods. Averaging periods were considered within the wind filter if the mean wind direction was within $\pm 45^{\circ}$ of the StS direction and will be referred to as wind filtered (WF). The $\pm 45^{\circ}$ designation was selected based on several EC footprint studies which used filters between $\pm 40-45^{\circ}$ [67], [68], [80].

During the entire Reedsville farm deployment there were a possible 10,878 15-minute periods. Of those, $7283(67 \%)$ resulted in valid OTM and EddyPro ${ }^{\circledR}$ calculations. Of those 7283 valid OTM estimations, $4599(63 \%)$ were from periods without a release present and $2684(37 \%)$ were from periods with a release. Of the 2684 valid periods during controlled release periods, $804(30 \%)$ were within $\pm 45^{\circ}$ of the StS direction. Of the 4459 valid background periods, 3124 (68\%) were from after the first controlled release was initiated. Of these 3124 periods, $1208(39 \%)$ of them were within $\pm 45^{\circ}$ of the StS direction. These were considered the final background periods and were used for much of the analysis. The final available dataset consisted of 15-minute periods recorded after June 20, 2019 (the day the first release was initiated), that produced valid default OTM estimates and EddyPro ${ }^{\circledR}$ methane flux estimates and had a wind direction within $\pm 45^{\circ}$ of the StS direction. The final number of background periods with these properties was 1208 and the final number of controlled release periods with these properties was 804 . The date and time indices of these periods were saved and used for all subsequent evaluations. The valid periods broken down by distance and release rate are presented in Table 38. A complete breakdown of the periods is presented in Table 39. A statistical breakdown of key variables from the 804 valid controlled release periods is presented in Table 40. Wind speed was considered one of the most critical variables for the success of both methods. The average 15 -minute period mean wind speed was $1.6 \mathrm{~m} / \mathrm{s}$ and the maximum was $5.3 \mathrm{~m} / \mathrm{s}$. The maximum observed wind speed in this dataset was $14 \mathrm{~m} / \mathrm{s}$. This aligns with the recommendation of Robertson et al. that OTM measurements were most effective with average wind speeds of 1 to $10 \mathrm{~m} / \mathrm{s}$. The controlled releases of Robertson et al. experienced ASI values between 2-6 [51]. Edie et al. performed controlled release experiments with wind speeds between 2 and $8 \mathrm{~m} / \mathrm{s}$ and ASIs between 3-6 [53]. The ASI values of this work varied between 2-7 but tended to be higher with a mean and median value of 5, which suggested that conditions tended to be more stable. 
Table 38. Number of valid controlled release periods.

\begin{tabular}{|c|c|c|c|c|c|}
\hline \multirow{2}{*}{$\begin{array}{c}\text { Distance } \\
(\mathbf{m})\end{array}$} & None & $\mathbf{0 . 0 3 6}$ & $\mathbf{0 . 1 1 9}$ & $\mathbf{0 . 2 3 9}$ & Total \\
\cline { 2 - 6 } & 577 & 234 & 110 & 38 & 382 \\
\hline 42 & 224 & - & 47 & 34 & 81 \\
\hline 57 & 289 & 63 & 100 & - & 163 \\
\hline 72 & 118 & 98 & 68 & 12 & 178 \\
\hline 119 & 1208 & 395 & 325 & 84 & 804 \\
\hline Total & & & & & \\
\hline
\end{tabular}

Table 39. Available periods from controlled releases for various filters.

\begin{tabular}{|l|c|c|}
\hline Period Attribute & Background & Release \\
\hline Total Possible & \multicolumn{2}{|c|}{10,878} \\
\hline Valid EC and OTM & 4599 & 2684 \\
\hline Background Pre-Release & 1503 & - \\
\hline Background Post-Release & 3124 & - \\
\hline $\pm 45^{\circ}$ Wind Direction (Valid Set) & 1208 & 804 \\
\hline Location: 2 & 283 & 382 \\
\hline Location: 3 & 430 & 244 \\
\hline Location: 4 & 98 & 178 \\
\hline Distance: $42 \mathrm{~m}$ & 577 & 382 \\
\hline Distance: $57 \mathrm{~m}$ & 224 & 47 \\
\hline Distance: $72 \mathrm{~m}$ & 289 & 197 \\
\hline Distance: $119 \mathrm{~m}$ & 118 & 178 \\
\hline QC CH4 Flux $<2$ & 891 & 608 \\
\hline OTM DQI $<10$ & 76 & 203 \\
\hline Daytime (EddyPro $\mathbb{~})$ & 711 & 493 \\
\hline
\end{tabular}


Table 40. Statistics of valid release periods.

\begin{tabular}{|c|c|c|c|c|c|c|}
\hline Variable & $\begin{array}{c}\text { Valid } \\
\text { Count } \\
(\#)\end{array}$ & Mean & Std. Dev. & Min & Median & Max \\
\hline Wind Speed $(\mathrm{m} / \mathrm{s})$ & 804 & 1.6 & 1.2 & 0.0 & 1.5 & 5.3 \\
\hline Max Wind Speed $(\mathrm{m} / \mathrm{s})$ & 804 & 3.8 & 2.6 & 0.2 & 3.7 & 14.0 \\
\hline $\mathrm{u}^{*}(\mathrm{~m} / \mathrm{s})$ & 804 & 0.19 & 0.15 & 0.002 & 0.17 & 1.02 \\
\hline $\operatorname{TKE}\left(\mathrm{m}^{2} / \mathrm{s}^{2}\right)$ & 804 & 0.56 & 0.56 & 0.006 & 0.40 & 3.41 \\
\hline Mean Temperature $\left({ }^{\circ} \mathrm{C}\right)$ & 804 & 22.5 & 5.4 & 8.0 & 23.3 & 31.9 \\
\hline Mean Pressure $(\mathrm{kPa})$ & 804 & 95.8 & 0.3 & 95.1 & 95.8 & 96.6 \\
\hline Relative Humidity (\%) & 804 & 70.7 & 19.9 & 36.5 & 64.7 & 98.6 \\
\hline $\mathrm{VPD}(\mathrm{kPa})$ & 619 & 1.14 & 0.79 & 0.00 & 1.19 & 2.75 \\
\hline Air Heat Capacity (kJ/K-kg) & 804 & 1.01 & 0.006 & 0.97 & 1.02 & 1.02 \\
\hline Solar Loading $\left(\mathrm{W} / \mathrm{m}^{2}\right)$ & 804 & 338 & 348 & 0 & 225 & 1102 \\
\hline $\mathrm{CH}_{4}$ Concentration (ppm) & 804 & 2.32 & 0.48 & 1.87 & 2.13 & 6.26 \\
\hline $\mathrm{CO}_{2}$ Concentration (ppm) & 649 & 460 & 132 & 292 & 397 & 1092 \\
\hline $\mathrm{H}_{2} \mathrm{O}$ Concentration (ppm) & 649 & 19657 & 8595 & 420 & 19666 & 148480 \\
\hline $\mathrm{CH}_{4}$ Background Concentration (ppm) & 804 & 2.17 & 0.32 & 1.81 & 2.05 & 4.00 \\
\hline Sigma Y & 804 & 10.1 & 5.5 & 3.7 & 8.7 & 26.7 \\
\hline Sigma Z & 804 & 5.8 & 3.1 & 2.2 & 4.7 & 14.7 \\
\hline TI & 804 & 0.138 & 0.057 & 0.041 & 0.129 & 0.595 \\
\hline ASI Class & 804 & 5 & 1.2 & 2 & 5 & 7 \\
\hline Signal Strength LI-7500 (\%) & 648 & 80.2 & 23.6 & 0.1 & 90.2 & 90.9 \\
\hline RSSI LI-7700 (\%) & 804 & 31.0 & 13.8 & 11.1 & 26.1 & 72.8 \\
\hline
\end{tabular}

\subsection{OTM Improvement}

To determine whether OTM could be made more accurate, an analysis was performed to determine the optimal binning parameters, defined in Table 31. It is unclear whether these parameters were examined during the development of the method. Manipulation of the parameters does not affect the theory of OTM, but simply changes the binning of the data, resulting in potentially different horizontal flux calculations. To find the optimal set of binning parameters a Taguchi design array was utilized.

Taguchi design of experiments utilize fractional factorial designs which allow for analysis of several factors (binning parameters in this case) on several levels (different values of each binning parameter) to find a combination of factors that minimizes or maximizes the value used to measure performance of the system. In this case the value used to measure the performance of the system was a weighted root mean square error (RMSE) of the OTM release rate estimation which had units of $(\mathrm{g} / \mathrm{s})$ and was meant to be minimized. Taguchi methods are based on orthogonal design arrays. An orthogonal array is "a matrix whose columns have the property that in every pair of columns each of the possible ordered pairs of elements appears the same number of times" [137]. The matrices of orthogonal arrays allow the different levels of each factor to be combined with each level of another factor. This method of design allows for analysis of each variable individually without it being weighted by some combination of other variables used in the analysis. To determine the optimal levels of each factor a level average is used to quantify the factor levels impact on the metric of analysis. 
A Taguchi orthogonal array was used to optimize the binning parameters of OTM by minimizing the weighted RMSE of the controlled release dataset. Each of the OTM binning parameters $(\mathrm{P}=5)$ were evaluated at four different levels $(\mathrm{L}=4)$. Notation in Taguchi arrays is often defined by the number of levels raised to the number of factors in the experiment. For example, a design with 5 factors, each evaluated at 4 levels would be noted as $\left(4^{5}\right)$. This represents the number of total combinations required for unique analyses of all factors and levels (1024). However, with the use of a Taguchi design array the number of analyses required can be significantly reduced, saving an immense amount of computational time. The analysis of the OTM parameters utilized a Taguchi orthogonal array structure known as L16. The design accepts five factors with four levels each and requires only 16 separate experimental analyses. The L16 design matrix is presented in Table 41.

Table 41. Taguchi design array for OTM parameter optimization.

\begin{tabular}{|c|c|c|c|c|c|}
\hline $\begin{array}{c}\text { Analysis } \\
\#\end{array}$ & $\begin{array}{c}\text { Bin } \\
\left.\text { Size ( }{ }^{\circ}\right)\end{array}$ & $\begin{array}{c}\text { Wind } \\
\text { Speed } \\
\text { Limit } \\
(\mathbf{m} / \mathbf{s})\end{array}$ & $\begin{array}{c}\text { Bin Angle } \\
\text { Cut Limit } \\
\left({ }^{\circ}\right)\end{array}$ & $\begin{array}{c}\text { Bin Percent } \\
\text { Cut Limit } \\
\mathbf{( \% )}\end{array}$ & $\begin{array}{c}\text { Background } \\
\text { Percent (\%) }\end{array}$ \\
\hline 1 & 5 & 0 & 30 & 0 & 3 \\
\hline 2 & 5 & 0.5 & 45 & 1 & 5 \\
\hline 3 & 5 & 1 & 60 & 2 & 7 \\
\hline 4 & 5 & 2 & 75 & 3 & 10 \\
\hline 5 & 10 & 0 & 45 & 2 & 10 \\
\hline 6 & 10 & 0.5 & 30 & 3 & 7 \\
\hline 7 & 10 & 1 & 75 & 0 & 5 \\
\hline 8 & 10 & 2 & 60 & 1 & 3 \\
\hline 9 & 15 & 0 & 60 & 3 & 5 \\
\hline 10 & 15 & 0.5 & 75 & 2 & 3 \\
\hline 11 & 15 & 1 & 30 & 1 & 10 \\
\hline 12 & 15 & 2 & 45 & 0 & 7 \\
\hline 13 & 20 & 0 & 75 & 1 & 7 \\
\hline 14 & 20 & 0.5 & 60 & 0 & 10 \\
\hline 15 & 20 & 1 & 45 & 3 & 3 \\
\hline 16 & 20 & 2 & 30 & 2 & 5 \\
\hline & & & & & \\
\hline
\end{tabular}

For each of the analyses 100 random periods were selected from the possible $804 \mathrm{WF}$ periods. OTM calculations were then performed on the 100 random periods. The same 100 periods were not selected for each of the Taguchi runs to avoid bias in the selection such as high or low wind speed. Not all Taguchi options resulted in 100 valid estimates. To emphasize the importance of successful calculations, which resulted in more usable period estimations, the RMSE of each parameter analysis was weighted. Those analyses that produced more periods were given higher preference by creating a weighting factor proportional to the number of successful calculations. This method emphasized a low RMSE and a high number of successful calculations. RMSE was used as the primary evaluation metric throughout this work for several reasons. It has the same units as the metric being evaluated, unlike mean squared error. Another common metric, mean absolute error, also has the same units of the evaluated variables, however, RMSE places a larger emphasis on outliers which were thought to be particularly undesirable in this research. The formula for RMSE is represented by Equation 19. The weighted RMSE is presented in Equation 20. 


$$
\begin{gathered}
\operatorname{RMSE}(g / s)=\sqrt{\sum_{i=1}^{n} \frac{\left(y_{i}-\hat{y}\right)^{2}}{n}} \\
\text { Weighted } R M S E=\frac{(100-\# \text { of successful calculations })}{100} * \text { RMSE }
\end{gathered}
$$

Equation 19

Equation 20

To ensure that the weighted RMSEs were not strongly correlated with the distribution of release rates and distances, the mean distance and mean release rate of each analysis were also calculated. Correlations between variables were calculated in Python using the SciPy [138] library's Pearson correlation coefficient, presented in Equation 21. The function also provides a two-tailed p-value. P-values were compared to a significance level $(\alpha)$ of 0.05 to test for significance. Neither the mean distance nor the mean release rate had an absolute Pearson correlation coefficient greater than 0.2 or a p-value less than the significance level indicating that there was $95 \%$ confidence that the variables were not correlated with the weighted RMSE. The results of the L16 Taguchi analysis are presented in Table 42. The level average weighted RMSE of each of the parameters evaluated are presented in Table 43.

$$
\text { Pearson } r=\frac{\sum\left(x_{i}-\hat{x}\right) \sum\left(y_{i}-\hat{y}\right)}{\sqrt{\sum\left(x_{i}-\hat{x}\right)^{2} \sum\left(y_{i}-\hat{y}\right)^{2}}}
$$

Equation 21

Table 42. Results of Taguchi design array analysis.

\begin{tabular}{|c|c|c|c|c|c|}
\hline $\begin{array}{c}\text { Analysis } \\
\#\end{array}$ & $\begin{array}{c}\text { Successful } \\
\text { Calculations } \\
\mathbf{( \# )}\end{array}$ & $\begin{array}{c}\text { RMSE } \\
\mathbf{( g / s )}\end{array}$ & $\begin{array}{c}\text { Weighted } \\
\text { RMSE } \\
\mathbf{( g / \mathbf { s } )}\end{array}$ & $\begin{array}{c}\text { Mean } \\
\text { Release } \\
\text { Rate } \\
\mathbf{( g / \mathbf { s } )}\end{array}$ & $\begin{array}{c}\text { Mean } \\
\text { Distance } \\
\mathbf{( m )}\end{array}$ \\
\hline 1 & 97 & 0.089 & 0.0027 & 0.095 & 67.2 \\
\hline 2 & 93 & 0.091 & 0.0064 & 0.093 & 60.8 \\
\hline 3 & 80 & 0.109 & 0.0219 & 0.091 & 70.7 \\
\hline 4 & 62 & 0.110 & 0.0419 & 0.100 & 67.1 \\
\hline 5 & 97 & 0.086 & 0.0026 & 0.092 & 70.0 \\
\hline 6 & 92 & 0.094 & 0.0075 & 0.087 & 66.6 \\
\hline 7 & 78 & 0.127 & 0.0280 & 0.081 & 69.7 \\
\hline 8 & 61 & 0.105 & 0.0408 & 0.097 & 62.8 \\
\hline 9 & 98 & 0.092 & 0.0018 & 0.093 & 61.2 \\
\hline 10 & 92 & 0.079 & 0.0063 & 0.074 & 64.7 \\
\hline 11 & 68 & 0.157 & 0.0504 & 0.086 & 68.1 \\
\hline 12 & 53 & 0.072 & 0.0339 & 0.081 & 64.5 \\
\hline 13 & 98 & 0.096 & 0.0019 & 0.095 & 69.4 \\
\hline 14 & 85 & 0.101 & 0.0152 & 0.087 & 65.4 \\
\hline 15 & 65 & 0.084 & 0.0293 & 0.091 & 70.2 \\
\hline 16 & 31 & 0.066 & 0.0453 & 0.084 & 66.6 \\
\hline
\end{tabular}


Table 43. Level average OTM parameters from Taguchi design array.

\begin{tabular}{|c|c|c|}
\hline Parameter & Level Value & $\begin{array}{c}\text { Level Average } \\
\text { Weighted RMSE (g/s) }\end{array}$ \\
\hline \multirow{4}{*}{ Bin Size () } & 5 & 0.0182 \\
\hline & 10 & 0.0197 \\
\hline & 15 & 0.0231 \\
\hline & 20 & 0.0229 \\
\hline \multirow{4}{*}{ Wind Speed Limit $(\mathrm{m} / \mathrm{s})$} & 0 & 0.0023 \\
\hline & 0.5 & 0.0088 \\
\hline & 1 & 0.0324 \\
\hline & 2 & 0.0405 \\
\hline \multirow{4}{*}{ Bin Angle Limit $\left({ }^{\circ}\right)$} & 30 & 0.0265 \\
\hline & 45 & 0.0180 \\
\hline & 60 & 0.0199 \\
\hline & 75 & 0.0195 \\
\hline \multirow{4}{*}{ Bin Percent Limit (\%) } & 0 & 0.0199 \\
\hline & 1 & 0.0249 \\
\hline & 2 & 0.0190 \\
\hline & 3 & 0.0201 \\
\hline \multirow{4}{*}{ Background Percent (\%) } & 3 & 0.0198 \\
\hline & 5 & 0.0204 \\
\hline & 7 & 0.0163 \\
\hline & 10 & 0.0275 \\
\hline
\end{tabular}

Based on the level average RMSE the optimal settings were a "Bin Size" of $5^{\circ}$, a "Wind Speed Limit" of 0 $\mathrm{m} / \mathrm{s}$, a "Bin Angle Limit" of $45^{\circ}$, a "Bin Percent Limit" of 2\%, and a "Background Percent" of 7\%. These bin parameters were used to calculate the OTM estimates for all available farm periods. The Taguchi OTM estimates were compared to the default OTM estimates to ensure the Taguchi OTM results improved the RMSE across the entire dataset consisting of 2012 total periods (804 with controlled release, 1208 background). The RMSE results are presented in Table 44. The Taguchi OTM parameters were also used to calculate the results of the default EPA dataset; however, the results of this dataset were not improved by the Taguchi method, resulting in an $8 \%$ increase in RMSE compared to the default OTM results. The results of the controlled release dataset may be influenced by the large number of smaller releases $(0.036$ $\mathrm{g} / \mathrm{s}$ ), which the Taguchi method improved most significantly. To ensure that the Taguchi method did not limit improvement to a specific release rate or distance, the complete RRD matrix RMSE is presented in Table 45. The percent improvement over the default dataset is presented in Table 46. The standard deviation of measurements was also reduced across all RRD scenarios. This lends more confidence to the repeatability of the Taguchi OTM results applied to the controlled releases. The percent reduction in the standard deviation of each RRD scenario is presented in Table 47. 
Table 44. Taguchi and default OTM results.

\begin{tabular}{|c|c|c|c|}
\hline \multirow{2}{*}{ Bin Parameters } & \multicolumn{3}{|c|}{ RMSE (g/s) } \\
\cline { 2 - 4 } & Background & Release & All \\
\hline Default & 0.045 & 0.151 & 0.102 \\
\hline Taguchi & 0.033 & 0.123 & 0.082 \\
\hline
\end{tabular}

Table 45. Taguchi versus default OTM RMSE, across all controlled release scenarios.

\begin{tabular}{|c|l|c|c|c|c|}
\hline \multirow{2}{*}{$\begin{array}{c}\text { Release } \\
\text { Rate (g/s) }\end{array}$} & \multirow{2}{*}{ Parameters } & \multicolumn{5}{|c|}{ Release Distance (m) } \\
\cline { 3 - 6 } $\mathbf{0}$ & Taguchi & 0.023 & 0.029 & 0.027 & 0.071 \\
\cline { 2 - 6 } & Default & 0.040 & 0.037 & 0.039 & 0.082 \\
\hline \multirow{2}{*}{$\mathbf{0 . 0 3 6}$} & Taguchi & 0.022 & - & 0.027 & 0.065 \\
\cline { 2 - 6 } & Default & 0.023 & - & 0.063 & 0.101 \\
\hline \multirow{2}{*}{$\mathbf{0 . 1 1 9}$} & Taguchi & 0.092 & 0.084 & 0.091 & 0.327 \\
\cline { 2 - 6 } & Default & 0.093 & 0.091 & 0.089 & 0.376 \\
\hline \multirow{2}{*}{$\mathbf{0 . 2 3 9}$} & Taguchi & 0.176 & - & 0.159 & 0.157 \\
\cline { 2 - 6 } & Default & 0.185 & - & 0.309 & 0.240 \\
\hline
\end{tabular}

Table 46. Reduced OTM RMSE by Taguchi optimization.

\begin{tabular}{|c|c|c|c|c|}
\hline \multirow{2}{*}{$\begin{array}{c}\text { Release Rate } \\
(\mathbf{g} / \mathbf{s})\end{array}$} & \multicolumn{4}{|c|}{ Release Distance (m) } \\
\cline { 2 - 5 } $\mathbf{0}$ & $\mathbf{4 2}$ & $\mathbf{5 7}$ & $\mathbf{7 2}$ & $\mathbf{1 1 9}$ \\
\hline $\mathbf{0 . 0 3 6}$ & $43 \%$ & $22 \%$ & $31 \%$ & $13 \%$ \\
\hline $\mathbf{0 . 1 1 9}$ & $1 \%$ & - & $57 \%$ & $36 \%$ \\
\hline $\mathbf{0 . 2 3 9}$ & $5 \%$ & $8 \%$ & $-2 \%$ & $13 \%$ \\
\hline
\end{tabular}

Table 47. Reduced OTM standard deviation by Taguchi optimization.

\begin{tabular}{|c|c|c|c|c|}
\hline \multirow{2}{*}{$\begin{array}{c}\text { Release Rate } \\
(\mathbf{g} / \mathbf{s})\end{array}$} & \multicolumn{4}{|c|}{ Release Distance (m) } \\
\cline { 2 - 5 } $\mathbf{0}$ & $\mathbf{4 2}$ & $\mathbf{5 7}$ & $\mathbf{7 2}$ & $\mathbf{1 1 9}$ \\
\hline $\mathbf{0 . 0 3 6}$ & $23 \%$ & $20 \%$ & $36 \%$ & $17 \%$ \\
\hline $\mathbf{0 . 1 1 9}$ & $26 \%$ & - & $60 \%$ & $35 \%$ \\
\hline $\mathbf{0 . 2 3 9}$ & $38 \%$ & - & $5 \% \%$ & $29 \%$ \\
\hline
\end{tabular}




\subsection{EC Footprint Analysis}

For comparison with the controlled releases and OTM estimations, a methane mass emission rate was required from the EC method. The units of the EddyPro ${ }^{\circledR}$ calculated methane flux were micromoles per meter squared per second $\left(\mu \mathrm{mol} / \mathrm{m}^{2} \mathrm{~s}\right)$. To obtain a mass flux estimate from these values some source area must be utilized, and the molar concentrations must be converted to mass. As discussed previously many studies have examined different ways to calculate the flux footprint which defines a source area. Combining the flux footprint with the EddyPro® calculation of methane flux allowed for an estimation of the methane mass emission rate.

By defining a footprint area, several researchers have estimated point source emissions from flux footprints using models as previously discussed. The two most used models for estimating the flux footprint are those developed by Kormann and Meixner (KM) and the flux footprint parameterization (FFP) by Kljun et al [75], [76]. These footprint models were both utilized to estimate a flux footprint which produced a grid of source attribution as a percentage of total contribution to the flux. Research on such models has been performed previously and model source codes were obtained for both the FFP and KM models online from OzFlux which is a well-established part of the international network FluxNet [139]. OzFlux developed versions of the FFP and KM models for Python which were available on GitHub [140]. These functions were used for estimation of the flux footprints of the valid controlled release experiments. The footprint models required the inputs listed in Table 48.

Table 48. Footprint function inputs for OzFlux developed functions.

\begin{tabular}{|c|c|c|c|c|l|}
\hline Variable & Units & Description & FFP & KM & \multicolumn{1}{|c|}{ Source } \\
\hline $\mathrm{z}_{\mathrm{m}}$ & $\mathrm{m}$ & Measurement height above displacement height & $\mathrm{x}$ & $\mathrm{x}$ & $\begin{array}{l}\text { Fixed (Defined as } \\
4.0 \mathrm{~m} \text { ) }\end{array}$ \\
\hline $\mathrm{u}_{\mathrm{mean}}$ & $\mathrm{m} / \mathrm{s}$ & Mean wind speed at $\mathrm{z}_{\mathrm{m}}$ & $\mathrm{x}$ & $\mathrm{x}$ & EddyPro Output \\
\hline $\mathrm{h}$ & $\mathrm{m}$ & boundary layer height & $\mathrm{x}$ & & $\begin{array}{l}\text { Calculated based on } \\
\text { Dumortier et al.[67] }\end{array}$ \\
\hline $\mathrm{ol}$ & $\mathrm{m}$ & Obukhov length & $\mathrm{x}$ & $\mathrm{x}$ & $\begin{array}{l}\text { Calculated based on } \\
\text { Kljun et al. [76] }\end{array}$ \\
\hline$\sigma_{\mathrm{v}}$ & $\mathrm{m} / \mathrm{s}$ & standard deviation of lateral velocity fluctuations & $\mathrm{x}$ & $\mathrm{x}$ & EddyPro Output \\
\hline $\mathrm{u}^{*}$ & $\mathrm{~m} / \mathrm{s}$ & friction velocity & $\mathrm{x}$ & $\mathrm{x}$ & EddyPro Output \\
\hline wind_dir & $\circ$ & wind direction in degrees for rotation of the footprint & $\mathrm{x}$ & $\mathrm{x}$ & EddyPro Output \\
\hline domain & $\mathrm{m} * \mathrm{~m}$ & Size of the source area to calculate & $\mathrm{x}$ & $\mathrm{x}$ & $\begin{array}{l}\text { Defined as (300 m } \\
\text { by 300 m) }\end{array}$ \\
\hline
\end{tabular}

The domain defined was $300 \mathrm{~m}$ by $300 \mathrm{~m}$ area with the tower location being fixed at the origin. This resulted in a "view" of the footprint contributions that stretched from the tower $150 \mathrm{~m}$ in four directions. This domain size allowed all controlled releases to fit within the bounds. The number of cells within the grid in the $\mathrm{x}$ and y direction were 300. This produced individual grid sizes of $1 \mathrm{~m}$ by $1 \mathrm{~m}$. For each period, both functions output a two-dimensional normalized footprint function $\left(1 / \mathrm{m}^{2}\right)$ matrix. Because the location of the controlled release within the grid was known the normalized value of the source area $(\varphi)$ could be calculated for the location on the grid which contained the source. This approach was used by Dumortier et al. (2019) and Stoy et al. (2021) to estimate point source emissions from controlled releases of methane and bison, respectively [66], [67].

In addition to the primary filters used to reduce the number of valid release periods to 804 . Footprint specific filters were applied. These included those defined by Equation 9 and Equation 10 for the FFP model and a $\mathrm{u}^{*}$ greater than $0.1 \mathrm{~m} / \mathrm{s}$ as defined by the KM model. In addition to these filters, the EC quality control filter for methane flux was used to eliminate periods with a value of two based on the method of Mauder and 
Foken [85]. Periods that resulted in a negative estimate of methane flux were also eliminated from the analysis. A negative flux indicates that methane is being absorbed by the ecosystem. Clearly this indicates an erroneous measurement when there is a known mass rate emission source within the footprint. It was therefore assumed that the footprints from the periods with a negative methane flux did not include the location of the point source.

After the application of these filters, the total number of periods available for analysis were 304 . The footprint functions were applied to these 304 periods. The footprint functions produced a wide range of values. To combine the normalized footprint function with the flux estimate and determine a mass emission rate Equation 22 was utilized.

$$
\begin{aligned}
\mathrm{CH}_{4}(\mathrm{~g} / \mathrm{s})= & {\left[\text { ch4_flux }\left(\frac{\mu \mathrm{mol}}{\mathrm{m}^{2} \mathrm{~s}}\right)\right] *\left(\frac{1 \mathrm{~mol}}{10^{6} \mu \mathrm{mol}}\right) *\left(\frac{16.04 \mathrm{~g}}{\mathrm{~mol}}\right) } \\
& *\left[\frac{1}{\varphi}\left(\mathrm{m}^{2}\right)\right]
\end{aligned}
$$

Equation 22

Only periods that produced results for both methods were utilized for comparison. Periods were eliminated from the analysis if either method resulted in infinite values $(n=53)$. This left only 251 periods for comparison. The RMSE of these periods for each of the estimates from the footprint function when compared to the known release rates were both larger than $1 \times 10^{6}(\mathrm{~g} / \mathrm{s})$. This was clearly the result of several near-infinite values being included in the analysis. To eliminate these erroneous periods, a modified $\mathrm{z}$-score was used to remove potential outliers. If either of the methods resulted in an outlier, the period was discarded. The total number of periods after both sets of outliers were removed was 142. The RMSEs of these datasets were $0.079 \mathrm{~g} / \mathrm{s}$ for the KM model and $0.087 \mathrm{~g} / \mathrm{s}$ for the FFP model. These RMSEs were on the same order of magnitude as the controlled releases and the RMSEs obtained from OTM. An example of footprint results for a single period in the form of heatmap are presented in Figure 29 with the corresponding release rate and tower and release location. The KM model performed better in terms of RMSE when each period was evaluated individually, however, another way to quantify emissions was via grouping similar periods together. This averaging approach was used by Dumortier et al (2019) to estimate point source emissions.
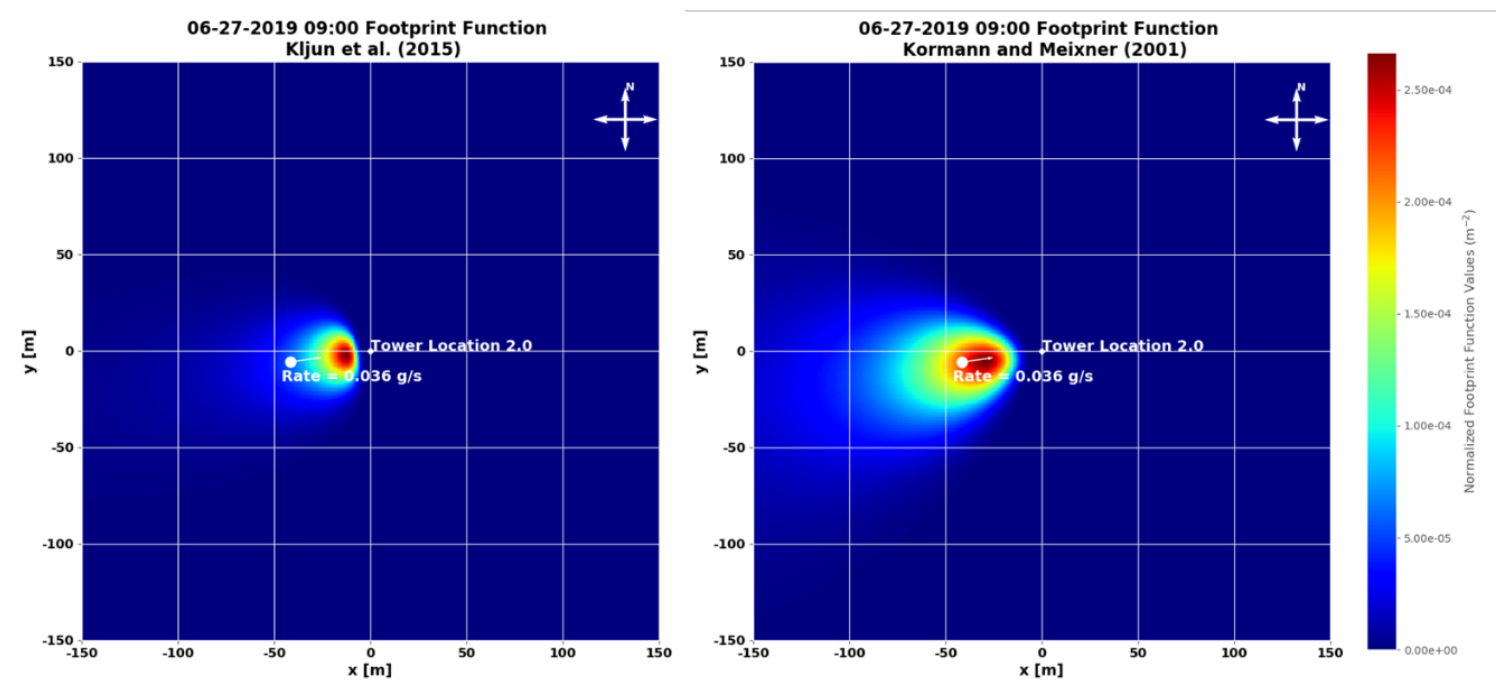

Figure 29. Example EC footprints of FFP and KM, during controlled releases.

Dumortier et al. presented a method for estimating the mass emission rates of a series of periods by using a parity plot of the estimated flux footprint contribution of the periods in $\left[\mathrm{m}^{2}\right]$ against the methane flux in 
units of mass emission rate per unit area $\left[\mathrm{g} / \mathrm{m}^{2} \mathrm{~s}\right]$. The slope of the linear approximation estimated the mass emission rate of the entire time interval which spanned multiple periods. These results were estimated for release rate and distance combinations if the number of available periods was greater than 10 . Only the periods with a release distance of $42 \mathrm{~m}$ resulted in more than 10 estimations and the results of these estimations are presented in Table 49. Only three other periods were not eliminated by the various filters used or did not produce erroneous or outlier estimations. The results display a tendency of both methods to significantly underestimate emissions rates. The FFP results did produce slightly better results across analyzed releases when using this method. An example of the parity plot such as those used in Dumortier et al. is presented by Figure 30 .

Table 49. Dumortier et al. method results.

\begin{tabular}{|c|c|c|c|c|c|c|c|}
\hline \multirow{2}{*}{$\begin{array}{c}\text { Actual } \\
(\mathbf{g} / \mathbf{s})\end{array}$} & \multirow{2}{*}{$\begin{array}{c}\text { Periods } \\
(\#)\end{array}$} & $\begin{array}{c}\text { Slope } \\
(\mathbf{g} / \mathbf{s})\end{array}$ & $\begin{array}{c}\text { \% of } \\
\text { True }\end{array}$ & $\mathbf{R}^{\mathbf{2}}$ & $\begin{array}{c}\text { Slope } \\
(\mathbf{g} / \mathbf{s})\end{array}$ & $\begin{array}{c}\text { \% of } \\
\text { True }\end{array}$ & $\mathbf{R}^{\mathbf{2}}$ \\
\hline 0.036 & 87 & 0.006 & $16 \%$ & 0.246 & 0.008 & $22 \%$ & 0.206 \\
\hline 0.119 & 38 & 0.011 & $9 \%$ & 0.460 & 0.013 & $11 \%$ & 0.342 \\
\hline 0.239 & 14 & 0.015 & $6 \%$ & 0.133 & 0.035 & $15 \%$ & 0.243 \\
\hline
\end{tabular}

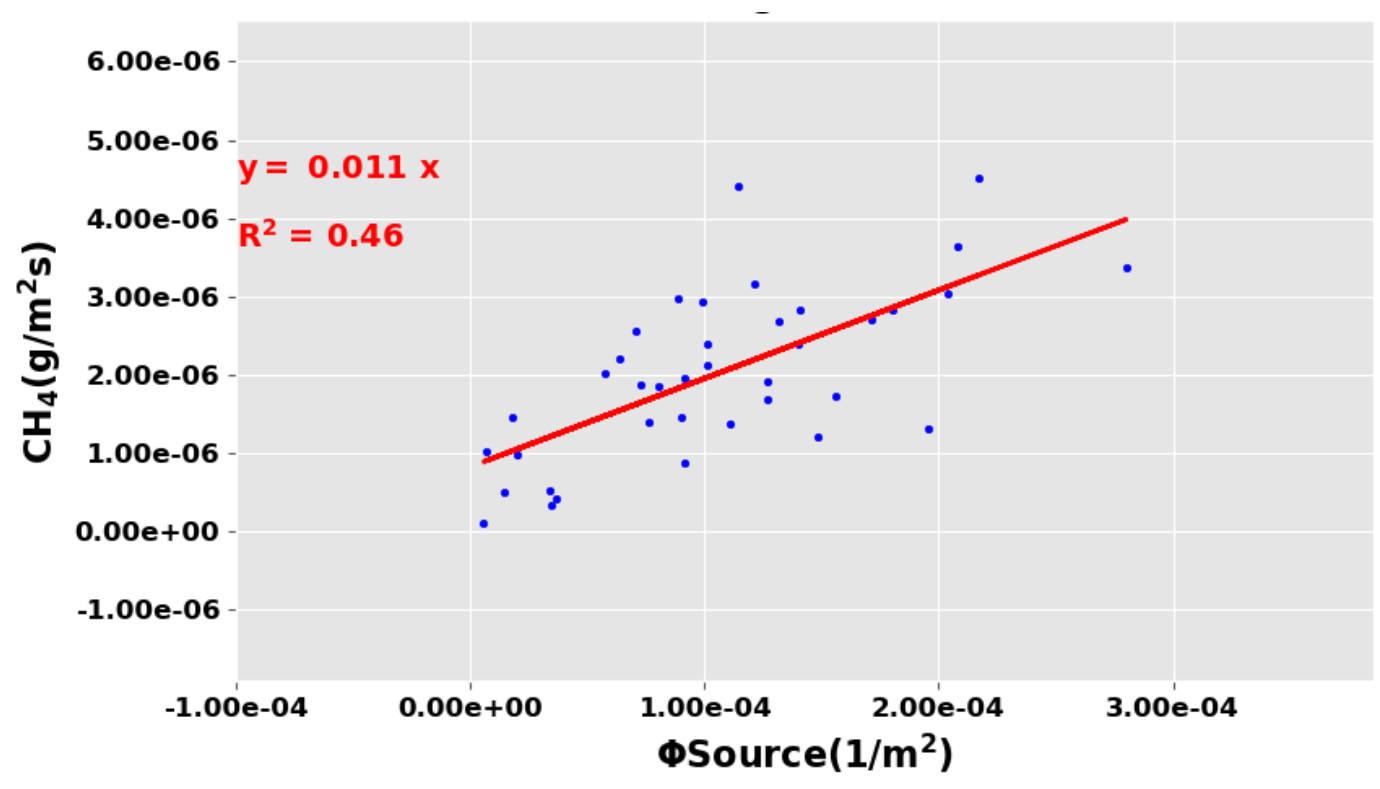

Figure 30. Example of Dumortier et al. method.

\subsection{Method Uncertainty}

To better understand the methods used, some uncertainty estimations were performed. Previous research efforts have quantified the uncertainty of OTM as a percentage of the known release.

\subsubsection{OTM Measurement Uncertainty}

There was no research previously completed on how to assess the instrument impact on the uncertainty of OTM calculations. Historically OTM uncertainty has been reported as a range of errors as a percentage of 
estimates compared to known releases. When compared to controlled releases without DQI filtering errors in the initial test set ranged from $-84 \%$ to $184 \%$ of the estimate. With DQI filtering the errors ranged from $-60 \%$ to $52 \%$ [48]. A second controlled release comparison found that data had a $2 \sigma$ error of $\pm 56 \%$ [ 51$]$. A study that combined most of the published controlled releases concluded that OTM typically underestimated by $10 \%$ overall but had a propensity to overestimate small releases. The authors stated that one could generally expect a $2 \sigma$ error of $\pm 70 \%[53]$.

These types of error estimates provide little information about the universal uncertainty inherent to the method. The errors could be specific to the test set in question. Without a sufficient sample size encompassing estimates over a range of micrometeorological conditions and leak scenarios it is difficult to determine an uncertainty value that could be used for the method universally. is difficult to say what the true uncertainty of the method. To determine a minimum measurement uncertainty (not estimate uncertainty) for a given sampling period, the measurement limitations of the devices were considered. State of the art equipment were used during data collection (as described in 3.1.1), but the instruments used still had some level of measurement uncertainty. The instrument limitations as published by the manufacturers were used to determine individual measurement uncertainties. The key variables used for OTM calculations were 3-D wind speeds, methane concentration, temperature, and pressure. The instruments used to measure these variables and their uncertainties are presented in Table 50.

Table 50. Relevant OTM instrumentation information.

\begin{tabular}{|c|c|c|c|}
\hline Device & $\begin{array}{l}\text { Relevant } \\
\text { Variable }\end{array}$ & Resolution & Accuracy \\
\hline \multirow{3}{*}{$\begin{array}{c}\text { Gill } \\
\text { WindMaster }\end{array}$} & WS_X & \multirow{3}{*}{ $\pm 0.01 \mathrm{~m} / \mathrm{s}$} & \multirow{3}{*}{$<1.5 \%$ RMS } \\
\hline & ws_y & & \\
\hline & WS_Z & & \\
\hline LI-7700 & $\operatorname{ch} 4$ conc & $\pm 0.005 \mathrm{ppm}$ & $<1 \%$ \\
\hline \multirow{2}{*}{ LI-7500 } & $\mathrm{T}$ & $\pm 0.003 \mathrm{~K}$ & $\pm 0.3 \mathrm{~K}$ \\
\hline & $\mathrm{P}$ & $\pm 0.06 \mathrm{mbar}$ & \pm 4 mbar \\
\hline
\end{tabular}

Total uncertainties of each individual data point collected at $10 \mathrm{~Hz}$ were determined from the resolution and accuracy of the respective instrument. The accuracy uncertainty was determined based on the interval within which the true value was known. If the accuracy of a device was presented as a percentage of the measurement, the actual value was assumed to fall within the range of the [measured value $+/$ - the accuracy times the measured value]. If the accuracy was presented as a standard unit, the actual value was assumed to fall within the range of the [measured value $+/-$ the unit value]. Regardless of how the accuracy was reported by the device manufacturer, the standard uncertainty due to accuracy was calculated as the half interval divided by the square root of three. This calculation was derived from the assumption that the accuracy of the device acts as a rectangular distribution. In other words, all possible values within the accuracy range are equally likely for each measurement point [141]. Examples of the individual measurement uncertainties due to accuracy are presented below for methane (as a percentage) and temperature (as a unit value).

The LI-7700 has a methane concentration accuracy of $\pm 1 \%$ of reading. For example, if the reading was $2.0945 \mathrm{ppm}$. The standard uncertainty due to accuracy would be:

$$
\mathrm{CH}_{4} \text { accuracy }_{\text {uncertainty }}=\frac{(2.0945 \mathrm{ppm}) * 0.01}{2 \sqrt{3}}= \pm 0.00605 \mathrm{ppm}
$$


The accuracy of the temperature measurement of the LI-7500 is $\pm 0.3{ }^{\circ} \mathrm{C}$. The standard uncertainty of any temperature measurement would be:

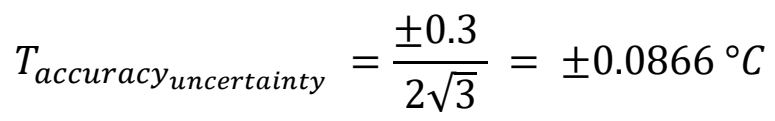

The instruments used for measurement also produced uncertainties due to their resolution. Since any digital instrument has a discrete value due to rounding, the true value could lie within \pm 0.5 times the resolution of the display. To determine the standard uncertainty of the device due to its resolution the resolutions reported were divided by the square root of three (again assuming that each possible value in the half interval were equally likely to occur) [142]. For example, the resolution of the WindMaster is listed as $0.01 \mathrm{~m} / \mathrm{s}$ for each of the coordinate directions of wind speed, so the standard uncertainty due to the resolution is calculated as follows:

$$
W S_{\text {resolution }} \text { uncertainty }=\frac{ \pm 0.01 \mathrm{~m} / \mathrm{s}}{\sqrt{3}}= \pm 0.00577 \mathrm{~m} / \mathrm{s}
$$

The standard uncertainty of each individual measurement could be calculated by combining the resolution uncertainty and accuracy uncertainty [143]. The total standard uncertainty of each individual measurement point was calculated using Equation 23 for each variable.

$$
\begin{aligned}
& \text { total }_{\text {standard }} \text { uncertainty } \\
& =\sqrt{\text { resolution }_{\text {uncertainty }}^{2}+\text { accuracy }_{\text {uncertainty }}^{2}}
\end{aligned}
$$

Equation 23

A complete example for calculating the standard uncertainty in a methane concentration is presented below.

1. The resolution of the LI-7700 is 5 parts per billion (ppb). The half interval in ppm is then 0.005 .

$$
\text { resolution }_{\text {uncertainty }}=\frac{ \pm 0.005}{\sqrt{3}}= \pm 0.0029 \mathrm{ppm}
$$

2. The accuracy of the analyzer is $1 \%$ of the reading across the full calibration range. So, if the concentration is $2.0945 \mathrm{ppm}$.

$$
\text { accuracy }_{\text {uncertainty }}=\frac{2.0945 * 0.01}{2 \sqrt{3}}= \pm 0.0061 \mathrm{ppm}
$$

3. The total uncertainty is the sum of the squares of the resolution and accuracy uncertainty.

$$
\begin{gathered}
\text { total }_{\text {uncertainty }}=\sqrt{ \pm 0.0029^{2}+ \pm 0.0061^{2}}= \pm 0.0068 \mathrm{ppm} \\
\mathrm{CH}_{4}=2.0945 \pm 0.0068 \mathrm{ppm}
\end{gathered}
$$

To determine the standard measurement uncertainty of the OTM periods, total standard uncertainty of each measurement from each device was calculated. So, for each individual point (10 Hz data) the uncertainty of that measurement could be quantified. This resulted in an "upper", "lower", and "measured" value for each variable and each data point. There were six $10 \mathrm{~Hz}$ variables used in the calculation $(c h 4, u, v, w, t$, $p$ ). The equations below describe the three levels:

measured value $=$ the value reported and recorded form the instrument 


$$
\begin{aligned}
& \text { upper value }=\text { measured value }+ \text { standard uncertainty of measured value } \\
& \text { lower value }=\text { measured value }- \text { standard uncertainty of measured value }
\end{aligned}
$$

To analyze the uncertainty of an OTM averaging period estimate, a complete analysis of all the combinations of maximum measurement uncertainty were applied to each point in that period. Within a given 15-minute period there could be up to 9000 data points recorded for each variable which could exhibit the full spectrum of measurement uncertainty. For simplicity it was assumed that the true value of every point of a given variable was either the "upper", "lower", or "measured" value. Applying this simplifying assumption to all six variables gave a total of 729 potential OTM estimates for each period based on the number of variables $(m=6)$ and number of potential values $(n=3)$, such that:

$$
n^{m}=3^{6}=729
$$

For example, one of the 729 estimates was calculated using the "upper" value for all $c h 4, u$, and $w$ measurements, the "lower" value for all $v$ and $t$ measurements, and the "measured" value for all $p$ measurements. So, for each period 729 estimates were performed using OTM calculations and the different possible levels of each variable. This produced an average and standard deviation of estimates for each period as a percentage of the actual "measured" estimate. The "measured" estimate was the estimate made when all of the variables used their "measured" level (i.e., the value that would actually be reported).

From the 804 valid release periods a random sample of 100 periods were selected. All 729 combinations of variable levels were used to estimate mass emissions rates from these 100 periods. Measurement uncertainty as a percentage of the actual estimate was inferred from these 100 periods. The percentage of the estimate was scaled around the measured estimate which had a value of one. The measurement uncertainty as percentage of the estimate was determined using the following methodology.

1. The percentage of the estimate was calculated for each of the 729 results for each period.

2. From these 729 results a single estimate was randomly selected to represent the given period resulting in 100 samples.

3. The mean, standard deviation ( $\sigma$ ), and standard error (SE) of these periods were calculated. The SE was calculated using Equation 24, where $n$ represents the number of samples (100).

4. The measurement uncertainty of the 100 samples was calculated as $[ \pm 1.96 * \mathrm{SE}]$ representing the 95\% CL of the standard normal distribution.

5. This process was repeated for 1000 iterations and the average measurement uncertainty was used as the final measurement uncertainty for all periods.

$$
S E=\frac{\sigma}{\sqrt{n}}
$$

Equation 24

An example a single iteration consisting of 100 samples is presented in Figure 31. The 1000 iterations of measurement uncertainty as a percentage of the estimate are presented in Figure 32. The final measurement uncertainty of OTM estimates due to instrumentation alone was determined to be $\pm 3.8 \%$ of the "measured" value. 


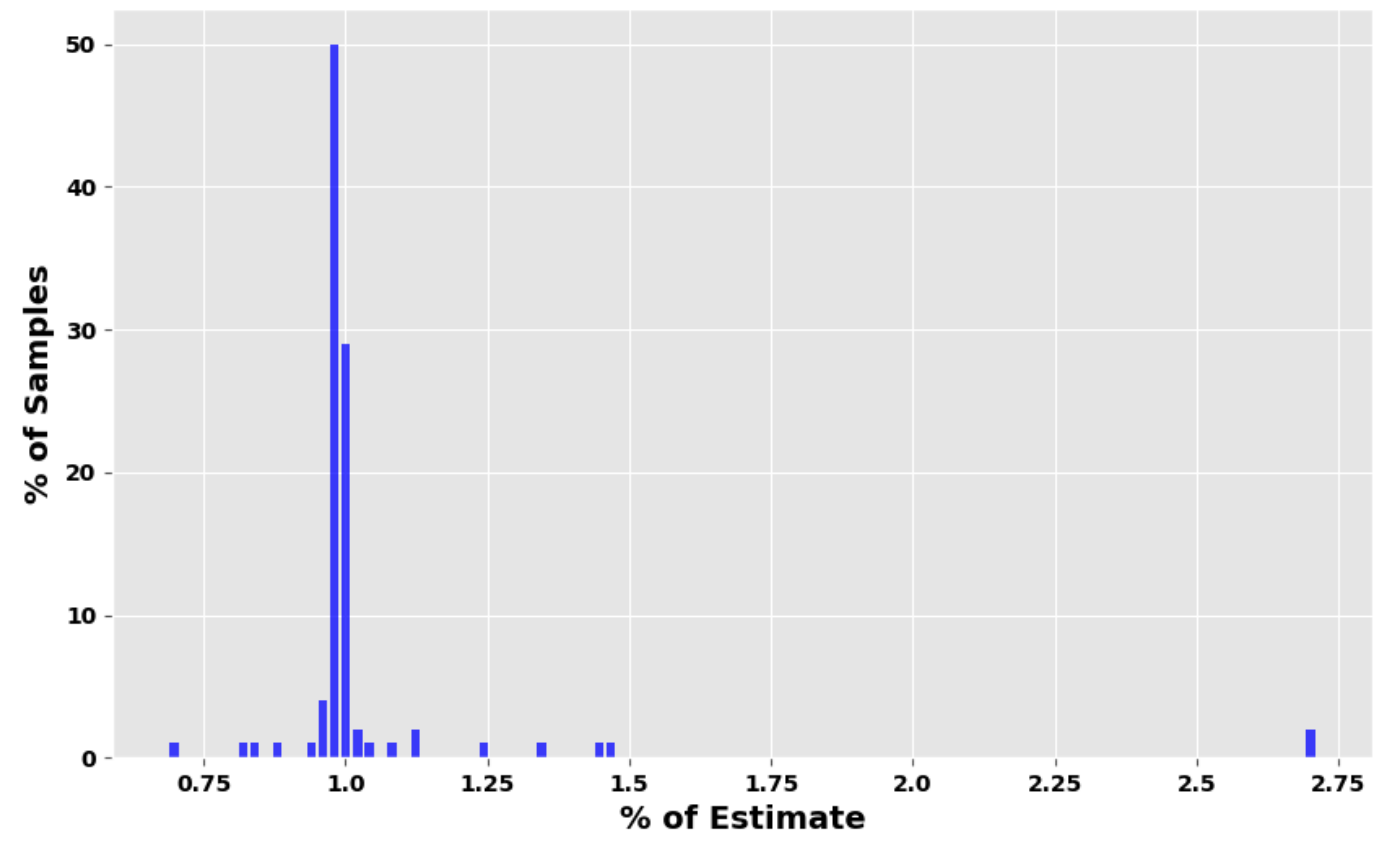

Figure 31. OTM measurement uncertainty distribution.

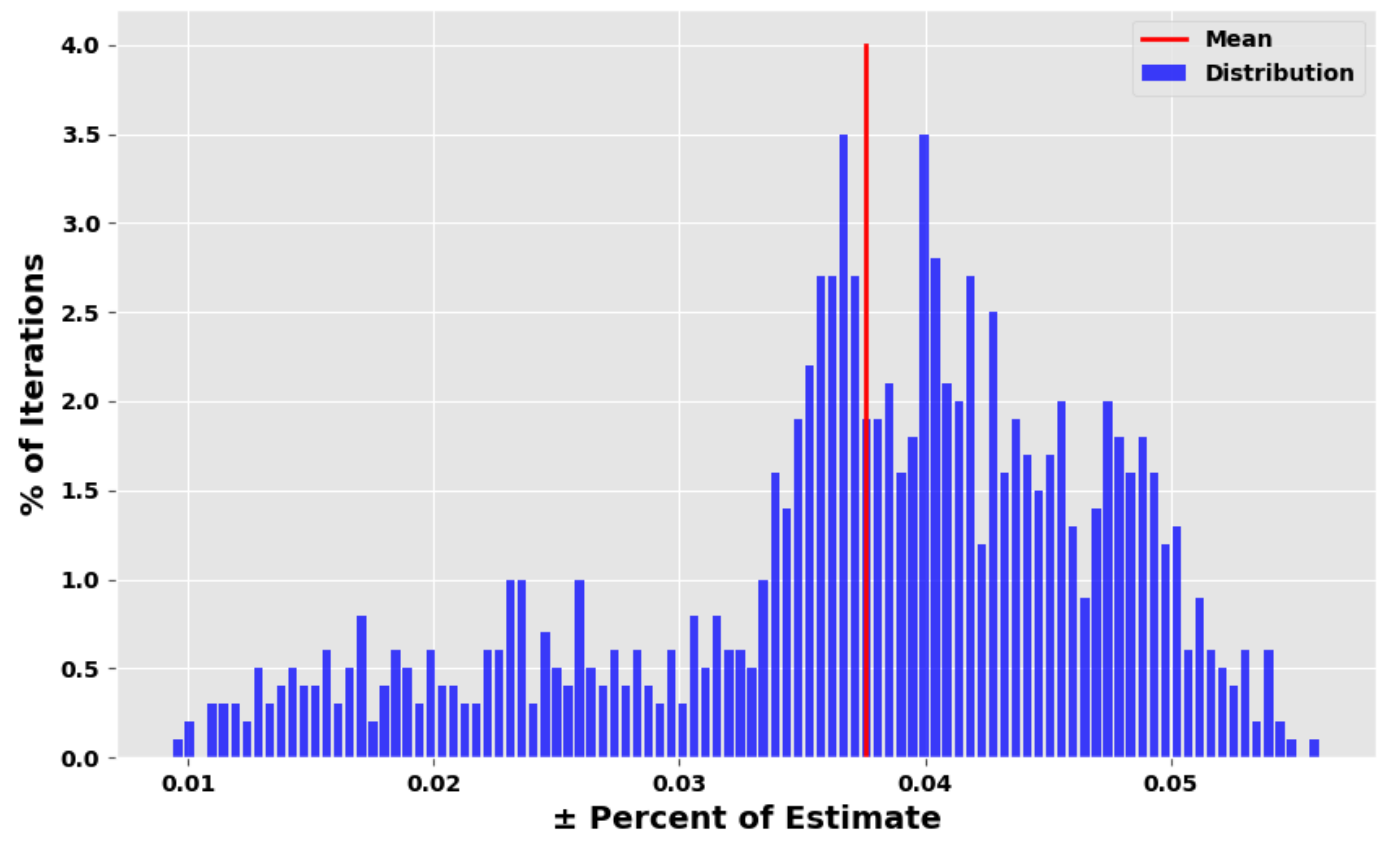

Figure 32. Bootstrapped OTM measurement uncertainty as a percentage of the estimate.

\subsubsection{EC Uncertainty}

EC uncertainty was quantified in two ways. The first was a random uncertainty estimation based on the method proposed by F\&S [83]. This method attempts to quantify the sampling error of EC measurements by calculating the square root of the "variance of the covariance". To quantify this error, the method includes terms for the auto- and cross-covariance of atmospheric fluxes. EddyPro ${ }^{\circledR}$ was able to calculate 
this uncertainty and denoted it as "rand_err_ch4_flux" in the output file and had units of $\left[\mu \mathrm{mol} / \mathrm{m}^{2} \mathrm{~s}\right]$. This random uncertainty is the "absolute uncertainty of the flux" $\left(\sigma_{\mathrm{F}}\right)$ which has the same units as the flux in question [144]. The random uncertainty estimate can be divided by the flux to give a normalized Fractional Flux Sampling Error (FFSE). F\&S concluded that this value was typically around $25-30 \%$ for trace gas fluxes. One fallback of the F\&S method is that it does not included any uncertainty associated with footprint heterogeneity. Equation 25 was used by EddyPro® to determine $\sigma_{\mathrm{F}}$ for an individual period.

$$
\sigma_{F}=\frac{1}{\sqrt{n}}\left(\sum_{p=-m}^{m} \gamma_{w, w}(p) \gamma_{c, c}(p)+\sum_{p=-m}^{m}(p) \gamma_{c, w}(p)\right)^{1 / 2}
$$

Equation 25

In Equation 25, $\gamma_{w, w}$ is the variance of $w$ estimated from the data, $\gamma_{c, c}$ is the variance of methane estimated from the data, $\gamma_{c, w}$ is the estimated covariance of the vertical wind speed and methane, $n$ is the number of samples in the period $(9,000$ for 15 -minute periods at $10 \mathrm{~Hz})$, and $m$ is a number of samples sufficiently large to capture the integral time scale (also calculated by EddyPro ${ }^{\circledR}$ ). The F\&S FFSE was scaled like the OTM measurement uncertainty since it was reported as a percentage of the estimate of the period.

Of the 804 periods with valid methane flux measurements and a controlled release present there were 776 periods that also produced a valid random error estimate for methane flux. To determine the average FFSE, a bootstrapping method was utilized. One thousand iterations were executed in which 776 periods were sampled with replacement. For each of the 1000 iterations the mean, standard deviation and standard error were calculated in the same way that the OTM measurement uncertainty. For the 1000 iterations the mean FFSE was $34.7 \%$. This uncertainty was about $5 \%$ higher than the expected value reported by F\&S for trace gas fluxes. However, the results are not unexpected given the lack of homogeneity inherent in a point emission source. The results of the 1000 iterations are presented in Figure 33.

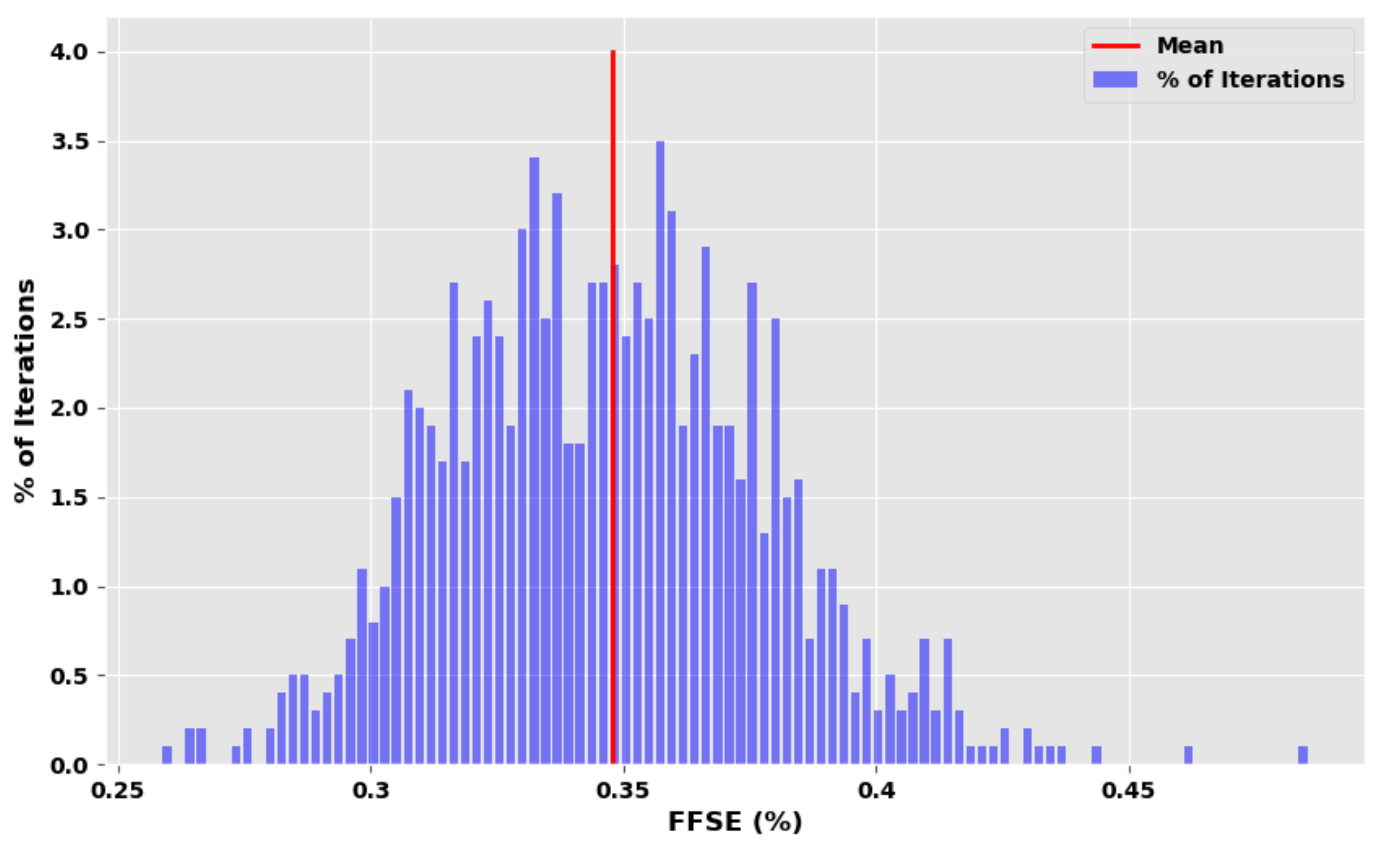

Figure 33. Bootstrapped mean FFSE of 776 periods.

A second method used for uncertainty estimation of EC fluxes is that of Hollinger and Richardson (2005) (H\&R) [82]. Their research presented two options for quantifying flux uncertainty, one with multiple towers and one using the same tower measuring at different times. The option involving only one tower utilized 
data from the same tower measured exactly 24-hrs apart. The reason for the 24-hr spacing was to reduce diurnal variation in the estimates. To ensure that the periods of comparison were sufficiently similar, several qualifiers were utilized to govern accepted period pairs. Their original research was focused on differences in heat, water vapor, and $\mathrm{CO}_{2}$ fluxes. The qualifiers for acceptable periods were based on the drivers of these types of fluxes. The qualifiers for similar periods were defined as follows:

- Photosynthetic Photon Flux Density (PPFD) Difference less than $75 \mu \mathrm{mol} / \mathrm{m}^{2} \mathrm{~s}$

- $\quad$ Air Temperature Difference less than $3^{\circ} \mathrm{C}$

- Vapor Pressure Deficit Difference less than $200 \mathrm{~Pa}(0.2 \mathrm{kPa})$

- Wind Speed Difference less than $1 \mathrm{~m} / \mathrm{s}$

When two periods separated by 24-hrs met all defined criteria they were accepted for use in the calculation to determine the standard deviation of the measurement error $[\sigma(\delta q)]$ as defined by H\&R. The standard deviation of the measurement uncertainty can be calculated from the accepted 24-hr periods with Equation 26.

$$
\sigma(\delta q)=\frac{\sigma\left(x_{1, t}-x_{2, t}\right)}{\sqrt{2}}
$$

Equation 26

Where $\sigma(\delta q)$ is the standard deviation of the measurement uncertainty, $x_{1, t}$ and $x_{2, t}$ are flux measurements taken 24-hrs apart which meet the defined criteria and $\sigma\left(x_{1, t}-x_{2, t}\right)$ is the standard deviation of the differences of those respective pairs. The derivation of $\sigma(\delta q)$ follows the logic that two with measured fluxes of $X_{1}$ and $X_{2}$ have measurement uncertainties associated with them of $\delta q_{1}$ and $\delta q_{2}$. Because the expected difference between $X_{1}$ and $X_{2}$ of sufficiently similar periods would be zero, it follows that the variance of $\left(\delta q_{1}-\delta q_{2}\right)$ is equal to the variance of $\left(X_{1}-X_{2}\right)$. Hence the standard deviation of the uncertainty can be inferred from the standard deviation of the measurements whose expected values are equivalent [82].

To quantify the uncertainty of the flux measurements from the MECT at the farm, background periods were used since they were expected to have the same value 24-hrs apart. Periods with controlled releases of the same rate and distance spaced 24-hrs apart were rare and variations in wind direction would greatly impact the estimations of these periods. The metrics of interest in this research were the natural methane flux from the soil and the "background" OTM estimation. Yamulki et al, determined that the primary drivers of methane flux from soils in which grazing animals were sometimes active were ambient temperature and rainfall [145]. To determine the natural difference in methane flux, the qualifiers were defined as follows:

- No precipitation between periods

- $\quad$ Air Temperature Difference less than $3^{\circ} \mathrm{C}$

OTM relies on stability class assumptions and is a direct product of the wind speed, in addition to the natural drivers of methane flux. For OTM periods to be accepted they were required to meet the following criteria:

- No precipitation between periods

- $\quad$ Air Temperature Difference less than $3^{\circ} \mathrm{C}$

- $\quad$ Photosynthetic Photon Flux Density (PPFD) Difference less than $75 \mu \mathrm{mol} / \mathrm{m}^{2} \mathrm{~s}$

- Wind Speed Difference less than $1 \mathrm{~m} / \mathrm{s}$

PPFD $\left[\mu \mathrm{mol} / \mathrm{m}^{2} \mathrm{~s}\right]$ is a measure of light intensity approximately equal to $4.6 \mathrm{~W} / \mathrm{m}^{2}$ [146]. In this research the variable of solar loading in $\mathrm{W} / \mathrm{m}^{2}$ was recorded by a pyranometer and converted to PPFD. From the background periods collected in between controlled releases there were 1208 periods that contained both valid OTM and EC estimates. From these 1208 periods there were 107 accepted pairs of periods that met the criteria defined for EC and 65 pairs of periods that met the criteria for OTM. The 107 accepted EC pairs had an average difference in flux measurements of $-0.0006 \mu \mathrm{mol} / \mathrm{m}^{2} \mathrm{~s}$. The mean flux and mean absolute flux of the 214 periods in the 107 pairs were -0.0076 and $0.089 \mu \mathrm{mol} / \mathrm{m}^{2} \mathrm{~s}$, respectively. The standard 
deviation of the measurement differences, $\sigma\left(X_{1}-X_{2}\right)$, was $0.287 \mu \mathrm{mol} / \mathrm{m}^{2} \mathrm{~s}$. The standard deviation of the measurement uncertainty, $\sigma(\delta q)$, was $0.203 \mu \mathrm{mol} / \mathrm{m}^{2} \mathrm{~s}$. The flux differences of the 107 periods are presented in Figure 34. There were six flux differences that had an absolute difference greater than 1 $\mu \mathrm{mol} / \mathrm{m}^{2} \mathrm{~s}$, which are not present in Figure 34 .

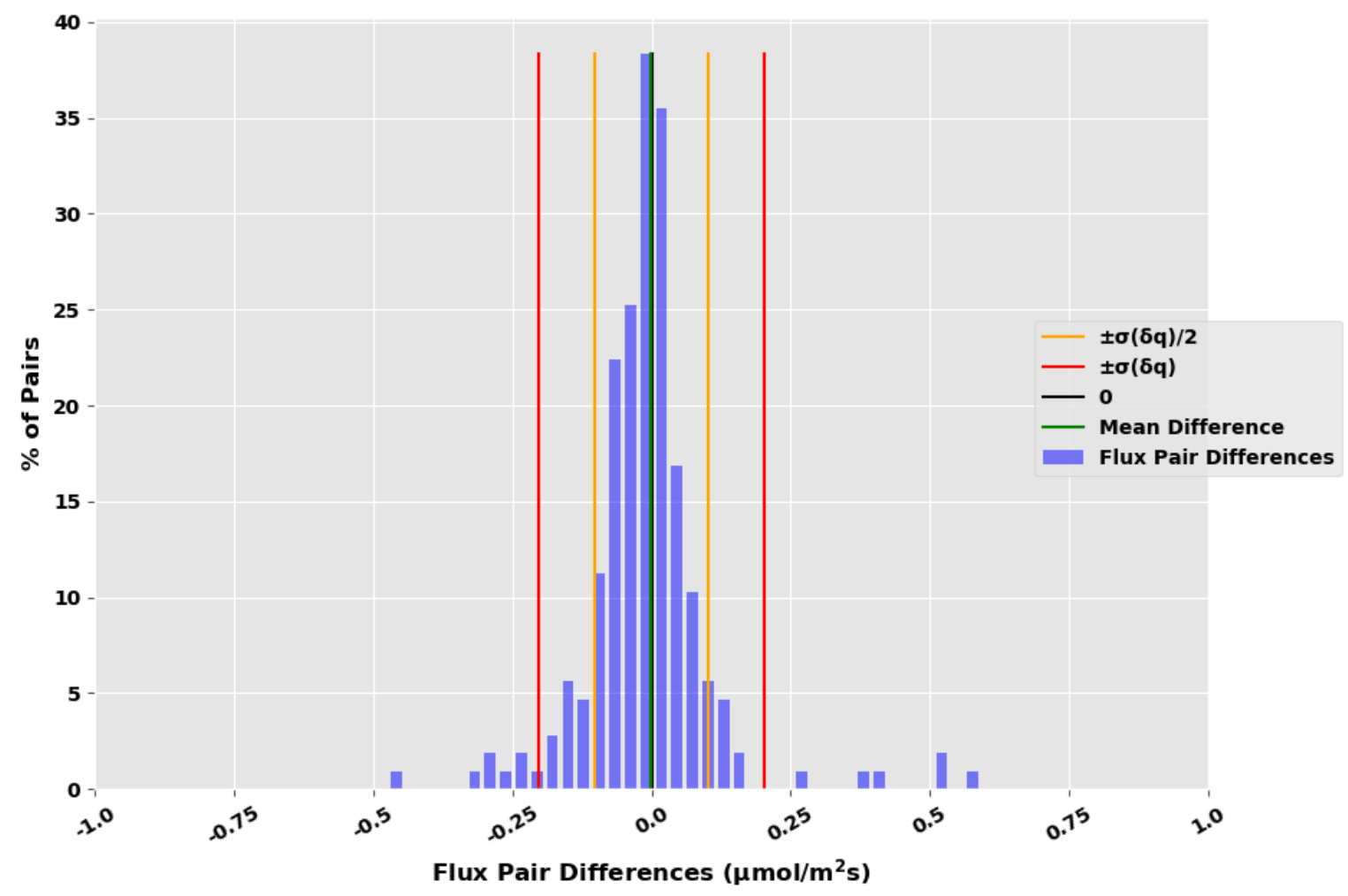

Figure 34. Distribution of flux pair differences used for EC H\&R uncertainty.

The goal was to determine the uncertainty from this method as a percentage of the flux, so that it could be compared with the other methods previous discussed. To determine the uncertainty as a percentage of the estimate a bootstrap of 1000 iterations on the 107 pairs was performed. For each of the iterations a sample size of 107 pairs was selected with replacement. The absolute mean of the flux values, the mean difference of the pairs, the standard deviation of measurement uncertainty, and the SE of the measurement uncertainty were calculated for each iteration. The SE as presented in Equation 24. From 1000 iterations the mean SE was $0.02 \mu \mathrm{mol} / \mathrm{m}^{2} \mathrm{~s}$ and the mean absolute flux was $0.89 \mu \mathrm{mol} / \mathrm{m}^{2} \mathrm{~s}$. I again used the assumption that $[ \pm 1.96$ * SE] was the $95 \%$ CL of the uncertainty, as was performed for the OTM uncertainty. The average $95 \%$ CL was $0.037 \mu \mathrm{mol} / \mathrm{m}^{2} \mathrm{~s}$, which, as a percentage of the absolute flux, was on average $42 \%$ of the mean absolute flux. The mean flux differences of the 1000 iterations of 107 pairs and the mean CL of the iterations are presented in Figure 35. 


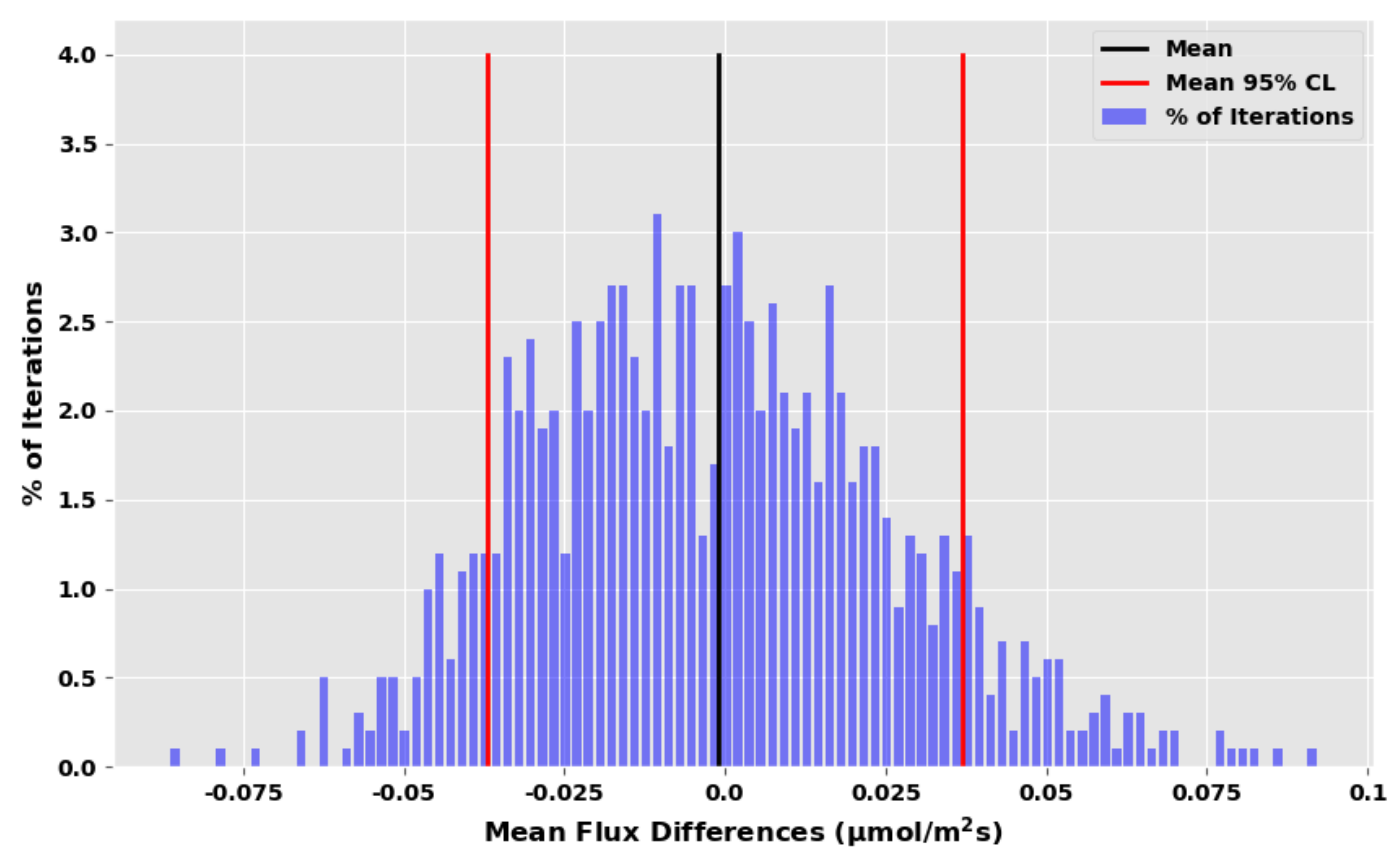

Figure 35. Mean EC flux pair differences of 1000 iterations.

The method was repeated to determine the uncertainty in OTM measurements since the differences between periods with no emissions also had an expected difference of zero. To account for the distance variable in the OTM calculations, the OTM estimates for each period used by the 24-hour pairs were calculated for each of the different controlled release distances. This resulted in an increasing uncertainty with distance. However, the average magnitude of the OTM estimate also increased with distance. This resulted in a negligible change in the uncertainty as a percentage of the estimate. The mean results of the 1000 iterations are presented in Table 51.

Table 51. Results of H\&R method applied to OTM measurements.

\begin{tabular}{|c|c|c|c|c|c|}
\hline $\begin{array}{c}\text { Distance (m) } \\
\text { for Calculation }\end{array}$ & $\boldsymbol{\sigma}\left(\mathbf{X}_{\mathbf{1}}-\mathbf{X}_{\mathbf{2}}\right)$ & $\boldsymbol{\sigma}(\mathbf{\delta q})$ & $\mathbf{9 5 \%} \mathbf{C I}$ & $\begin{array}{c}\text { Mean Estimate } \\
\text { of Periods }\end{array}$ & $\begin{array}{c}\mathbf{9 5 \%} \mathbf{C I} / \\
\text { Mean Estimate }\end{array}$ \\
\cline { 2 - 6 }$[\mathbf{g} / \mathbf{s}]$ & {$[\mathbf{g} / \mathbf{s}]$} & {$[\mathbf{g} / \mathbf{s}]$} & {$[\mathbf{g} / \mathbf{s}]$} & 0.007 & $17 \%$ \\
\hline 42 & 0.007 & 0.005 & 0.001 & 0.012 & $17 \%$ \\
\hline 57 & 0.012 & 0.008 & 0.002 & 0.018 & $17 \%$ \\
\hline 72 & 0.018 & 0.012 & 0.003 & 0.045 & $17 \%$ \\
\hline 119 & 0.045 & 0.032 & 0.008 & \multicolumn{4}{|c}{} \\
\hline
\end{tabular}

Rannik et al. (2016) utilized several methods of flux uncertainty estimation, including that proposed by F\&S found random uncertainties of the error estimates to be on the order of 10-30\% [147]. Deventer et al. (2019) measure methane fluxes with an LI-7700 and utilized the H\&R 24-hr method. They reported uncertainties of 30-minute periods were typically between $6-41 \%$ [87].

\subsection{Summary of Traditional Methods}

Two traditional methods were utilized to indirectly quantify methane mass emission rates from controlled release experiments. These methods have been used independently in previous research; however, they have not been tested against each other using concurrent measurements. The EC footprint models produced 
a limited number of periods $(\mathrm{n}=142)$ with valid results during controlled release tests. The EC footprint and OTM results were compared across these 142 periods. It is worth noting that nearly all the accepted periods $(n=140)$ occurred at the shortest release rate distance of $42 \mathrm{~m}$. This finding suggests that a taller tower would be required under similar micrometeorological conditions if the potential sources were more than $50 \mathrm{~m}$ from the tower position. However, if the MECT were positioned somewhere on the site, as opposed to an adjacent roadway the distance to components could be reduced. The mobility of the system would allow the user to position in such a way that would optimize distances to potential sources. A comparison of the results from these periods are presented in Figure 36. The predictions from the Taguchi OTM resulted in an RMSE that was $33.8 \%$ and $27.5 \%$ less than the FFP and KM estimates, respectively. All three methods tended to underpredict across all release rates.

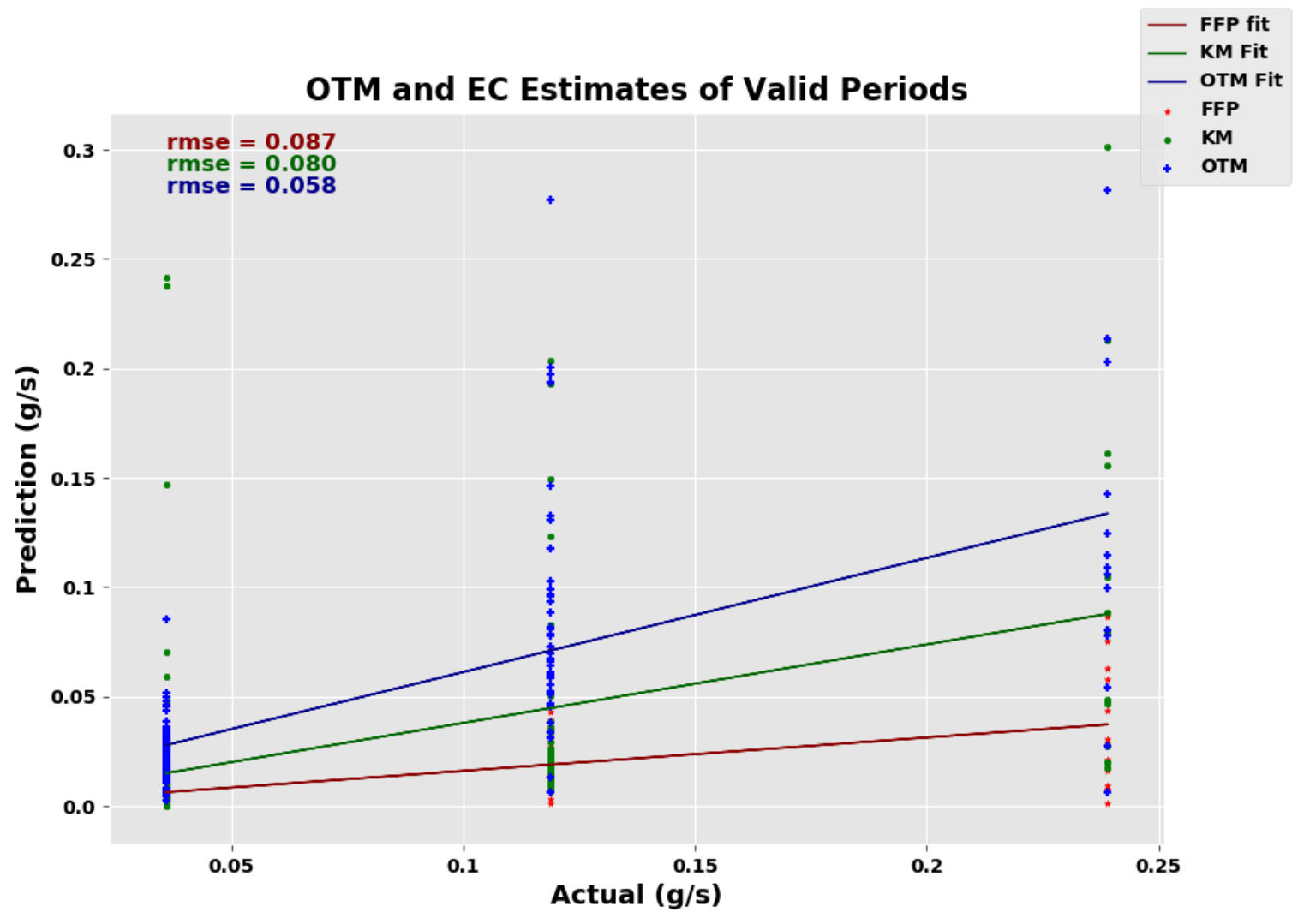

Figure 36. Comparison of traditional method results.

Attempts have been made to understand the uncertainty of these methods, particularly EC, however the uncertainties of these methods vary widely and are dependent upon specific conditions during data collection. Multiple methods of quantifying uncertainty have been presented for both methods. The OTM method measurement uncertainty due to instrument error was determined from a bootstrapped sample of 100 random periods. In addition to the measurement uncertainty due to devices, the uncertainty due to the variability of estimates during similar micrometeorological conditions was quantified via the H\&R method traditionally used for EC uncertainty [82]. The uncertainty of the EC measurements was also quantified utilizing two different techniques. The F\&S method results were produced from EddyPro ${ }^{\circledR}$ calculations as the methane flux random error [83]. This method does not consider footprint variability, so it was smaller than that of the second method developed by H\&R. This method is meant to account for the variability in measurements caused by measurement and micrometeorological differences. This method was used on both OTM and EC periods which measured the "background" during the controlled release experiments. During 
the background periods, no controlled release was present. The resultant uncertainty therefore was the uncertainty in the measurement method to do lack of repeatability. The uncertainty methods and results are summarized in Table 52. In terms of combined uncertainty, it would be relevant to combine the uncertainties of the OTM measurements since they present two different forms of uncertainty. The difference between the F\&S and the H\&R methods is a result of the inclusion of the uncertainty in footprint heterogeneity in H\&R. Based on the uncertainty estimates, this additional uncertainty could add about $7 \%$ uncertainty to EC measurements. For a final uncertainty estimation of OTM measurements the combined uncertainties of the instrument error and measurement variance of $H \& R$ were used. However, the instrument error had a minor affect only increasing the estimated measurement uncertainty to $\pm 17.4 \%$ by using the sum of the squares presented in Equation 23. For EC, the larger H\&R uncertainty ( $\pm 42 \%$ of flux) was utilized because the inclusion of uncertainty related to footprint was considered critical for point source quantification.

There were 804 periods that produced valid OTM estimates. The RMSE of the OTM estimates was 0.123 $\mathrm{g} / \mathrm{s}$. The average percent error of these periods was $73.8 \%$. Previous studies have utilized this value as the uncertainty of the method when discussing the uncertainty associated with the OTM method [53].

Table 52. Summary of uncertainty quantification methods.

\begin{tabular}{|c|c|l|c|}
\hline $\begin{array}{c}\text { Quantification } \\
\text { Method }\end{array}$ & $\begin{array}{c}\text { Uncertainty } \\
\text { Method }\end{array}$ & \multicolumn{1}{|c|}{ How it is presented } & Result \\
\hline OTM & $\begin{array}{c}\text { Instrument } \\
\text { Measurement } \\
\text { Uncertainty }\end{array}$ & $\begin{array}{l}\text { The range of uncertainty as a percentage of the OTM } \\
\text { estimate for any period based on the uncertainty of the } \\
\text { instruments used to record data. }\end{array}$ & $\pm 3.8 \%$ \\
\hline EC & $\begin{array}{c}\text { F\&S Random Flux } \\
\text { Error }\end{array}$ & $\begin{array}{l}\text { The range of the Normalized FFSE (\%) of the EC flux } \\
\text { estimate for any period based on the variance of the } \\
\text { covariance of the period. }\end{array}$ & $\pm 35 \%$ \\
\hline EC & $\begin{array}{c}\text { H\&R 24-hr } \\
\text { Difference Method }\end{array}$ & $\begin{array}{l}\text { The range of measurement uncertainty of the method } \\
\text { due to randomness in the measurement. }\end{array}$ & $\pm 42 \%$ \\
\hline OTM & $\begin{array}{c}\text { H\&R 24-hr } \\
\text { Difference Method }\end{array}$ & $\begin{array}{l}\text { The range of measurement uncertainty of the method } \\
\text { due to randomness in the measurement. }\end{array}$ & $\pm 17 \%$ \\
\hline
\end{tabular}

\subsection{Combined Method Modeling}

One of the goals of this research was to develop methods that produce more accurate results or reduce the uncertainty of traditional estimates. The results of the traditional methods were compared to concurrent controlled releases and different methods of estimating uncertainty were evaluated for each method. Here an attempt to better predict emissions rates utilizing data from the two methods in combination with machine learning was explored.

\subsubsection{Dataset Formation}

The outputs from OTM and EddyPro ${ }^{\circledR}$ calculations were used to form the primary datasets for combined evaluations. The 15-minute periods included were those that produced valid estimates of mass emission rates from OTM and valid estimates of methane flux from EddyPro ${ }^{\circledR}$. As previously discussed, there were 804 valid controlled release periods and 1208 valid background periods. To balance the dataset and not give more weight to background periods only 804 randomly selected background periods were used in model evaluation. This resulted in a full dataset of 1608 periods. The variables used for analysis were from both default processing methods. The OTM outputs were selected based on those relevant to the method 
and other available data averages for the period. The number of valid output variables from the OTM calculations was 27. The number of EddyPro ${ }^{\circledR}$ variables in the full output file based on the settings used was 182 . From this list all non-number or identifying variables were removed resulting in 146 variables. The distance and StS direction were then added to the set of variables. The total number of possible variables in the dataset was 175 . Variables that were used as inputs to the models were defined as "features". There pool of potential features consisted of the 175 variables. Using all features produced a high ratio (greater than 10\%) of features to periods (1608). It was expected that not all features would have a significant impact on model results. Therefore, methods for reducing the size of the feature set were explored.

To reduce the number of features and create alternative datasets two measures were taken. For the first alternative dataset, all features were removed that did not have a Pearson Correlation p-value less than 0.05 when compared to the controlled release rate. The magnitude of the Pearson correlation was not considered, only the associated p-value which was found as described by Equation 21. This eliminated all features that showed no correlation with the controlled release rate. This release rate correlated (RRC) dataset contained only 54 features. A second alternative dataset further reduced the number of features by eliminating those that were highly correlated with one another. High correlation reduction was performed by grouping all features which had cross-correlation coefficients greater than 0.75 . The two major variable groups were those associated with temperature or atmospheric water vapor, and those associated with methane. There were 32 highly correlated features that were eliminated by this filter. The complete list of correlated features is available in Appendix A. This third dataset contained only RRC features that were not eliminated by the high-correlation filter (HCF). The total number of features in the RRC-HCF dataset was 22 . The three datasets used in the initial machine learning model evaluations are listed below with their respective number of features.

- $\quad$ Both OTM and EC with All Features (175)

- $\quad$ Both OTM and EC with RRC Features (54)

- $\quad$ Both OTM and EC with RRC-HCF Features (22)

\subsubsection{Model Selection}

Several machine learning techniques were considered for use on the combined datasets. There are nearly infinite combinations of features, preprocessing, and machine learning algorithms now in use by data scientists and engineers. Choosing the best algorithms for novel applications is therefore not a simple task. To assist in narrowing the breadth of these options, several items were considered based on the nature of the problem and desired solution.

1. The test/train dataset was limited. An objective of future research would be to extend the available data to more sites and release scenarios and in turn enhance any modeling methodologies. However, for the purpose of this research there was a limited dataset consisting of the controlled releases performed at the farm.

2. Many machine learning algorithms can be improved with data scaling or normalization as a preprocessing step. However, if the method developed was to be applicable to new sites and scenarios the scaling or normalization of features would require adjustment with each new scenario. For example: if the dataset that the model was trained on did not contain a wider range of values than those measured at a novel site retraining with a different normalization or scaling technique may be necessary to maintain accurate results. As a result, preference was given to applications that were able to perform well on input data that did not require scaling or normalization.

3. Better interpretability was desired when it could be achieved. This is another drawback of datascaling. It makes inputs much more difficult to interpret without extensive knowledge of the dataset and its makeup. Models can also affect interpretability. For example, deep NNs often act as a black box. 
None of these criteria were used to eliminate modeling options but were considered during selection and filtering. Several online resources were utilized to select the initial machine learning methods to test. Microsoft Azure Cloud Computing Services, Python's open source scikit-learn (sklearn), and SAS Analytics, Artificial Intelligence and Data Management Software all provide decision diagrams on how to select the best machine learning method for a given application [148]-[150]. The flow of each of these diagrams is presented in Figure 37. 


\section{SAS Software}

Dimension

Reduction?

No

\section{Have Responses?}

Yes

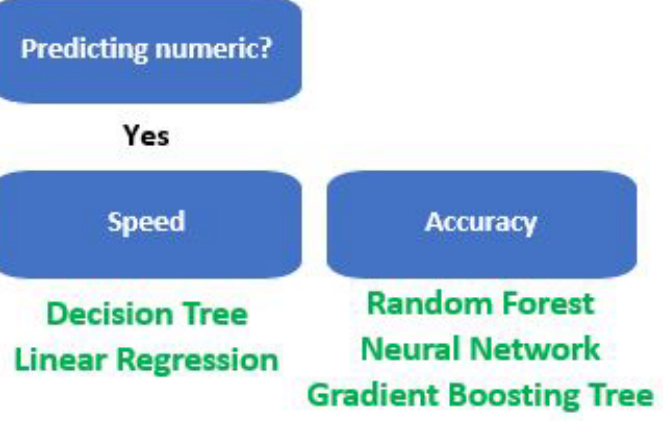

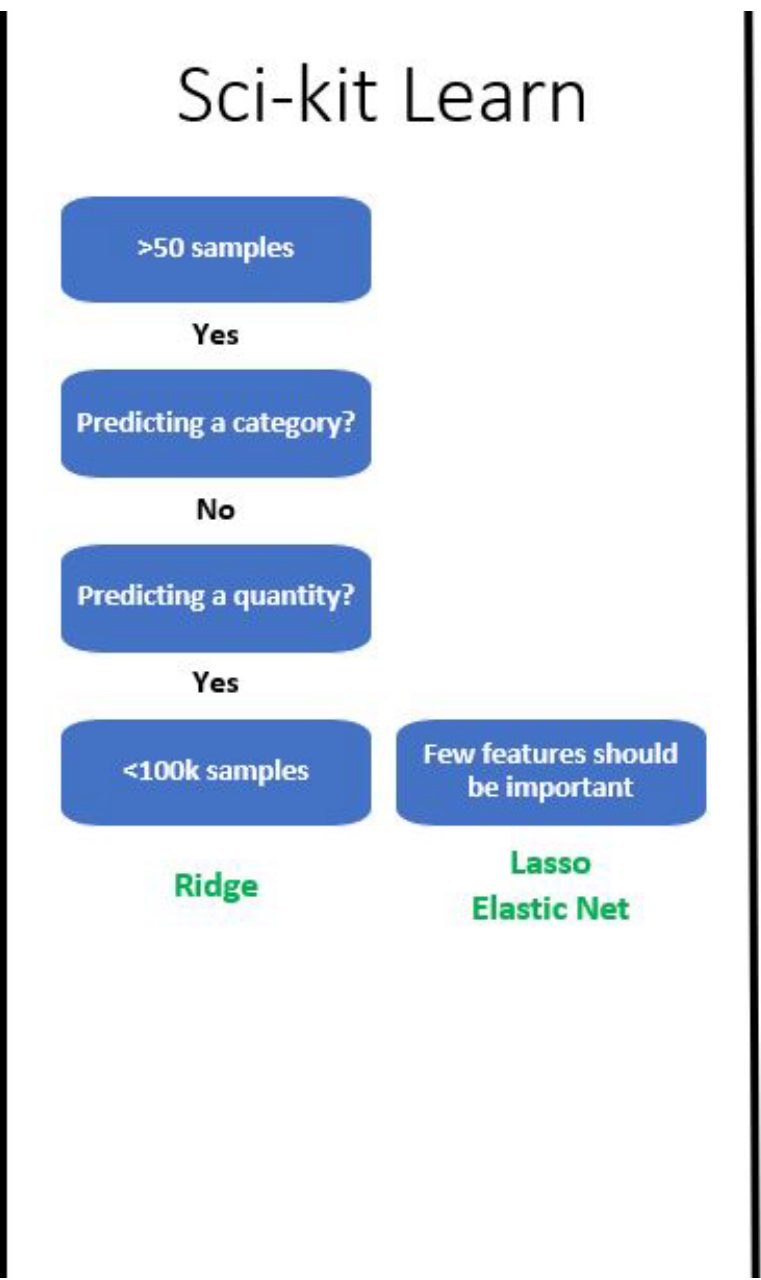

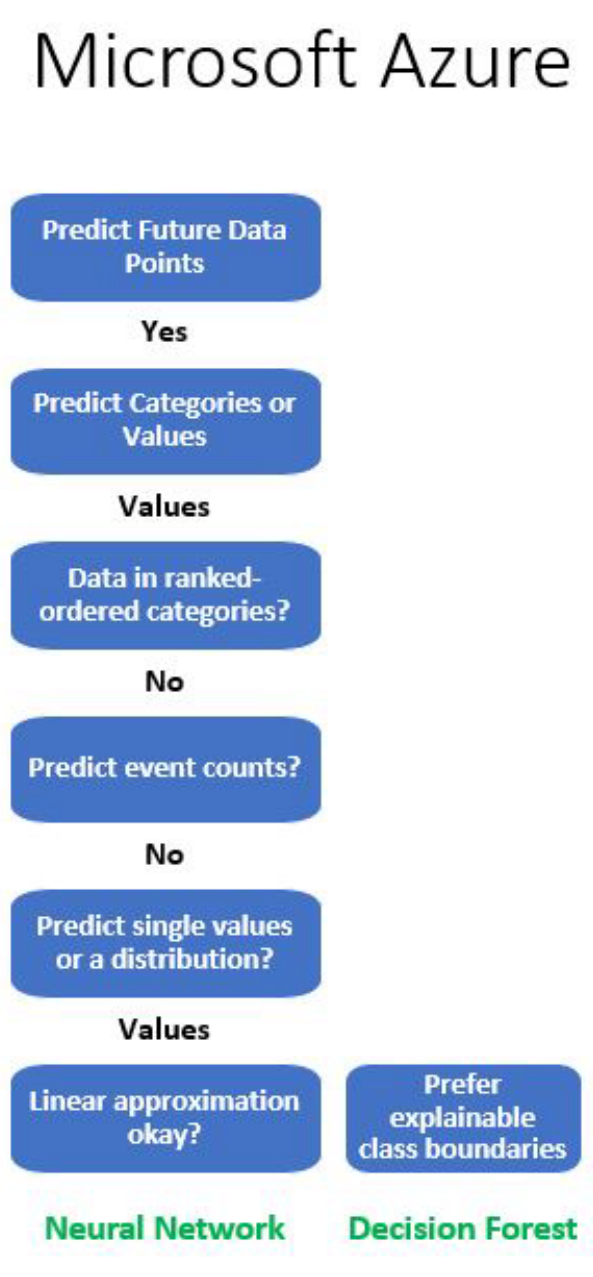

Figure 37. Selection of machine learning algorithms [148]-[150]. 
Using these suggestions, the list of machine learning algorithms to test was narrowed to the following:

- $\quad$ Linear Regression Techniques

$$
\begin{array}{ll}
\circ & \text { Linear } \\
\circ & \text { Ridge } \\
\circ & \text { Lasso } \\
\circ & \text { Elastic Net }
\end{array}
$$

- Decision Trees and Forests
○ Random Forest Regression
- Decision Tree Regression

- $\quad$ Neural Networks

The sklearn package was utilized to implement the initial modeling techniques [148]. The Python package contains functions and classes for well-known regression and classification techniques. The following regression methods from sklearn were used in initial model evaluation.

- ensemble.RandomForestRegressor (RF)

- tree.DecisionTreeRegressor

- linear_model.LinearRegression

- linear model.Ridge

- linear_model.Lasso

- linear_model.ElasticNet

- $\quad$ neural_network.MLPRegressor (NN)

\subsubsection{Initial Model Evaluation}

Each of the models were evaluated using the different datasets previously described. Models were trained on $75 \%$ of the periods from the controlled release experiments and tested on the remaining $25 \%$. Models were evaluated and compared using the RMSE, presented in Equation 19, of the test periods. The matrix of models and datasets is presented in Table 53, with their respective resultant test RMSE results. The results were used to narrow the focus of model selection. RF and NN acronyms are used to describe the specific models utilized, not the general methods, which will be spelled out.

Table 53. Initial machine learning results (RMSE [g/s]).

\begin{tabular}{|c|c|c|c|c|c|c|c|c|}
\hline Scaled & $\begin{array}{c}\text { Variable } \\
\text { Set }\end{array}$ & RF & $\begin{array}{c}\text { Decision } \\
\text { Tree }\end{array}$ & $\begin{array}{c}\text { Linear } \\
\text { Regressor }\end{array}$ & $\begin{array}{c}\text { Ridge } \\
\text { Regressor }\end{array}$ & $\begin{array}{c}\text { Lasso } \\
\text { Regressor }\end{array}$ & $\begin{array}{c}\text { Elastic- } \\
\text { Net } \\
\text { Regressor }\end{array}$ & NN \\
\hline No & All & 0.0424 & 0.0445 & 4.1866 & 1.3740 & 0.0648 & 0.0643 & 275082 \\
\hline No & RRC & 0.0446 & 0.0613 & 0.0555 & 0.0572 & 0.0648 & 0.0647 & 7680 \\
\hline No & RRC-HCF & 0.0453 & 0.0599 & 0.0556 & 0.0563 & 0.0660 & 0.0660 & 0.994 \\
\hline Yes & All & 0.0409 & 0.0536 & 4.0802 & 0.0583 & 0.0660 & 0.0660 & 0.426 \\
\hline Yes & RRC & 0.0448 & 0.0627 & 0.0555 & 0.0561 & 0.0660 & 0.0660 & 0.113 \\
\hline Yes & RRC-HCF & 0.0453 & 0.0577 & 0.0556 & 0.0554 & 0.0660 & 0.0660 & 0.085 \\
\hline
\end{tabular}

From these results several conclusions were made to narrow the modeling options. While one could simply take the best dataset and model combination as final, based on the engineering problem at hand several preferences were given to specific results. Unscaled datasets were considered more valuable than scaled datasets. This preference was based on the reality that if the method were to be used at new sites it would be difficult to apply the same scaling used in training the model to novel data. The alternative would be that 
as more data was collected scaling would become more robust and as models received more training on novel data they would improve. Preference was also given to datasets with fewer features. This was to make models more interpretable. It also avoids giving unnecessary weight to features with little or no significance to the problem at hand. Finally, for the purpose of comparison it was desirable to use the same feature set on the selected regressors.

Before narrowing the modeling options, models were divided into three categories:

$$
\begin{aligned}
& \text { - "Trees and Forests" - the RF and Decision Tree regressors } \\
& \text { - "Linear Regressors" - the Linear, Ridge, Lasso and Elastic-Net regressors } \\
& \text { - "Neural Networks" - the default Multi-Layer Perceptron regressor }
\end{aligned}
$$

The best performing models from each of these categories would be further evaluated. The top performers in each category are listed below.

- $\quad$ Trees and Forests: RF regressor using all scaled features.

- $\quad$ Linear Regressors: Linear Regressor using RRC unscaled features.

- Neural Network: RRC-HCF scaled features.

Since none of the best performing models utilized the same feature sets, other considerations were used to select the desired set. As previously stated, datasets containing fewer features were desired for both interpretability and simplicity. For these reasons, the RRC-HCF was the most desirable feature set. This was the best performing feature set of the NN. The next best performing dataset on the NN was the RRC which resulted in a 33\% increase in the RMSE. For comparison, the RRC-HCF was the second-best feature set of the linear regressor and only caused a RMSE increase of $0.3 \%$ over the top feature set. Every feature set used in conjunction with the RF produced a lower RMSE then all but one other model (Decision Tree, all unscaled features). The difference between the best and worst performing RF evaluation was only $11 \%$. The best performing linear regressor and NN resulted in RMSEs that were $23 \%$ and $87 \%$ higher than the worst performing RF. These results suggested that neither scaling nor variable set had a major impact on RF performance. This was also an indication that the RF method required only a few critical features to produce more accurate results than the other models, as will be presented later. The NN had the largest difference between scaled and unscaled datasets. The NN was also the most affected by the size of the feature set. A reduction in the number of features by $69 \%$ and $87 \%$ improved the results of the NN by $74 \%$ and $80 \%$, respectively. These results suggested that the model was sensitive to the number of features used and performed better when more features not related to the problem were removed. These factors led to the selection of the RRC-HCF as the default dataset moving forward. For the sake of interpretability scaling was not implemented for the RF and linear regressor since it had minimal impact on results. However, without scaling the NN RMSE increased by 1 to 5 orders of magnitude depending on the feature set, therefore scaling the dataset was considered an essential preprocessing step when utilizing the NN. For the purpose of further model evaluation, the three model/feature set combinations used were:

1. RF with RRC-HCF unscaled features

2. Linear Regressor with RRC-HCF unscaled features

3. NN with RRC-HCF scaled features.

The initial RMSEs of these models with training on $75 \%$ of the controlled release dataset and testing on the remaining 25\% and all MSEEL audits are presented in Table 54. The periods compared to the MSEEL audits were those that occurred within \pm 1 day of the audit. These RMSEs were considered the model default values and were used for model improvement comparisons. 
Table 54. RMSE of machine learning algorithms.

\begin{tabular}{|c|c|c|c|}
\hline Model & $\begin{array}{c}\text { Controlled } \\
\text { Release } \\
\text { Training }\end{array}$ & $\begin{array}{c}\text { Controlled } \\
\text { Release } \\
\text { Testing }\end{array}$ & $\begin{array}{c}\text { MSEEL } \\
\text { Audits }\end{array}$ \\
\hline RF & 0.015 & 0.045 & 0.22 \\
\hline Linear Regressor & 0.050 & 0.056 & 2.05 \\
\hline NN & 0.066 & 0.085 & 0.49 \\
\hline
\end{tabular}

The linear regressor MSEEL audit RMSE was 4 and 9 times higher than the NN and RF, respectively. It also displayed a lack of robustness as its predictions were almost directly correlated to OTM estimates. It was also not sensitive to the removal of features that were correlated with one another. Basic linear regression models also cannot be tuned by adjusting model parameters, therefore the only way of improving such models is with more training data, which was not available. For these reasons it was removed from further consideration.

\subsubsection{Final Model Descriptions}

The RF algorithm builds upon several simpler regression tree models. A regression tree is a type of decision tree. Regression trees are an alternative to linear regression models when the relationships between features and targets are not linearly related. The regression tree works by splitting data based on randomly selected features within the dataset. Regression trees easily accommodate relationships between several features. For each set of randomly selected features, the regression tree splits the data based on the minimization of the residuals. The trees utilize the sum of squared residuals to determine the optimal split variable. This is performed for each randomly selected feature in the dataset. The value of the feature at which the sum of squared residuals is minimized results in a split in the decision tree. Once the data reaches a point that can no longer be split the tree is fully developed or "grown". The RF uses the results of many decision trees through a method known as "bagging". Bagging methods work well to reduce the variance of high-variance, low-bias procedures such as regression trees. When using bagging in combination with regression trees to form a RF, the same regression trees are fit many times with different bootstrapped samples of the training data. This means that trees grow differently depending on their random state. Trees can capture complex interactions between features and have relatively low bias when fully grown. Hastie et al. [151] describe the steps of a RF as follows:

1. For $b=1$ to $B$ :

a. Draw a bootstrap sample $Z^{*}$ of size $N$ from the training data.

b. Grow a RF tree $T_{b}$ from the bootstrapped data, recursively repeating the following steps for each terminal node of the tree, until minimum node size is reached.

i. Select $m$ features at random from the complete set of $p$ features.

ii. Pick the best feature split point from among the $m$ selected.

iii. Split the node into two daughter nodes.

2. Output the ensemble of trees $\left\{T_{b}\right\}_{1}^{B}$

3. To make a prediction about a new point $x$ :

$$
\text { a. } \hat{f}_{r f}^{B}(x)=\frac{1}{B} \sum_{b=1}^{B} T_{b}(x)
$$

As defined here $B$ is the number of trees in the forest. $T_{b}$ is an example of a tree in the forest, $p$ is the total number of available features in the set, and $m$ is a randomly selected subset of those features. RFs have the advantages of being a low-bias and relatively low-variance technique. They are less prone to over-fitting than individual decision trees, which often act as classifiers even for regression problems [151]. RFs also 
produce interpretable measures for determining the relative contribution of individual features. The importance of each feature at each split-point in the trees in the forest can be accumulated. In essence these "feature importance" values are a measure of each feature's impact on the model results because it is a function of how many split-points are most influenced by the feature in question.

Wager et al. recently developed a method for estimating the confidence interval for RFs [152]. Two methods used for determining the variance of a RF prediction are the Jackknife after bootstrap and the Infinitesimal Jackknife (IJ). Equation 27 and Equation 28 represent the variance calculation from these two methods, respectively. Using this notation, the RF estimator takes the form of Equation 29.

$$
\widehat{V}_{I J}^{\infty}=\sum_{i=1}^{n} \operatorname{Cov}_{*}\left[N_{i}^{*}, t^{*}(x)\right]^{2}
$$

Equation 27

Where $\operatorname{Cov}_{*}\left[N_{i}^{*}, t^{*}(x)\right]$ represents the covariance between $t^{*}(x)$ and the number of times $N_{i}^{*}$ the $i^{\text {th }}$ training example appears in the bootstrap sample.

$$
\widehat{V}_{J}^{\infty}=\frac{n-1}{n} \sum_{i=1}^{n}\left(\bar{t}^{*}{ }_{(-i)}(x)-\bar{t}^{*}(x)\right)^{2}
$$

Where $\bar{t}^{*}{ }_{(-i)}(x)$ is the average of $t^{*}(x)$ over all the bootstrap samples not containing the $i^{\text {th }}$ example and $\overline{t^{*}}(x)$ is the mean of all the $t^{*}(x)$.

$$
\hat{\theta}^{R F}(x)=\frac{1}{B} \sum_{b=1}^{B} t_{b}^{*}\left(x ; \xi_{b}, Z_{b 1}^{*}, \ldots, Z_{b n}^{*}\right)
$$

Where $Z_{b 1}^{*}$ to $Z_{b n}^{*}$ are the bagged samples of training data, $t_{b}^{*}(x)$ are the predicted values of from the $b^{\text {th }}$ tree of the forest where there are B trees in the forest. $\xi_{b}$ is the auxiliary noise of the system.

The uncertainty in a RF is the product of two types of noise: sampling (due to the randomness of data collection) and Monte Carlo (due to the finite number of bootstraps used for tree building). The estimates of the variance of a RF were based on the same set of bootstrapped replicates that were used to produce the predictions. In theory with an infinite number of bootstrapped samples the Monte Carlo error would be zero, since this is not possible, there will always be some Monte Carlo error due to the limited number of bootstraps available from a finite dataset. To compensate for this error in the prediction of the variance the combined results of the Jackknife after bootstrap and (IJ) were averaged which produce a more accurate representation of the variance. This was because the Jackknife after bootstrap variance tends to have an upward sampling bias and the IJ tended to have a downward sampling bias. To estimate the variance of the RFs used in this work, the functions developed by Polimis et al. were used. These functions are based on the R-code developed by Wager et al. for implementing the variance as calculated by their reduced-bias method [153].

ANNs are a broad category of machine learning algorithms that encompass many specific sub-categories. Various types of NNs exist, such as feedforward, radial, multilayer perceptron, convolutional, and recurrent, just to name a few. Here I focus on the use of a multi-layer perceptron (MLP) for the purpose of regression. All subsequent references to a NN are to a MLP regressor. A MLP has a minimum of three layers (input, hidden, and output). It is often used on data that cannot be linearly separated. A common representation of the MLP architecture is presented in Figure 38. 


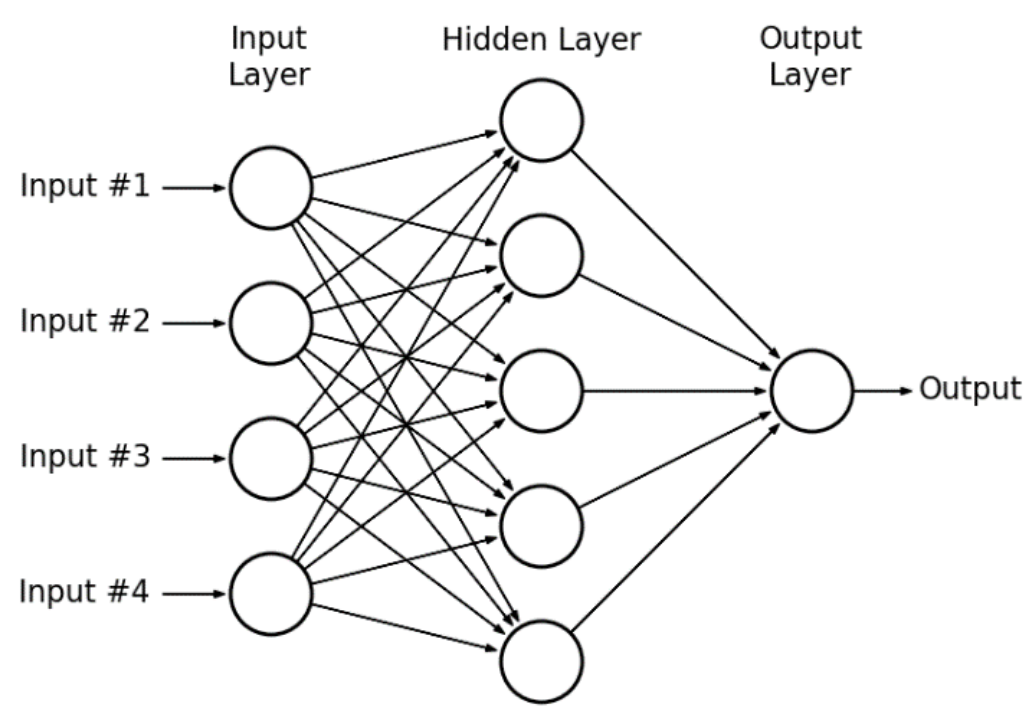

Figure 38. Common representation of a MLP NN [154].

In the MLP all the nodes (also called "neurons" or "perceptrons") in each layer connect to all the nodes in the subsequent layer. This type of network is known as feed-forward because inputs move forward through the model architecture. The interactions between neurons are a function of weights and biases. The MLP is trained with a technique known as backpropagation which adjusts the weights and biases of the neurons based on differences between the expected and predicted output values. Each of the neurons has a structure similar to the one presented in Figure 39 [155]. According to the Sci-Kit Learn documentation, the MLP advantages include the capability to learn non-linear models and to learn in real-time. Disadvantages include the risk of getting trapped in a local minimum solution, many hyper-parameters to tune and sensitivity to feature scaling [156]. There is a plethora of information forming on NNs in the online machine learning community and some of the deeper NN algorithms are not completely understood even by those who have developed them. Simple MLP regressors are well understood in terms of how they function but can sometimes act as a black box when they get too large. In depth analysis of the functioning of NNs was considered beyond the scope of this research. 


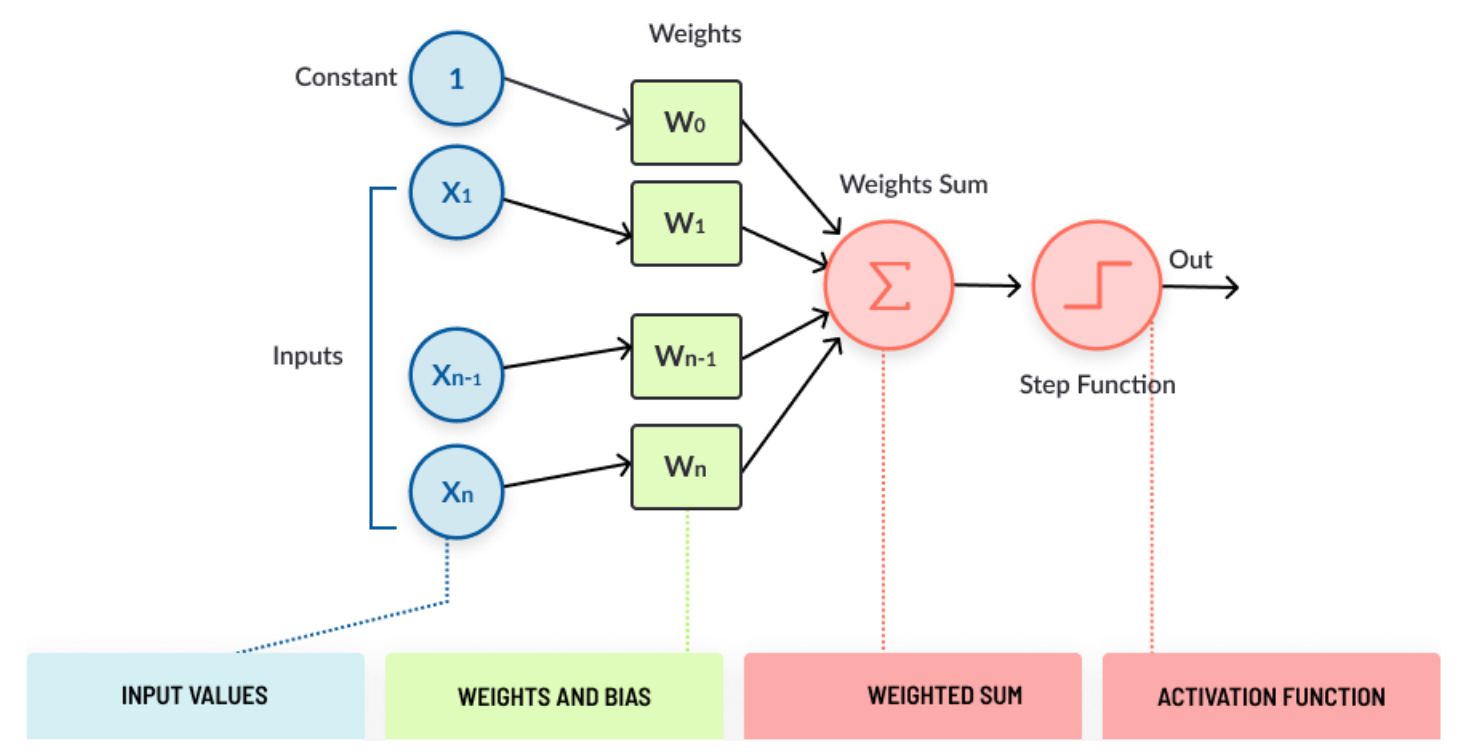

Figure 39. Individual MLP neuron description [155].

\subsubsection{Feature Reduction}

During the initial machine learning evaluations, it was clear that the number of features impacted the performance of the algorithms, particularly the NN. The general trend was that fewer features led to lower model prediction RMSEs. To evaluate the impact of the number of features on model performance, both the RF and the NN algorithms were evaluated by using a dataset that grew by one feature at a time. Features were added in order of their Pearson correlation with respect to release rate. So, the first feature was the one most strongly correlated with release rate, the algorithms were trained and tested, then the next strongest correlated feature was added and so on. This method resulted in 22 evaluations of both the NN and RF and allowed the number of features required for model optimization to be determined. The test set RMSEs as well as the percentage of the model minimum, as a function of the feature added are presented in Table 55.

One of the advantages of RFs is their ability to quantify the importance of different features, as previously discussed. RFs can produce attributes which define the feature importance for each variable in the dataset [157]. The feature importance is a percentage of the impact that each feature has, on average, on the RF's prediction. The default RF feature importance of each of the 22 features in the RRC-HFC dataset are presented in Table 55. 
Table 55. Impact on model RMSE of adding individual features successively.

\begin{tabular}{|l|c|c|c|c|c|}
\hline \multirow{2}{*}{ Feature Added } & \multicolumn{2}{|c|}{ NN } & \multicolumn{3}{c|}{ RF } \\
\cline { 2 - 6 } & $\begin{array}{c}\text { RMSE } \\
\text { (g/s) }\end{array}$ & $\begin{array}{c}\text { \% of } \\
\text { Minimum }\end{array}$ & $\begin{array}{c}\text { RMSE } \\
\text { (g/s) }\end{array}$ & $\begin{array}{c}\text { \% of } \\
\text { Minimum }\end{array}$ & $\begin{array}{c}\text { Feature } \\
\text { Importance }\end{array}$ \\
\hline $\mathrm{CH}_{4}$ Flux Random Error & 0.0571 & $99 \%$ & 0.0685 & $63 \%$ & $24.1 \%$ \\
\hline OTM Estimate & 0.0568 & $100 \%$ & 0.0604 & $72 \%$ & $10.7 \%$ \\
\hline OTM DQI & 0.0592 & $96 \%$ & 0.0600 & $72 \%$ & $0.8 \%$ \\
\hline OTM Avg. Bin $\mathrm{CH}_{4}$ & 0.0594 & $96 \%$ & 0.0576 & $75 \%$ & $1.6 \%$ \\
\hline $\mathrm{OTM} \mathrm{CH}_{4}$ Fit & 0.0662 & $86 \%$ & 0.0568 & $76 \%$ & $1.9 \%$ \\
\hline $\mathrm{CH}_{4}$ Spikes & 0.0630 & $90 \%$ & 0.0558 & $78 \%$ & $1.4 \%$ \\
\hline $\mathrm{CH}_{4}$ Flux & 0.0634 & $90 \%$ & 0.0549 & $79 \%$ & $3.2 \%$ \\
\hline Period Mean $\mathrm{CH}_{4}$ & 0.0676 & $84 \%$ & 0.0544 & $80 \%$ & $3.0 \%$ \\
\hline Mean LI-7700 Signal Strength & 0.0645 & $88 \%$ & 0.0504 & $86 \%$ & $6.3 \%$ \\
\hline $\mathrm{CH}_{4}$ Variance & 0.0628 & $90 \%$ & 0.0503 & $86 \%$ & $3.0 \%$ \\
\hline StS Direction & 0.0594 & $96 \%$ & 0.0481 & $90 \%$ & $2.7 \%$ \\
\hline $\mathrm{CH}_{4}$ Storage & 0.0642 & $88 \%$ & 0.0478 & $91 \%$ & $3.3 \%$ \\
\hline Air Temperature & 0.0675 & $84 \%$ & 0.0464 & $94 \%$ & $8.3 \%$ \\
\hline Distance & 0.0648 & $88 \%$ & 0.0461 & $94 \%$ & $1.2 \%$ \\
\hline Gaussian $\sigma_{\mathrm{y}}$ & 0.0697 & $81 \%$ & 0.0456 & $95 \%$ & $1.8 \%$ \\
\hline Direction of Maximum $\mathrm{CH}_{4}$ & 0.0680 & $83 \%$ & 0.0459 & $95 \%$ & $1.5 \%$ \\
\hline Air Heat Capacity & 0.0741 & $77 \%$ & 0.0435 & $100 \%$ & $10.5 \%$ \\
\hline Quality Control $\mathrm{CH}_{4}$ Flux & 0.0755 & $75 \%$ & 0.0434 & $100 \%$ & $0.3 \%$ \\
\hline Anemometer Yaw & 0.0750 & $76 \%$ & 0.0449 & $97 \%$ & $5.1 \%$ \\
\hline $\mathrm{CH}_{4}$ Max Angle & 0.0735 & $77 \%$ & 0.0446 & $97 \%$ & $1.5 \%$ \\
\hline Anemometer Pitch & 0.0739 & $77 \%$ & 0.0446 & $97 \%$ & $4.1 \%$ \\
\hline Temperature Variance & 0.0848 & $67 \%$ & 0.0453 & $96 \%$ & $3.7 \%$ \\
\hline
\end{tabular}

The NN attained its best results with the use of just two features with an RMSE of $0.0568 \mathrm{~g} / \mathrm{s}$. Though it should be noted that the network used here was the default and had only one hidden layer of size 100, a different architecture could have significantly changed results. The RF achieved its lowest RMSE with the use of the first 18 features with an RMSE of $0.0434 \mathrm{~g} / \mathrm{s}$. These results further emphasize the importance of input features as optimal selection reduced the difference between the two models from $61 \%$ to $27 \%$. A comparison of the performance of the two models with their minimum features $(\mathrm{NN}=2, \mathrm{RF}=18)$ is presented in Table 56.

Table 56. Effects of variable reduction on RF and NN default models.

\begin{tabular}{|c|c|c|c|c|}
\hline \multirow{2}{*}{ Model } & \multicolumn{2}{|c|}{ Controlled Release Test Set RMSE(g/s) } & \multicolumn{2}{c|}{ MSEEL Audit RMSE (g/s) } \\
\cline { 2 - 5 } & Default & Min Features & Default & Min Features \\
\hline RF & 0.045 & 0.043 & 0.22 & 0.22 \\
\hline NN & 0.085 & 0.057 & 0.49 & 0.95 \\
\hline
\end{tabular}


The minimal feature RF and NN models improved upon their default RMSEs by $5 \%$ and $33 \%$, respectively, for the controlled release test dataset. However, neither model improved on their predictions on the audit dataset, which was much less like the training data. The RF model did not perform any worse on the MSEEL audit set, whereas the NN MSEEL RMSE increased by 94\% suggesting that the robustness of the model was severely limited by only utilizing two features. To compensate for this the 18 features identified by the $\mathrm{RF}$ feature analysis were used in both models moving forward as the default feature set. Using the RF identified features on the NN decreased the test RMSE by $13 \%$ from the default value $0.085 \mathrm{~g} / \mathrm{s}$ to the new value of $0.074 \mathrm{~g} / \mathrm{s}$ and did not affect the MSEEL RMSE. Descriptions of these features and their statistics are presented in Table 57. 
Table 57. Descriptions and scaling statistics of final model input features.

\begin{tabular}{|c|c|c|c|c|c|c|c|}
\hline Feature Added & Source & Units & Description & $\begin{array}{c}\mathrm{NaN} \\
(\#)\end{array}$ & $\begin{array}{c}\text { Outliers } \\
\text { (\#) }\end{array}$ & $\begin{array}{l}\text { Mean } \\
\text { (units) }\end{array}$ & Std.(units) \\
\hline $\begin{array}{l}\mathrm{CH}_{4} \text { Flux Random } \\
\text { Error }\end{array}$ & EddyPro & $\left(\mu \mathrm{mol} / \mathrm{m}^{2} \mathrm{~s}\right)$ & Random error for methane flux (F\&S) & 48 & 246 & 0.042 & 0.07 \\
\hline OTM Estimate & OTM & $(\mathrm{g} / \mathrm{s})$ & OTM mass emission estimate & 0 & 227 & 0.043 & 0.09 \\
\hline OTM DQI & OTM & $(\#)$ & OTM Data Quality Indicator & 0 & 0 & 19 & 8.53 \\
\hline $\begin{array}{l}\text { OTM Avg. Bin } \\
\mathrm{CH}_{4}\end{array}$ & OTM & $(\mathrm{ppm})$ & $\begin{array}{l}\text { Average Concentration above background of all fitted OTM } \\
\text { bins. }\end{array}$ & 0 & 213 & 0.11 & 0.22 \\
\hline $\mathrm{OTM} \mathrm{CH}_{4}$ Fit & OTM & $(\mathrm{ppm})$ & Peak methane concentration above background of OTM bins. & 0 & 229 & 0.20 & 0.54 \\
\hline $\mathrm{CH}_{4}$ Spikes & EddyPro & $(\#)$ & Number of methane spikes in the period. & 0 & 399 & 0.68 & 1.58 \\
\hline $\mathrm{CH}_{4}$ Flux & EddyPro & $\left(\mu \mathrm{mol} / \mathrm{m}^{2} \mathrm{~s}\right)$ & Methane flux. & 0 & 215 & 0.13 & 0.88 \\
\hline Mean $\mathrm{CH}_{4}$ & Both & $(\mathrm{ppm})$ & Mean methane concentration & 0 & 245 & 2.3 & 0.43 \\
\hline $\begin{array}{l}\text { Mean LI-7700 } \\
\text { Signal Strength }\end{array}$ & EddyPro & $(\%)$ & The mean LI-7700 signal strength of the period. & 0 & 152 & 32 & 16.38 \\
\hline $\mathrm{CH}_{4}$ Variance & EddyPro & $\left(\mathrm{ppm}^{2}\right)$ & Methane concentration variance. & 0 & 465 & 0.00024 & 0.00 \\
\hline StS Direction & Measured & $\left({ }^{\circ}\right)$ & Source to Sensor direction. & 0 & 0 & 247 & 16.93 \\
\hline $\mathrm{CH}_{4}$ Storage & EddyPro & $\left(\mu \mathrm{mol} / \mathrm{m}^{2} \mathrm{~s}\right)$ & Methane storage. & 73 & 92 & $\begin{array}{c}- \\
0.000090\end{array}$ & 0.00 \\
\hline $\begin{array}{l}\text { Mean Air } \\
\text { Temperature }\end{array}$ & Both & $(\mathrm{K})$ & Average Ambient Temperature. & 0 & 0 & 296 & 5.47 \\
\hline Distance to Source & Measured & $(\mathrm{m})$ & Distance from the source to the sensor. & 0 & 0 & 64 & 27.27 \\
\hline Gaussian $\sigma_{\mathrm{y}}$ & OTM & $(\mathrm{m})$ & $\begin{array}{l}\text { OTM estimated Gaussian Spread (perpendicular to wind } \\
\text { direction) }\end{array}$ & 0 & 165 & 9.4 & 4.99 \\
\hline $\begin{array}{l}\text { Direction of } \\
\text { Maximum } \mathrm{CH}_{4}\end{array}$ & OTM & $\left({ }^{\circ}\right)$ & Direction of Peak methane concentration Bin & 0 & 12 & 184 & 39.54 \\
\hline $\begin{array}{l}\text { Average Air Heat } \\
\text { Capacity }\end{array}$ & EddyPro & $(\mathrm{J} / \mathrm{K}-\mathrm{kg})$ & Average air heat capacity. & 0 & 0 & 1014 & 5.94 \\
\hline $\begin{array}{l}\text { Quality Control } \\
\mathrm{CH}_{4} \text { Flux }\end{array}$ & EddyPro & $(\#)$ & EC quality control rating (Mauder and Foken, 2004). & 0 & 0 & 0.96 & 0.75 \\
\hline
\end{tabular}




\subsubsection{Model Improvements}

A low-cost approach to improve machine learning algorithms is hyper-parameter optimization. To improve the performance of the RF and NN models, the controlled-release dataset was analyzed while tuning the model hyper-parameters. There are several methods for hyper-parameter tuning which can be utilized depending on the hyper-parameter search space size, computational power, and relevance. A random grid search was used to optimize the parameters of both the RF and the NN. Random searches have been proven to be more efficient at finding optimal hyper-parameters than a strict grid search, in which all possible iterations are tested. Random searches use random combinations of the hyper-parameters in question which speeds up the total search time compared to evaluating each potential combination with minimal loss of improvement [158].

The random search with cross-fold validation was utilized for hyperparameter search optimization. The search was evaluated for 100 iterations and 3 cross-fold validations [159]. Cross-fold validation works by segmenting the training set into equally sized blocks. For each iteration of training one of the blocks is set aside for validation, while the model is trained on the other two blocks. The number of iterations indicates the total number of random parameter combinations evaluated. During each of the iterations, cross-fold validation is performed on the model with the defined hyper-parameters of the iteration. In total the number of models trained and validated based on the training set during this method was 300 (iterations * crossfolds).

A random search was utilized to optimize the critical hyper-parameters of the RF model. The parameters evaluated and how each one affects the RF, along with the values used to form the random grid search matrix are presented in Table 58. The total number of possible hyper-parameter combinations was 3960, so utilizing the random search reduced the number of required computations by an order of magnitude.

Table 58. Hyper-parameters used for RF random search grid [157].

\begin{tabular}{|c|c|l|c|}
\hline Parameter & $\begin{array}{c}\text { Default } \\
\text { Value }\end{array}$ & \multicolumn{1}{|c|}{ Description } & Other Test Values \\
\hline n_estimators & 100 & The number of trees in the forest. & $10,1000,10000$ \\
\hline min_samples_split & 2 & $\begin{array}{l}\text { The minimum number of samples required to split } \\
\text { an internal node. }\end{array}$ & $4,6,8,10$ \\
\hline min_samples_leaf & 1 & $\begin{array}{l}\text { The minimum number of samples required to be at } \\
\text { a leaf node. }\end{array}$ & $3,5,7,9,11$ \\
\hline max_features & 'auto' & $\begin{array}{l}\text { The number of features to consider when looking } \\
\text { for the best split. ('auto' utilizes all available } \\
\text { features) }\end{array}$ & 'sqrt', 'log2' \\
\hline ccp_alpha & 0 & $\begin{array}{l}\text { Complexity parameter used for Minimal Cost- } \\
\text { Complexity Pruning. By default, no pruning is } \\
\text { performed. }\end{array}$ & $\begin{array}{c}{\left[1.0 \times 10^{-5}: 1.0 \times 10^{-5}:\right.} \\
\left.1.0 \times 10^{-4}\right]\end{array}$ \\
\hline
\end{tabular}

The results of the randomized search, however, did not improve the RF model performance. Therefore, the default model was maintained as the model used. An analysis of each of the parameters individually, in which only one was varied at a time confirmed that the default values produced the lowest RMSE on all parameters except for the number of estimators. An order of magnitude increase in the number of estimators only reduced the test set RMSE by $0.14 \%$ and came at the expense of an order of magnitude of computational time, therefore the default number of estimations $(n=100)$ was maintained. Since the 
random search grid did not produce a significantly improved RMSE, the default RF was used as the final model, which when trained on the controlled release dataset resulted in a test RMSE of $0.043 \mathrm{~g} / \mathrm{s}$ and an MSEEL audit RMSE of $0.22 \mathrm{~g} / \mathrm{s}$.

Like the RF, an attempt to optimize NN hyper-parameters was also completed. The NN showed more promise as a global method than the linear regressor, as previously discussed. It was also well documented in literature that deep NNs are applicable to complex problems for both regression and classification. For consistency, the same random search with cross-validation was utilized for hyper-parameter tuning of the NN. Scaled features were once again used from the controlled release dataset and the RF identified features made up the inputs to the MLP regressor.

NNs have an infinite number of hyper-parameters because the number of layers and the number of neurons per layer can be set to any number. For the sake of minimizing the search space the number of layers tested and the size of those layers were limited. Equation 30 was utilized to determine a search space for number of total neurons based on recommendations [160].

$$
N_{\text {hidden neurons }}=\frac{N_{\text {training samples }}}{\left(\alpha *\left(N_{\text {inputs }}+N_{\text {outputs }}\right)\right)}
$$

Equation 30

Where $\alpha$ is a scaling factor between 2 and 10. Based on the controlled release dataset the number of training samples was 1206, the number of inputs was 18 , and the number of outputs was 1 . The $\alpha$ value was varied to produce a test array. The values used for $\alpha$ were $2,4,6,8$, and 10. The total number of layers over which to distribute these neurons was also required. Researchers have found that deeper NNs do not necessarily improve results for many problems. To keep the number of hidden layers to a minimum, up to three hidden layers were tested. The total number of hidden neurons for each of the different $\alpha$ levels were distributed evenly amongst the number of hidden layers. As a result, the number and size of hidden layers tested in the random search were those presented in Table 59.

\section{Table 59. Hidden layer sizes used for NN hyper-parameters.}

\begin{tabular}{|c|c|c|c|}
\hline \multirow{2}{*}{$\begin{array}{c}\text { Total } \\
\text { Hidden } \\
\text { Neurons (\#) }\end{array}$} & \multicolumn{3}{|c|}{ Hidden Layers (\#) } \\
\cline { 2 - 4 } & $\mathbf{1}$ & $\mathbf{2}$ & $\mathbf{3}$ \\
\hline $\mathbf{6}$ & 6 & 3 & 2 \\
\hline $\mathbf{7}$ & 7 & 4 & 2 \\
\hline $\mathbf{1 0}$ & 10 & 5 & 3 \\
\hline $\mathbf{1 5}$ & 15 & 8 & 5 \\
\hline $\mathbf{3 1}$ & 31 & 16 & 10 \\
\hline
\end{tabular}

In addition to these hidden layer shapes, the other hyper-parameters tested in the initial evaluation were the activation function, solver, and L2 penalty (alpha). The values utilized in the initial random grid search are presented in Table 60 . The alpha values tested were five evenly spaced values between $1.0 \times 10^{-5}$ and 1 on a log-axis. 
Table 60. Hyper-parameters used for NN random search grid [161].

\begin{tabular}{|c|c|c|c|}
\hline Parameter & Default Value & Description & Other Test Values \\
\hline hidden_layer_sizes & $(100)$, & $\begin{array}{c}\text { The ith element represents the total } \\
\text { number of neurons in the ith hidden } \\
\text { layer. }\end{array}$ & Defined by Table 59 \\
\hline activation & 'relu' & $\begin{array}{c}\text { Activation function for hidden } \\
\text { layer. }\end{array}$ & 'identity', 'logistic', 'tanh' \\
\hline solver & 'adam' & The solver for weight optimization. & 'lbfgs', 'sgd' \\
\hline alpha & 'auto' & $\begin{array}{c}\text { L2 penalty (regularization term) } \\
\text { parameter. }\end{array}$ & {$\left[1.0 \times 10^{-5}: 1\right]$} \\
\hline
\end{tabular}

The search was evaluated for 100 iterations and 3 cross-fold validations, which reduced the total number of required iterations by a factor of 3 . The resultant randomized search parameters resulted in a $20 \%$ decrease in the test set RMSE. The optimal randomized search parameters are presented in Table 61 . The optimized activation function was the rectified linear unit (relu) function. This function is commonly used in NNs and takes the form of Equation 31.The resultant optimal solver was a limited-memory BroydenFletcher-Goldfard-Shanno (lbfgs). This is a type of quasi-Newton optimization algorithm for finding local minima or maxima of smaller datasets [161].

$$
f(x)=\max (0, x)
$$

Equation 31

A second random search was performed to optimize secondary parameters related to the solver. These features included the maximum number of iterations ('max_iter', default $=200$ ) and the maximum number of function calls ('max_fun', default $=15000$ ). These parameters were tested with the optimal results from the first iteration random search. The 'max_iter' parameter was evaluated on 10 linearly spaced values between 10 and 1000, and the 'max_fun' parameter was evaluated on 10 linearly spaced values between 1000 and 100,000. The random grid for this case tested all 100 possible combinations on three cross-fold validations to determine the optimized hyper-parameters. The optimal values of 'max_iter' and 'max_fun' were determined to be 450 and 1000, respectively. However, changing these values only reduced the RMSE of the test set by less than $1 \%$. The final NN, which was optimized for the controlled release dataset, was constructed with the settings in Table 61 . This was a $10 \%$ reduction in RMSE over the minimum feature evaluation RMSE using only two features. The resultant model was also believed to be more robust and less prone to overfitting, based on the differences in the MSEEL dataset evaluation.

Table 61. NN optimized hyper-parameters.

\begin{tabular}{|c|c|}
\hline NN Parameter & Optimal Value \\
\hline $\begin{array}{c}\text { hidden_layer_sizes } \\
\text { (layers of neurons, }\end{array}$ & $(10)$, \\
\hline activation & relu \\
\hline solver & lbfgs \\
\hline alpha (\#) & 0.056 \\
\hline max_iter (\#) & 450 \\
\hline max_fun (\#) & 1000 \\
\hline RMSE (g/s) & 0.051 \\
\hline
\end{tabular}


Both the RF and NN models utilized a randomized grid search with cross-fold validation to optimize the hyper-parameters used. The RF improvements were minimal with these searches while the NN model improved by more than $32 \%$ when compared to the default model with the RF optimized feature set on the controlled release test dataset. The final models used were those that resulted in the minimized RMSE without compromising computational time and expense. The improvements in both models are presented in Table 62.

Table 62. RF and NN model iterations.

\begin{tabular}{|l|c|c|}
\hline \multirow{2}{*}{\multicolumn{1}{|c|}{ Features/Model Change }} & \multicolumn{2}{c|}{$\begin{array}{c}\text { Controlled } \\
\text { (g/s) }\end{array}$} \\
\cline { 2 - 3 } & $\mathbf{R F}$ & $\mathbf{N N}$ \\
\hline RRC-HCF & 0.0453 & 0.085 \\
\hline Minimum Features & 0.0434 & 0.057 \\
\hline RF-Optimized Features & 0.0434 & 0.075 \\
\hline Hyper-parameter Tuning 1 & 0.0455 & 0.052 \\
\hline Hyper-parameter Tuning 2 & - & 0.051 \\
\hline Final & 0.0434 & 0.051 \\
\hline Feature Set & RF Optimized & RF Optimized \\
\hline Number of Features (\#) & 18 & 18 \\
\hline Tuning Iterations (\#) & 0 & 2 \\
\hline
\end{tabular}

\section{Results}

\subsection{Controlled Releases}

The final RF and NN models were compared to the OTM and EC methods on the valid EC footprint periods from 4.2. Both models outperformed both OTM and EC footprint across the 142-period test set. It should be noted that some of the periods were likely used in the training sets of the models, although since the sets were randomized the fraction of the periods used for training is unknown. A comparison of the RMSEs and mean absolute percent error (MAPE) from all methods are presented in Table 63. Figure 40, Figure 41, and Figure 42 present the different release rates for these shared periods (note the difference in scales). It is again important to note that over $98 \%$ of these periods were recorded during a release distance of $42 \mathrm{~m}$. The sample sizes for the release rates of $0.04,0.12$, and $0.24 \mathrm{~g} / \mathrm{s}$ were 87,41 , and 14 , respectively. The box data are the predictions from the given method. The boxes encapsulate the lower and upper quartiles, the whiskers extend to the $5^{\text {th }}$ and $95^{\text {th }}$ percentiles, the blue lines represent the means, the magenta lines represent the medians, dotted green lines represent the actual release rate, dotted orange lines represent the estimate H\&R uncertainty of EC footprint estimates, the red dotted lines represent the minimum achievable OTM uncertainties based on the analysis presented previously. The statistics of the data presented in the figures are presented in Table 64. The RF method performed the best across all release rates, with a total RMSE of just $0.018 \mathrm{~g} / \mathrm{s}$. This RMSE was $50 \%$ lower than the NN and more than $68 \%$ lower than any of the traditional methods. 
Table 63. Estimate comparisons of valid EC periods.

\begin{tabular}{|c|c|c|}
\hline Model/Method & RMSE (g/s) & MAPE (\%) \\
\hline FFP Footprint & 0.087 & $84 \%$ \\
\hline KM Footprint & 0.080 & $83 \%$ \\
\hline Taguchi OTM & 0.058 & $44 \%$ \\
\hline NN Optimized & $\mathbf{0 . 0 3 6}$ & $\mathbf{4 5 \%}$ \\
\hline RF Optimized & $\mathbf{0 . 0 1 8}$ & $\mathbf{1 6 \%}$ \\
\hline
\end{tabular}

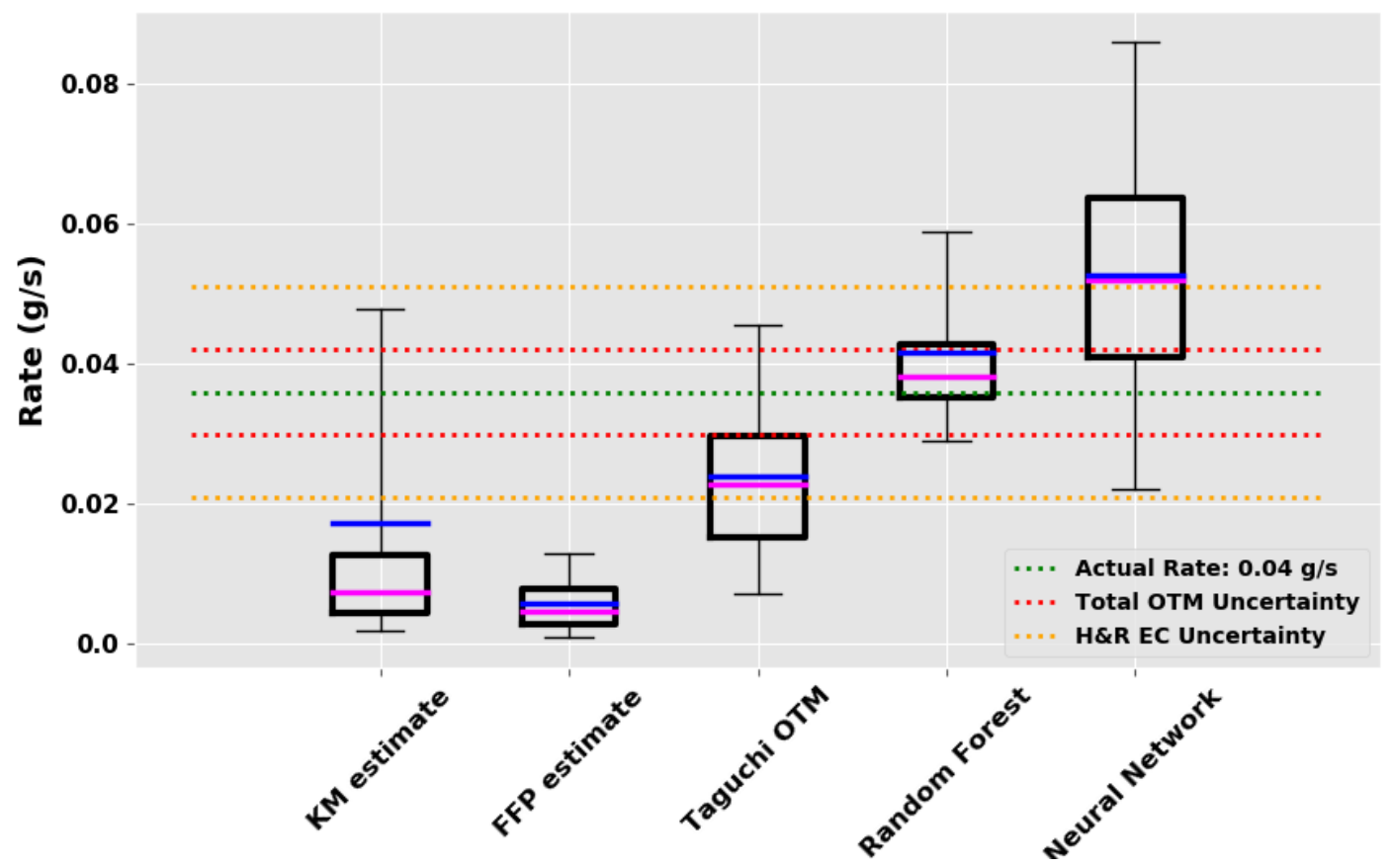

Figure 40. Comparison of method results for valid EC periods with a release rate of $0.04 \mathrm{~g} / \mathrm{s}$. 


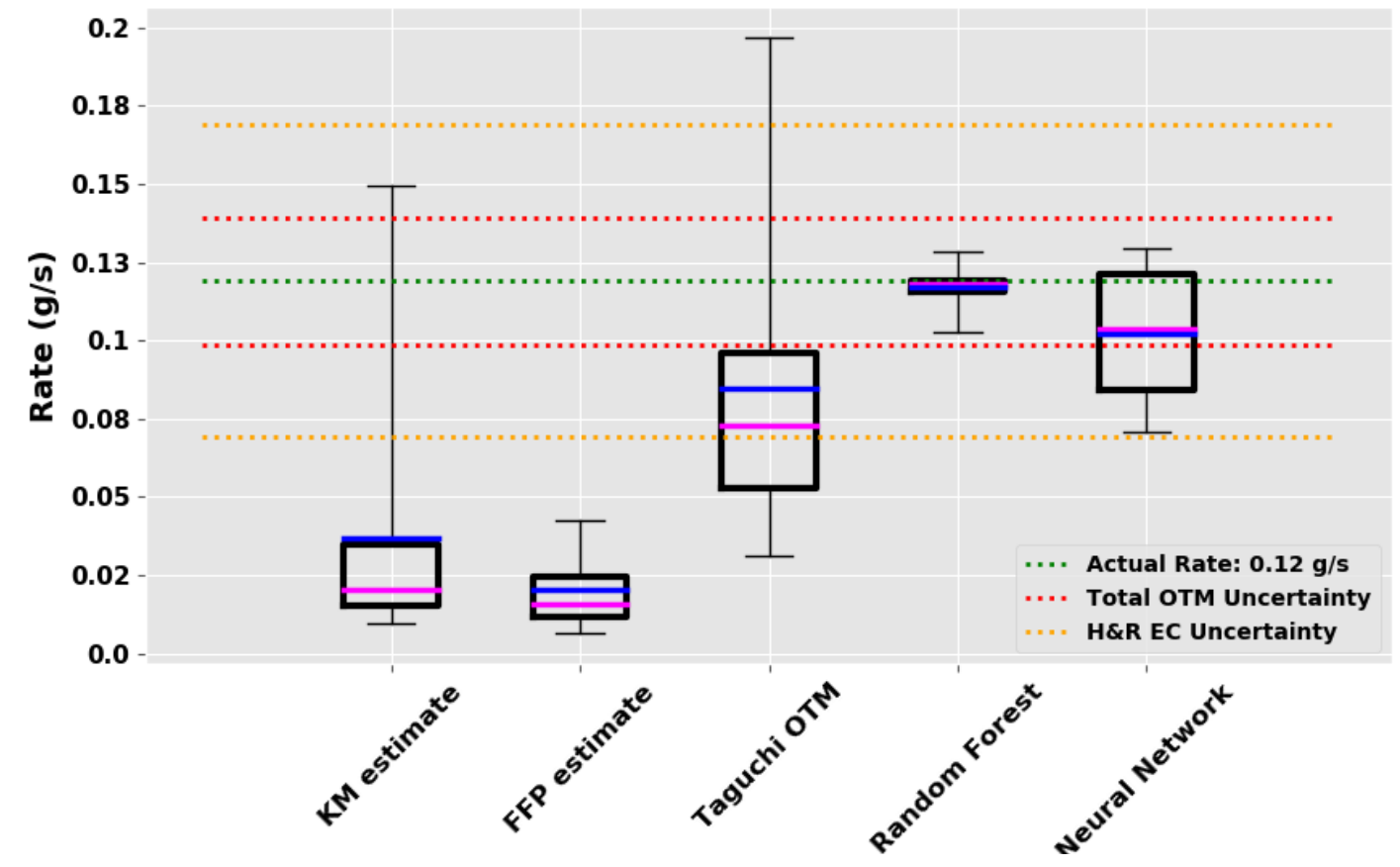

Figure 41. Comparison of method results for valid EC periods with a release rate of $0.12 \mathrm{~g} / \mathrm{s}$.

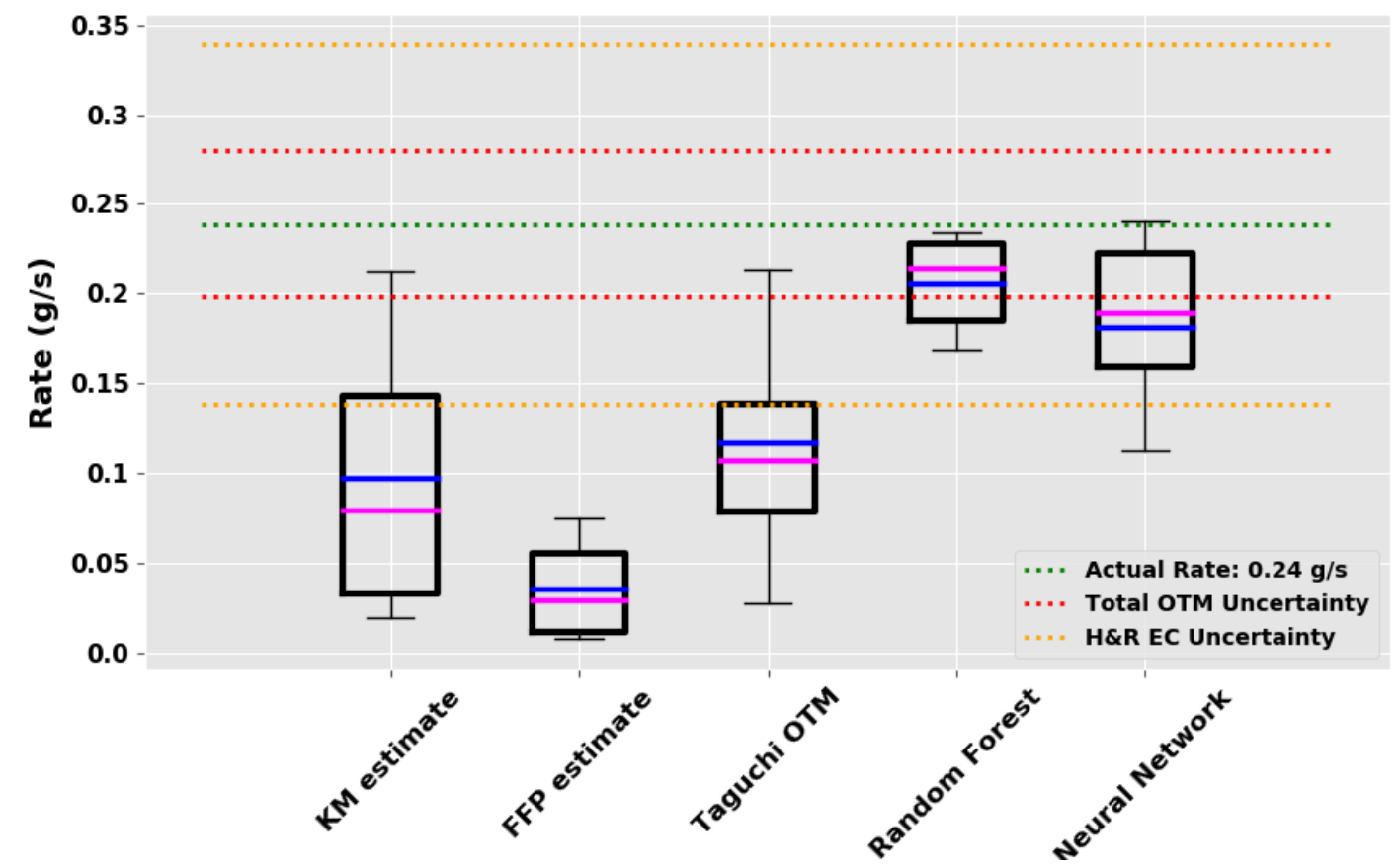

Figure 42. Comparison of method results for valid EC periods with a release rate of $0.24 \mathrm{~g} / \mathrm{s}$. 
Table 64. Summary statistics of valid EC periods from all estimation methods.

\begin{tabular}{|c|c|c|c|c|c|}
\hline Method & KM & FFP & OTM & RF & NN \\
\hline \multicolumn{7}{|c|}{$\mathbf{0 . 0 3 6} \mathbf{g} / \mathbf{s}, \mathbf{n}=\mathbf{8 7}$} \\
\hline Q1 & 0.0044 & 0.0029 & 0.015 & 0.035 & 0.041 \\
\hline Mean & 0.017 & 0.0058 & 0.024 & 0.042 & 0.053 \\
\hline Median & 0.0073 & 0.0047 & 0.023 & 0.038 & 0.052 \\
\hline Q3 & 0.013 & 0.0078 & 0.030 & 0.043 & 0.064 \\
\hline \multicolumn{7}{|c|}{$\mathbf{0 . 1 1 9} \mathbf{g} / \mathbf{s}, \mathbf{n}=\mathbf{4 1}$} \\
\hline Q1 & 0.015 & 0.011 & 0.053 & 0.12 & 0.08 \\
\hline Mean & 0.037 & 0.020 & 0.085 & 0.12 & 0.10 \\
\hline Median & 0.020 & 0.016 & 0.073 & 0.12 & 0.10 \\
\hline Q3 & 0.035 & 0.024 & 0.10 & 0.12 & 0.12 \\
\hline \multicolumn{7}{|c|}{$\mathbf{0 . 2 3 9} \mathbf{g} / \mathbf{s}, \mathbf{n}=\mathbf{1 4}$} \\
\hline Q1 & 0.032 & 0.011 & 0.078 & 0.19 & 0.16 \\
\hline Mean & 0.10 & 0.04 & 0.12 & 0.21 & 0.18 \\
\hline Median & 0.080 & 0.030 & 0.11 & 0.21 & 0.19 \\
\hline Q3 & 0.14 & 0.055 & 0.14 & 0.23 & 0.22 \\
\hline
\end{tabular}

In order to increase the dataset, it was desirable to have a method that could be utilized on periods that could not produce a valid EC estimate. An ideal model would be able to produce results under all micrometeorological conditions. Two other comparisons were made between the machine learning models and the OTM results. The EC footprint methods could not be compared across all these periods because valid mass rate estimates were not always attainable under the given conditions. These periods were not filtered by any criteria other than the WF that was initially used. The complete release rate dataset of 804 periods and the machine learning test dataset of 402 periods were evaluated against the default and Taguchi OTM results. The RMSEs of the 402 periods in the test set were compared from the machine learning models in Table 62. The default and Taguchi OTM RMSEs for this test set were $0.12 \mathrm{~g} / \mathrm{s}$ and $0.13 \mathrm{~g} / \mathrm{s}$, respectively. The default OTM did outperform the Taguchi on this limited set, which was further indication that the OTM method results are at the mercy of the conditions of the period. The uncertainty of the RF was also evaluated on this test set. The IJ method was utilized to estimate the uncertainty of each of the predictions. The IJ function utilizes the training and testing datasets and the final model of the RF to estimate a standard deviation of each estimate based on a bootstrapped, bagging method. The results were subdivided into release rate. Figure 43 presents the average prediction of the RF model and average standard deviation using the IJ method for each of the 402 periods in the test set. The blue lines represent the mean prediction of the periods with the known release rate and the error bars extend to the mean standard deviation of those periods based on the IJ. The red dotted line represents the identity function. 


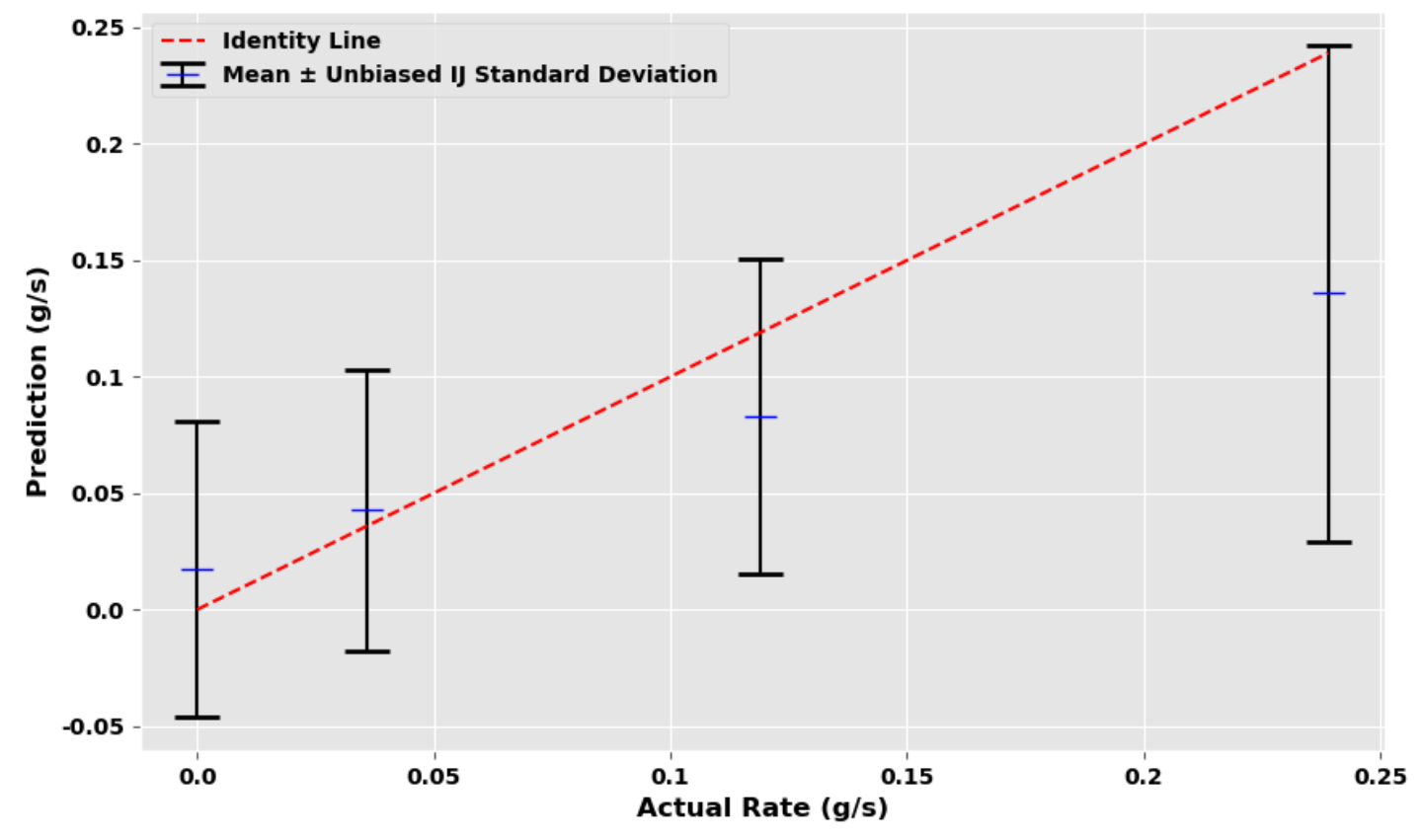

Figure 43. RF test dataset mean IJ uncertainty.

The complete controlled release dataset was also analyzed from the perspective of both machine learning techniques and both OTM calculations. The RMSE results were calculated for the entire controlled release dataset ( 804 periods). The Taguchi OTM RMSE was reduced by $27 \%$ over the default OTM. The NN and RF improved upon the Taguchi OTM by an average of $44 \%$ and $69 \%$, respectively. The RMSEs by release rate and method are presented in Table 65 . The RF RMSE was $44 \%$ less on average than the NN across the three releases. Figure 44, Figure 45, and Figure 46 present the different release rates for these shared periods (note the difference in scales). The sample sizes for the release rates of $0.04,0.12$, and $0.24 \mathrm{~g} / \mathrm{s}$ were 395 , 325 , and 84 , respectively. The box data are the predictions from the given method. The boxes encapsulate the lower and upper quartiles, the whiskers extend to the $5^{\text {th }}$ and $95^{\text {th }}$ percentiles, the blue lines represent the means, the magenta lines represent the medians, dotted green lines represent the actual release rate, and the red dotted lines represent the minimum achievable OTM uncertainties based on the analysis presented previously. The statistics are presented in Table 66.

Table 65. RMSE results of full control release dataset for default and Taguchi OTM, RF, and NN.

\begin{tabular}{|c|c|c|c|c|c|}
\hline $\begin{array}{c}\text { Release Rate } \\
(\mathbf{g} / \mathbf{s})\end{array}$ & Count & $\begin{array}{c}\text { Default } \\
\text { OTM }\end{array}$ & $\begin{array}{c}\text { Taguchi } \\
\text { OTM }\end{array}$ & $\begin{array}{c}\text { Random } \\
\text { Forest }\end{array}$ & $\begin{array}{c}\text { Neural } \\
\text { Network }\end{array}$ \\
\hline 0.036 & 395 & 0.059 & 0.038 & 0.012 & 0.024 \\
\hline 0.119 & 325 & 0.19 & 0.17 & 0.030 & 0.054 \\
\hline 0.239 & 84 & 0.25 & 0.17 & 0.073 & 0.12 \\
\hline
\end{tabular}




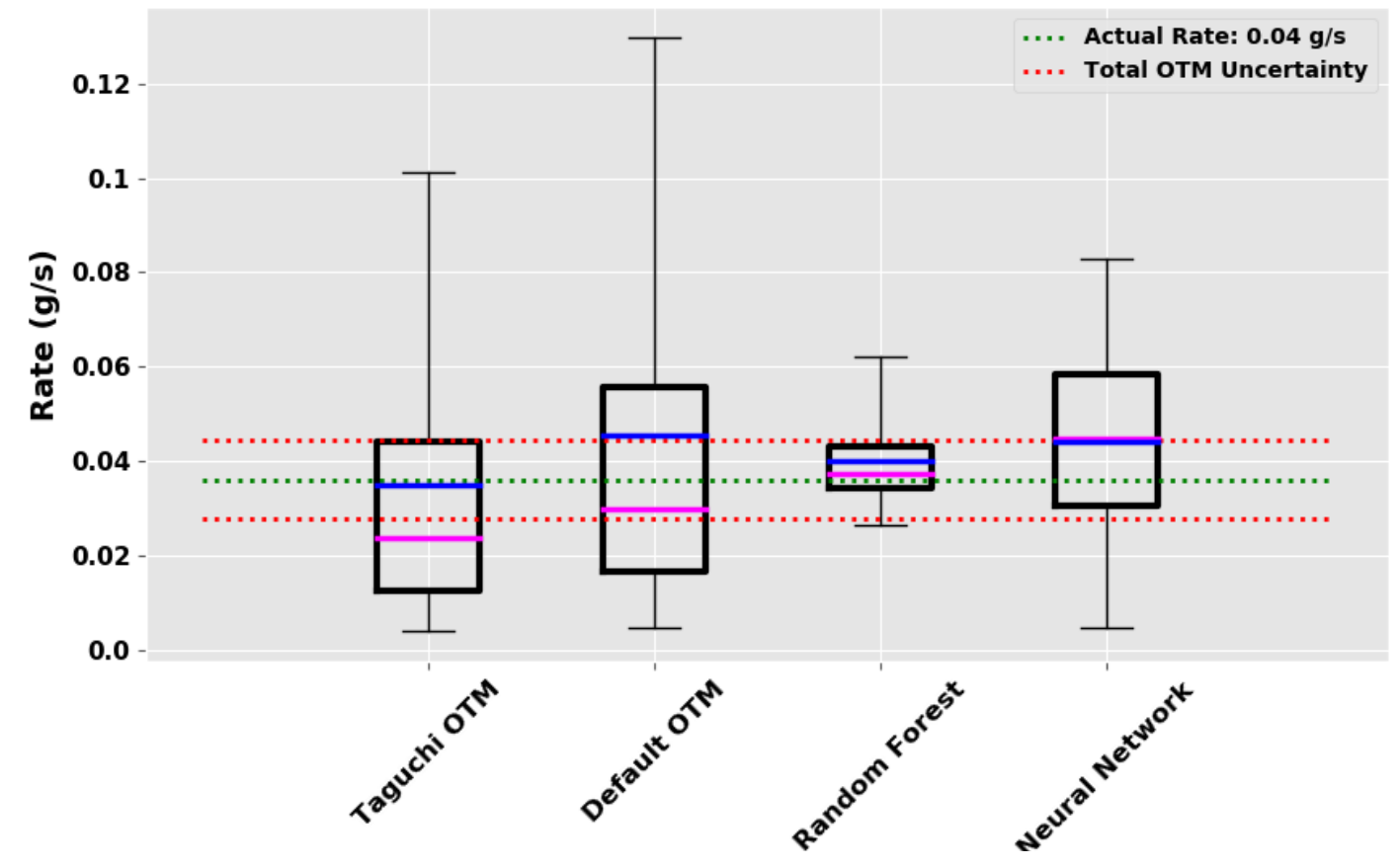

Figure 44. Comparison of default and Taguchi OTM, RF, and NN on all periods (0.04 g/s).

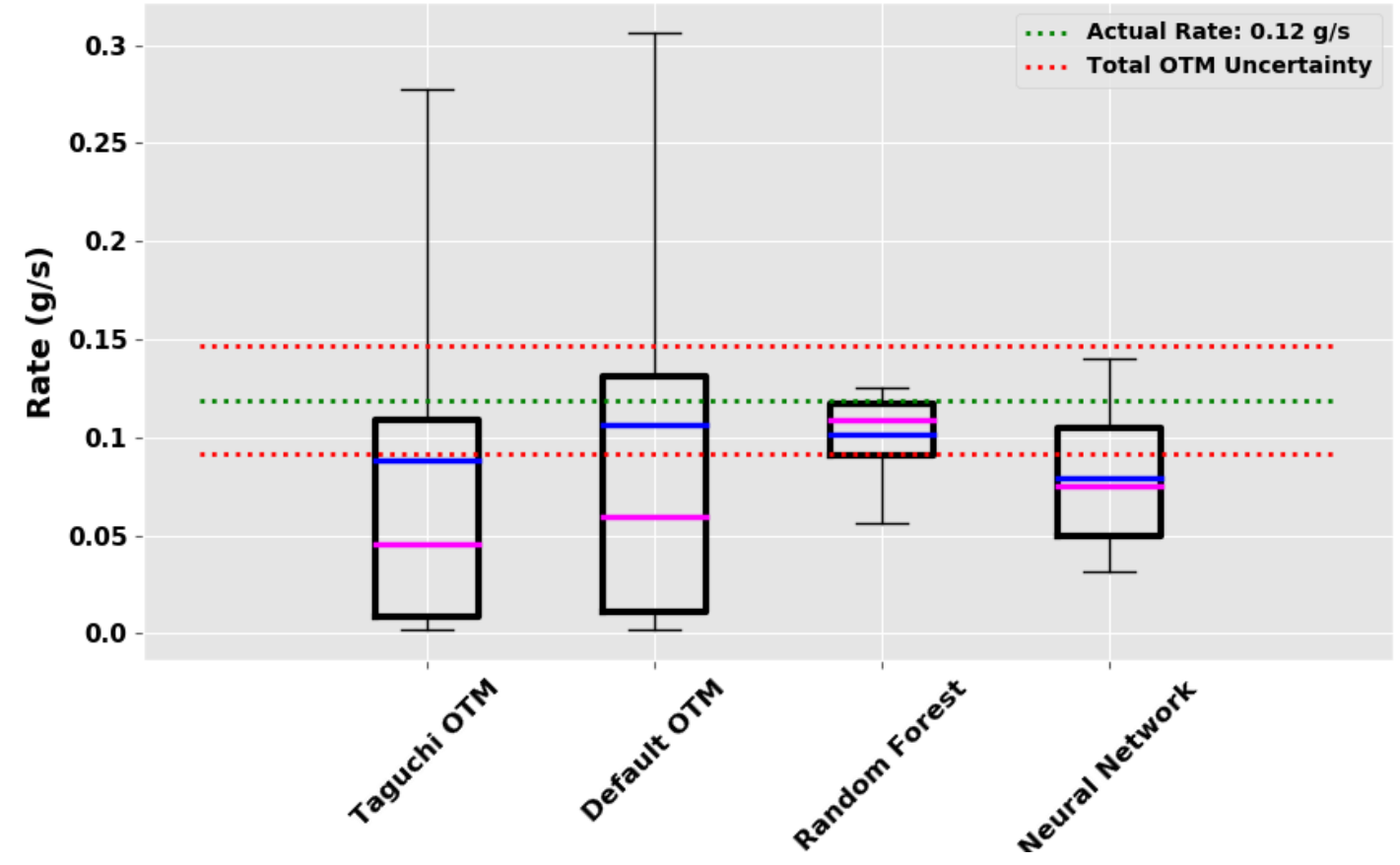

Figure 45. Comparison of default and Taguchi OTM, RF, and NN on all periods (0.12 $\mathrm{g} / \mathrm{s})$. 


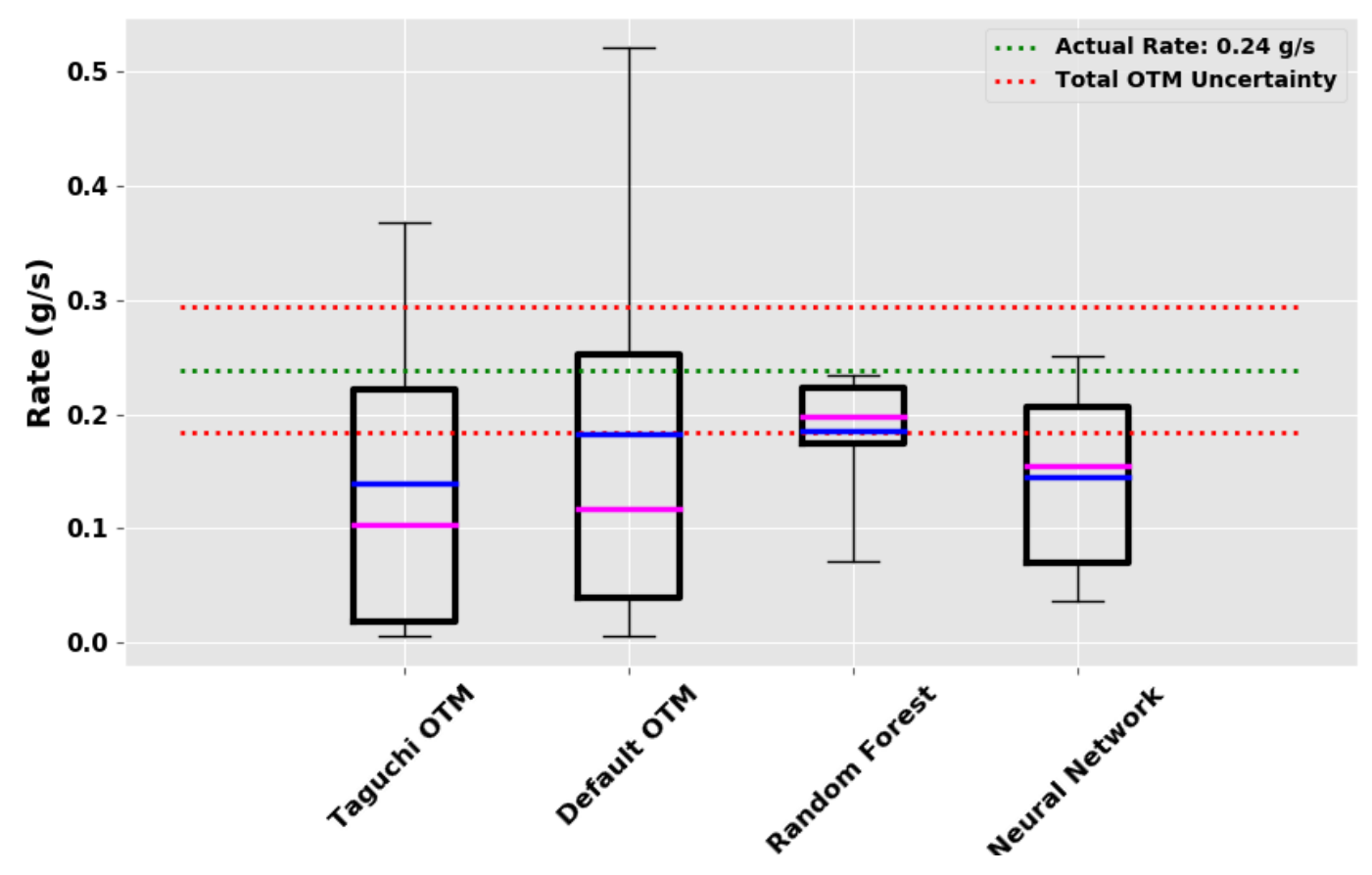

Figure 46. Comparison of default and Taguchi OTM, RF, and NN on all periods (0.24 g/s).

Table 66. Statistics of predicted rates (g/s) for default and Taguchi OTM, RF, and NN.

\begin{tabular}{|c|c|c|c|c|}
\hline Model & $\begin{array}{c}\text { Default } \\
\text { OTM }\end{array}$ & $\begin{array}{c}\text { Taguchi } \\
\text { OTM }\end{array}$ & RF & NN \\
\hline \multicolumn{5}{|c|}{$\mathbf{0 3 6} \mathbf{g} / \mathbf{s}, \mathbf{n}=\mathbf{3 9 5}$} \\
\hline Q1 & 0.017 & 0.012 & 0.034 & 0.030 \\
\hline Mean & 0.046 & 0.035 & 0.040 & 0.044 \\
\hline Median & 0.030 & 0.024 & 0.037 & 0.045 \\
\hline Q3 & 0.056 & 0.044 & 0.043 & 0.058 \\
\hline \multicolumn{5}{|c|}{$\mathbf{0 . 1 1 9} \mathbf{g} / \mathbf{s}, \mathbf{n}=\mathbf{3 2 5}$} \\
\hline Q1 & 0.011 & 0.0087 & 0.091 & 0.050 \\
\hline Mean & 0.11 & 0.088 & 0.10 & 0.079 \\
\hline Median & 0.059 & 0.045 & 0.11 & 0.075 \\
\hline Q3 & 0.13 & 0.11 & 0.12 & 0.11 \\
\hline \multicolumn{5}{|c|}{$\mathbf{0 . 2 3 9} \mathbf{g} / \mathbf{s}, \mathbf{n}=\mathbf{8 4}$} \\
\hline Q1 & 0.039 & 0.018 & 0.17 & 0.070 \\
\hline Mean & 0.18 & 0.14 & 0.19 & 0.14 \\
\hline Median & 0.12 & 0.10 & 0.20 & 0.15 \\
\hline Q3 & 0.25 & 0.22 & 0.22 & 0.21 \\
\hline
\end{tabular}

The periods that contained no controlled release were also analyzed to test the machine learning models on the null dataset. There were 1208 15-minute periods that were analyzed with the OTM methods and the machine learning models. A box and whisker comparison of the non-controlled release periods are presented in Figure 47. The RMSE and the box plot statistics are presented in Table 67. 


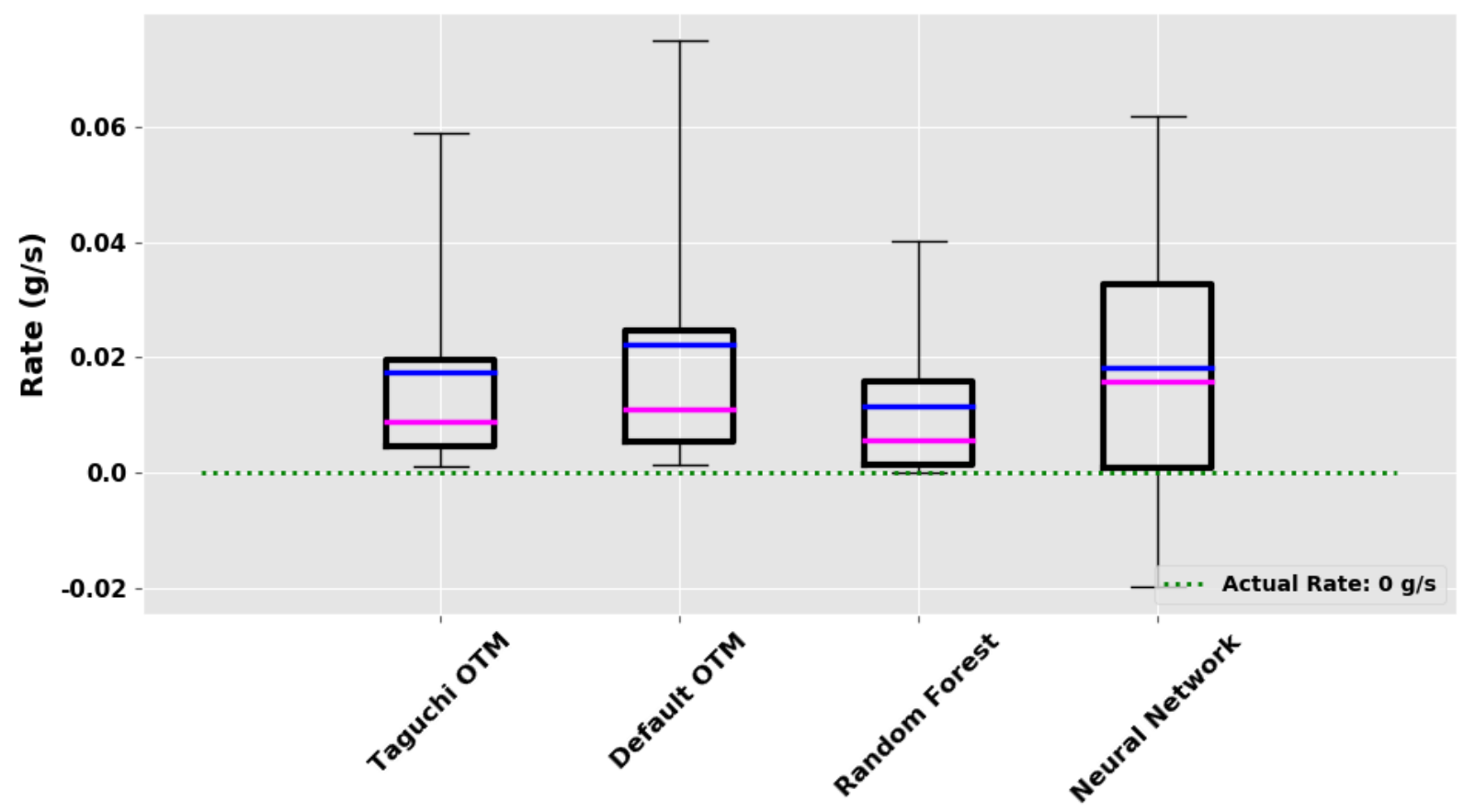

Figure 47. Box and whisker of default and Taguchi OTM, RF, and NN background estimates.

Table 67. Statistics of periods with no controlled release.

\begin{tabular}{|c|c|c|c|c|}
\hline Model & $\begin{array}{c}\text { Default } \\
\text { OTM }\end{array}$ & $\begin{array}{c}\text { Taguchi } \\
\text { OTM }\end{array}$ & RF & NN \\
\hline RMSE & 0.045 & 0.033 & 0.020 & 0.033 \\
\hline Q1 & 0.0053 & 0.0045 & 0.0014 & 0.0009 \\
\hline Mean & 0.022 & 0.018 & 0.012 & 0.018 \\
\hline Median & 0.011 & 0.0090 & 0.0056 & 0.016 \\
\hline Q3 & 0.025 & 0.020 & 0.016 & 0.033 \\
\hline
\end{tabular}

Historically OTM uncertainties have been quantified by using statistics of several measurements. The errors associated with the method have been presented as a range of error values as a percentage. To compare the uncertainties of these types of the entire controlled release dataset was analyzed by the default OTM, Taguchi OTM, RF and NN. Typically OTM estimations are eliminated if they produce a DQI value greater than 10 , which can significantly limit datasets, especially when data are collected continuously and no effort is made to collect data during optimal conditions. Of the 804 controlled release periods, accepted for analysis only $29 \%$ and $25 \%$, resulted in a DQI less than 10 for the default and Taguchi analyses, respectively. Part of the goal of this research was to develop methods that retained more data to help better understand the temporal variability of emissions. There will always be a tradeoff between the amount of data and the quality of data when dealing with the stochastic nature of micrometeorology. So while more data filtering could improve the accuracy of individual estimates, it also decreases the amount of data available for temporal analysis. For comparison to other OTM studies, the periods that produced a DQI value less than 10 for both the Taguchi and default OTM analysis $(n=181)$ were analyzed. The percent error distributions for the various controlled release rates are presented in Figure $48(n=43)$, Figure $49(n=99)$, 
and Figure $50(n=39)$. The data from the figures, and comparisons to previous controlled release studies are presented in Table 68. The RF and NN both improved upon the large datasets of the Taguchi and default OTM from this work. It is also noteworthy that they contained more periods within $\pm 50 \%$ than previous studies, suggesting that these methods may help to reduce the spread of predictions.

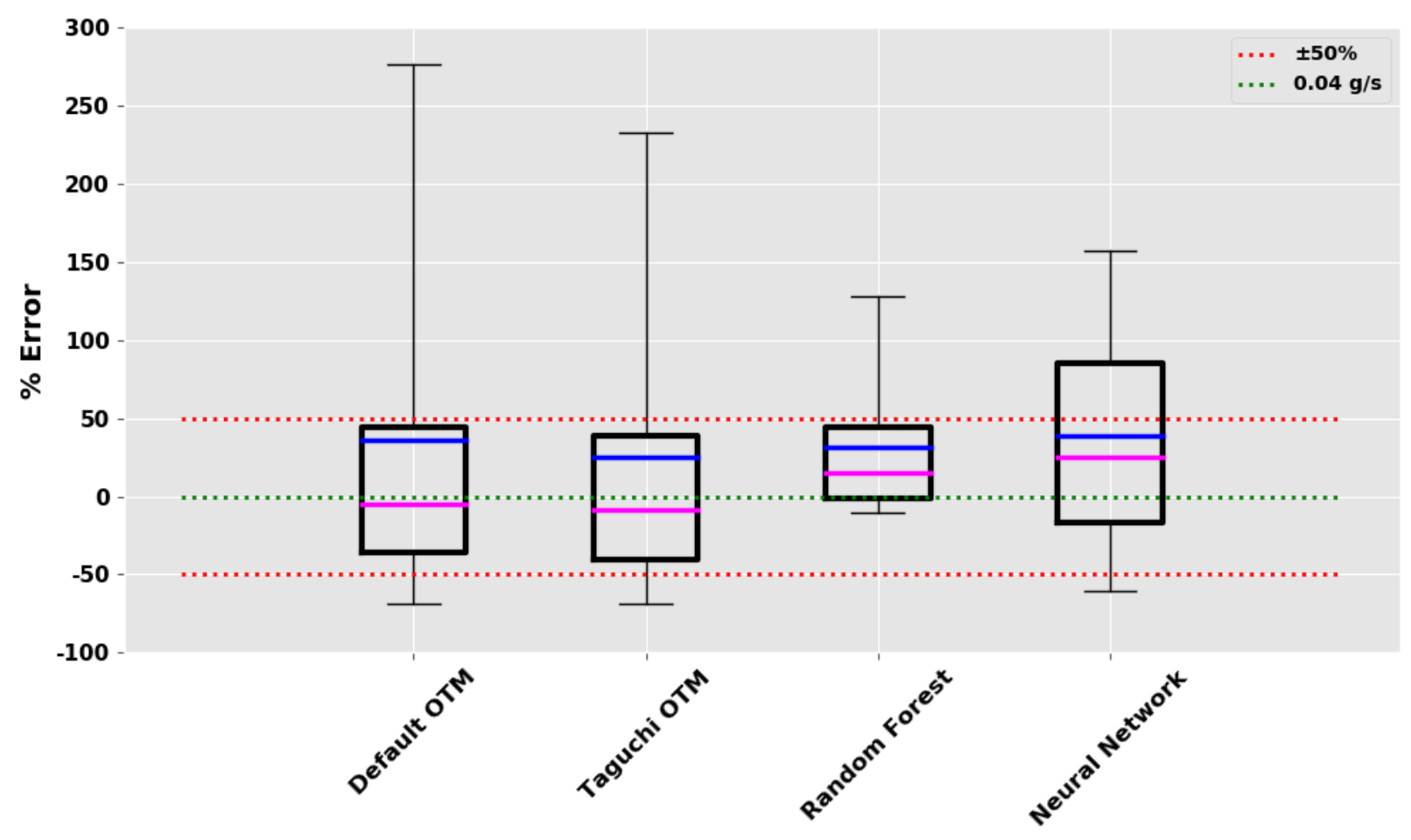

Figure 48. Low DQI percent error comparison for a controlled release rate of $0.04 \mathrm{~g} / \mathrm{s}$. 


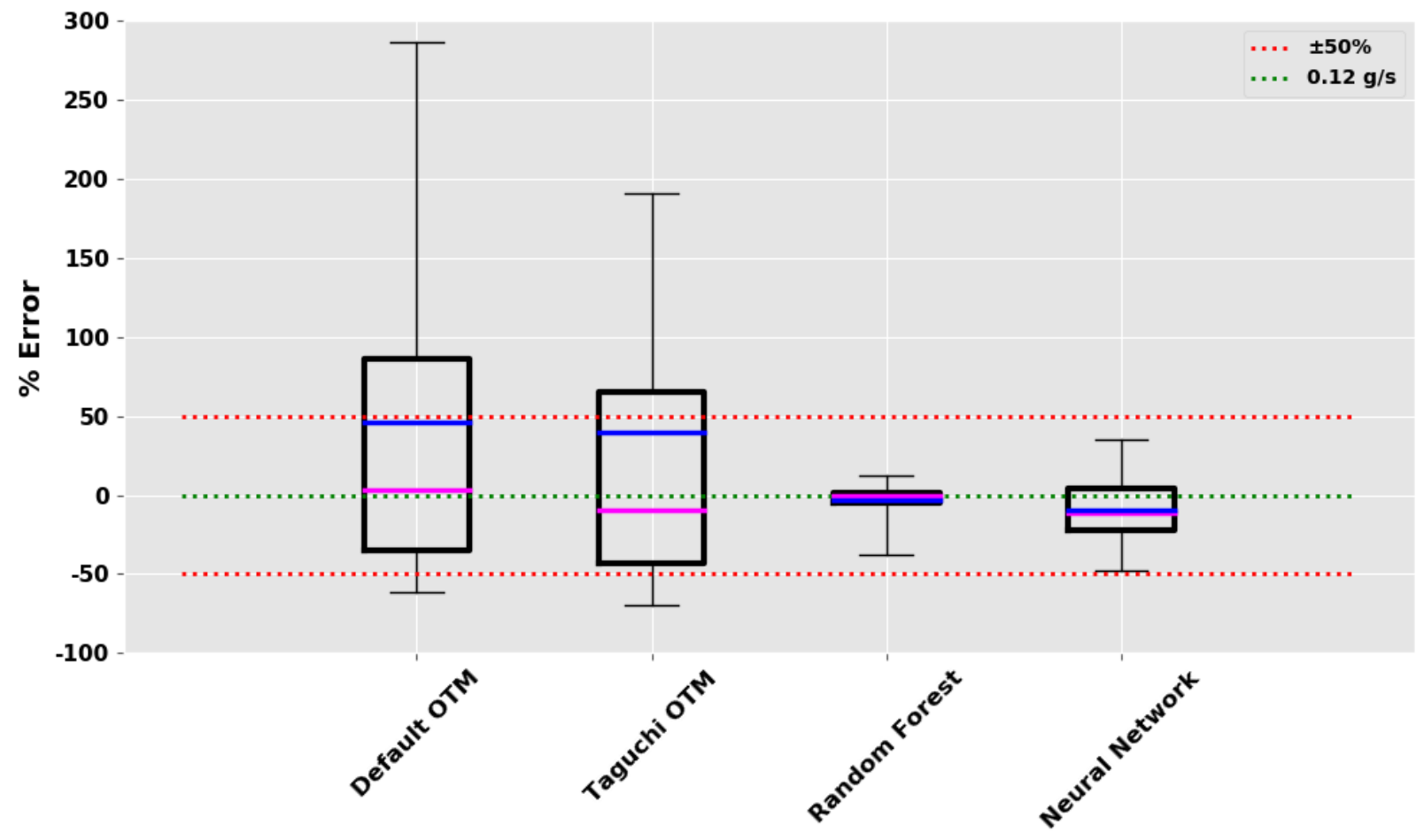

Figure 49. Low DQI percent error comparison for a controlled release rate of $0.12 \mathrm{~g} / \mathrm{s}$.

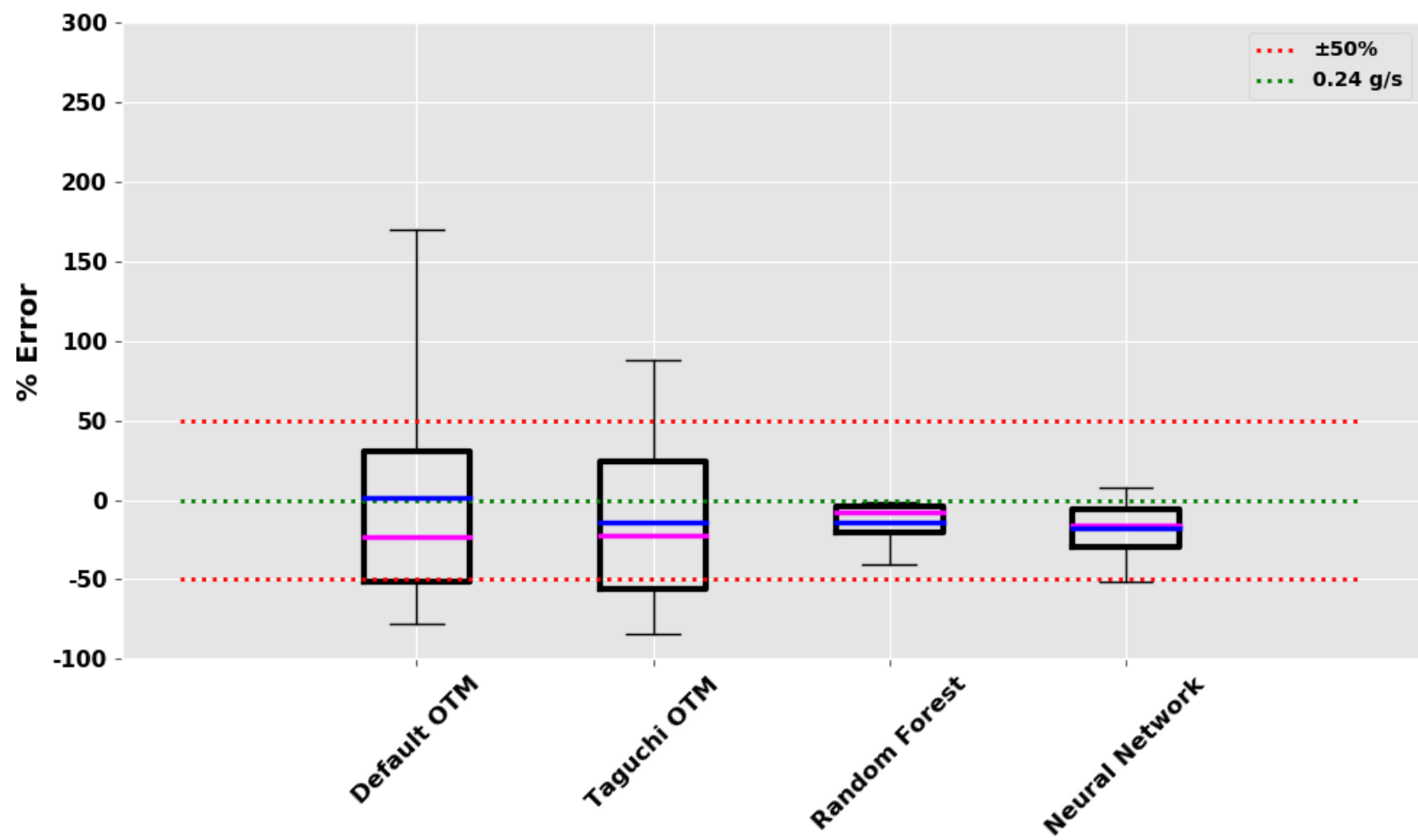

Figure 50. Low DQI percent error comparison for a controlled release rate of $0.24 \mathrm{~g} / \mathrm{s}$. 
Table 68. Comparison of results to previous OTM studies $(\mathrm{DQI}<10)$.

\begin{tabular}{|c|c|c|c|c|c|c|c|}
\hline & \multicolumn{3}{|c|}{ Previous Studies } & \multicolumn{4}{c|}{ This Work } \\
\cline { 2 - 8 } & $\begin{array}{c}\text { Robertson } \\
\text { et al. [51] }\end{array}$ & $\begin{array}{c}\text { Edie et } \\
\text { al. [53] }\end{array}$ & $\begin{array}{c}\text { Brantley } \\
\text { et al. [50] }\end{array}$ & $\begin{array}{c}\text { Default } \\
\text { OTM }\end{array}$ & $\begin{array}{c}\text { Taguchi } \\
\text { OTM }\end{array}$ & $\begin{array}{c}\text { Random } \\
\text { Forest }\end{array}$ & $\begin{array}{c}\text { Neural } \\
\text { Network }\end{array}$ \\
\hline Count (\#) & 19 & 24 & 107 & \multicolumn{4}{|c|}{181} \\
\hline $\begin{array}{c}\text { Release Rates } \\
\text { (g/s) }\end{array}$ & $0.03-0.56$ & $0.04-0.6$ & $0.19-1.2$ & \multicolumn{4}{c|}{$0.04-0.24$} \\
\hline $\begin{array}{c}\text { Full Range of } \\
\text { \% Error }\end{array}$ & $\begin{array}{c}-75 \% \text { to } \\
60 \%\end{array}$ & $\begin{array}{c}-60 \% \text { to } \\
175 \%\end{array}$ & $\begin{array}{c}-60 \% \text { to } \\
52 \%\end{array}$ & $\begin{array}{c}-95 \% \text { to } \\
1070 \%\end{array}$ & $\begin{array}{c}-95 \% \text { to } \\
1853 \%\end{array}$ & $\begin{array}{c}-75 \% \text { to } \\
186 \%\end{array}$ & $\begin{array}{c}-78 \% \text { to } \\
226 \%\end{array}$ \\
\hline $\begin{array}{c}\text { Tests within } \\
\pm \mathbf{3 0 \%}\end{array}$ & - & - & $71 \%$ & $30 \%$ & $31 \%$ & $85 \%$ & $65 \%$ \\
\hline $\begin{array}{c}\text { Tests within } \\
\pm 50 \%\end{array}$ & \multicolumn{2}{|c|}{$85 \%$} & - & $56 \%$ & $56 \%$ & $93 \%$ & $85 \%$ \\
\hline $\begin{array}{c}\mathbf{6 8 t h} \\
\text { Percentile } \\
\text { Error }\end{array}$ & $\pm 28 \%$ & $\pm 38 \%$ & - & $\pm 66 \%$ & $\pm 64 \%$ & $\pm 13 \%$ & $\pm 34 \%$ \\
\hline
\end{tabular}

\subsection{MSEEL Evaluations (Audits)}

The final models were compared to traditional methods on the data collected during MSEEL audits. All data collected within \pm 1 day of the audit were considered for these evaluations. It was acknowledged that this dataset was far different than the controlled release dataset. The MSEEL dataset had the potential for multiple leaks of various magnitudes from a variety of locations. All MSEEL components were within $20^{\circ}$ of each other with respect to the MECT location. The distances of the MSEEL component groups from the MECT varied between $54 \mathrm{~m}$ and $166 \mathrm{~m}$ as presented in Figure 21. While the nearest component group was well within the range of the distances tested on the controlled release experiment, the more distant components were at the edge of the feasibly measurable distance according to the traditional OTM suggestion of less than $200 \mathrm{~m}$ [47]. To compare model emissions predictions to audit measurements, the average distance $(121.8 \mathrm{~m})$ and direction $\left(167.5^{\circ}\right)$ to all potential sources were used. Due to tower positioning, it was difficult to distinguish emissions sources from one another. Also, according to Edie et al. the error associated with using an average distance to multiple sources was minimal compared to the overall error of the OTM method [53].

The valid MSEEL periods were identified in the same way as those from the controlled release experiments. In just over one year of data collection at the MSEEL site there were 35,364 periods which had the potential to produce an emissions estimate. Of these periods $71 \%$ produced valid EC and OTM results. Of the periods with successful EC and OTM calculations $41 \%$ were within $\pm 45^{\circ}$ of the mean direction of the sources. There were 1147 periods from days on which an audit took place. Of these 1147 periods, $56 \%$ produced valid EC and OTM estimates and $37 \%$ of those were within $\pm 45^{\circ}$ of the mean direction of the sources. A complete breakdown of the MSEEL periods is presented in Table 69. 
Table 69. Breakdown of available 15-minute periods from MSEEL.

\begin{tabular}{|l|c|c|}
\hline Period Attribute & All & Audit \\
\hline Total Possible & 35,364 & 1147 \\
\hline Valid EC and OTM & 25,256 & 670 \\
\hline $\pm 45^{\circ}$ Wind Direction (Valid Set) & 10,404 & 245 \\
\hline QC CH4 Flux $<2$ & 8018 & 184 \\
\hline OTM DQI $<10$ & 2314 & 28 \\
\hline Daytime (EddyPro) & 5774 & 170 \\
\hline
\end{tabular}

There were six direct quantification audits throughout the year the MECT was positioned on the MSEEL site. The emissions from the audits were presented in Table 24. The audit conducted during September of 2020 had a total site emissions rate of $0.1 \mathrm{~g} / \mathrm{s}$, however, there were no valid periods within 24 hours of the audit measurements and therefore it was not analyzed here. There was a total of 245 valid periods distributed among the remaining five audits.

Both the KM and FFP footprint estimations were applied to audit periods identified by the filters used on the controlled release data. There were zero periods on audit days in which either footprint method produced valid results. This was because the grid area identified by the mean distance and direction of the component groups on site never produced a footprint estimate of non-zero. An example of a period with no footprint contribution but with good wind direction is presented in Figure 52. Valid periods from the audit days were compared using multiple methods. The modeling (NN and RF) method estimates, and the Taguchi and default OTM estimates were compared across all audit days. The box and whisker plots of the data are presented in Figure 51 with statistics from the MECT audit set. The boxes encapsulate the lower and upper quartiles, the whiskers extend to the $5^{\text {th }}$ and $95^{\text {th }}$ percentiles, the blue dashed lines represent the means, and the red dotted lines represent the medians. Data outside of the $5^{\text {th }}$ and $95^{\text {th }}$ percentiles are not presented. The solid and dotted gray lines represent the weighted median and mean of the MECT audits, respectively. These values were calculated using all accepted periods within \pm 24 hours of an audit and assigning them the values of the audit. The standard deviation of the estimates from the Taguchi OTM were on average $24 \%$ less than those of the default OTM. The NN and RF reduced the standard deviation of estimates by $53 \%$ and $87 \%$, compared to the Taguchi OTM estimates. 
1.2

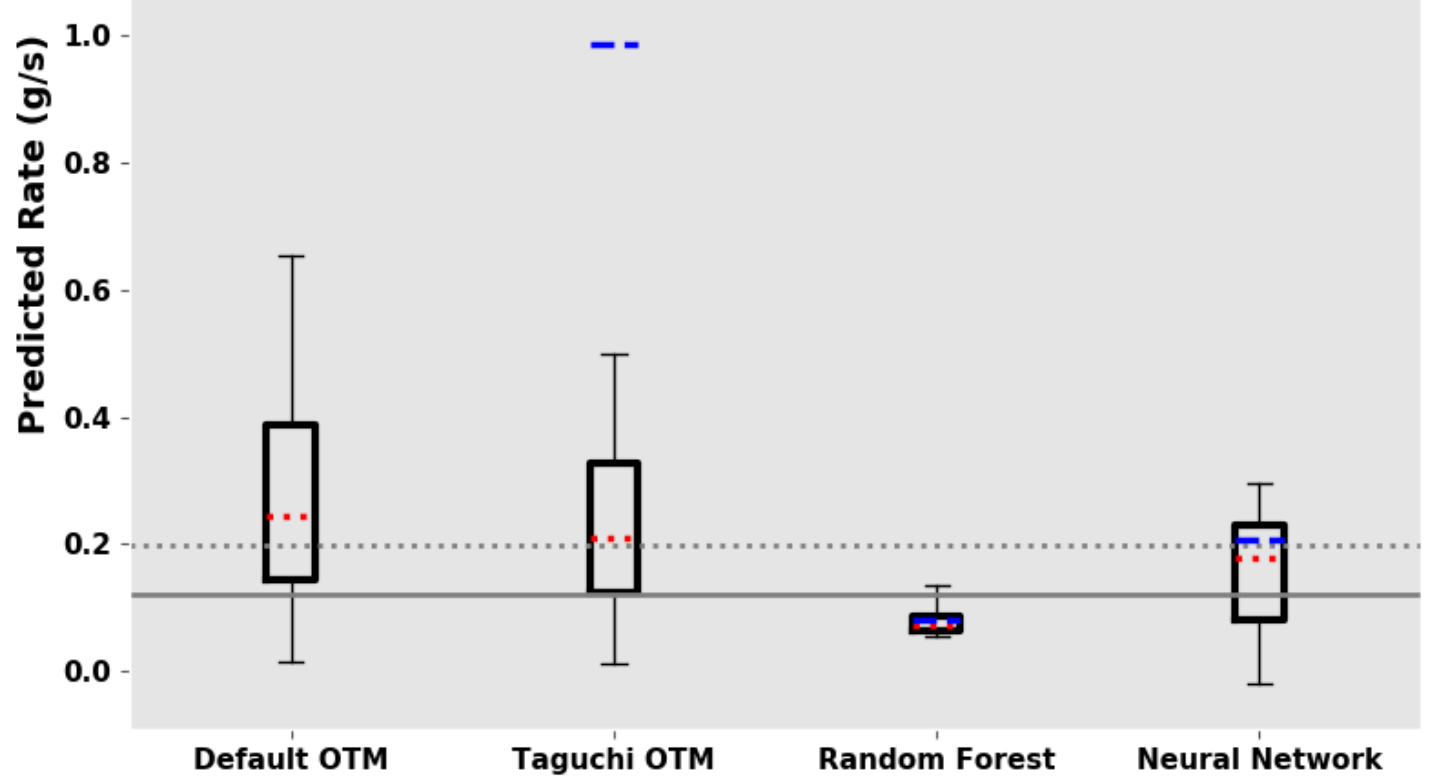

Figure 51. Comparison of default and Taguchi OTM, RF, and NN with MSEEL audits.

The RMSEs of the respective methods are presented in Table 70 for each individual audit. The Taguchi OTM RMSE was $24 \%$ less on average than the default OTM RMSE, across the five valid audits. The NN reduced the RMSE of 4 of the 5 estimates compared to the Taguchi OTM results. The NN performed poorly on the 2020-01 audit, causing an increase in RMSE of 187\% over the Taguchi OTM estimate. However, across all audits it reduced the RMSE by an average of 9\% and across the four audits that it did improve the average percent reduction was 58\%. The RF produced a lower RMSE than the Taguchi OTM estimate across all audits by an average of 49\%. The RF produced a lower RMSE than the NN on 3 of the 5 estimates.

Table 70. RMSEs of MSEEL audits for default and Taguchi OTM, RF, and NN.

\begin{tabular}{|c|c|c|c|c|c|c|}
\hline \multicolumn{3}{|c|}{ Information } & \multicolumn{4}{c|}{ RMSE (g/s) } \\
\hline $\begin{array}{c}\text { Audit } \\
\text { Date }\end{array}$ & $\begin{array}{c}\text { Site } \\
\text { Emissions } \\
(\mathbf{g} / \mathbf{s})\end{array}$ & $\begin{array}{c}\text { Count } \\
(\#)\end{array}$ & $\begin{array}{c}\text { Default } \\
\text { OTM }\end{array}$ & $\begin{array}{c}\text { Taguchi } \\
\text { OTM }\end{array}$ & $\begin{array}{c}\text { Random } \\
\text { Forest }\end{array}$ & $\begin{array}{c}\text { Neural } \\
\text { Network }\end{array}$ \\
\hline $2019-11$ & 0.17 & 78 & 0.13 & 0.11 & 0.10 & 0.071 \\
\hline $2020-01$ & 0.10 & 19 & 0.064 & 0.041 & 0.035 & 0.12 \\
\hline $2020-03$ & 0.67 & 32 & 0.91 & 0.86 & 0.59 & 0.51 \\
\hline $2020-06$ & 0.08 & 50 & 0.28 & 0.21 & 0.021 & 0.08 \\
\hline $2020-09$ & 0.10 & 0 & - & - & - & - \\
\hline $2020-11$ & 0.12 & 66 & 19.94 & 12.01 & 0.05 & 0.84 \\
\hline \multicolumn{2}{|c|}{ All } & 245 & 10.36 & 6.24 & 0.22 & 0.48 \\
\hline
\end{tabular}



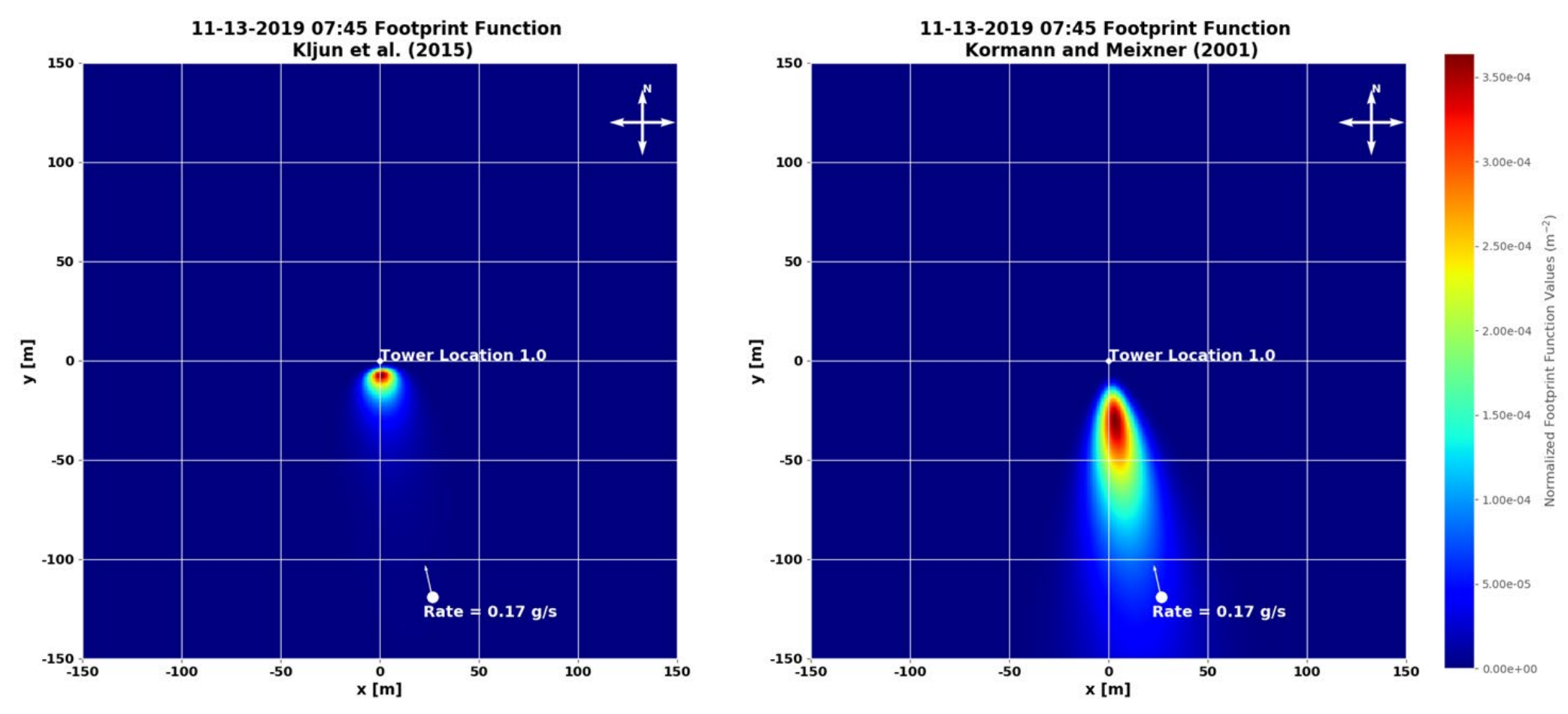

Figure 52. MSEEL footprint heatmap of an example 15-minute period. 


\subsection{MSEEL Predictions (Non-Audits)}

The Taguchi OTM, NN, and RF estimates were evaluated for valid periods of data collected during the entire MSEEL deployment. There were 10,159 valid 15-minute periods within the WF over the course of the collection period, excluding periods within 24 hours of an audit. These periods were used as inputs to the different methods to produce MSEEL site level emissions estimates. The average distance to component groups and StS direction were again used for all estimates. While these estimates were not validated against a known mass emission rate, they provide insights into the efficacy of the various methods. For comparison, the distributions and statistics of these predictions were compared to all 17 MSEEL audits, which were presented in Table 24. The statistics of the estimates from the three methods and those of the direct quantification audits are presented in Table 71. It should be noted that the distribution of all 17 audits is not necessarily representative of the emissions during the data collection period, which occurred between November 2019 and December 2020. This is shown by the last two columns in Table 71 where "All" and "MECT" represent the statistics of all the audits, and those with the MECT deployed, respectively.

The audit emissions measurements are heavily skewed due to "super-emitter" type events during the October 2018 and the December 2018 audits. As previously discussed, emissions from production sites sometimes experience "super-emitter" tendencies. Zavala-Araiza et al. used $26 \mathrm{~kg} / \mathrm{hr}$ as a "super-emitter" threshold [25]. Emissions during the October 2018 audit would have represented a "super-emitter" condition by this definition. Caulton et al. defined "super-emitters" as sites with emissions rates greater than $9 \mathrm{~kg} / \mathrm{hr}$ [35]. The two highest emitting audits would be classified as "super-emitters" with this metric. Because of these statistics, the entire distribution of audit measurements may not be representative of those during MECT deployment.

As previously discussed, the distribution of emissions from NG production sites has a fat-tailed shape. The Taguchi OTM over-estimated the maximum emission rate of all audits by an order of magnitude. It also tended to over-estimate at the lower end of the distribution. The NN predicted a maximum that was only $2 \%$ different than that of the maximum audit. The $\mathrm{NN}$ also better replicated emissions on the low end of the predictions. However, the geometric mean of the NN predictions was $53 \%$ less than that of all audits. Both the RF and NN were more similar to the MECT audits across all metrics than the Taguchi OTM estimates. However, the RF predictions were not believed to be very representative of the MSEEL site. The problem with the RF was that it was unable to extrapolate beyond its training set targets. This is a fallback of RFs in general and is well documented. The maximum prediction of the RF was an order of magnitude smaller than the maximum audit measurement. It underpredicted emissions compared to audits on all statistical measurements, when compared to the full set and MECT audits.

The box and whisker plots of the data are presented in Figure 53 with statistics from the full audit set and the MECT audit set. The boxes encapsulate the lower and upper quartiles, the whiskers extend to the $5^{\text {th }}$ and $95^{\text {th }}$ percentiles, the blue dashed lines represent the means, and the red dotted lines represent the medians. Data outside of the $5^{\text {th }}$ and $95^{\text {th }}$ percentiles are not presented. The black and gray solid lines represent the medians of all the audits and the MECT audits, respectively. The black and gray dotted lines represent the means of all the audits and the MECT audits, respectively.

Distributions of the data are presented in Figure 54, where blue bars represent the distribution of the given method and the red bars represent the distribution of the audits. The x-axis shows the upper limit of the histogram bins. The averages of the highest bins $(>1)$ of the audits, Taguchi OTM estimates, and NN estimates were 5.8, 8.7, and $3.2 \mathrm{~g} / \mathrm{s}$, respectively. There were no RF predictions greater than $1 \mathrm{~g} / \mathrm{s}$. 
Table 71. Statistics of MSEEL predictions and audits.

\begin{tabular}{|l|c|c|c|c|c|}
\hline \multicolumn{1}{|c|}{ Metric / Method } & $\begin{array}{c}\text { Taguchi } \\
\text { OTM }\end{array}$ & RF & NN & $\begin{array}{c}\text { Audits } \\
\text { (All) }\end{array}$ & $\begin{array}{c}\text { Audits } \\
\text { (MECT) }\end{array}$ \\
\hline Count (\#) & 10,159 & 10,159 & 10,159 & 17 & 6 \\
\hline Mean (g/s) & 0.58 & 0.10 & 0.20 & 1.18 & 0.21 \\
\hline Standard Deviation (g/s) & 3.59 & 0.04 & 0.32 & 2.98 & 0.23 \\
\hline Minimum (g/s) & 0.01 & 0.00 & 0.00 & 0.02 & 0.08 \\
\hline 25th Percentile (g/s) & 0.16 & 0.07 & 0.10 & 0.10 & 0.10 \\
\hline 50th Percentile (g/s) & 0.24 & 0.09 & 0.17 & 0.13 & 0.11 \\
\hline 75th Percentile (g/s) & 0.36 & 0.13 & 0.25 & 0.49 & 0.16 \\
\hline Maximum (g/s) & 124 & 0.23 & 12 & 12 & 0.67 \\
\hline Geometric Mean (g/s) & 0.24 & 0.10 & 0.11 & 0.23 & 0.15 \\
\hline
\end{tabular}

1.2

1.0 -
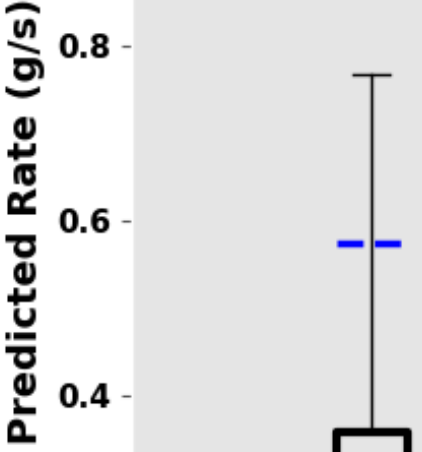

All Audit Median

.... All Audit Mean MECT Audit Median MECT Audit Mean

0.2

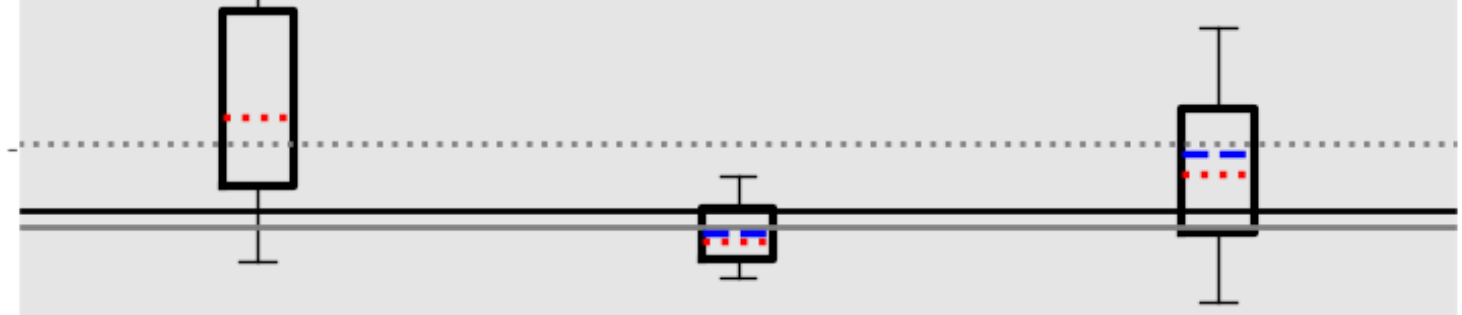

0.0

Taguchi OTM

Random Forest

Neural Network

Figure 53. Box and whisker of OTM, RF, and NN predictions compared to MSEEL audit statistics. 

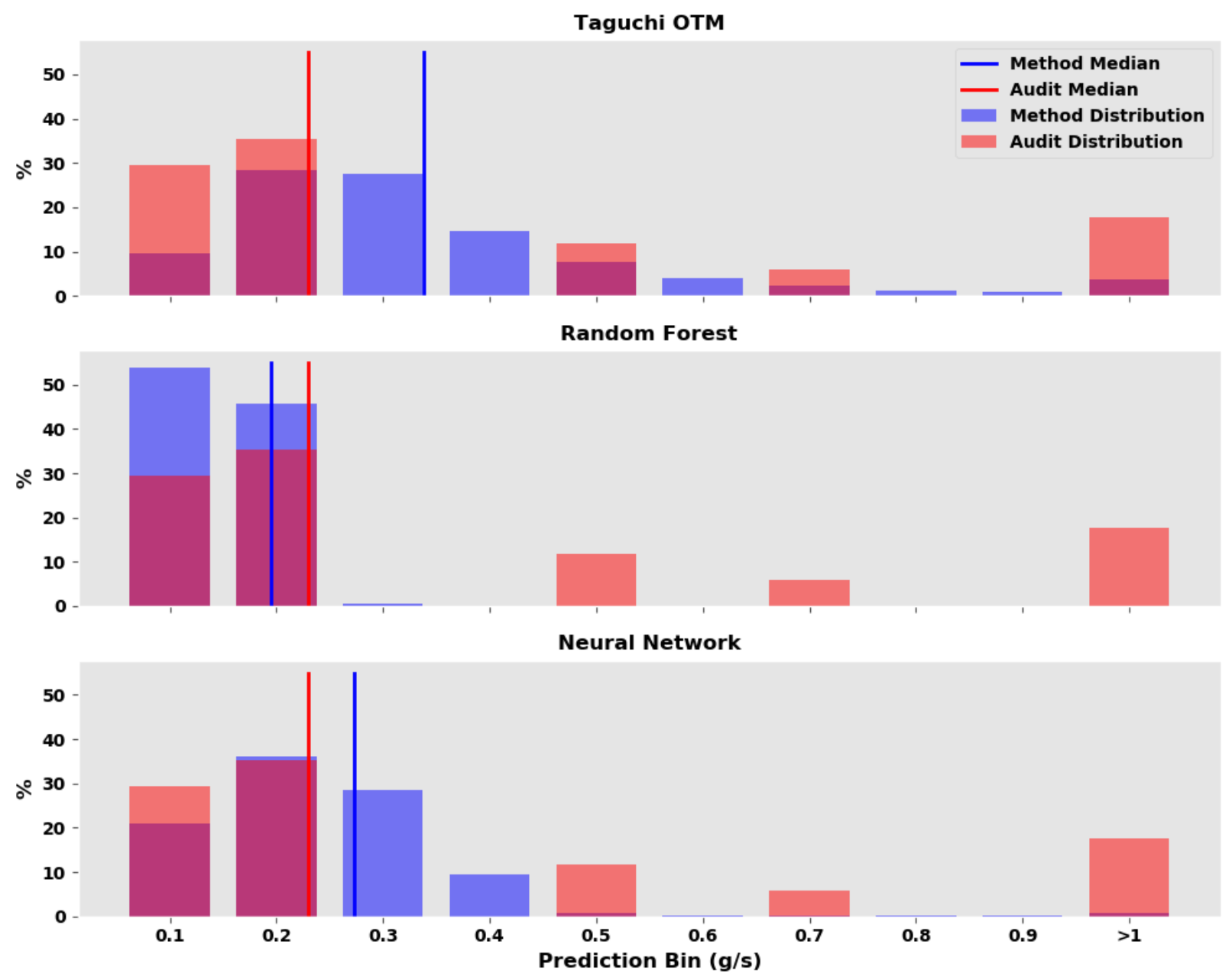

Figure 54. Distributions of methods overlayed with audit measurements.

This analysis made evident the RF's inability to extrapolate beyond the scope of training data. This lack of extrapolation was the methods largest downfall. The $\mathrm{NN}$ was able to extrapolate predicted emissions rates but its predictions were not as large as those made by Taguchi OTM alone. Figure 55 presents the daily average predictions of the methods with two different scales. The top graph is scaled by the maximum prediction of the NN. The bottom graph is scaled by the maximum controlled release rate tested at the farm, and in turn the maximum prediction of the RF. It should be noted that there were many Taguchi OTM predictions greater than the maximum NN prediction, as presented in Figure 55. The black and gray solid lines represent the medians of all the audits and the MECT audits, respectively. The black and gray dotted lines represent the means of all the audits and the MECT audits, respectively. 

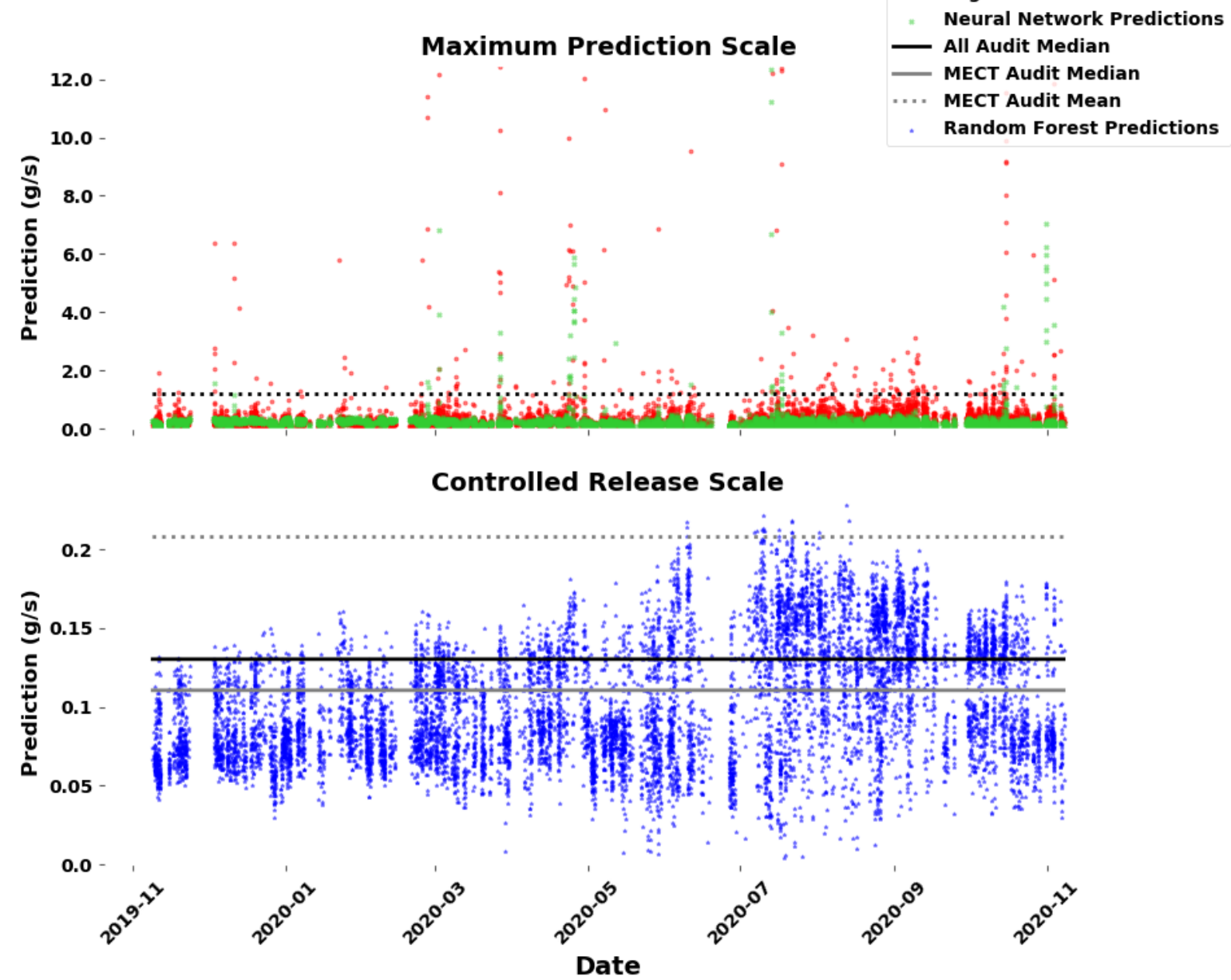

Figure 55. Predictions of MSEEL emission from various methods.

\section{Conclusions}

Current methods of stationary single sensor indirect quantification are limited by their high uncertainties and the stochastic nature of micrometeorological modeling. The objectives defined by a recent NSF grant to WVU focused on the "inter-comparison and advancement" of direct and indirect methane emissions measurements. The objectives were to directly quantify emissions, develop and deploy a DAQ system to record variables required for currently used indirect quantification techniques, and utilize machine learning to combine current methods to reduce indirect quantification uncertainty. To accomplish these objectives, the OTM and EC techniques for quantifying methane fluxes were analyzed.

The first methodology, OTM, has historically been used to quantify methane emissions from NG infrastructure sites. It relies on a PSG method to estimate horizontal fluxes in the near field (less than 200 $\mathrm{m}$ downwind). There was no literature at the time of this writing on attempts to improve or refine the technique, although some researchers have used methods that rely on the same principles. To improve the accuracy of OTM, binning parameters were optimized with the use of a Taguchi design array. The goal was to improve OTM measurements without discarding a significant percentage of measurements due to failed calculations. The Taguchi OTM parameters were able to improve the RMSE of a random sample of measurements made during a series of controlled releases of methane. The Taguchi OTM estimates resulted in a lower RMSE across all RRD scenarios except for one $(0.119 \mathrm{~g} / \mathrm{s}, 72 \mathrm{~m})$. The Taguchi OTM RMSE of 
this scenario was $2 \%$ higher than the default OTM results. However, the Taguchi OTM RMSE was on average 22\% lower across all scenarios. The Taguchi OTM results also reduced the standard deviation of measurements for the same RRD scenarios. The average standard deviation of the RRD scenario estimates was reduced by $30 \%$ using the optimized parameters. These results suggest that the method on average produced less error and was more repeatable.

Previous research efforts have reported on the measurement error of OTM by comparing multiple estimates to known releases under favorable conditions. They have bounded their CIs by the distribution of errors from these tests and have assumed $2 \sigma$ errors of $\pm 50 \%$ to $\pm 70 \%$. There is limited published research on the repeatability of the method or the baseline measurement error. Here I have presented a methodology to estimate the baseline measurement error due to instrumentation and atmospheric stochasticity. Using stateof-the-art DAQ instrumentation, the average uncertainty due to measurement limitations was determined to be $\pm 3.8 \%$ of the reported measurement. This was determined with a non-parametric bootstrap of randomly selected averaging periods collected during controlled release experiments. Only further improvement in instrument technology could resolve such errors. A second measure of uncertainty meant to quantify the uncertainty associated with stochastic measurements was developed by H\&R for estimating EC uncertainty. The method attempts to quantify the randomness of measurements by comparing similar periods exactly 24-hours apart that are expected to produce the same estimates. This method was utilized to estimate the OTM uncertainty due to the randomness of micrometeorological variables even when the most important conditions are similar. Such uncertainty will always be present in real-world measurements due to unrepeatability. The uncertainty from this method on average was $\pm 17 \%$ of the reported measurement across all measured distances. The combination of these two uncertainties results in a minimum obtainable uncertainty of $\pm 17.4 \%$ for an individual measurement. This uncertainty will always be present in field measurements when using the OTM method. This is less than previously reported $1 \sigma$ errors of $\pm 28 \%$ and $\pm 38 \%$. However, these uncertainties are based on the statistics of a population of comparisons between estimates and known rates with low DQI (less than 10) and favorable conditions. The uncertainty presented here is the uncertainty associated with the measurement itself (i.e., the estimated value should be considered to have error bars of $\pm 17 \%$ ), so this is the absolute minimum uncertainty attainable, and would never truly be achieved. To compare to the uncertainties of previously reported studies, periods with a DQI less than $10(\mathrm{n}=181)$ were analyzed. The $1 \sigma$ error of the OTM method with the default parameters was $\pm 66 \%$. The Taguchi optimization improved these results to $\pm 64 \%$. These $1 \sigma$ errors are closer to the $2 \sigma$ errors previously reported. The reason for the discrepancy is likely the bigger datasets, which are more likely to contain large outliers. OTM tends to over-estimate small rates (less than $1 \mathrm{~kg} / \mathrm{hr}$ or $\sim 0.3 \mathrm{~g} / \mathrm{s}$ ) and therefore can produce erroneous results when attempting to quantify similar rates, especially at longer $(>100 \mathrm{~m})$ distances.

The second methodology, EC, is much more established in the research community and is widely accepted as a means of quantifying vertical fluxes from homogeneous area sources. The literature on anthropogenic point sources is much more limited. Here I have analyzed established methods of estimating the uncertainty of EC results and applied them to a series of measurements made with a single controlled release present. The average uncertainties of the EC method at the Reedsville Farm site were $\pm 35 \%$ and $\pm 42 \%$ from the F\&S and H\&R methods, respectively. The F\&S method did not account for the uncertainty in the flux footprint whereas the H\&R method does. To estimate a mass rate from the EC method, some knowledge of the source area was required. The contribution to the total flux from the source area was estimated with various footprint models, which have been utilized by previous research. Two widely used footprint models were applied to the EC results to quantify the mass emissions of the known, point source releases with little success. Both the KM and FFP models significantly underestimated mass emissions. Across 142 periods that produced valid methane mass estimates, the KM and FFP predictions were on average $61 \%$ and $83 \%$ less than the actual rate.

To estimate point source emissions from individual averaging periods, EC footprint and OTM methods have been analyzed from a variety of perspectives. Of 142 periods that produced valid results from both estimates the EC FFP and KM footprint estimates resulted in MAPEs of 84\% and 83\%, respectively. The 
Taguchi OTM resulted in an MAPE of 44\%. However, both methods not only have high errors associated with repeat measurements, but also large uncertainties associated with individual measurements. Due to the stochastic nature of such measurements, it is unlikely that such uncertainties will be overcome with a single sensor measurement method.

However, as this research showed, it is possible to make both measurements simultaneously. The EC and OTM DAQ instrumentation setups have significant overlap and state-of-the-art equipment is sufficient for the measurements required of both methods. To take advantage of this, a multi-method stationary single sensor approach was used. The approach relied on a MECT, which was developed for the purpose of recording the variables required for both traditional processing techniques. This approach used the raw data and processing required for both methods individually. Their respective outputs were then used as inputs for machine learning algorithms. This methodology showed improvement compared to the traditional methods' individual estimates. Variables utilized and produced by the EC and OTM methods were combined and analyzed with a variety of techniques. Two machine learning methods stood out as promising ways to increase the accuracy of in-field emissions measurements. A RF and MLP NN offered robustness and accuracy.

The RF and NN models were trained on a subset of the controlled release dataset and used to predict the mass emissions. Model inputs were selected based on correlations with known release rates, correlations, with other features, and model improvement and robustness. Model improvements were quantified by a reduction in the RMSE of a test dataset. The final feature set of the models consistent of 18 variables produced by the traditional methods. Variables from traditional methods were used as the goal was to develop models which could enhance current techniques rather than bypass them. Model hyper-parameters were optimized using random searches with cross-validation. Optimized feature selection and hyperparameters reduced the RMSE produced by the RF and NN by $4 \%$ and $40 \%$, respectively. The models were compared to traditional methods across several datasets.

The EC with two footprint methods, Taguchi OTM, RF, and NN results were compared on the periods with valid footprint estimates $(n=142)$. This allowed for direct comparisons of all method estimates of methane mass emissions rates. The NN RMSE of this dataset was 54\% and 37\% lower than the KM footprint and Taguchi OTM RMSEs, respectively. The RF showed even more improvement with an RMSE 50\% lower than the NN. This dataset was limited by the restrictions of the EC footprint calculation requirements. To avoid elimination of large amounts of data the footprint estimates were not required model inputs.

The RF, NN, and default and Taguchi OTM models were also compared across a dataset of periods with DQI values less than $10(n=181)$. Previous studies involving OTM have typically discarded measurements with a DQI above 10 . The $1 \sigma$ errors of the default and Taguchi OTM from this dataset were $\pm 66 \%$ and $\pm 64 \%$, respectively. The NN and RF produced $1 \sigma$ errors of $\pm 34 \%$ and $\pm 13 \%$, respectively. They also reduced the range of estimates. Across the three different controlled release rates the NN and RF reduced the standard deviation of estimates by an average of $63 \%$ and $74 \%$, respectively, compared to the Taguchi OTM estimates.

Both traditional methods significantly limited the amount of data available. Neither a valid footprint estimate nor a low DQI were required for data to be utilized by the RF or NN. However, the DQI and other variables related to footprints were used as model inputs. This was believed to increase the number of available periods while simultaneously allowing models to compensate for lower quality periods. The RF and $\mathrm{NN}$ models were therefore able to produce estimates across the entire release dataset $(\mathrm{n}=804)$. Traditional and Taguchi OTM values were compared to these estimates, although based on historical OTM research, many of these periods would have been discarded. Across the three release rates of the dataset the Taguchi OTM RMSE was on average 27\% lower than the default OTM RMSE. The NN and RF further reduced the RMSE by an average of $44 \%$ and $69 \%$, respectively. Both the NN and RF models also produced estimates closer to zero during periods where no controlled release was present, compared to OTM results. 
To expand beyond the controlled releases, models were utilized at the MSEEL site for long-term emissions estimations. Direct quantification audits were used as a point of comparison for both traditional methods and models. The direct quantification audits produce an accurate $( \pm 10 \%)$ estimate of emissions from the site for the given period. The EC footprint methods produced no valid estimates based on the average distance to the components on the site. The Taguchi OTM estimates reduced the RMSE of the audit estimates by $40 \%$ compared to the default OTM estimates. The NN and RF produced RMSEs that were $92 \%$ and $96 \%$ lower than the Taguchi OTM, respectively. The NN and RF reduced the standard deviation of audit estimates by an average of $53 \%$ and $87 \%$, respectively, compared to Taguchi OTM results.

Across the full year of predictions, Taguchi OTM estimates had a higher variance than either the NN or the $\mathrm{RF}$. When comparing the results of these methods, however, it is important to note that the NN was far more robust than the $\mathrm{RF}$, in its ability to extrapolate beyond the test set. This indicated that the similarity between the RF's statistics and the audit results during MECT deployment could be misleading due to the relatively low emissions of those specific audits. The mean NN mass emission rate prediction was only $6 \%$ less than the mean emission rate of the audits conducted during MECT deployment, and the standard deviation was only $39 \%$ greater. While this distribution was similar, the $\mathrm{NN}$ also indicated a few instances of emissions rates that were of the same order of magnitude as the highest rates seen during the "superemitter" audits. This may suggest that higher levels of emissions did occur which the audits did not have capture due to temporal variability. While findings here were difficult to quantify, they indicate that the combined methods could produce more reliable results than long term OTM or EC measurements alone. Such estimates could aid in scaling emissions geospatially from site to regional or national levels and temporally from the minute/hour to yearly scales.

In summary, some key quantifiable findings were:

- $\quad$ Taguchi OTM reduced RMSE across all RRD scenarios by an average of $22 \%$

- Taguchi OTM reduced the standard deviation across RRD scenarios by an average of $30 \%$

- Minimum attainable measurement uncertainty of the OTM method was determined to be $\pm 17 \%$ based on instrument limitations and H\&R for the RRD scenarios

- Minimum attainable measurement uncertainty of the EC method to estimate mass emissions was determined to be $\pm 42 \%$ based on the H\&R method for the RRD scenarios

- $\quad \mathrm{KM}$ and FFP footprint methods rarely produced mass emissions estimates for distances greater than $50 \mathrm{~m}$ and underestimated by an average of $61 \%$ and $83 \%$, respectively

- $\quad$ RF and NN models were developed and optimized, reducing baseline RMSEs by $4 \%$ and $40 \%$, respectively

- $\quad$ The optimized models reduced the RMSE of all datasets compared to the EC footprint and default and Taguchi OTM measurements

- The NN and RF reduced the standard deviation of estimates with a DQI less than 10 by an average of $63 \%$ and $74 \%$, respectively, compared to Taguchi OTM estimates

- Across the full release dataset, the NN and RF RMSEs were 44\% and 69\% lower than Taguchi OTM

- Average Taguchi OTM RMSE and standard deviation were 24\% lower than default OTM values across MSEEL direct quantification audits

- $\quad$ RF and NN RMSEs were 49\% and 9\% lower on average than the Taguchi OTM estimates across direct quantification audits

\section{Further Discussions}

Here, methods for the indirect quantification of methane emissions have been evaluated. The traditional methods for estimating mass emissions from stationary single sensors are poorly constrained and have high uncertainties. This research demonstrated that methods for improving estimates was possible through both traditional methods (OTM) or a combined (EC and OTM) approach with machine learning algorithms (RF 
or NN). However, neither of the machine learning algorithms were optimized at the conclusion of this research. The limited dataset utilized here allowed for comparison to a small number of controlled releases and five direct quantification audits at a real-world NG production site. The efficacy of either of these techniques as a realistic solution for improving the accuracy of measurements depends on several factors.

The main drawback of the RF was its inability to extrapolate beyond its initial training scope. This is a critical flaw in the method when moving from a controlled experiment to a real-world site. However, this could be overcome with a wider range of controlled release rates, distances, and site geometries. The RF could then be trained on a more robust dataset which in turn would allow the method to make better predictions in real-world scenarios. The problem with such an approach is that it is a slippery slope. Where does one stop expanding a controlled release matrix that is meant to encapsulate any real-world scenario? Even with this downfall, the RF could still provide value and insight into emissions predictions. The RF could be combined with other methods such as a NN or linear regressor. In such a scenario, the RF would be relied upon when the predicted emissions were below a pre-defined threshold based on its training and a factor of safety. Say that the RF was trained on release rates up to $0.24 \mathrm{~g} / \mathrm{s}$, as was performed here. The algorithm could say that if the prediction from the RF was above $80 \%$ of this threshold, then an alternative algorithm which allows extrapolation should be used. The case in this research where this is most applicable was the MSEEL full year predictions, where the NN appeared to perform better above the $50^{\text {th }}$ percentile of audit estimates. If a RF were not used for any predictions of mass emissions estimates, its ability to identify key features could still be utilized. This would be valuable when combining with any type of NN as they are notoriously difficult to interpret and have the stigma of being a "black box" methodology. The use of a RF to identify features which could be used as inputs to a robust NN could be applied to any dataset, as it was in this work. As more unique scenarios and more data were obtained, the importance of various features could be altered as the $\mathrm{NN}$ evolved from more training iterations.

A key drawback of using NNs is the requirement that the features be scaled or normalized. This makes recursive training difficult because the model cannot simply be "updated" with new data. Instead, if a MLP was used, as it was here, the entire potential dataset would have to be rescaled and the NN retrained. This could be overcome by initially testing what are believed to be the distributions of each variable in a controlled release dataset. The difficulty of such as task would depend on the features believed to be required for inputs. Suggested limits could be easily defined for some variables such as temperature, air heat capacity, signal strength, and StS direction. Such variables have natural bounds. However, it would be much more difficult to set the bounds of variables such as methane flux, OTM estimates, or methane variance. The limits of these variables would likely not only depend on the ranges of mass emissions rates, but also on-site geometries, tower placement, and surrounding ecological conditions. These factors combined with the stochasticity of micrometeorological measurements, would make recursive training without rescaling a near impossibility. In addition, if the standard scaler were used, as in this work, the distributions of such variables would also be required. This data would likely be unknown no matter the scenario. These factors point towards the data requiring rescaling when the set is expanded.

It could be asked whether the current methodologies are required at all. One of the goals of the research was to combine current methods with machine learning to reduce indirect quantification uncertainty. For this reason, traditional methods were first used to calculate results, which were then utilized as inputs to machine learning. This would allow for historical measurements and those reported from current data collection to be utilized with the methods developed. For example, historical data sets from EC towers may have more than enough data to produce Taguchi OTM results that could then be combined with the existing data using methods described herein.

However, alternative input datasets could be developed from the raw data collected. These data could be raw measured variables, averages, or period distributions. Many machine learning methods, especially NNs can handle various types of inputs. NNs are well equipped to handle pattern recognition problems and could be well suited to make predictions of emissions rates based on different input datasets. While this would 
eliminate the use of current methods, it may allow for better predictions as the input data could be more detailed. This may "paint a better picture" which the algorithm could then identify. Such a process could make use of a convolutional NN, which are often used for image recognition. While this was not the goal of this work, a raw data matrix could act as an "image" for a given period. If the NN were trained in this way, each period would be input as a data matrix of raw time versus each of the collected variables. One downfall already discussed could be the scaling of such variables. Utilizing raw data may require more preprocessing. Future research could explore the use of raw or averaged data as an input which could help to remove the uncertainties associated traditional methods. This could allow the NN or other machine learning algorithm to become more robust as it would not be limited by fallbacks of current technique calculations.

With such drawbacks or alternatives in mind, the machine learning models utilized in this work could overcome significant obstacles with more concurrent direct measurements. Most models can be improved with higher quality input data. As measurements were performed in the field, the size and scope of the available data would continue to grow. Models trained on datasets of the highest quality would become more robust in their ability to interpret complex scenarios. The training and testing sets used in this research were limited to a small series of controlled releases. Ideally training sets could be expanded in the following ways:

1. More release rates - expanding the controlled release rate limits would expand the bounds of both models.

2. Different site geometries - better representation of NG infrastructure sites with the potential for multiple leaks would be of use.

3. Varied atmospheric conditions - changing the time of year of the training data and ensuring that the same releases were performed under various conditions would eliminate reliance on unrealistic correlations, which could be a source of error in the models.

One way to rapidly increase the training set would be to deploy the MECT during LDAR programs that were coupled with quantification efforts. Even if the MECT was only on a site for a day during a LDAR and quantification audit, it would add valuable training data to models. In such a scenario, during a direct quantification campaign the MECT would be positioned on site at the start of the audit. Observation of wind direction would help with positioning of the MECT. Once deployed, a range finder or GPS coordinates could be used to estimate distances and StS directions. While the data would be limited if only deployed for a single day, over the course of a series of campaigns the dataset would expand and ideally encapsulate a wide range of scenarios on which the model could be trained. The MECT could then be deployed at similar sites, long-term, to allow for better understanding of the temporal variability of emissions.

Scenarios could exist where it would be advantageous to position the MECT in the middle of multiple potential sources. This would decrease distances to the sources, which could increase accuracy. It would also allow for multiple individual sources to be quantified with changing wind direction throughout the campaign. For example, in this research, only the average distance to site components was utilized for OTM and EC footprint calculations, however, if individual components were analyzed separately within a model, more accurate predictions may be attainable. If a site operator sought more knowledge about individual sources, tower placement relative to target sources could provide valuable insight. This could make identification of background sources more difficult. To overcome this, different definitions of accepted periods could be utilized. For example, in this analysis only controlled releases with distances less than 50 $\mathrm{m}$ provided a footprint estimate for EC methods. By requiring such footprint estimates (even if not accurate) as a feature, components could be more easily identified and separated. Rather than a large amount of data being eliminated by a WF, different wind directions would represent different components or groups of components on the site in question. In this work, the positioning of the MECT was limited to where the site operator allowed, which happened to be far from any potential sources. However, this would not always be 
the case. Such methods could also help to quantify sources that are usually inaccessible for safety reasons, which could help to enhance LDAR and quantification programs.

Ultimately, methods that rely on micrometeorological measurements and a single-point sensor will always be associated with relatively high uncertainties compared to direct measurements or more robust multisensor measurements. However, steps can be taken to enhance current methods, which may result in fewer discrepancies between inventories and research campaigns. Much of the recently funded research focused on methane emissions quantification has investigated completely new measurement technologies ranging from drones to satellites to complex optical sensors. These technologies are meant to produce either more frequent measurements or cover a wider area with more precision. Even with such technologies complex algorithms are often required to produce semi-accurate results. Combining such algorithms and technologies is not an easy task for industry. The novel methodology suggested here is applicable with technology and measurement methods already in use by research and industry. The data processing methods are already available. It is believed that with more collected data and the combination of these methods with the simple and effective machine learning algorithms presented here that the methods would become more robust and accurate.

Better understanding of the temporal distribution of emissions could help target reductions. Improvements in long-term indirect measurements that are low cost will help researchers and industry understand the problem of "super-emitters" as well. It may turn out that sites are only "super-emitters" intermittently, which would drastically change their emissions profiles compared to simply assuming that emissions are constant. Elucidating a clearer picture of emissions profiles both temporally and geo-spatially will drastically improve our understanding of the NG methane problem. While this work has not solved the problem explicitly, the research has identified methods that could enhance OTM alone, by reducing variability and increasing average accuracy. By using data from multiple approaches and with the inclusion of machine learning methods, the work performed here could provide a pathway to a better understanding of emissions. The research is also applicable with currently available state-of-the-art instruments and would not require extensive sensor counts or computational resources. 


\section{References}

[1] "DOE Announces $\$ 13$ Million to Quantify and Mitigate Methane Emissions from Natural Gas Infrastructure," Energy.gov. https://www.energy.gov/articles/doe-announces-13-million-quantifyand-mitigate-methane-emissions-natural-gas-infrastructure (accessed Mar. 08, 2021).

[2] "Methane Detectors Challenge," Environmental Defense Fund. https://www.edf.org/methanedetectors-challenge (accessed Mar. 08, 2021).

[3] "Methane research series: 16 studies," Environmental Defense Fund. https://www.edf.org/climate/methane-research-series-16-studies (accessed Mar. 08, 2021).

[4] "MONITOR | arpa-e.energy.gov." https://arpa-e.energy.gov/technologies/programs/monitor (accessed Mar. 08, 2021).

[5] "NSF Award Search: Award\#1804024 - Inter-comparison of Direct Quantification and Areal Micrometeorological Methods to Investigate the Transport and Fate of Methane from Heterogeneous Sources in Natural Gas Fields.” https://www.nsf.gov/awardsearch/showAward?AWD_ID=1804024\&HistoricalAwards=false (accessed Mar. 08, 2021).

[6] G. Myhre et al., "8 Anthropogenic and Natural Radiative Forcing," in Climate Change 2013: The Physical Science Handbook, Cambridge University Press, 2013, p. 82. [Online]. Available: http://www.climatechange2013.org/images/report/WG1AR5_Chapter08_FINAL.pdf

[7] O. US EPA, "Overview of Greenhouse Gases," US EPA, Dec. 23, 2015. https://www.epa.gov/ghgemissions/overview-greenhouse-gases (accessed Jan. 02, 2020).

[8] O. US EPA, "Natural Gas and Petroleum Systems in the GHG Inventory: Additional Information on the 1990-2018 GHG Inventory (published April 2020)," US EPA, Apr. 09, 2020. https:/www.epa.gov/ghgemissions/natural-gas-and-petroleum-systems-ghg-inventory-additionalinformation-1990-2018-ghg (accessed Mar. 08, 2021).

[9] N. US Department of Commerce, "Global Monitoring Laboratory - Carbon Cycle Greenhouse Gases." https://www.esrl.noaa.gov/gmd/ccgg/trends_ch4/(accessed Mar. 08, 2021).

[10] "Climate Change Indicators: Atmospheric Concentrations of Greenhouse Gases | Climate Change Indicators in the United States | US EPA." https://climatechange.chicago.gov/climateindicators/climate-change-indicators-atmospheric-concentrations-greenhouse-gases (accessed Apr. 22, 2021).

[11] O. US EPA, "Overview of Greenhouse Gases," US EPA, Dec. 23, 2015. https://www.epa.gov/ghgemissions/overview-greenhouse-gases (accessed Jan. 02, 2020).

[12] O. US EPA, "Importance of Methane," US EPA, Jan. 11, 2016. https://www.epa.gov/gmi/importancemethane (accessed Jan. 02, 2020).

[13] S. M. Miller et al., "Anthropogenic emissions of methane in the United States," Proceedings of the National Academy of Sciences, vol. 110, no. 50, pp. 20018-20022, Dec. 2013, doi: 10.1073/pnas.1314392110.

[14] "Methane from oil \& gas - Methane Tracker 2020 - Analysis," IEA. https://www.iea.org/reports/methane-tracker-2020/methane-from-oil-gas (accessed Mar. 08, 2021).

[15] "Fast Facts." Environmental Protection Agency, Apr. 2020. Accessed: Mar. 08, 2021. [Online]. Available: https://www.epa.gov/sites/production/files/2020-04/documents/fastfacts-1990-2018.pdf

[16] "Supersize Me! Marcellus/Utica Well Pads Now Host Up to 40 Wells," Marcellus Drilling News, Jan. 16, 2018. https://marcellusdrilling.com/2018/01/supersize-me-marcellus-utica-well-pads-nowhost-up-to-40-wells/ (accessed Jan. 02, 2020).

[17] "These days, oil and gas companies are super-sizing their well pads | Pittsburgh Post-Gazette." https://www.post-gazette.com/business/powersource/2018/01/15/These-days-oil-and-gascompanies-are-super-sizing-their-well-pads/stories/201801140023 (accessed Jan. 02, 2020).

[18] J. Snyder, R. Oommen, and M. Pring, "Oil and Gas 101: An Overview of Oil and Gas Upstream Activities and Using EPA's Nonpoint Oil and Gas Emission Estimation Tool for the 2014 NEI," presented at the Emission Inventory Conference, San Diego, CA, Apr. 14, 2015. Accessed: Jan. 03, 
2020. [Online]. Available: https://www.epa.gov/sites/production/files/201509/documents/101_part_1.pdf

[19] M. Omara, M. R. Sullivan, X. Li, R. Subramanian, A. L. Robinson, and A. A. Presto, "Methane Emissions from Conventional and Unconventional Natural Gas Production Sites in the Marcellus Shale Basin," Environ. Sci. Technol., vol. 50, no. 4, pp. 2099-2107, Feb. 2016, doi: 10.1021/acs.est.5b05503.

[20] O. US EPA, "Estimates of Methane Emissions by Segment in the United States," US EPA, Aug. 27, 2018. https://www.epa.gov/natural-gas-star-program/estimates-methane-emissions-segment-unitedstates (accessed Mar. 08, 2021).

[21] "Number of Producing Gas Wells.” https://www.eia.gov/dnav/ng/ng_prod_wells_s1_a.htm (accessed Jan. 02, 2020).

[22] "United States - Maps - U.S. Energy Information Administration (EIA)." https://www.eia.gov/state/maps.php?v=Natural\%20Gas (accessed Mar. 08, 2021).

[23] "EIA expects natural gas production and exports to continue increasing in most scenarios - Today in Energy - U.S. Energy Information Administration (EIA)." https://www.eia.gov/todayinenergy/detail.php?id=42875 (accessed Mar. 08, 2021).

[24] "Inventory of U.S. Greenhouse Gas Emissions and Sinks," U.S. Environmental Protection Agency, 430-R-19-001, Apr. 2019. [Online]. Available: https://www.epa.gov/sites/production/files/201904/documents/us-ghg-inventory-2019-main-text.pdf

[25] D. Zavala-Araiza et al., "Toward a Functional Definition of Methane Super-Emitters: Application to Natural Gas Production Sites," Environ. Sci. Technol., vol. 49, no. 13, pp. 8167-8174, Jul. 2015, doi: 10.1021/acs.est.5b00133.

[26] M. Omara et al., "Methane Emissions from Natural Gas Production Sites in the United States: Data Synthesis and National Estimate," Environ. Sci. Technol., vol. 52, no. 21, pp. 12915-12925, Nov. 2018, doi: 10.1021/acs.est.8b03535.

[27] B. Luck et al., "Multiday Measurements of Pneumatic Controller Emissions Reveal the Frequency of Abnormal Emissions Behavior at Natural Gas Gathering Stations," Environ. Sci. Technol. Lett., vol. 6, no. 6, pp. 348-352, Jun. 2019, doi: 10.1021/acs.estlett.9b00158.

[28] T. L. Vaughn et al., "Temporal variability largely explains top-down/bottom-up difference in methane emission estimates from a natural gas production region," Proc Natl Acad Sci USA, vol. 115, no. 46, pp. 11712-11717, Nov. 2018, doi: 10.1073/pnas.1805687115.

[29] D. Johnson, R. Heltzel, and D. Oliver, "Temporal Variations in Methane Emissions from an Unconventional Well Site," ACS Omega, vol. 4, no. 2, pp. 3708-3715, Feb. 2019, doi: 10.1021/acsomega.8b03246.

[30] R. W. Howarth, R. Santoro, and A. Ingraffea, "Methane and the greenhouse-gas footprint of natural gas from shale formations: A letter," Climatic Change, vol. 106, no. 4, pp. 679-690, Jun. 2011, doi: 10.1007/s10584-011-0061-5.

[31] R. A. Alvarez, S. W. Pacala, J. J. Winebrake, W. L. Chameides, and S. P. Hamburg, "Greater focus needed on methane leakage from natural gas infrastructure," Proceedings of the National Academy of Sciences, vol. 109, no. 17, pp. 6435-6440, Apr. 2012, doi: 10.1073/pnas.1202407109.

[32] A. R. Brandt et al., "Methane Leaks from North American Natural Gas Systems," Science, vol. 343, no. 6172, pp. 733-735, Feb. 2014, doi: 10.1126/science.1247045.

[33] D. T. Allen et al., "Measurements of methane emissions at natural gas production sites in the United States," Proceedings of the National Academy of Sciences, vol. 110, no. 44, pp. 17768-17773, Oct. 2013, doi: 10.1073/pnas.1304880110.

[34] D. Zavala-Araiza et al., "Reconciling divergent estimates of oil and gas methane emissions," Proc Natl Acad Sci USA, p. 201522126, Dec. 2015, doi: 10.1073/pnas.1522126112.

[35] D. R. Caulton et al., "Importance of Superemitter Natural Gas Well Pads in the Marcellus Shale," Environ. Sci. Technol., vol. 53, no. 9, pp. 4747-4754, May 2019, doi: 10.1021/acs.est.8b06965.

[36] CFR, "e-CFR Title 40 Part $60 \rightarrow$ Subpart OOOO," eCFR.io. https://ECFR.io/Title-40/sp40.8.60.oooo (accessed Mar. 13, 2020). 
[37] O. US EPA, "Method 21 - Volatile Organic Compound Leaks," US EPA, Jun. 21, 2016. https://www.epa.gov/emc/method-21-volatile-organic-compound-leaks (accessed Mar. 13, 2020).

[38] D. E. Kimbrel, J. Balasbas, J. Zolnierek, J. Fallah, and C. Thibodeaux, "Sampling of Methane Emissions Detection Technologies and Practices for Natural Gas Distribution Infrastructure," Natural Gas Infrastructure Modernization Partnership, 2019.

[39] D. T. Allen et al., "Methane Emissions from Process Equipment at Natural Gas Production Sites in the United States: Pneumatic Controllers," Environ. Sci. Technol., vol. 49, no. 1, pp. 633-640, Jan. 2015, doi: 10.1021/es5040156.

[40] A. P. Pacsi et al., "Equipment leak detection and quantification at 67 oil and gas sites in the Western United States," Elem Sci Anth, vol. 7, no. 1, p. 29, Jul. 2019, doi: 10.1525/elementa.368.

[41] J. Kuo, T. C. Hicks, B. Drake, and T. F. Chan, "Estimation of methane emission from California natural gas industry," Journal of the Air \& Waste Management Association, vol. 65, no. 7, pp. 844855, Jul. 2015, doi: 10.1080/10962247.2015.1025924.

[42] M. Kang et al., "Direct measurements of methane emissions from abandoned oil and gas wells in Pennsylvania," Proc Natl Acad Sci USA, vol. 111, no. 51, pp. 18173-18177, Dec. 2014, doi: 10.1073/pnas.1408315111.

[43] T. Howard, T. W. Ferrara, and A. Townsend-Small, "Sensor transition failure in the high flow sampler: Implications for methane emission inventories of natural gas infrastructure," Journal of the Air \& Waste Management Association, vol. 65, no. 7, pp. 856-862, Jul. 2015, doi: 10.1080/10962247.2015.1025925.

[44] J. I. Connolly, R. A. Robinson, and T. D. Gardiner, "Assessment of the Bacharach Hi Flow® Sampler characteristics and potential failure modes when measuring methane emissions," Measurement, vol. 145, pp. 226-233, Oct. 2019, doi: 10.1016/j.measurement.2019.05.055.

[45] T. Howard, "University of Texas study underestimates national methane emissions at natural gas production sites due to instrument sensor failure," Energy Sci Eng, vol. 3, no. 5, pp. 443-455, Sep. 2015, doi: 10.1002/ese3.81.

[46] Clearstone Engineering, "Methane Emission Measurement Techniques," presented at the Methane to Markets Partnership, Buenos Aires, Argentina, Nov. 05, 2018.

[47] E. D. Thoma, H. Brantley, B. Squier, J. DeWees, R. Segall, and R. Merrill, "Development of Mobile Measurement Method Series," p. 15.

[48] “OTM 33A V1.3." Environmental Protection Agency, Nov. 01, 2014. [Online]. Available: https://www3.epa.gov/ttnemc01/prelim/otm33a.pdf

[49] O. US EPA, "EMC Other Test Methods," US EPA, Jun. 17, 2016. https://www.epa.gov/emc/emcother-test-methods (accessed Jan. 02, 2020).

[50] H. L. Brantley, E. D. Thoma, W. C. Squier, B. B. Guven, and D. Lyon, "Assessment of Methane Emissions from Oil and Gas Production Pads using Mobile Measurements," Environ. Sci. Technol., vol. 48, no. 24, pp. 14508-14515, Dec. 2014, doi: 10.1021/es503070q.

[51] A. M. Robertson et al., "Variation in Methane Emission Rates from Well Pads in Four Oil and Gas Basins with Contrasting Production Volumes and Compositions," Environ. Sci. Technol., vol. 51, no. 15, pp. 8832-8840, Aug. 2017, doi: 10.1021/acs.est.7b00571.

[52] A. M. Robertson et al., "New Mexico Permian Basin Measured Well Pad Methane Emissions Are a Factor of 5-9 Times Higher Than U.S. EPA Estimates," Environ. Sci. Technol., p. acs.est.0c02927, Oct. 2020, doi: 10.1021/acs.est.0c02927.

[53] R. Edie et al., "Constraining the accuracy of flux estimates using OTM 33A," Atmos. Meas. Tech., vol. 13, no. 1, pp. 341-353, Jan. 2020, doi: 10.5194/amt-13-341-2020.

[54] D. A. Snare, "A Comparison of Ground-Based and Aircraft-Based Methane Emission Flux Estimates in a Western Oil and Natural Gas Production Basin," MS, University of Wyoming, Laramie, Wyoming, 2015.

[55] C. S. Bell et al., "Comparison of methane emission estimates from multiple measurement techniques at natural gas production pads," Elem Sci Anth, vol. 5, no. 0, p. 79, Dec. 2017, doi: 10.1525/elementa.266. 
[56] John. D. Albertson et al., "A Mobile Sensing Approach for Regional Surveillance of Fugitive Methane Emissions in Oil and Gas Production," Environ. Sci. Technol., vol. 50, no. 5, pp. 2487-2497, Mar. 2016, doi: 10.1021/acs.est.5b05059.

[57] X. Zhou et al., "Mobile sampling of methane emissions from natural gas well pads in California," Atmospheric Environment, vol. 244, p. 117930, Jan. 2021, doi: 10.1016/j.atmosenv.2020.117930.

[58] T. I. Yacovitch et al., "Mobile Laboratory Observations of Methane Emissions in the Barnett Shale Region," Environ. Sci. Technol., vol. 49, no. 13, pp. 7889-7895, Jul. 2015, doi: 10.1021/es506352j.

[59] J. D. Goetz et al., "Atmospheric Emission Characterization of Marcellus Shale Natural Gas Development Sites," Environ. Sci. Technol., vol. 49, no. 11, pp. 7012-7020, Jun. 2015, doi: 10.1021/acs.est.5b00452.

[60] C. W. Rella, T. R. Tsai, C. G. Botkin, E. R. Crosson, and D. Steele, "Measuring Emissions from Oil and Natural Gas Well Pads Using the Mobile Flux Plane Technique," Environ. Sci. Technol., vol. 49, no. 7, pp. 4742-4748, Apr. 2015, doi: 10.1021/acs.est.5b00099.

[61] A. Karion, C. Sweeney, P. Tans, and T. Newberger, "AirCore: An Innovative Atmospheric Sampling System," J. Atmos. Oceanic Technol., vol. 27, no. 11, pp. 1839-1853, Nov. 2010, doi: 10.1175/2010JTECHA1448.1.

[62] X. Lan, R. Talbot, P. Laine, and A. Torres, "Characterizing Fugitive Methane Emissions in the Barnett Shale Area Using a Mobile Laboratory," Environ. Sci. Technol., vol. 49, no. 13, pp. 8139-8146, Jul. 2015, doi: 10.1021/es5063055.

[63] P. E. Saide et al., "Evaluating Methods To Estimate Methane Emissions from Oil and Gas Production Facilities Using LES Simulations," Environ. Sci. Technol., vol. 52, no. 19, pp. 11206-11214, Oct. 2018, doi: 10.1021/acs.est.8b01767.

[64] G. Burba, Eddy covariance method for scientific, industrial, agricultural, and regulatory applications: a field book on measuring ecosystem gas exchange and areal emission rates. Lincoln, Nebraska: LI-COR Biosciences, 2013.

[65] "AmeriFlux Management Project," Earth and Environmental Sciences Area. https://eesa.lbl.gov/projects/ameriflux-management-project/ (accessed Jan. 10, 2020).

[66] P. C. Stoy, A. A. Cook, J. E. Dore, W. Kleindl, and E. N. J. Brookshire, "Methane efflux from an American bison herd," p. 30, 2020.

[67] P. Dumortier, M. Aubinet, F. Lebeau, A. Naiken, and B. Heinesch, "Point source emission estimation using eddy covariance: Validation using an artificial source experiment," Agricultural and Forest Meteorology, vol. 266-267, pp. 148-156, Mar. 2019, doi: 10.1016/j.agrformet.2018.12.012.

[68] T. W. Coates, T. K. Flesch, S. M. McGinn, E. Charmley, and D. Chen, "Evaluating an eddy covariance technique to estimate point-source emissions and its potential application to grazing cattle," Agricultural and Forest Meteorology, vol. 234-235, pp. 164-171, Mar. 2017, doi: 10.1016/j.agrformet.2016.12.026.

[69] A. C. I. Tang et al., "Eddy Covariance Measurements of Methane Flux at a Tropical Peat Forest in Sarawak, Malaysian Borneo," Geophys. Res. Lett., vol. 45, no. 9, pp. 4390-4399, May 2018, doi: 10.1029/2017GL076457.

[70] C. A. Emmerton, V. L. St. Louis, I. Lehnherr, E. R. Humphreys, E. Rydz, and H. R. Kosolofski, "The net exchange of methane with high Arctic landscapes during the summer growing season," Biogeosciences, vol. 11, no. 12, pp. 3095-3106, Jun. 2014, doi: 10.5194/bg-11-3095-2014.

[71] P. Prajapati and E. A. Santos, "Measurements of methane emissions from a beef cattle feedlot using the eddy covariance technique," Agricultural and Forest Meteorology, vol. 232, pp. 349-358, Jan. 2017, doi: 10.1016/j.agrformet.2016.09.001.

[72] P. Prajapati and E. A. Santos, "Estimating methane emissions from beef cattle in a feedlot using the eddy covariance technique and footprint analysis," Agricultural and Forest Meteorology, vol. 258, pp. 18-28, Aug. 2018, doi: 10.1016/j.agrformet.2017.08.004.

[73] "AmeriFlux - Overview-Year of Methane," AmeriFlux. https://ameriflux.lbl.gov/year-ofmethane/year-of-methane/ (accessed Mar. 08, 2021). 
[74] C.-I. Hsieh, G. Katul, and T. Chi, "An approximate analytical model for footprint estimation of scalar fluxes in thermally stratifed atmospheric flows," Advances in Water Resources, p. 8, 2000.

[75] R. Kormann and F. X. Meixner, "An Analytical Footprint Model For Non-Neutral Stratification," Boundary-Layer Meteorology, vol. 99, no. 2, pp. 207-224, May 2001, doi: 10.1023/A:1018991015119.

[76] N. Kljun, P. Calanca, M. W. Rotach, and H. P. Schmid, "A simple two-dimensional parameterisation for Flux Footprint Prediction (FFP)," Geosci. Model Dev., vol. 8, no. 11, pp. 3695-3713, Nov. 2015, doi: 10.5194/gmd-8-3695-2015.

[77] N. Kljun, P. Calanca, M. W. Rotach, and H. P. Schmid, "A Simple Parameterisation for Flux Footprint Predictions," Boundary-Layer Meteorology, vol. 112, no. 3, pp. 503-523, Sep. 2004, doi: 10.1023/B:BOUN.0000030653.71031.96.

[78] T. Flesch, J. Wilson, L. Harper, and B. Crenna, "Estimating gas emissions from a farm with an inverse-dispersion technique," Atmospheric Environment, vol. 39, no. 27, pp. 4863-4874, Sep. 2005, doi: 10.1016/j.atmosenv.2005.04.032.

[79] T. W. Horst, "On Frequency Response Corrections for Eddy Covariance Flux Measurements," Boundary-Layer Meteorology, vol. 94, no. 3, pp. 517-520, Mar. 2000, doi: 10.1023/A:1002427517744.

[80] K. Heidbach, H. P. Schmid, and M. Mauder, "Experimental evaluation of flux footprint models," Agricultural and Forest Meteorology, vol. 246, pp. 142-153, Nov. 2017, doi: 10.1016/j.agrformet.2017.06.008.

[81] J. D. Wilson, "Computing the Flux Footprint," Boundary-Layer Meteorol, vol. 156, no. 1, pp. 1-14, Jul. 2015, doi: 10.1007/s10546-015-0017-9.

[82] D. Y. Hollinger and A. D. Richardson, "Uncertainty in eddy covariance measurements and its application to physiological models," Tree Physiology, vol. 25, no. 7, pp. 873-885, Jul. 2005, doi: 10.1093/treephys/25.7.873.

[83] P. L. Finkelstein and P. F. Sims, "Sampling error in eddy correlation flux measurements," J. Geophys. Res., vol. 106, no. D4, pp. 3503-3509, Feb. 2001, doi: 10.1029/2000JD900731.

[84] J. D. Wilson and E. Yee, "A critical examination of the random displacement model of turbulent dispersion," Boundary-Layer Meteorol, vol. 125, no. 3, pp. 399-416, Oct. 2007, doi: 10.1007/s10546007-9201-x.

[85] T. Foken, M. Göockede, M. Mauder, L. Mahrt, B. Amiro, and W. Munger, "Post-Field Data Quality Control," in Handbook of Micrometeorology, vol. 29, X. Lee, W. Massman, and B. Law, Eds. Dordrecht: Kluwer Academic Publishers, 2005, pp. 181-208. doi: 10.1007/1-4020-2265-4_9.

[86] M. Detto and G. G. Katul, "Simplified expressions for adjusting higher-order turbulent statistics obtained from open path gas analyzers," Boundary-Layer Meteorol, vol. 122, no. 1, pp. 205-216, Jan. 2007, doi: 10.1007/s10546-006-9105-1.

[87] M. J. Deventer et al., "Error characterization of methane fluxes and budgets derived from a long-term comparison of open- and closed-path eddy covariance systems," Agricultural and Forest Meteorology, vol. 278, p. 107638, Nov. 2019, doi: 10.1016/j.agrformet.2019.107638.

[88] A. Karion et al., "Methane emissions estimate from airborne measurements over a western United States natural gas field," Geophys. Res. Lett., vol. 40, no. 16, pp. 4393-4397, Aug. 2013, doi: $10.1002 /$ grl.50811.

[89] M. L. Smith, E. A. Kort, A. Karion, C. Sweeney, S. C. Herndon, and T. I. Yacovitch, "Airborne Ethane Observations in the Barnett Shale: Quantification of Ethane Flux and Attribution of Methane Emissions," Environ. Sci. Technol., vol. 49, no. 13, pp. 8158-8166, Jul. 2015, doi: 10.1021/acs.est.5b00219.

[90] M. L. Smith et al., "Airborne Quantification of Methane Emissions over the Four Corners Region," Environ. Sci. Technol., vol. 51, no. 10, pp. 5832-5837, May 2017, doi: 10.1021/acs.est.6b06107.

[91] T. N. Lavoie et al., "Aircraft-Based Measurements of Point Source Methane Emissions in the Barnett Shale Basin," Environ. Sci. Technol., vol. 49, no. 13, pp. 7904-7913, Jul. 2015, doi: 10.1021/acs.est.5b00410. 
[92] J. Peischl et al., "Quantifying atmospheric methane emissions from the Haynesville, Fayetteville, and northeastern Marcellus shale gas production regions: CH4 emissions from shale gas production," $J$. Geophys. Res. Atmos., vol. 120, no. 5, pp. 2119-2139, Mar. 2015, doi: 10.1002/2014JD022697.

[93] S. Schwietzke et al., "Improved Mechanistic Understanding of Natural Gas Methane Emissions from Spatially Resolved Aircraft Measurements," Environ. Sci. Technol., vol. 51, no. 12, pp. 7286-7294, Jun. 2017, doi: 10.1021/acs.est.7b01810.

[94] G. Pétron et al., "A new look at methane and nonmethane hydrocarbon emissions from oil and natural gas operations in the Colorado Denver-Julesburg Basin: Hydrocarbon emissions in oil \& gas basin," J. Geophys. Res. Atmos., vol. 119, no. 11, pp. 6836-6852, Jun. 2014, doi: 10.1002/2013JD021272.

[95] A. Karion et al., "Aircraft-Based Estimate of Total Methane Emissions from the Barnett Shale Region," Environ. Sci. Technol., vol. 49, no. 13, pp. 8124-8131, Jul. 2015, doi: 10.1021/acs.est.5b00217.

[96] D. R. Caulton et al., "Toward a better understanding and quantification of methane emissions from shale gas development," Proceedings of the National Academy of Sciences, vol. 111, no. 17, pp. 62376242, Apr. 2014, doi: 10.1073/pnas.1316546111.

[97] Z. R. Barkley et al., "Quantifying methane emissions from natural gas production in north-eastern Pennsylvania," Atmos. Chem. Phys., vol. 17, no. 22, pp. 13941-13966, Nov. 2017, doi: 10.5194/acp17-13941-2017.

[98] J. G. Englander, A. R. Brandt, S. Conley, D. R. Lyon, and R. B. Jackson, "Aerial Interyear Comparison and Quantification of Methane Emissions Persistence in the Bakken Formation of North Dakota, USA," Environ. Sci. Technol., vol. 52, no. 15, pp. 8947-8953, Aug. 2018, doi: 10.1021/acs.est.8b01665.

[99] “Mobile Monitoring Challenge." https://methane.stanford.edu/ (accessed Mar. 08, 2021).

[100] A. P. Ravikumar et al., "Single-blind inter-comparison of methane detection technologies - results from the Stanford/EDF Mobile Monitoring Challenge," Elem Sci Anth, vol. 7, no. 1, p. 37, Sep. 2019, doi: $10.1525 /$ elementa.373.

[101]C. S. Bell, T. Vaughn, and D. Zimmerle, "Evaluation of next generation emission measurement technologies under repeatable test protocols," Elem Sci Anth, vol. 8, no. 1, p. 32, Jul. 2020, doi: 10.1525/elementa.426.

[102]L. Golston et al., "Natural Gas Fugitive Leak Detection Using an Unmanned Aerial Vehicle: Localization and Quantification of Emission Rate," Atmosphere, vol. 9, no. 9, p. 333, Aug. 2018, doi: 10.3390/atmos9090333.

[103]B. Travis, M. Dubey, and J. Sauer, "Neural networks to locate and quantify fugitive natural gas leaks for a MIR detection system," Atmospheric Environment: X, vol. 8, p. 100092, Dec. 2020, doi: 10.1016/j.aeaoa.2020.100092.

[104] T. A. Fox, T. E. Barchyn, D. Risk, A. P. Ravikumar, and C. H. Hugenholtz, "A review of close-range and screening technologies for mitigating fugitive methane emissions in upstream oil and gas," Environ. Res. Lett., vol. 14, no. 5, p. 053002, Apr. 2019, doi: 10.1088/1748-9326/ab0cc3.

[105] “J.W. Ruby Research Farm | Davis College of Agriculture, Natural Resources and Design | West Virginia University." https://www.davis.wvu.edu/about-davis-college/farms-and-forests/j-w-rubyresearch-farm (accessed Mar. 08, 2021).

[106] "MSEEL." http://mseel.org/ (accessed Jan. 02, 2020).

[107]D. R. Johnson, A. N. Covington, and N. N. Clark, "Design and Use of a Full Flow Sampling System (FFS) for the Quantification of Methane Emissions," JoVE, no. 112, p. 54179, Jun. 2016, doi: $10.3791 / 54179$.

[108]“LI-COR LI-7700.” LI-COR Biosciences. [Online]. Available: https://www.licor.com/env/support/LI-7700/manuals.html

[109]"LI-COR LI-7500.” LI-COR Biosciences. [Online]. Available: https://www.licor.com/env/support/LI-7500/home.html

[110]"LI-200R Pyranometer." LI-COR Biosciences. [Online]. Available: https://www.licor.com/env/products/light/pyranometer 
[111] Gill Instruments Limited, "WindMaster 3-Axis Ultrasonic Anemometer," Gill Instruments, 2015. http://www.gillinstruments.com/products/anemometer/windmaster.htm (accessed Jan. 02, 2020).

[112] “Omega iBTHx." [Online]. Available: https://in.omega.com/pptst/IBTX_IBTHX.html

[113] "BeagleBoard.org - black." https://beagleboard.org/black (accessed Mar. 08, 2021).

[114] "Python 2.7.0 Release," Python.org. https://www.python.org/download/releases/2.7/ (accessed Mar. 09, 2021).

[115] "Digi WR11 XT." https:/www.digi.com/products/networking/cellular-routers/industrial/digitransport-wr11-xt (accessed Mar. 08, 2021).

[116]"IoT SIM Card - Global Cellular M2M Connectivity | Hologram." https://www.hologram.io/products/iot-sim-card (accessed Mar. 08, 2021).

[117] "EddyPro 7 | Northward, eastward, and vertical separation." https://www.licor.com/env/support/EddyPro/topics/sensor-separation.html (accessed Mar. 08, 2021).

[118] "LG NeON R.” LG Electronics Inc. [Online]. Available: www.lg-solar.com

[119] “TriStar MPPT: Solar Charging System Controller.” Morning Star Corp. [Online]. Available: www.morningstarcorp.com

[120]"PVX-2120L Solar Battery." SunXtender. [Online]. Available: www.sunxtender.com

[121] "Ultra-Portable Greenhouse Gas Analyzer User Manual." Los Gatos Research, May 16, 2014. [Online]. Available: www.lgrinc.com

[122]"Los Gatos Research - Fast Methane/Ethane Analyzer (CH4, C2H6)." https://www.lgrinc.com/analyzers/overview.php?prodid=42 (accessed Mar. 09, 2021).

[123]"2003-2019 GM Mass Air Flow Sensor 15865791 | GMPartsDirect.com.” https://www.gmpartsdirect.com/oem-parts/gm-mass-air-flow-sensor-15865791 (accessed Mar. 09, 2021).

[124] "Laminar Flow Elements - Meriam," The Trusted Leader in Measurement \& Calibration Solutions. https://www.meriam.com/product-category/laminar-flow-element/ (accessed Mar. 09, 2021).

[125] "Eagle 2 Model." RKI Instruments. [Online]. Available: https://www.rkiinstruments.com/product/eagle-2/

[126]"MC-Gas Mass Flow Controllers," Alicat Scientific, Nov. 27, 2017. https://www.alicat.com/models/mc-gas-mass-flow-controllers/ (accessed Aug. 28, 2020).

[127] "Python Release Python 3.6.0," Python.org. https://www.python.org/downloads/release/python-360/ (accessed Jan. 02, 2020).

[128] “R2015a - MATLAB \& Simulink." https://www.mathworks.com/help/matlab/release-notesR2015a.html (accessed Jan. 02, 2020).

[129] “[Toolbox] - A rolling list of software/packages for flux-related data processing," FLUXNET, Oct. 10, 2017. https://fluxnet.org/2017/10/10/toolbox-a-rolling-list-of-softwarepackages-for-flux-relateddata-processing/ (accessed Mar. 08, 2021).

[130]"EddyPro 7 What is EddyPro?" https://www.licor.com/env/support/EddyPro/topics/introduction.html (accessed Mar. 08, 2021).

[131] "EddyPro 7 | Express default settings." https://www.licor.com/env/support/EddyPro/topics/expressdefaults.html (accessed Jan. 02, 2020).

[132] D. Vickers and L. Mahrt, "Quality Control and Flux Sampling Problems for Tower and Aircraft Data," JOURNAL OF ATMOSPHERIC AND OCEANIC TECHNOLOGY, vol. 14, p. 15, 1997.

[133] J. Moncrieff, R. Clement, J. Finnigan, and T. Meyers, "Averaging, Detrending, and Filtering of Eddy Covariance Time Series," in Handbook of Micrometeorology, vol. 29, X. Lee, W. Massman, and B. Law, Eds. Dordrecht: Kluwer Academic Publishers, 2005, pp. 7-31. doi: 10.1007/1-4020-2265-4_2.

[134]E. K. Webb, G. I. Pearman, and R. Leuning, "Correction of flux measurements for density effects due to heat and water vapour transfer," Q.J Royal Met. Soc., vol. 106, no. 447, pp. 85-100, Jan. 1980, doi: 10.1002/qj.49710644707.

[135] "EddyPro $7 \quad$ Flux quality flags for micrometeorological tests." https://www.licor.com/env/support/EddyPro/topics/flux-quality-flags.html (accessed Mar. 08, 2021). 
[136]T. Nakai and K. Shimoyama, "Ultrasonic anemometer angle of attack errors under turbulent conditions," Agricultural and Forest Meteorology, vol. 162-163, pp. 14-26, Sep. 2012, doi: 10.1016/j.agrformet.2012.04.004.

[137]R. N. Kacker, E. S. Lagergren, and J. J. Filliben, "Taguchi's orthogonal arrays are classical designs of experiments," J. RES. NATL. INST. STAN., vol. 96, no. 5, p. 577, Sep. 1991, doi: 10.6028/jres.096.034.

[138] "SciPy.org — SciPy.org." https://www.scipy.org/ (accessed Mar. 08, 2021).

[139]: : “OzFlux - Home ::” http://www.ozflux.org.au/ (accessed Mar. 08, 2021).

[140]OzFlux/OzFlux-footprint. OzFlux, 2020. Accessed: Mar. 08, 2021. [Online]. Available: https://github.com/OzFlux/OzFlux-footprint

[141] "Probability Distributions for Measurement Uncertainty," isobudgets, Oct. 28, 2015. https://www.isobudgets.com/probability-distributions-for-measurement-uncertainty/ (accessed Mar. 08, 2021).

[142] "How to Calculate Resolution Uncertainty," isobudgets, Apr. 29, 2016. https://www.isobudgets.com/calculate-resolution-uncertainty/ (accessed Mar. 08, 2021).

[143] "Estimating Measurement Uncertainty." https://www.sml-inc.com/uncertainy.htm (accessed Mar. 08, 2021).

[144]"EddyPro $\quad 7 \quad$ Random uncertainty estimation." https://www.licor.com/env/support/EddyPro/topics/random-uncertaintyestimation.html?Highlight=Finkelstein\%20and\%20Sims (accessed Mar. 08, 2021).

[145] S. Yamulki, S. C. Jarvis, and P. Owen, "Methane Emission and Uptake from Soils as Influenced by Excreta Deposition from Grazing Animals," J. environ. qual., vol. 28, no. 2, pp. 676-682, Mar. 1999, doi: $10.2134 /$ jeq1999.00472425002800020036x.

[146] "Environmental Growth Chambers." http://www.egc.com/useful_info_lighting.php (accessed Mar. 09, 2021).

[147] Ü. Rannik, O. Peltola, and I. Mammarella, "Random uncertainties of flux measurements by the eddy covariance technique," Atmos. Meas. Tech., vol. 9, no. 10, pp. 5163-5181, Oct. 2016, doi: 10.5194/amt-9-5163-2016.

[148] "scikit-learn: machine learning in Python — scikit-learn 0.24.1 documentation." https://scikitlearn.org/stable/ (accessed Mar. 08, 2021).

[149] “Cloud Computing Services | Microsoft Azure." https://azure.microsoft.com/en-us/ (accessed Mar. 08, 2021).

[150]"SAS: Analytics, Artificial Intelligence and Data Management." https://www.sas.com/en_us/home.html (accessed Mar. 08, 2021).

[151]T. Hastie, R. Tibshirani, and J. Friedman, The Elements of Statistical Learning: Data Mining, Inference, and Prediction, Second. Springer, 2008.

[152] S. Wager, T. Hastie, and B. Efron, "Confidence Intervals for Random Forests: The Jackknife and the Infinitesimal Jackknife," p. 27.

[153] K. Polimis, A. Rokem, and B. Hazelton, "Confidence Intervals for Random Forests in Python," JOSS, vol. 2, no. 19, p. 124, Nov. 2017, doi: 10.21105/joss.00124.

[154] J. Leonel, jorgesleonel/Multilayer-Perceptron. 2021. Accessed: Mar. 08, 2021. [Online]. Available: https://github.com/jorgesleonel/Multilayer-Perceptron

[155] "Perceptrons \& Multi-Layer Perceptrons: the Artificial Neuron - MissingLink," MissingLink.ai. https://missinglink.ai/guides/neural-network-concepts/perceptrons-and-multi-layer-perceptrons-theartificial-neuron-at-the-core-of-deep-learning/ (accessed Mar. 08, 2021).

[156]"1.17. Neural network models (supervised) — scikit-learn 0.24.1 documentation." https://scikitlearn.org/stable/modules/neural_networks_supervised.html (accessed Mar. 08, 2021).

[157] "sklearn.ensemble.RandomForestRegressor - scikit-learn 0.24.1 documentation." https://scikitlearn.org/stable/modules/generated/sklearn.ensemble.RandomForestRegressor.html (accessed Mar. 08, 2021).

[158] J. Bergstra and Y. Bengio, "Random Search for Hyper-Parameter Optimization," p. 25. 
[159]"sklearn.model_selection.RandomizedSearchCV $\quad$ - scikit-learn 0.24 .1 documentation." https://scikit-

learn.org/stable/modules/generated/sklearn.model_selection.RandomizedSearchCV.html (accessed Mar. 08, 2021).

[160] "model selection - How to choose the number of hidden layers and nodes in a feedforward neural network?," Cross Validated. https://stats.stackexchange.com/questions/181/how-to-choose-thenumber-of-hidden-layers-and-nodes-in-a-feedforward-neural-netw (accessed Mar. 08, 2021).

[161] "sklearn.neural_network.MLPRegressor — scikit-learn 0.24.1 documentation." https://scikitlearn.org/stable/modules/generated/sklearn.neural_network.MLPRegressor.html\#sklearn.neural_net work.MLPRegressor (accessed Mar. 08, 2021). 


\section{Appendix A}

Table A1. Highly Correlated Features (HCF)

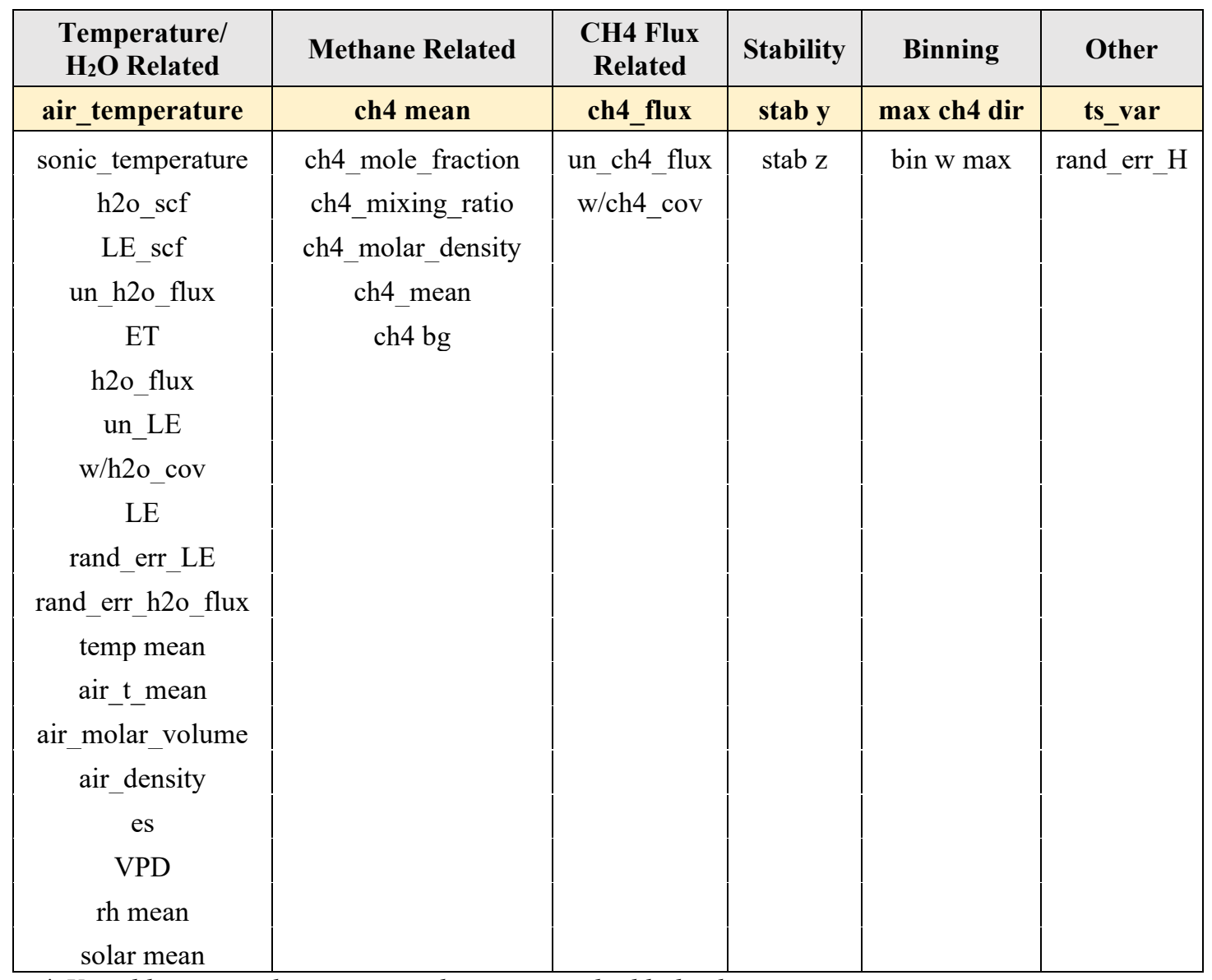

* Variables retained to represent the group are highlighted. 